\title{
Radical Addition-Triggered Remote Migratory Isomerization of Unactivated Alkenes to Difluoromethylene-Containing Alkenes Enabled by Bimetallic Catalysis
}

\author{
Qiao Zhang, ${ }^{\dagger}$ Simin Wang, ${ }^{\dagger}$ Qian Zhang, ${ }^{\dagger}$ Tao Xiong, ${ }^{* \dagger}$ and Qian Zhang* ${ }^{*}$ \\ E-mail: xiongt626@nenu.edu.cn; zhangq651@nenu.edu.cn
}

†Key Laboratory of Functional Organic Molecule Design \& Synthesis of Jilin Province, Department of Chemistry, Northeast Normal University, 5268 Renmin Rd, Changchun, Jilin 130024, China

${ }^{\ddagger}$ State Key Laboratory of Organometallic Chemistry, Shanghai Institute of Organic Chemistry, Chinese Academy of Sciences 345 Lingling Lu, Shanghai 200032, China

\section{Table of Contents}

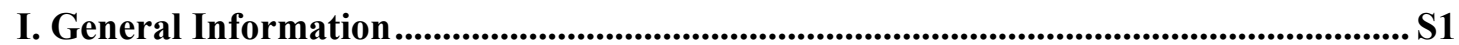

II. General procedure for the synthesis of difluoromethylene-containing alkenes enabled

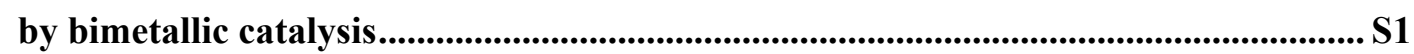

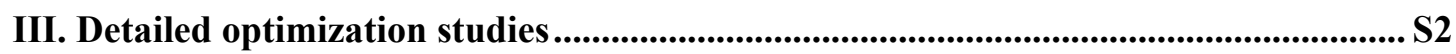

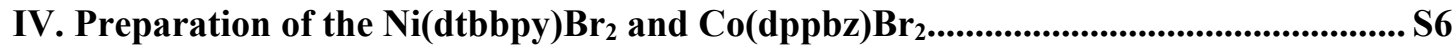

V. Preparation the substrate of the alkenes ................................................................................. S6

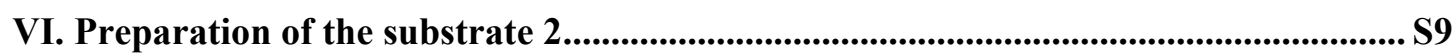

VII. Unsuccessful examples ........................................................................................................... S10

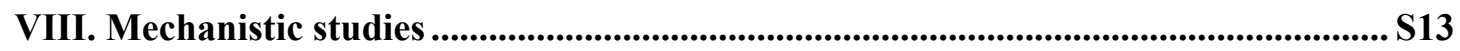

IX. Functionalization and application ..................................................................................... S29

X. References ...................................................................................................................................... S34

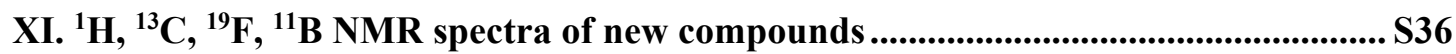




\section{General Information}

All reactions were carried out under nitrogen atmosphere in a glovebox. Reagents were purchased from commercial sources and used without further purification, unless otherwise noted. All of the solvents were anhydrous according distillation. The reactions were monitored with the aid of thinlayer chromatography (TLC) on $0.25 \mathrm{~mm}$ precoated silica gel plates. Visualization was carried out with UV light and aqueous potassium permanganate stain. Melting points were measured on Büchi B-540 apparatus. ${ }^{1} \mathbf{H}$ NMR spectra were recorded at $25^{\circ} \mathrm{C}$ on a Bruker 600 or 500 , Varian $500 \mathrm{MHz}$, ${ }^{13} \mathrm{C}$ NMR spectra were recorded at $25^{\circ} \mathrm{C}$ on a Bruker 151 , Varian $126 \mathrm{MHz}$, respectively in $\mathrm{CDCl}_{3}$ by using TMS as internal standard. ${ }^{19} \mathbf{F}$ NMR spectra were recorded at $25{ }^{\circ} \mathrm{C}$ on a Bruker 565 or $471 \mathrm{MHz}$. Chemical shifts $(\delta)$ are given in ppm relative to the residual solvent signals (chloroform, 7.26 ppm for ${ }^{1} \mathrm{H}-\mathrm{NMR}$ and $77.00 \mathrm{ppm}$ for ${ }^{13} \mathrm{C}$-NMR). ${ }^{11} \mathbf{B}$ NMR spectra were recorded at $25{ }^{\circ} \mathrm{C}$ on a Bruker 193 MHz. Coupling constants (J) are given in Hertz (Hz). Letters m, s, d, t and q stand for multiplet, singlet, doublet, triplet and quartet, respectively. High resolution mass spectra (HRMS) were recorded on Bruck microtof. High-pressure liquid chromatography (HPLC) was performed on Agilent 1260 Series chromatographs using a chiral column $(25 \mathrm{~cm})$ as noted for each compound. Enantiomeric excess was determined by HPLC analysis employing Darcel Chiracel OD and IC column. Optical rotation was measured on a Perkin-Elmer 341 polarimeter using a sodium lamp $(589 \mathrm{~nm})$ as the light source over a path length of $10 \mathrm{~cm}$. UV-Visible Absorption Spectra was measured on an UV-Visible Spectrophotometer (Cary 60). The Catalyst of $\mathrm{Ni}(\mathrm{dtbbpy}) \mathrm{Br}_{2}$ and $\mathrm{Co}(\mathrm{dppbz}) \mathrm{Br}_{2}$ were prepared according to the literature.

\section{General procedure for the synthesis of difluoromethylene-containing alkenes enabled by bimetallic catalysis}

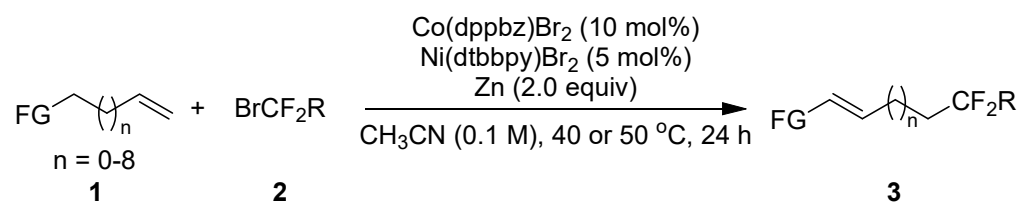

Into a nitrogen-filled glove box, a vial $(10.0 \mathrm{~mL})$ was charged with $\mathrm{Co}(\mathrm{dppbz}) \mathrm{Br}_{2}(13.3 \mathrm{mg}, 10 \mathrm{~mol} \%)$, $\mathrm{Zn}$ powder $(26.0 \mathrm{mg}, 0.4 \mathrm{mmol})$ and anhydrous $\mathrm{CH}_{3} \mathrm{CN}(2.0 \mathrm{~mL})$. The reaction mixture was stirred at room temperature for $5 \mathrm{~min}$, then $\mathrm{Ni}(\mathrm{dtbbpy}) \mathrm{Br}_{2}(4.9 \mathrm{mg}, 5 \mathrm{~mol} \%), \mathbf{1}(0.2 \mathrm{mmol})$ and $\mathbf{2}(0.4 \mathrm{mmol})$ were added. The vial was removed from the glovebox and stirred at 40 or $50^{\circ} \mathrm{C}$ for $24 \mathrm{~h}$. After the reaction finished that monitored by TLC, the reaction mixture was cooled to room temperature and quenched by water. The mixture was extracted with $\mathrm{Et}_{2} \mathrm{O}(3 \times 5.0 \mathrm{~mL})$. The combined organic phases were dried over anhydrous $\mathrm{Na}_{2} \mathrm{SO}_{4}$ and the solvent was evaporated under vacuum. The residue was purified by flash column chromatography (petroleum ether / diethyl ether $=100 / 1$ ) to give the corresponding product 3 . 
III. Detailed Optimization Studies

Table S1 Ni catalyst screening ${ }^{a}$

(II

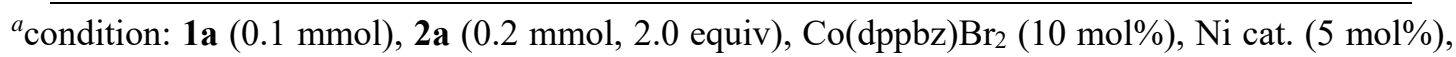
$\mathrm{Zn}\left(0.2 \mathrm{mmol}, 2.0\right.$ equiv), $\mathrm{CH}_{3} \mathrm{CN}(1.0 \mathrm{~mL}, 0.1 \mathrm{M}), 40{ }^{\circ} \mathrm{C}, 24 \mathrm{~h}$. The yields determined by crude NMR using dibromomethane $\left(\mathrm{CH}_{2} \mathrm{Br}_{2}\right)$ as an internal standard. 
Table S2 Co catalyst screening ${ }^{a}$

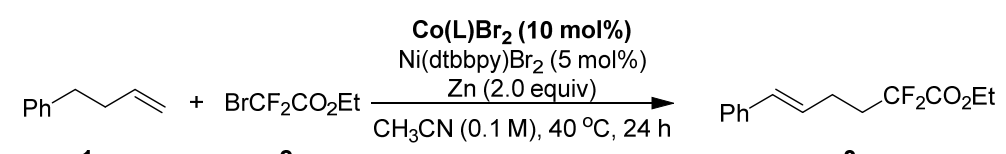

2a

$3 a$<smiles>CCOC(=O)[C-]CC(Br)CCc1ccccc1</smiles>

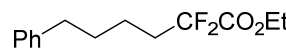<smiles>CCOC(=O)[C-](C/C=C/Cc1ccccc1)CCOC</smiles>

$4 a$

$5 a$

$6 a$

\begin{tabular}{cccccc}
\hline entry & Co cat. & $\begin{array}{c}\text { 3a } \\
\text { (yield \%) }\end{array}$ & $\begin{array}{c}\text { 4a } \\
\text { (yield \%) }\end{array}$ & $\begin{array}{c}\text { 5a } \\
\text { (yield \%) }\end{array}$ & $\begin{array}{c}\text { 6a } \\
\text { (yield \%) }\end{array}$ \\
\hline 1 & $\begin{array}{c}\mathrm{Co}\left(\mathrm{PPh}_{3}\right)_{2} \mathrm{Br}_{2} \\
\mathrm{Co}(\mathrm{xantphos}) \mathrm{Br}_{2}\end{array}$ & 0 & 0 & 0 & 0 \\
2 & $\mathrm{Co}(\mathrm{dpephos}) \mathrm{Br}_{2}$ & 0 & 0 & 0 & 0 \\
$\mathrm{Co}(\mathrm{binap}) \mathrm{Br}_{2}$ & 0 & 0 & 0 & 0 \\
$\mathrm{Co}(\mathrm{dppp}) \mathrm{Br}_{2}$ & 0 & 0 & 19 & 8 \\
3 & & &
\end{tabular}

${ }^{a}$ condition: 1a ( $\left.0.1 \mathrm{mmol}\right), \mathbf{2 a}\left(0.2 \mathrm{mmol}, 2.0\right.$ equiv), $\mathrm{Co}(\mathrm{L}) \mathrm{Br}_{2}(10 \mathrm{~mol} \%), \mathrm{Ni}(\mathrm{dtbbpy}) \mathrm{Br}_{2}(5 \mathrm{~mol} \%)$, $\mathrm{Zn}\left(0.2 \mathrm{mmol}, 2.0\right.$ equiv), $\mathrm{CH}_{3} \mathrm{CN}(1.0 \mathrm{~mL}, 0.1 \mathrm{M}), 40{ }^{\circ} \mathrm{C}, 24 \mathrm{~h}$. The yields determined by crude NMR using dibromomethane $\left(\mathrm{CH}_{2} \mathrm{Br}_{2}\right)$ as an internal standard.

Table S3 The ratio of the catalyst ${ }^{a}$

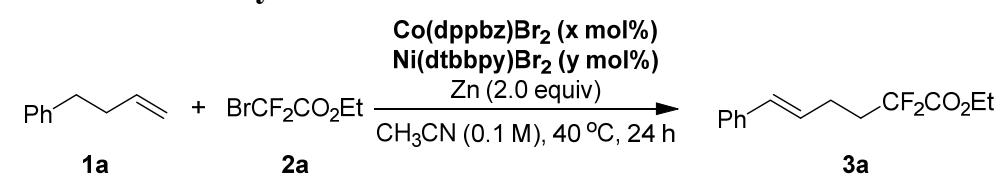

$1 \mathrm{a}$

2a

$3 a$<smiles>CCOC(=O)CC(Br)CCc1ccccc1</smiles>

$\mathrm{Ph} \sim \mathrm{CF}_{2} \mathrm{CO}_{2} \mathrm{Et}$

$\mathrm{Ph} ح \mathrm{~mm}_{2}-\mathrm{CF}_{2} \mathrm{CO}_{2} \mathrm{Et}$

$4 a$

$5 a$

\begin{tabular}{ccccccc}
\hline entry & $\begin{array}{c}\text { Co cat. } \\
(\mathbf{x} \%)\end{array}$ & $\begin{array}{c}\text { Ni cat. } \\
(\mathbf{y} \%)\end{array}$ & $\begin{array}{c}\text { 3a } \\
(\mathbf{y i e l d} \%)\end{array}$ & $\begin{array}{c}\text { 4a } \\
(\mathbf{y i e l d} \%)\end{array}$ & $\begin{array}{c}\mathbf{5 a} \\
\text { (yield \%) }\end{array}$ & $\begin{array}{c}\text { 6a } \\
\text { (yield \%) }\end{array}$ \\
\hline 1 & 10 & 3 & 62 & 0 & 15 & 4 \\
2 & 10 & 5 & 78 & 0 & 12 & 4 \\
3 & 15 & 7.5 & 76 & 0 & 13 & 5 \\
4 & 15 & 10 & 77 & 0 & 12 & 4 \\
5 & 20 & 10 & 74 & 0 & 11 & 6 \\
6 & 10 & 10 & 66 & 0 & 13 & 5 \\
7 & 10 & 15 & 37 & 0 & 13 & 15 \\
\hline
\end{tabular}

${ }^{a}$ condition: 1a $(0.1 \mathrm{mmol}), \mathbf{2 a}\left(0.2 \mathrm{mmol}, 2.0\right.$ equiv), $\mathrm{Co}(\mathrm{dppbz}) \mathrm{Br}_{2}$ (x mol\%), Ni(dtbbpy)Br $\mathrm{Br}_{2}(\mathrm{y}$ mol\%), $\mathrm{Zn}\left(0.2 \mathrm{mmol}, 2.0\right.$ equiv), $\mathrm{CH}_{3} \mathrm{CN}(1.0 \mathrm{~mL}, 0.1 \mathrm{M}), 40{ }^{\circ} \mathrm{C}, 24 \mathrm{~h}$. The yields determined by crude NMR using dibromomethane $\left(\mathrm{CH}_{2} \mathrm{Br}_{2}\right)$ as an internal standard. 
Table S4 Solvent screening ${ }^{a}$

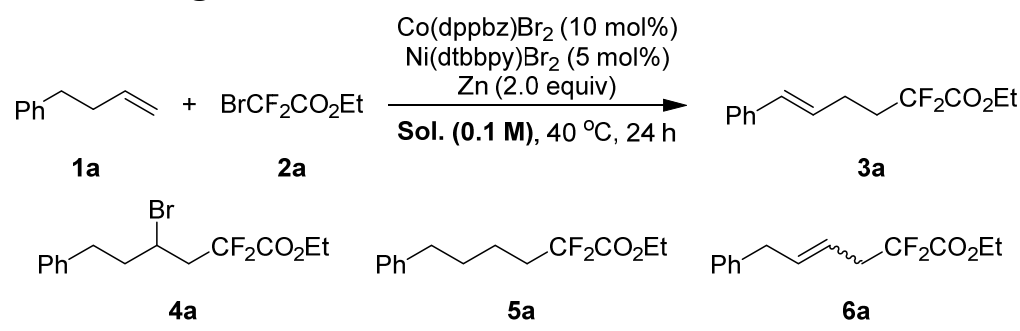

\begin{tabular}{cccccc}
\hline entry & Sol. & $\begin{array}{c}\text { 3a } \\
\text { (yield \%) }\end{array}$ & $\begin{array}{c}\text { 4a } \\
\text { (yield \%) }\end{array}$ & $\begin{array}{c}\text { 5a } \\
\text { (yield \%) }\end{array}$ & $\begin{array}{c}\text { 6a } \\
\text { (yield \%) }\end{array}$ \\
\hline 1 & THF & 0 & 0 & 38 & 10 \\
2 & Dioxane & 0 & 42 & 16 & 8 \\
3 & DCM & 0 & 86 & trace & 0 \\
4 & Tol. & 0 & 24 & 19 & 7 \\
5 & $\mathrm{EA}$ & 0 & 0 & 37 & 9 \\
6 & $\mathrm{CH}_{3} \mathrm{CN}$ & 78 & 0 & 12 & 4 \\
7 & $\mathrm{EtCN}$ & 67 & 0 & 19 & 4 \\
8 & ${ }^{i} \mathrm{PrCN}$ & 64 & 0 & 19 & 7 \\
9 & ${ }^{t} \mathrm{BuCN}$ & 28 & 16 & 15 & 3 \\
\hline
\end{tabular}

${ }^{a}$ condition: 1a (0.1 mmol), 2a ( $0.2 \mathrm{mmol}, 2.0$ equiv), $\mathrm{Co}(\mathrm{dppbz}) \mathrm{Br}_{2}(10 \mathrm{~mol} \%), \mathrm{Ni}(\mathrm{dtbbpy}) \mathrm{Br}_{2}(5$ mol\%), Zn (0.2 mmol, 2.0 equiv), Sol. (1.0 mL, $0.1 \mathrm{M}), 40{ }^{\circ} \mathrm{C}, 24 \mathrm{~h}$. The yields determined by crude NMR using dibromomethane $\left(\mathrm{CH}_{2} \mathrm{Br}_{2}\right)$ as an internal standard.

Table S5 Temperature screening ${ }^{a}$

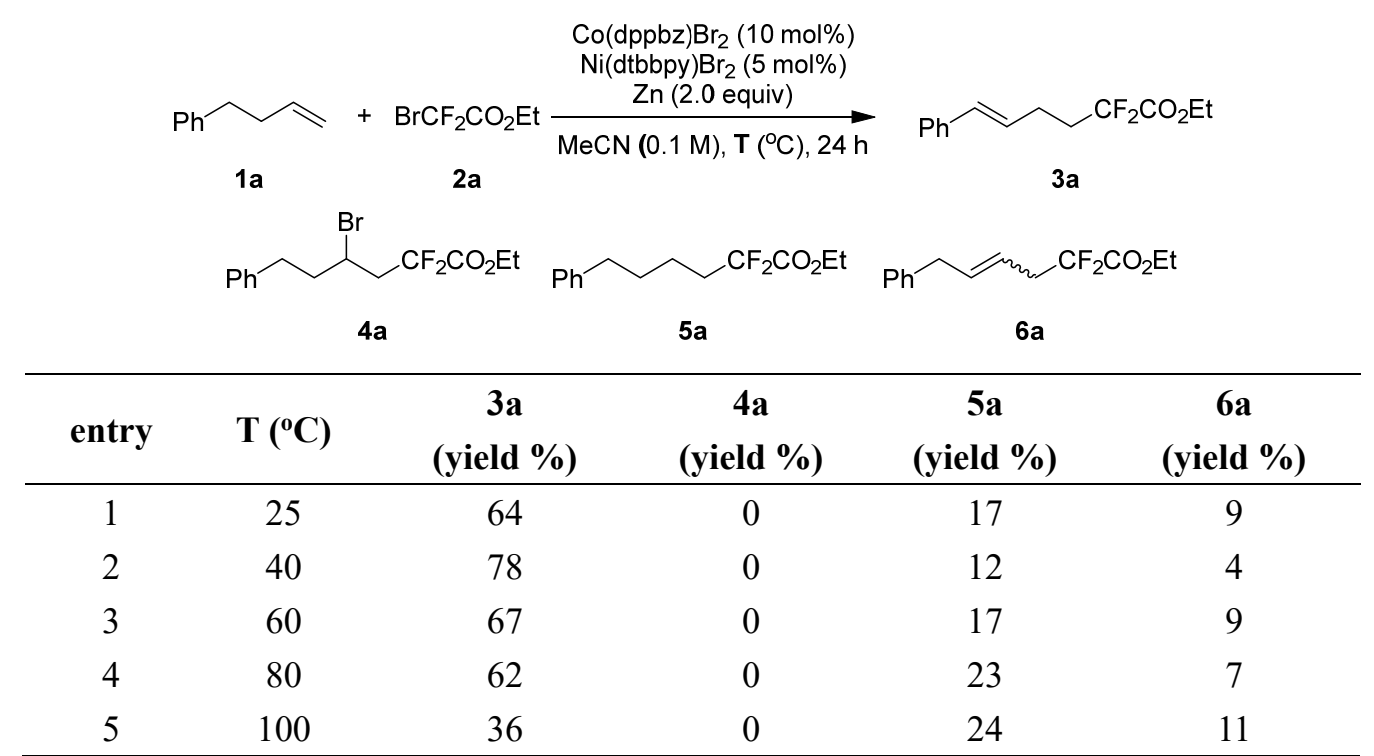

${ }^{a}$ condition: 1a $(0.1 \mathrm{mmol}), \mathbf{2 a}\left(0.2 \mathrm{mmol}, 2.0\right.$ equiv), $\mathrm{Co}(\mathrm{dppbz}) \mathrm{Br}_{2}(10 \mathrm{~mol} \%), \mathrm{Ni}(\mathrm{dtbbpy}) \mathrm{Br}_{2}(5$ mol\%), $\mathrm{Zn}$ (0.2 mmol, 2.0 equiv), $\mathrm{CH}_{3} \mathrm{CN}(1.0 \mathrm{~mL}, 0.1 \mathrm{M}), \mathrm{T}\left({ }^{\circ} \mathrm{C}\right), 24 \mathrm{~h}$. The yields determined by crude NMR using dibromomethane $\left(\mathrm{CH}_{2} \mathrm{Br}_{2}\right)$ as an internal standard. 
Table S6 Additive screening ${ }^{a}$

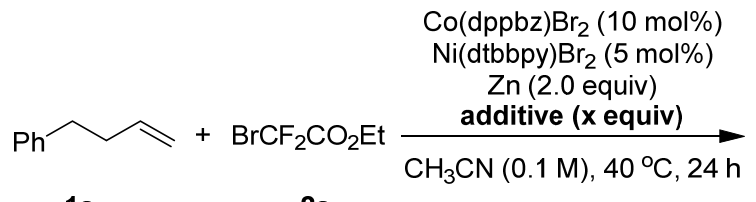

$1 a$<smiles>CCOC(=O)[C-]CC(Br)CCc1ccccc1</smiles>

$4 a$

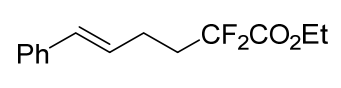

$3 a$

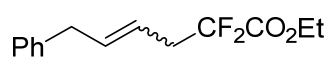

$6 a$

\begin{tabular}{cccccc}
\hline entry & $\begin{array}{c}\text { Additive } \\
(\mathbf{e q})\end{array}$ & $\begin{array}{c}\text { 3a } \\
\text { (yield \%) }\end{array}$ & $\begin{array}{c}\text { 4a } \\
\text { (yield \%) }\end{array}$ & $\begin{array}{c}\text { 5a } \\
\text { (yield \%) }\end{array}$ & $\begin{array}{c}\text { 6a } \\
\text { (yield \%) }\end{array}$ \\
\hline 1 & $\mathrm{NaI}(0.5)$ & 41 & 0 & 32 & 15 \\
2 & $\mathrm{MgCl}_{2}(0.5)$ & 22 & 21 & 22 & 29 \\
3 & $\mathrm{LiCl}(0.5)$ & 38 & 0 & 30 & 26 \\
4 & $\mathrm{ZnI}_{2}(0.1)$ & 70 & 0 & 20 & 5 \\
\hline
\end{tabular}

${ }^{a}$ condition: 1a (0.1 mmol), 2a (0.2 mmol, 2.0 equiv), Co(dppbz)Br $\mathrm{B}_{2}(10 \mathrm{~mol} \%), \mathrm{Ni}(\mathrm{dtbbpy}) \mathrm{Br}_{2}(5$ mol\%), $\mathrm{Zn}\left(0.2 \mathrm{mmol}, 2.0\right.$ equiv), additive (x equiv), $\mathrm{CH}_{3} \mathrm{CN}(1.0 \mathrm{~mL}, 0.1 \mathrm{M}), 40{ }^{\circ} \mathrm{C}, 24 \mathrm{~h}$. The yields determined by crude NMR using dibromomethane $\left(\mathrm{CH}_{2} \mathrm{Br}_{2}\right)$ as an internal standard. 


\section{Preparation of the $\mathrm{Ni}(\mathrm{dtbbpy}) \mathrm{Br}_{2}$ and $\mathrm{Co}(\mathrm{dppbz}) \mathrm{Br}_{2}$}

Preparation of $\left[\mathbf{N i}(\mathbf{d t b b p y}) \mathrm{Br}_{2}\right]^{1}$ : A $250 \mathrm{~mL}$, round bottom flask was equipped with a tefloncoated magnetic stir bar, $\mathrm{NiBr}_{2} 3 \mathrm{H}_{2} \mathrm{O}(1.36 \mathrm{~g}, 5.0 \mathrm{mmol}$ ), 4,4'-di-tert-butyl-2,2'-bipyridine (dtbbpy, $1.48 \mathrm{~g}, 5.5 \mathrm{mmol}, 1.1$ equiv), anhydrous ethanol $(25.0 \mathrm{~mL})$ and a condenser with a rubber septum under nitrogen atmosphere. The reaction was heated at $90{ }^{\circ} \mathrm{C}$ for $12 \mathrm{~h}$. The reaction was then cooled to room temperature and the solvent was reduced in vacuo to $\sim 10 \%$ of its original volume. A 1:4 mixture of THF : $\mathrm{Et}_{2} \mathrm{O}(150 \mathrm{~mL})$ was added to the residue and the mixture was stirred for $3 \mathrm{~h}$ under nitrogen. The mixture was then filtered, washed with hexane $(2 \times 50.0 \mathrm{~mL})$, and the collected light green solid was dried under vacuum for $12 \mathrm{~h}$ and stored in the glove box ( $2.2 \mathrm{~g}, 90 \%$ yield).

Preparation of $\left[\mathbf{C o}(\mathbf{d p p b z}) \mathrm{Br}_{2}\right]^{2}$ : Compound $\mathrm{Co}(\mathrm{dppbz}) \mathrm{Br}_{2}$ was prepared according to a modified procedure reported in literature. In the glovebox, to a solution of anhydrous $\mathrm{CoBr}_{2}(1.09 \mathrm{~g}, 5.0 \mathrm{mmol})$ in THF $(25.0 \mathrm{~mL}$ ) was added a solution of 1,2-Bis(diphenylphosphino)benzene (dppbz, $2.46 \mathrm{~g}, 5.5$ mmol, 1.1 equiv) in THF $(30.0 \mathrm{~mL})$ in a $100 \mathrm{~mL}$ round bottom flask. The mixture changed color from blue-green to dark brown and the resulting solution was stirred for $12 \mathrm{~h}$ at room temperature. After evaporation of all volatiles in vacuo, anhydrous $\mathrm{Et}_{2} \mathrm{O}(50.0 \mathrm{~mL})$ was added and stirred for 10 min under nitrogen atmosphere, subsequently filtrated and washed with hexane $(2 \times 50.0 \mathrm{~mL})$. The remaining volatiles were removed in vacuo to yield $\mathrm{Co}(\mathrm{dppbz}) \mathrm{Br}_{2}$ as a dark brown powder and stored in the glove box $(3.03 \mathrm{~g}, 91 \%$ yield $)$.

\section{Preparation the substrate of the alkenes}

The substrate of the alkenes were prepared according to the procedures reported in the literature.

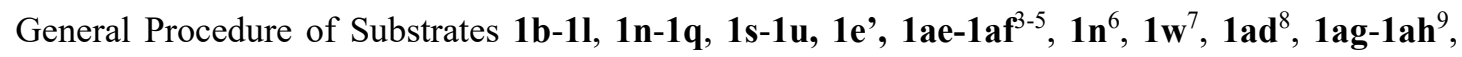
$\mathbf{1} \mathbf{a i}^{10}, \mathbf{1} \mathbf{j}^{11}, \mathbf{1} \mathbf{a k}^{12}$.

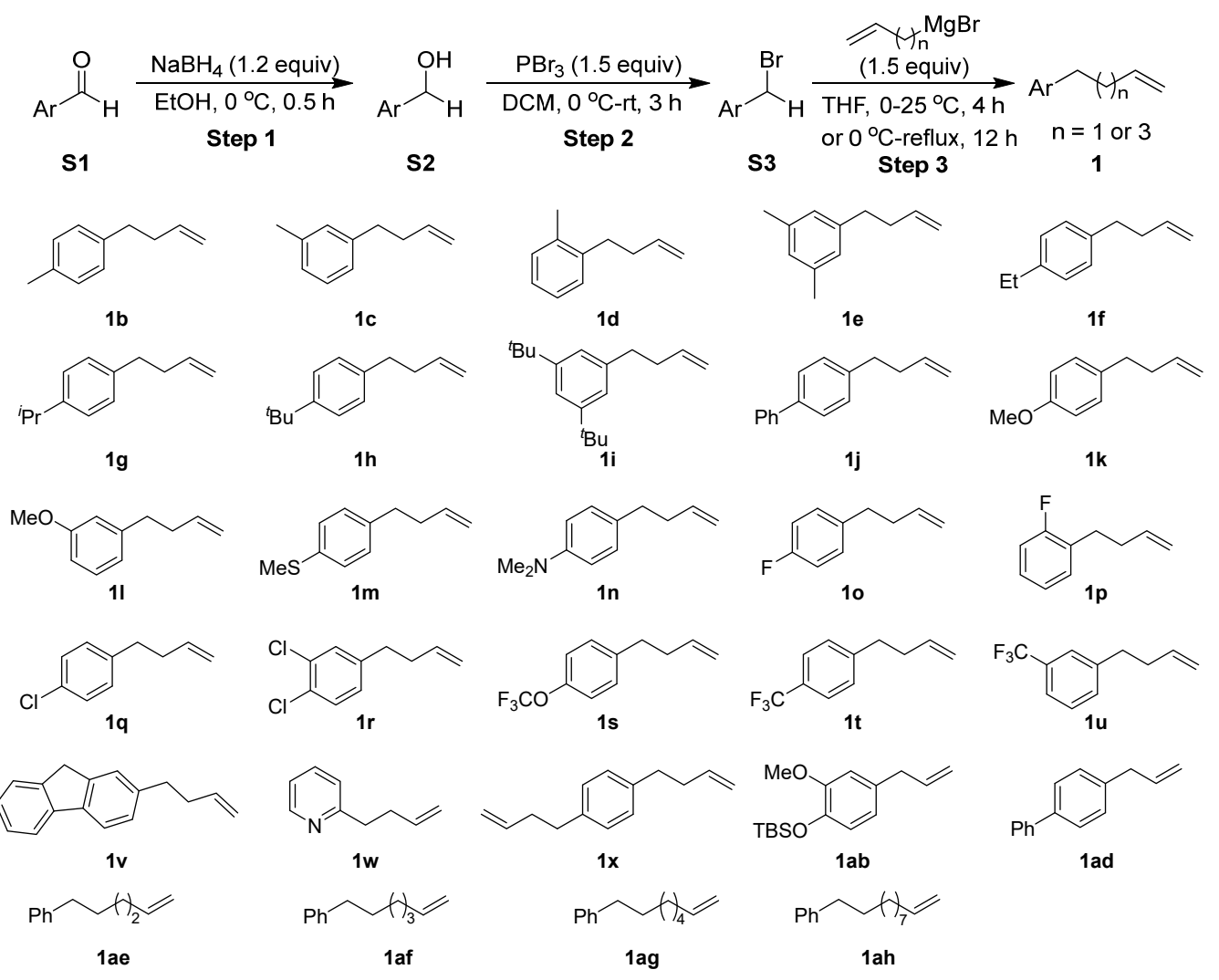




\section{General procedure A:}

Step 13: To a solution of sodium borohydride $\left(\mathrm{NaBH}_{4}, 12.0 \mathrm{mmol}, 1.2\right.$ equiv) in anhydrous EtOH $(15.0 \mathrm{~mL})$ was added a solution of $\mathbf{S 1}\left(10.0 \mathrm{mmol}, 1.0\right.$ equiv) in anhydrous $\mathrm{EtOH}(10.0 \mathrm{~mL})$ at $0{ }^{\circ} \mathrm{C}$, and the mixture was stirred for $30 \mathrm{~min}$ at $0{ }^{\circ} \mathrm{C}$. After consumption of all starting material (as monitored by TLC), water was added, the resultant mixture was extracted with DCM ( $3 \times 30.0 \mathrm{~mL})$. The combined organic layer was washed with saturated aqueous $\mathrm{NaCl}(30.0 \mathrm{~mL})$, and dried over anhydrous $\mathrm{Na}_{2} \mathrm{SO}_{4}$, filtered off, the solvent was removed in vacuo. The residue was purified by column chromatography on silica gel using petroleum ether / ethyl acetate (5 / 1) as eluent, to give the compound $\mathbf{S 2}$.

Step 24: To a solution of $\mathbf{S 2}\left(8.0 \mathrm{mmol}, 1.0\right.$ equiv) in DCM $(15.0 \mathrm{~mL})$ at $0{ }^{\circ} \mathrm{C}$ was slowly added phosphorus tribromide $\left(\mathrm{PBr}_{3}, 12.0 \mathrm{mmol}, 1.5\right.$ equiv) under nitrogen atmosphere. The reaction mixture was allowed naturally to come to room temperature while stirring for $3 \mathrm{~h}$. The reaction was quenched with water $(30.0 \mathrm{~mL})$ and the aqueous phase extracted with DCM $(3 \times 20.0 \mathrm{~mL})$. The combined organic layers were washed with saturated aqueous $\mathrm{NaCl}(30.0 \mathrm{~mL})$ and dried over anhydrous $\mathrm{Na}_{2} \mathrm{SO}_{4}$, filtered off and the solvent was removed in vacuo, afford the crude product $\mathbf{S 3}$. The crude product $\mathbf{S 3}$ was used directly for the next step.

Step $3^{5}$ : The product $\mathbf{S 3}(5.0 \mathrm{mmol})$ and $15.0 \mathrm{~mL}$ anhydrous THF were added to a flame dried 100 $\mathrm{mL}$ round-bottom flask. Alkylmagnesium bromide $(7.5 \mathrm{mmol}, 1 \mathrm{M}$ in diethylether) was added dropwise at $0{ }^{\circ} \mathrm{C}$. The reaction was stirred for $4 \mathrm{~h}$ at room temperature or reflux for $12 \mathrm{~h}$ under $\mathrm{N}_{2}$. After consumption of all starting material (as monitored by TLC), then quenched with saturated aqueous $\mathrm{NH}_{4} \mathrm{Cl}$. The aqueous layer was extracted with DCM $(3 \times 20.0 \mathrm{~mL})$ and the combined organic layers were dried over $\mathrm{Na}_{2} \mathrm{SO}_{4}$, filtered off and concentrated under reduced pressure. The crude product was purified by flash chromatography (eluent: petroleum ether), affording the desired product 1.

\section{General procedure $\mathbf{B}^{6}$ :}<smiles>C=CCCc1ccc(N(C)C)cc1</smiles>

To a dispersion of p-bromo-N,N-dimethylaniline $(2.0 \mathrm{~g}, 10.0 \mathrm{mmol})$ and $\mathrm{Pd}(\mathrm{dppf}) \mathrm{Cl}_{2}(73.1 \mathrm{mg}, 0.1$ mmol) in dried THF $(30.0 \mathrm{~mL})$ under nitrogen atmosphere, a dried THF solution $(50.0 \mathrm{~mL})$ of 4butenylmagnesium bromide ( $22.0 \mathrm{mmol}, 2.2$ equiv) was added dropwise at room temperature. The solution was then refluxed for $6 \mathrm{~h}$. After removing the solvent under a vacuum, DCM was added and the mixture was washed with a dilute aqueous $\mathrm{NH}_{4} \mathrm{Cl}$ solution, dried over $\mathrm{Na}_{2} \mathrm{SO}_{4}$, filtered off and concentrated under reduced pressure. The product was purified by column chromatography (petroleum ether / ethyl acetate (30 / 1)) to give the product ( $1.5 \mathrm{~g}, 86 \%$ yield). 


\section{General procedure $\mathbf{C}^{7}$ :}

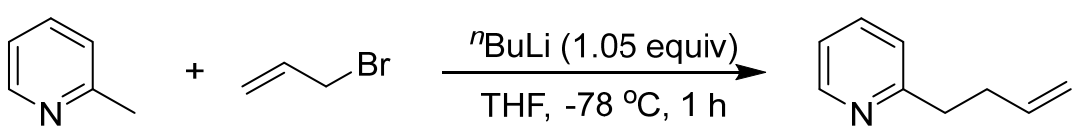

A solution of $n-\mathrm{BuLi}$ (1.05 equiv) was added dropwise to a solution of 2-methylpyridine (10.0 mmol) in THF $(30.0 \mathrm{~mL})$ at $-78{ }^{\circ} \mathrm{C}$ under nitrogen atmosphere and the reaction mixture was allowed to stir at this temperature for $15 \mathrm{~min}$. The solution was then brought to $0{ }^{\circ} \mathrm{C}$ for $15 \mathrm{~min}$, after which allyl bromide ( 1.05 equiv) was added at $-78{ }^{\circ} \mathrm{C}$. The resultant mixture was stirred for $30 \mathrm{~min}$ at $-78{ }^{\circ} \mathrm{C}$, after the reaction finished that detected by TLC, quenched with $\mathrm{MeOH}(2.0 \mathrm{~mL})$ and stirred for 30 min, brought to room temperature, and diluted with $\mathrm{H}_{2} \mathrm{O}(50.0 \mathrm{~mL})$ and ethyl acetate $(50.0 \mathrm{~mL})$. The reaction mixture was extracted with ethyl acetate $(3 \times 20.0 \mathrm{~mL})$, and combined organics were washed with brine, dried over anhydrous $\mathrm{Na}_{2} \mathrm{SO}_{4}$, filtered off and concentrated under reduced pressure, the residue was purified by column chromatography on silica gel (petroleum ether / ethyl acetate $(50 / 1))$ as eluent to afford the product $(0.9 \mathrm{~g}, 68 \%$ yield $)$.

\section{General procedure $\mathbf{D}^{8}$ :}

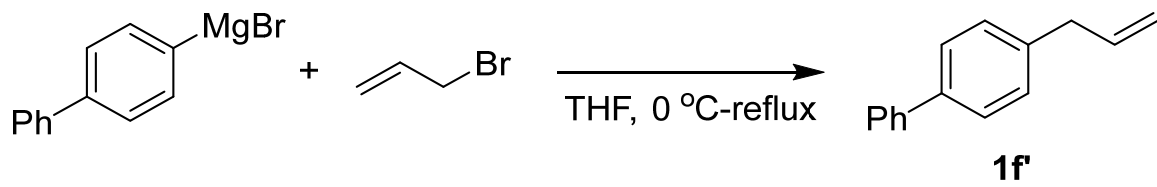

To a solution of Arylmagnesium bromide $(1.85 \mathrm{~g}, 10.0 \mathrm{mmol})$ in dry THF $(30.0 \mathrm{~mL})$ was added allyl bromide $\left(15.0 \mathrm{mmol}, 1.0 \mathrm{M}\right.$ solution in $\mathrm{Et}_{2} \mathrm{O}, 1.5$ equiv) at $0{ }^{\circ} \mathrm{C}$ under nitrogen atmosphere dropwise. After being stirred at reflux for $12 \mathrm{~h}$, the reaction mixture was quenched with saturated aqueous $\mathrm{NH}_{4} \mathrm{Cl}$, extracted with DCM $\left(3 \times 20.0 \mathrm{~mL}\right.$ ), dried over $\mathrm{Na}_{2} \mathrm{SO}_{4}$, filtered off and concentrated in vacuo. The residue was purified by column chromatography (petroleum ether as the eluent) to afford the product as colorless oil (1.6 g, $82 \%$ yield).

\section{General procedure $\mathbf{E}^{9}$ :}

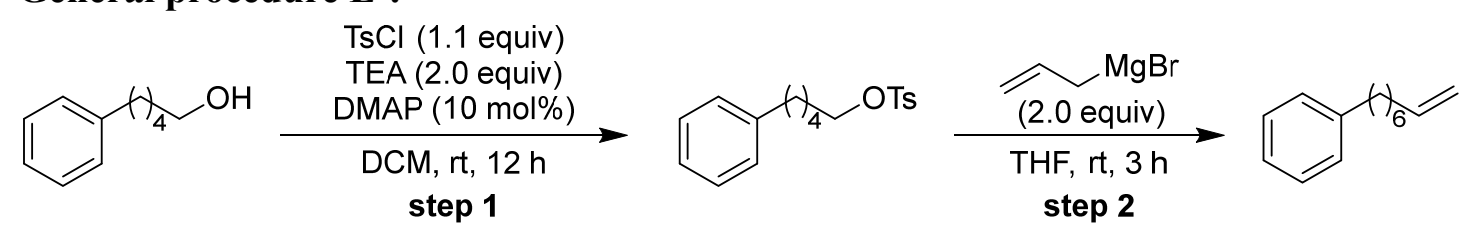

Step 1: In a $50.0 \mathrm{~mL}$ round bottom flask, a solution of alcohol $(5.0 \mathrm{mmol})$ in DCM $(20.0 \mathrm{~mL}), \mathrm{TsCl}$ (5.5 mmol), DMAP ( $0.5 \mathrm{mmol}, 10 \mathrm{~mol} \%)$ and $\mathrm{Et}_{3} \mathrm{~N}\left(10.0 \mathrm{mmol}, 2.0\right.$ equiv) were added at $0{ }^{\circ} \mathrm{C}$ and the reaction mixture was stirred for $12 \mathrm{~h}$ at room temperature under nitrogen atmosphere. After consumption of all starting material (as monitored by TLC), the reaction mixture was diluted with water $(20.0 \mathrm{~mL})$ and extracted with DCM $(3 \times 15.0 \mathrm{~mL})$. The combined organic layers were dried over anhydrous $\mathrm{Na}_{2} \mathrm{SO}_{4}$, filtered off and concentrated. The residue was purified by column chromatography (eluent: petroleum ether / ethyl acetate $=20 / 1$ ) to afford the product as colorless oil ( $1.1 \mathrm{~g}, 69 \%$ yield). 
Step 2: In a $50.0 \mathrm{~mL}$ round bottom flask fitted with a magnetic stir bar was added a solution of tosylate (from Step 1) $(636 \mathrm{mg}, 2.0 \mathrm{mmol})$ in THF $(10.0 \mathrm{~mL})$. Then a solution of allylmagnesium bromide ( $1 \mathrm{M}$ in THF, $4.0 \mathrm{mmol}, 2.0$ equiv) was added dropwise to the reaction mixture at $0{ }^{\circ} \mathrm{C}$ and stirred for $3 \mathrm{~h}$ at ambient temperature under nitrogen atmosphere. After consumption of all starting material (as monitored by TLC), the reaction mixture was quenched by the addition of saturated aqueous $\mathrm{NH}_{4} \mathrm{Cl}$. The obtained mixture was extracted with DCM $(3 \times 20.0 \mathrm{~mL})$, combined organic layers were dried over anhydrous $\mathrm{Na}_{2} \mathrm{SO}_{4}$, filtered off and concentrated under reduced pressure. The residue was purified by column chromatography using petroleum ether as eluent provided the desired product as a colorless oil (310 $\mathrm{mg}, 82 \%$ yield).

\section{Preparation of the substrate 2}

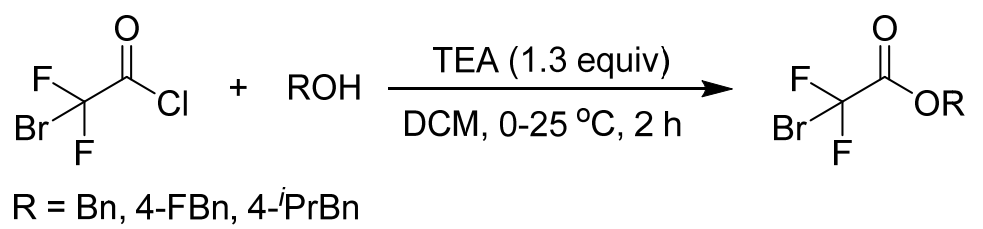

In a $15.0 \mathrm{~mL}$ Schlenk tube with a magnetic stirring bar were added $\mathrm{ROH}(2.0 \mathrm{mmol}), \mathrm{Et}_{3} \mathrm{~N}(2.6$ mmol, 1.3 equiv), and DCM $(3.0 \mathrm{~mL})$ under nitrogen atmosphere. This solution was cooled to $0{ }^{\circ} \mathrm{C}$, and the DCM $(2.0 \mathrm{~mL})$ solution of bromodifluoroacetyl chloride ( $2.2 \mathrm{mmol}, 1.1$ equiv) was added over $5 \mathrm{~min}$. After completion of the addition, the reaction mixture was warmed to room temperature and stirred for $2 \mathrm{~h}$. Then, the reaction mixture was quenched by saturated aqueous $\mathrm{NH}_{4} \mathrm{Cl}$ and extracted with DCM $(3 \times 5.0 \mathrm{~mL})$. The combined organic phase was washed with brine and dried over anhydrous $\mathrm{Na}_{2} \mathrm{SO}_{4}$, filtered off and concentrated under reduced pressure. The residue was purified by column chromatography using petroleum ether as eluent provided the desired product as a colorless oil. ${ }^{13}$

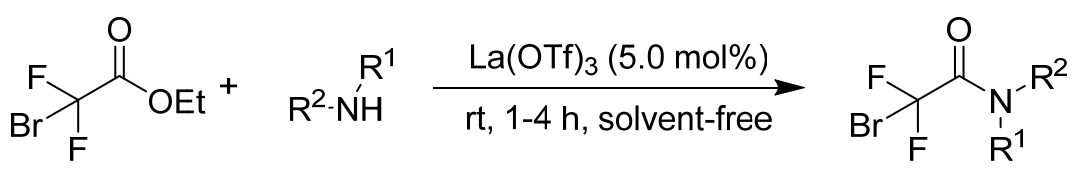<smiles>O=C(Nc1ccccc1)C(F)(F)C(F)(F)Br</smiles>

A $15.0 \mathrm{~mL}$ Schlenk tube equipped with a magnetic stir bar was charged with $\mathrm{La}(\mathrm{OTf})_{3}(0.25 \mathrm{mmol}$, $5 \mathrm{~mol} \%)$. The tube was backfilled with nitrogen, then ethyl bromodifluoroacetate $(6.0 \mathrm{mmol}, 1.2$ equiv) and amine $(5.0 \mathrm{mmol})$ were added. The mixture was stirred at the room temperature and monitored by TLC. After the amine was exhausted, the mixture was purified by silica gel column chromatography to give the target amide. ${ }^{14}$ 


\section{Unsuccessful examples}

The reactions were carried out under the General Procedure.
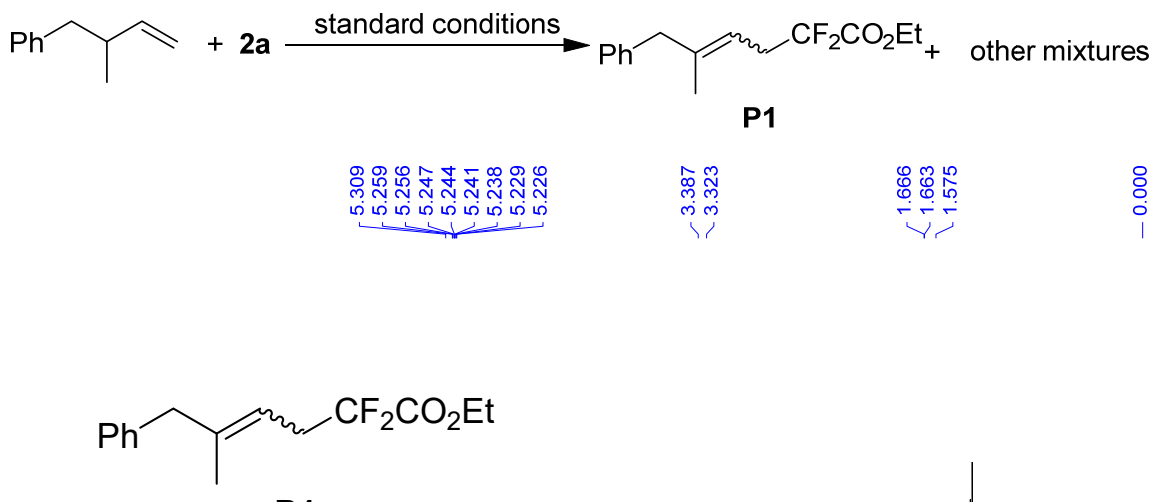

P1

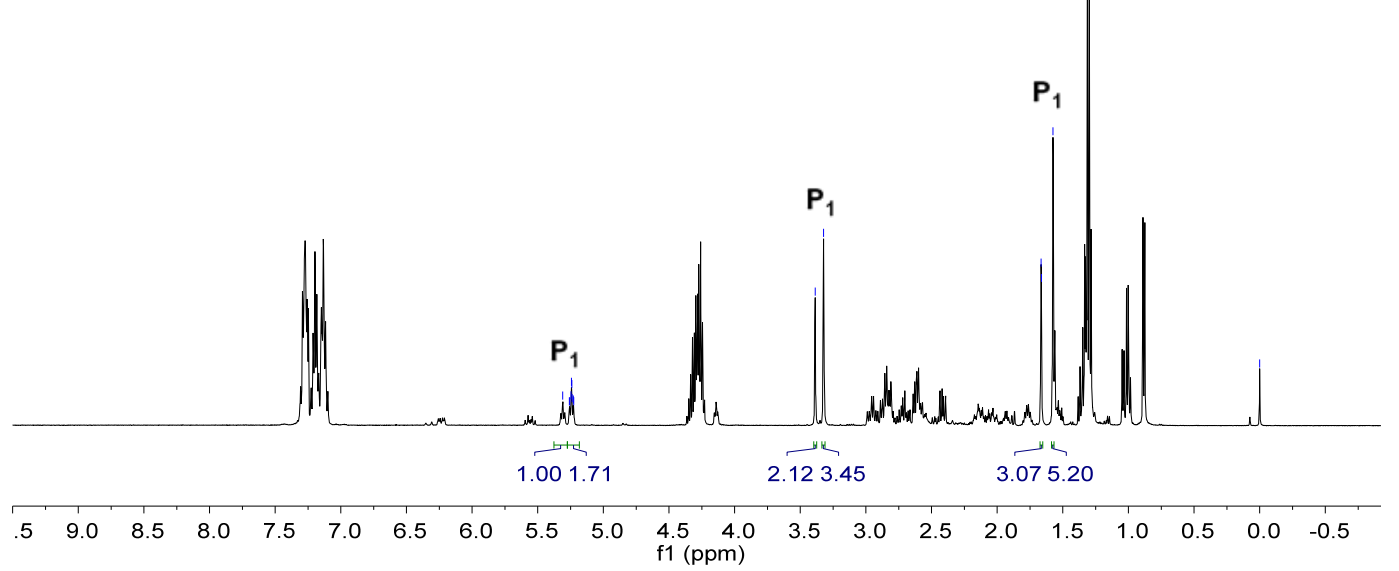

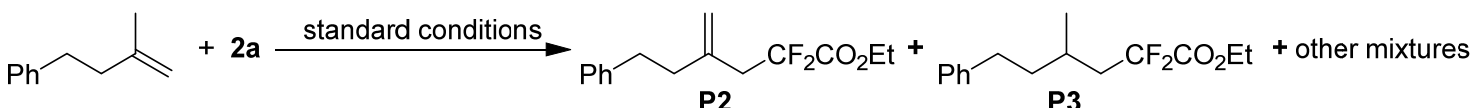



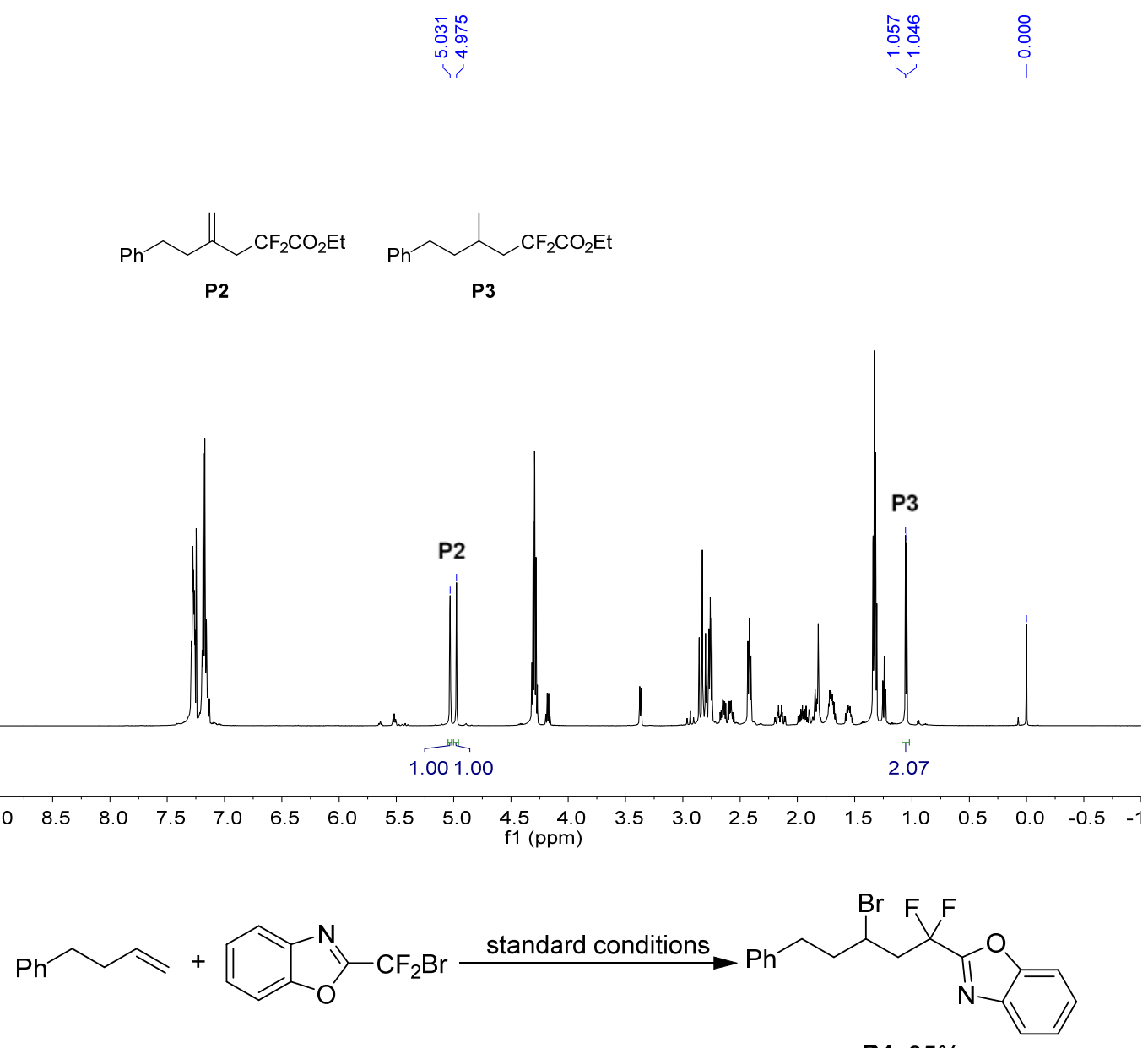

P4, 35\%

${ }^{1} \mathbf{H}$ NMR $\left(600 \mathrm{MHz}, \mathrm{CDCl}_{3}\right) \delta 7.82-7.80(\mathrm{~m}, 1 \mathrm{H}), 7.63-7.61(\mathrm{~m}, 1 \mathrm{H}), 7.49-7.46(\mathrm{~m}, 1 \mathrm{H}), 7.45-$ $7.42(\mathrm{~m}, 1 \mathrm{H}), 7.26-7.25(\mathrm{~m}, 2 \mathrm{H}), 7.19-7.18(\mathrm{~m}, 3 \mathrm{H}), 4.32-4.27(\mathrm{~m}, 1 \mathrm{H}), 3.27-3.18(\mathrm{~m}, 1 \mathrm{H}), 3.12$ $-3.03(\mathrm{~m}, 1 \mathrm{H}), 2.95-2.91(\mathrm{~m}, 1 \mathrm{H}), 2.81-2.76(\mathrm{~m}, 1 \mathrm{H}), 2.31-2.25(\mathrm{~m}, 1 \mathrm{H}), 2.24-2.18(\mathrm{~m}, 1 \mathrm{H})$.

HRMS (ESI-TOF) (m/z): calcd for $\mathrm{C}_{18} \mathrm{H}_{16} \mathrm{BrF}_{2} \mathrm{NNaO}^{+}\left([\mathrm{M}+\mathrm{Na}]^{+}\right)$, 402.0276; found, 402.0268 . 

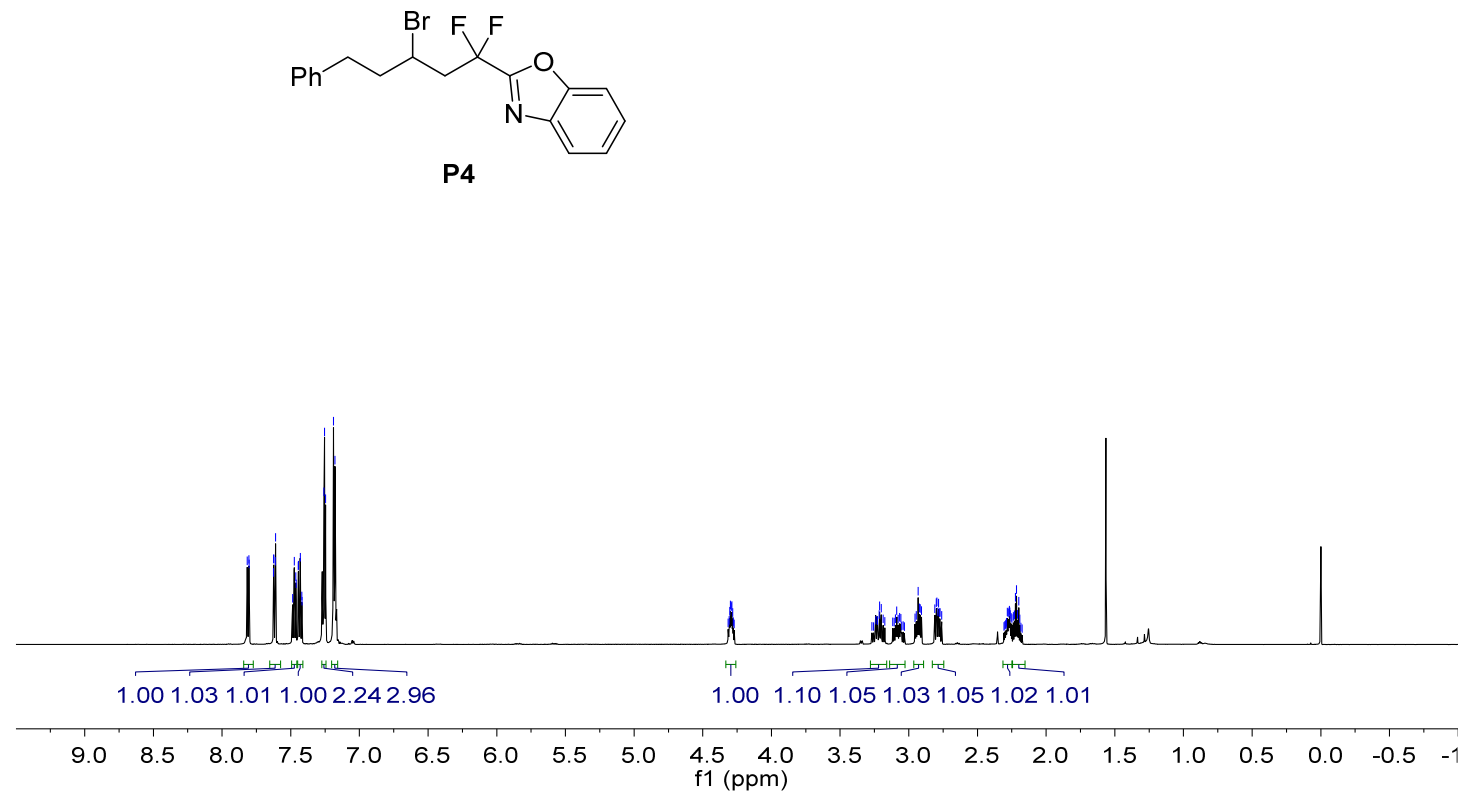


\section{Mechanistic Studies}

\section{The Radical Scavenger Experiment}

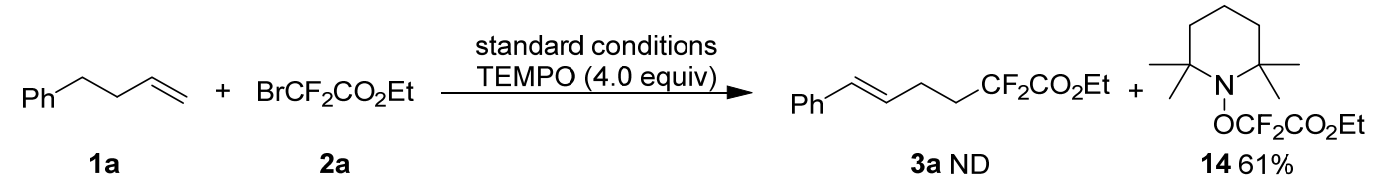

Into a nitrogen-filled glove box, a vial $(10.0 \mathrm{~mL})$ was charged with $\mathrm{Co}(\mathrm{dppbz}) \mathrm{Br}_{2}$, (13.3 $\mathrm{mg}, 10$ mol\%), Zn powder $(26.0 \mathrm{mg}, 0.4 \mathrm{mmol})$ and $\mathrm{CH}_{3} \mathrm{CN}(2.0 \mathrm{~mL})$. The reaction mixture was stirred at room temperature for $5 \mathrm{~min}$, then $\mathrm{Ni}(\mathrm{dtbbpy}) \mathrm{Br}_{2}(4.9 \mathrm{mg}, 5 \mathrm{~mol} \%), \mathbf{1 a}(26.4 \mathrm{mg}, 0.2 \mathrm{mmol})$ and $\mathbf{2 a}$ $(0.4 \mathrm{mmol})$ were added. Finally, TEMPO $(0.8 \mathrm{mmol}, 4.0$ equiv $)$ was added to the mixture. The vial was removed from the glovebox and stirred at $40{ }^{\circ} \mathrm{C}$ for $24 \mathrm{~h}$. After the reaction finished that monitored by TLC, the reaction mixture was cooled to room temperature and quenched by water. The mixture was extracted with $\mathrm{Et}_{2} \mathrm{O}(3 \times 5.0 \mathrm{~mL})$. The combined organic phases were dried over anhydrous $\mathrm{Na}_{2} \mathrm{SO}_{4}$, filtered off and concentrated under reduced pressure. The residue was analyzed by ${ }^{1} \mathrm{H}$ NMR, the product 3a was not detected, then the residue was purified by column chromatography on silica gel with petroleum ether as eluent to give the corresponding products $\mathbf{1 4}$ with $61 \%$ yield $(34.0 \mathrm{mg}$ ) (in comparison with $\mathbf{2 a}$ ). The spectroscopic properties of this compound were consistent with the data available in the literature. ${ }^{15}$

${ }^{1} \mathbf{H}$ NMR $\left(500 \mathrm{MHz}, \mathrm{CDCl}_{3}\right) \delta 4.35(\mathrm{q}, J=7.0 \mathrm{~Hz}, 2 \mathrm{H}), 1.60-1.52(\mathrm{~m}, 5 \mathrm{H}), 1.39-1.35$ (m, 4H), 1.20 (t, $J=2.5 \mathrm{~Hz}, 6 \mathrm{H}), 1.17$ (s, 6H).

${ }^{13}$ C NMR $\left(151 \mathrm{MHz}, \mathrm{CDCl}_{3}\right) \delta 160.7(\mathrm{t}, J=42.7 \mathrm{~Hz}), 115.5(\mathrm{t}, J=271.6 \mathrm{~Hz}), 63.0,61.4,40.2,33.4(\mathrm{t}$, $J=4.4 \mathrm{~Hz}), 20.7,16.9,13.9$.

${ }^{19}$ F NMR $\left(471 \mathrm{MHz}, \mathrm{CDCl}_{3}\right) \delta-73.5(\mathrm{~s})$.
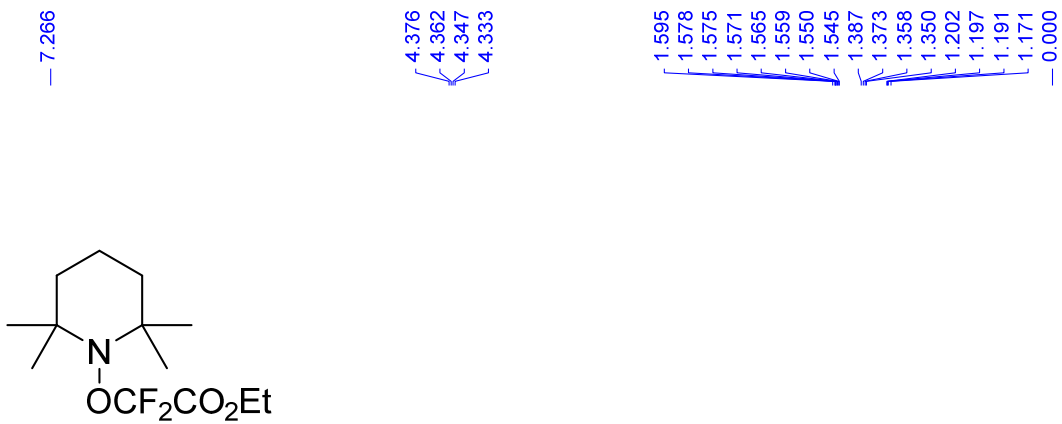

14

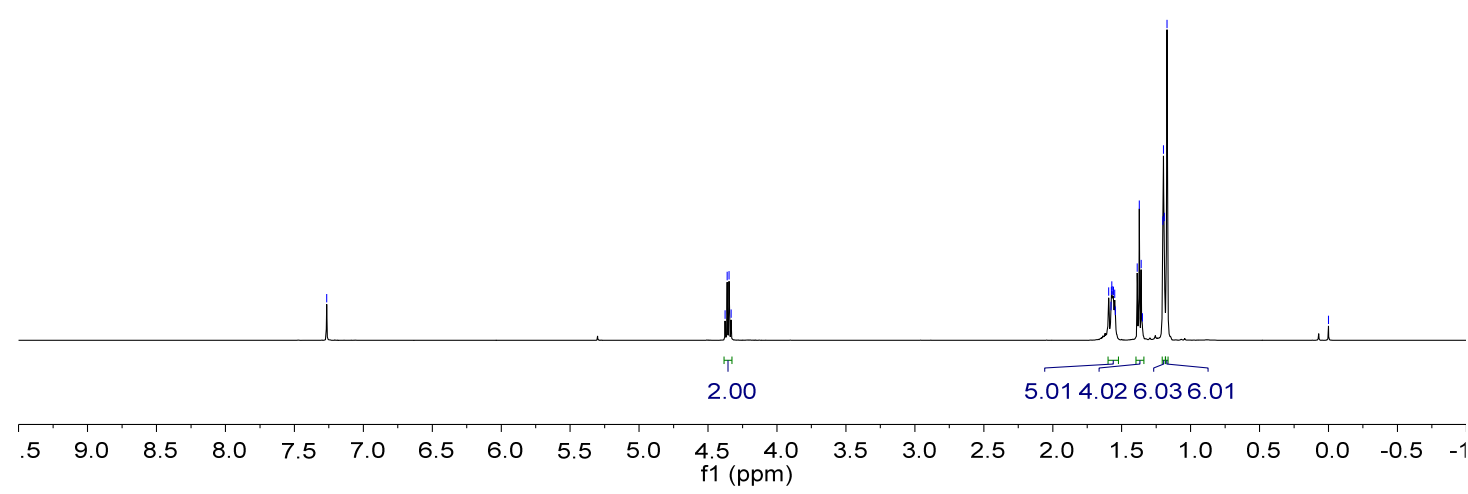




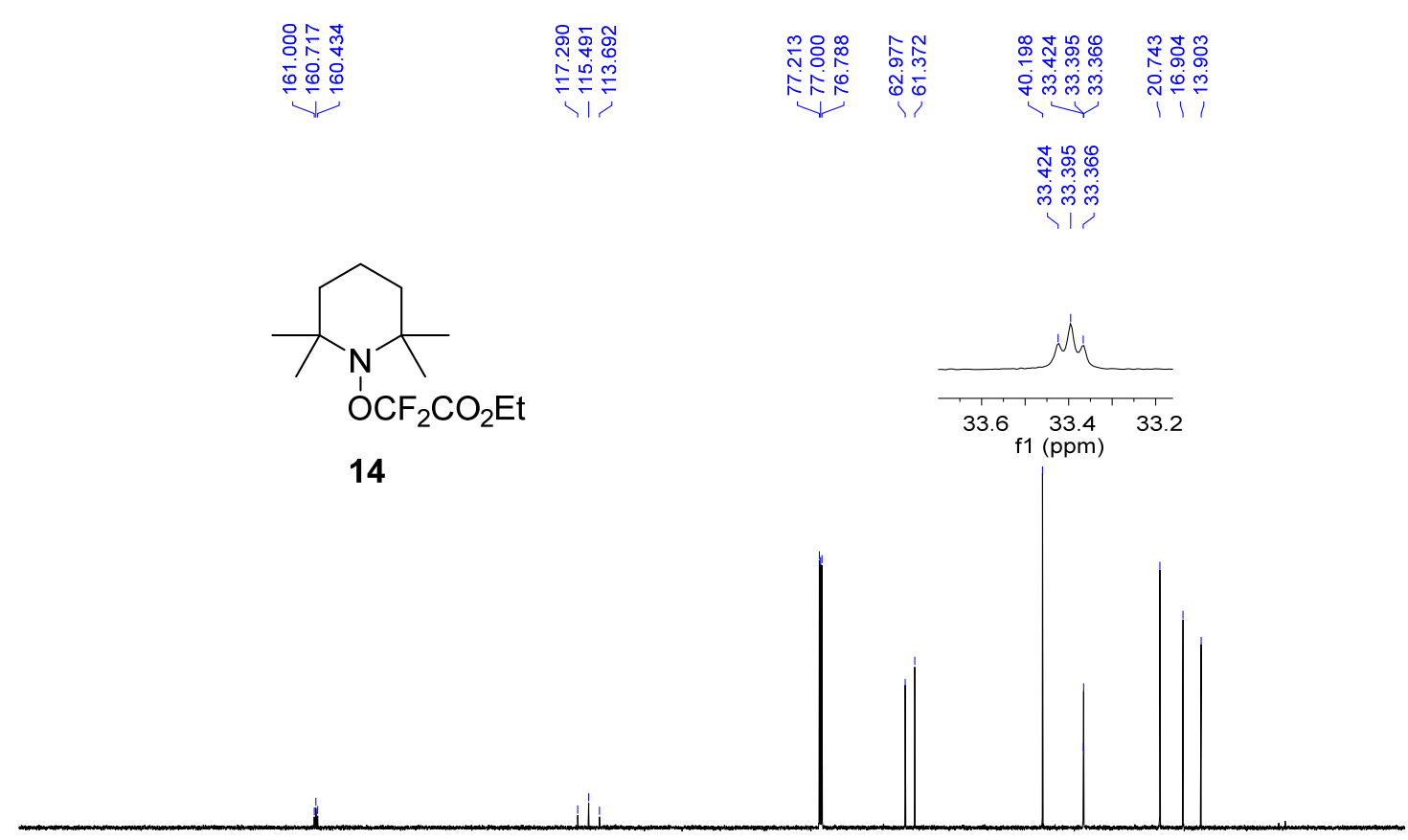

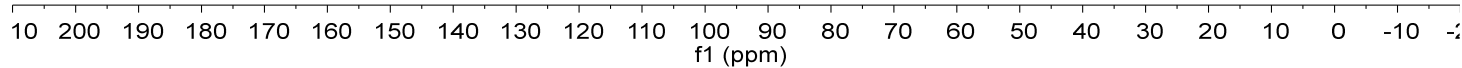

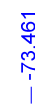
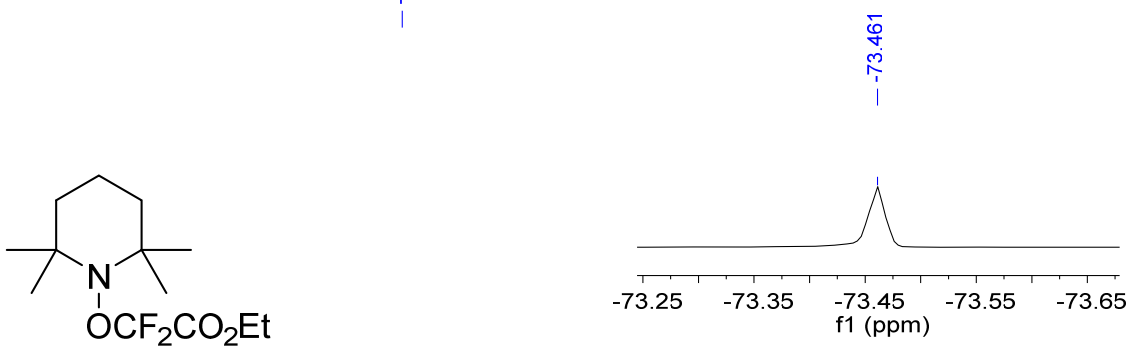

14

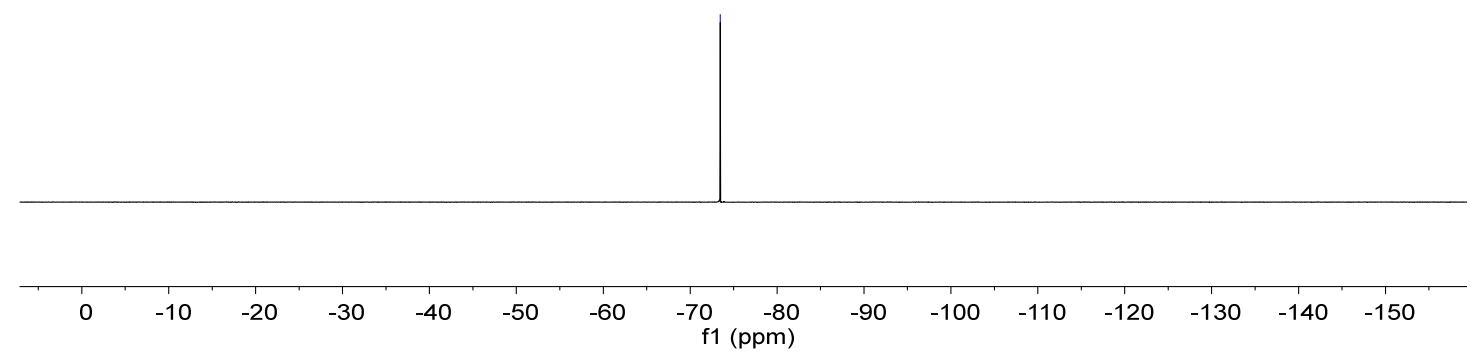


The Radical Clock Experiments

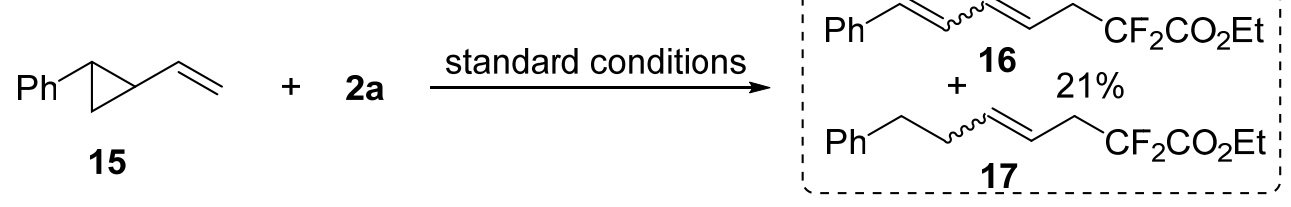

Into a nitrogen-filled glove box, a vial $(10.0 \mathrm{~mL})$ was charged with $\mathrm{Co}(\mathrm{dppbz}) \mathrm{Br}_{2}(13.3 \mathrm{mg}, 10 \mathrm{~mol} \%)$, Zn powder $(26.0 \mathrm{mg}, 0.4 \mathrm{mmol})$ and $\mathrm{CH}_{3} \mathrm{CN}(2.0 \mathrm{~mL})$. The reaction mixture was stirred at room temperature for $5 \mathrm{~min}$, then $\mathrm{Ni}(\mathrm{dtbbpy}) \mathrm{Br}_{2}(4.9 \mathrm{mg}, 5 \mathrm{~mol} \%), 15(28.8 \mathrm{mg}, 0.2 \mathrm{mmol})$ and $\mathbf{2 a}(0.4$ mmol) were added. The vial was removed from the glovebox and stirred at $40{ }^{\circ} \mathrm{C}$ for $24 \mathrm{~h}$. After the reaction finished that monitored by TLC, the reaction mixture was cooled to room temperature, then filtered through a pad of silica gel and concentrated under reduced pressure. Then the residue was purified by column chromatography on silica gel with petroleum ether as eluent to give the corresponding products $\mathbf{1 6}$ and $\mathbf{1 7}$ with $21 \%$ yield totally (16 and $\mathbf{1 7}$ were could not be readily separated by silica gel chromatography).

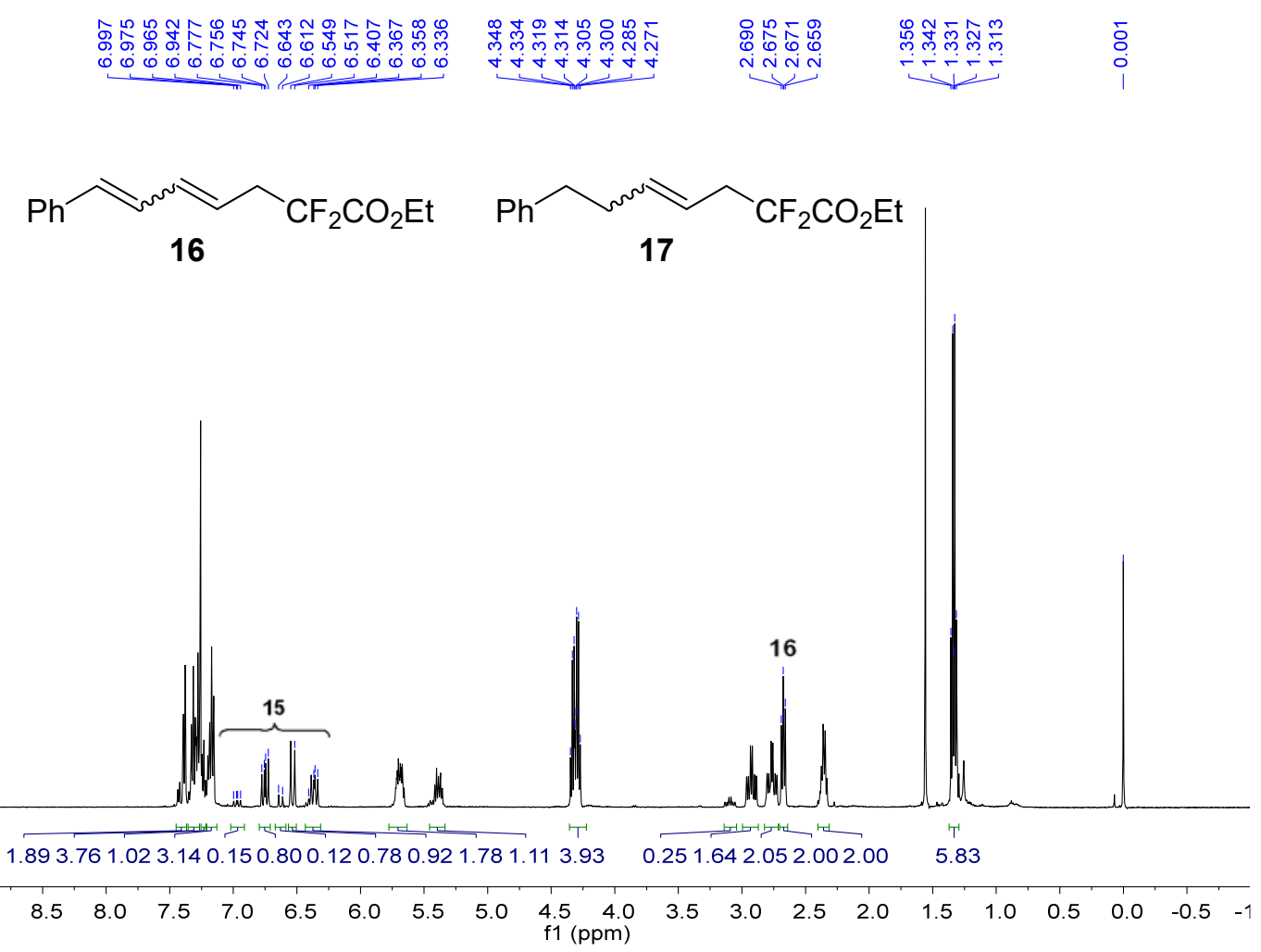

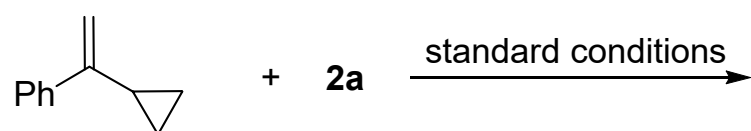

18

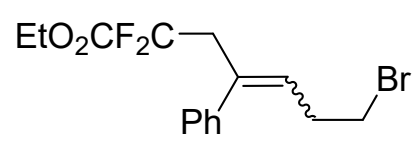

$19,18 \%(Z: E=1: 3)$

Into a nitrogen-filled glove box, a vial $(10.0 \mathrm{~mL})$ was charged with $\mathrm{Co}(\mathrm{dppbz}) \mathrm{Br}_{2}(13.3 \mathrm{mg}, 10 \mathrm{~mol} \%)$, $\mathrm{Zn}$ powder $(26.0 \mathrm{mg}, 0.4 \mathrm{mmol})$ and $\mathrm{CH}_{3} \mathrm{CN}(2.0 \mathrm{~mL})$. The reaction mixture was stirred at room temperature for $5 \mathrm{~min}$, then $\mathrm{Ni}(\mathrm{dtbbpy}) \mathrm{Br}_{2}(4.9 \mathrm{mg}, 5 \mathrm{~mol} \%), \mathbf{1 8}(28.8 \mathrm{mg}, 0.2 \mathrm{mmol})$ and $\mathbf{2 a}(0.4$ mmol) were added. The vial was removed from the glovebox and stirred at $40^{\circ} \mathrm{C}$ for $24 \mathrm{~h}$. After the 
reaction finished that monitored by TLC, the reaction mixture was cooled to room temperature then filtered through a pad of silica gel and concentrated under reduced pressure. Then the residue was purified by column chromatography on silica gel with petroleum ether as eluent to give the corresponding product 19 with $18 \%$ yield. The spectroscopic properties of this compound were consistent with the data available in the literature. ${ }^{16}$
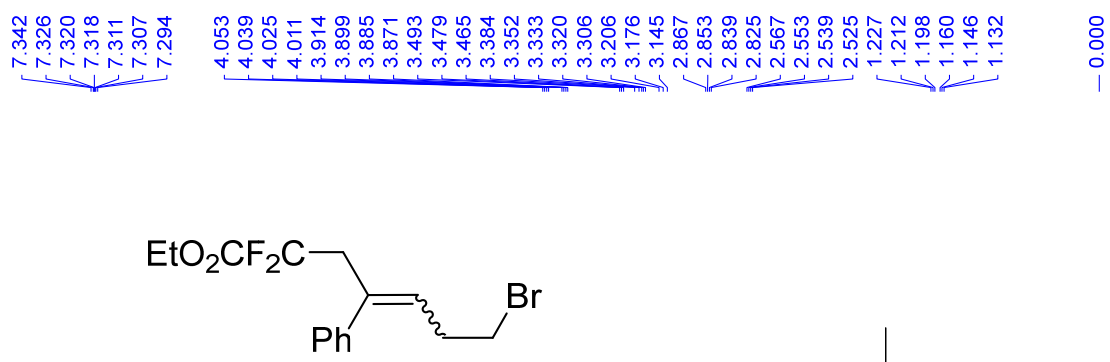

$19, Z: E=1: 3$

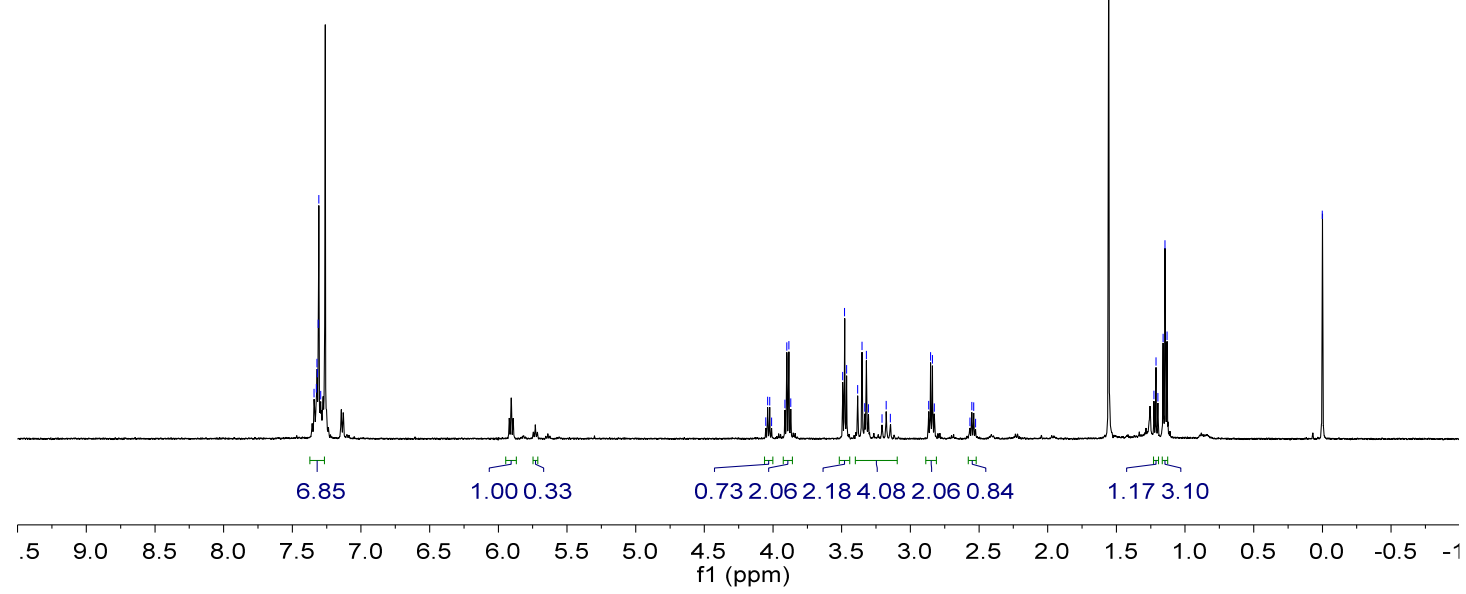




\section{Kinetic isotope experiments}

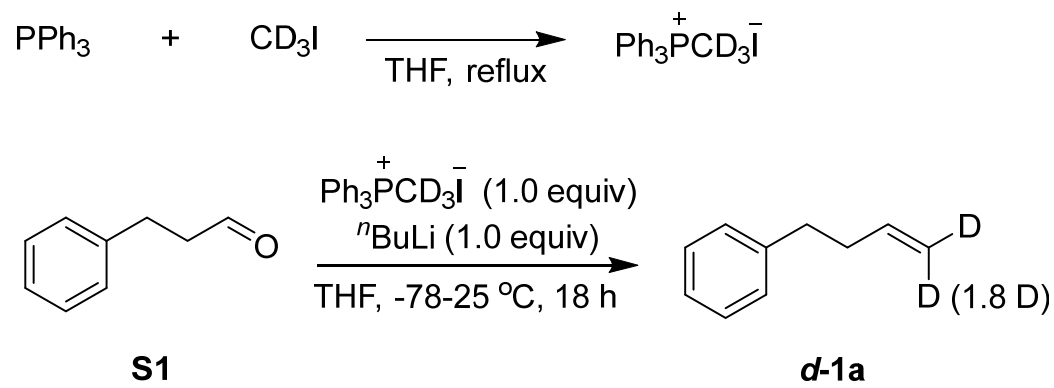

To a solution of triphenylphosphine $(10.0 \mathrm{mmol})$ in THF $(20.0 \mathrm{~mL})$ was added $\mathrm{CD}_{3} \mathrm{I}(12.0 \mathrm{mmol}$, 1.2 equiv) dropwise under nitrogen atmosphere. The solution was refluxed for $1 \mathrm{~h}$ and then cooled to room temperature and filtered. The precipitate was washed with xylene and dried under high vacuum to give the product as an off-white solid ( $3.9 \mathrm{~g}, 96 \%$ yield).

To a solution of methyl- $d_{3}$-triphenylphosphonium iodide $(5.0 \mathrm{mmol}, 1.0$ equiv) in THF (30.0 mL) at $-78{ }^{\circ} \mathrm{C}$ was added $n$-BuLi $(5.0 \mathrm{mmol}, 1.0$ equiv, $1.6 \mathrm{M}$ in hexane) dropwise over 5 min under nitrogen atmosphere. The solution was stirred at $-78{ }^{\circ} \mathrm{C}$ for $15 \mathrm{~min}$ and then warmed to room temperature and stirred for $30 \mathrm{~min}$. The reaction mixture was cooled back down to $-78{ }^{\circ} \mathrm{C}$ and 3 phenylpropanal $(5.0 \mathrm{mmol}$ ) was added dropwise over $5 \mathrm{~min}$. After $20 \mathrm{~min}$, the solution was warmed to room temperature and stirred for $18 \mathrm{~h}$. The reaction mixture was filtered through Celite and concentrated in vacuo. The residue was purified by flash column chromatography (petroleum ether as eluent) to give the product $\boldsymbol{d}-\mathbf{1 a}$ as a colorless oil $(0.5 \mathrm{~g}, 75 \%$ yield $) .{ }^{17}$

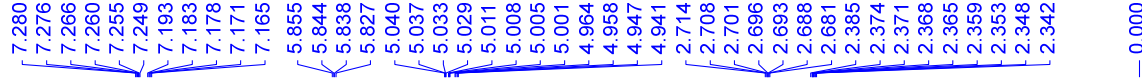<smiles>[2H]C(C)=CCCc1ccccc1</smiles>

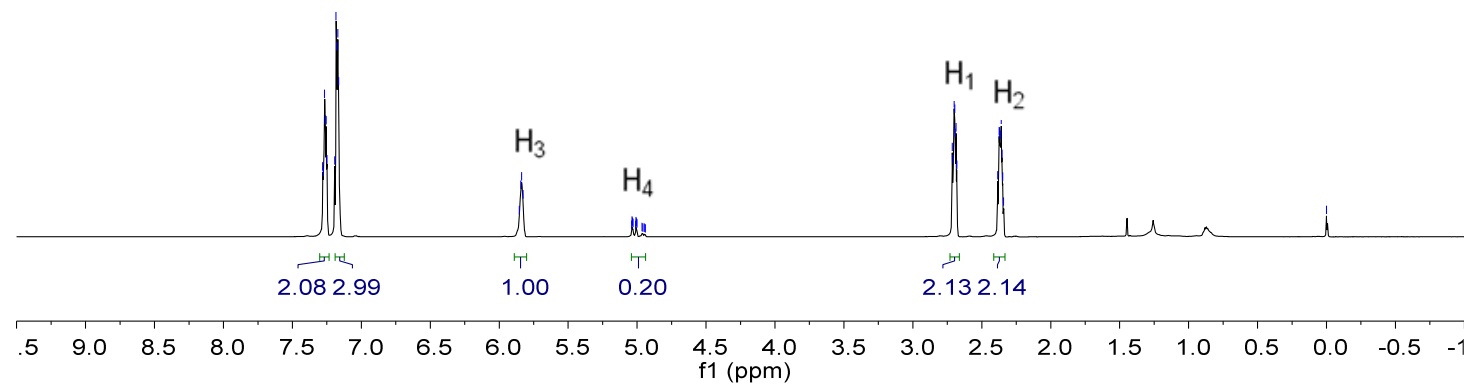




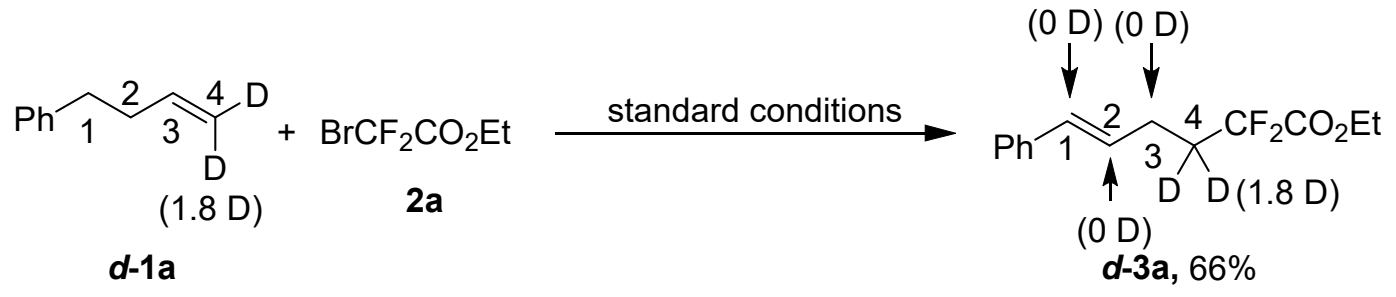

Into a nitrogen-filled glove box, a vial $(10.0 \mathrm{~mL})$ was charged $\mathrm{Co}(\mathrm{dppbz}) \mathrm{Br}_{2}(13.3 \mathrm{mg}, 10 \mathrm{~mol} \%), \mathrm{Zn}$ powder $(26.0 \mathrm{mg}, 0.4 \mathrm{mmol})$ and $\mathrm{CH}_{3} \mathrm{CN}(2.0 \mathrm{~mL})$. The reaction mixture was stirred at room temperature for $5 \mathrm{~min}$, then $\mathrm{Ni}(\mathrm{dtbbpy}) \mathrm{Br}_{2}(4.9 \mathrm{mg}, 5 \mathrm{~mol} \%), \boldsymbol{d}-\mathbf{1 a}(26.8 \mathrm{mg}, 0.2 \mathrm{mmol})$ and $\mathbf{2 a}(0.4$ mmol) were added. The vial was removed from the glovebox and stirred at $40{ }^{\circ} \mathrm{C}$ for $24 \mathrm{~h}$. After the reaction finished that monitored by TLC, the reaction mixture was cooled to room temperature and quenched by water. The mixture was extracted with $\mathrm{Et}_{2} \mathrm{O}(3 \times 5.0 \mathrm{~mL})$. The combined organic phases were dried over anhydrous $\mathrm{Na}_{2} \mathrm{SO}_{4}$, filtered off and the solvent was evaporated under vacuum. The residue was purified by flash column chromatography (petroleum ether / diethyl ether $=100 / 1)$ to give the corresponding product $\mathbf{d - 3 a}$.<smiles>[2H]C([2H])(CC=Cc1ccccc1)C(F)(F)C(=O)OCC</smiles>

d-3a

ethyl (E)-2,2-difluoro-6-phenylhex-5-enoate-3,3-d 2

${ }^{1} \mathbf{H}$ NMR $\left(600 \mathrm{MHz}, \mathrm{CDCl}_{3}\right) \delta 7.33(\mathrm{~d}, J=7.2 \mathrm{~Hz}, 2 \mathrm{H}), 7.30(\mathrm{t}, J=7.2 \mathrm{~Hz}, 2 \mathrm{H}), 7.21(\mathrm{t}, J=7.2 \mathrm{~Hz}, 1 \mathrm{H})$, $6.43(\mathrm{~d}, J=15.6 \mathrm{~Hz}, 1 \mathrm{H}), 6.18-6.13(\mathrm{~m}, 1 \mathrm{H}), 4.29$ (q, $J=7.2 \mathrm{~Hz}, 2 \mathrm{H}), 2.43-2.40$ (m, 2H), $2.27-2.21$ (m, $0.2 \mathrm{H}) 1.33(\mathrm{t}, J=7.2 \mathrm{~Hz}, 3 \mathrm{H})$.

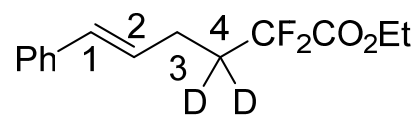

$d-3 a$

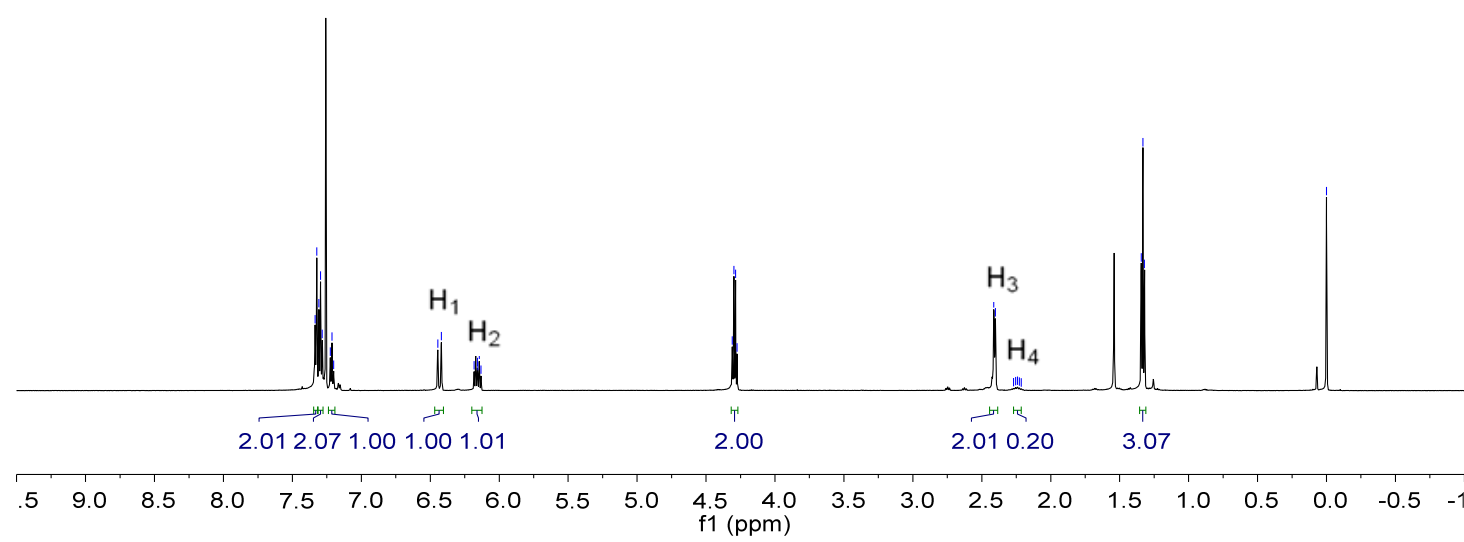




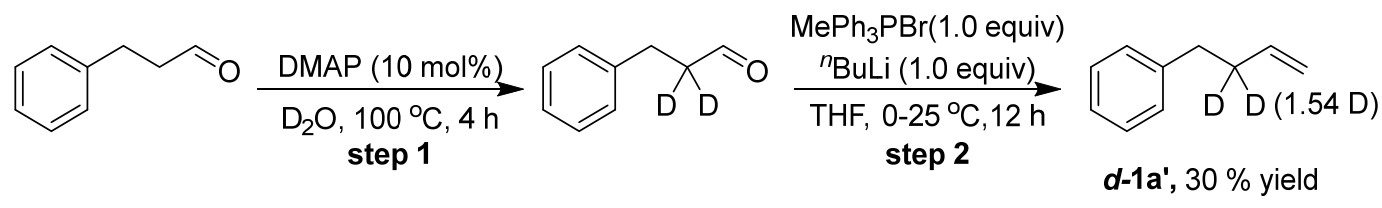

Step 1: In a dry $20.0 \mathrm{~mL}$ vial charged with Teflon-coated stir bar was added $\mathrm{D}_{2} \mathrm{O}(2.5 \mathrm{~mL}), 3$ phenylpropanal $(2.64 \mathrm{~mL}, 20.0 \mathrm{mmol})$, and 4-dimethylaminopyridine (244 $\mathrm{mg}, 2.0 \mathrm{mmol})$. The vessel was sealed and heated to $100^{\circ} \mathrm{C}$ for $4 \mathrm{~h}$. After cooling, the reaction was extracted with DCM $(3 \times 5.0 \mathrm{~mL})$, washed with $\mathrm{HCl}(1 \mathrm{M}, 1 \times 10.0 \mathrm{~mL})$, saturated $\mathrm{NaHCO}_{3}(1 \times 10.0 \mathrm{~mL})$, and saturated brine $(1 \times 10.0 \mathrm{~mL})$, dried over anhydrous $\mathrm{Na}_{2} \mathrm{SO}_{4}$, filtered off and concentrated under reduced pressure. The aldehyde was used directly in the next step.

Step 2: To a dry $250 \mathrm{~mL}$ round bottom flask containing a Teflon-coated stir bar was added methyltriphenylphosphonium bromide $(1.79 \mathrm{~g}, 5.0 \mathrm{mmol})$. THF $(60.0 \mathrm{~mL})$ was added, and the flask cooled to $0{ }^{\circ} \mathrm{C}$. ${ }^{n} \mathrm{BuLi}(1.6 \mathrm{M}$ in hexanes, $5.0 \mathrm{mmol})$ was added dropwise. The reaction was stirred at $0{ }^{\circ} \mathrm{C}$ for $30 \mathrm{~min}$, then aldehyde (from Step 1) was added dropwise and the reaction allowed to warm to room temperature. The reaction was stirred for $12 \mathrm{~h}$ at room temperature, then quenched by addition of saturated aqueous $\mathrm{NH}_{4} \mathrm{Cl}$, and extracted with diethyl ether $(3 \times 50.0 \mathrm{~mL})$, the combined organic layers were dried over anhydrous $\mathrm{Na}_{2} \mathrm{SO}_{4}$, filtered off and concentrated under reduced pressure. The residue was purified by flash column chromatography (petroleum ether as eluent) to give the product $\boldsymbol{d}-\mathbf{1} \mathbf{a}$ ' as a colorless oil in $30 \%$ overall yield. ${ }^{18}$

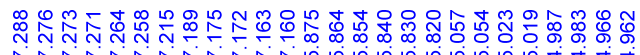

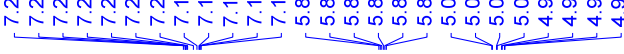

$\overbrace{1}^{1} x_{D}^{2} \widehat{3}^{4}$

$d-1 a^{\prime}$

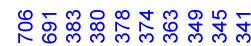

ง

:

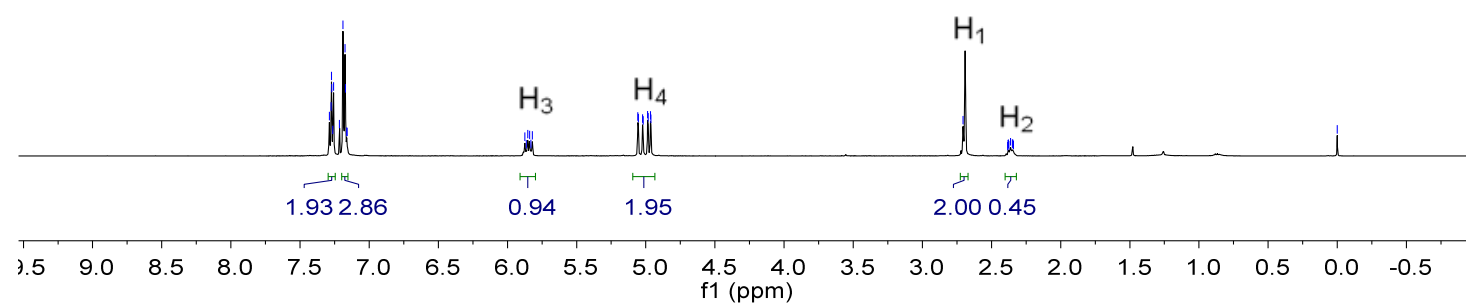




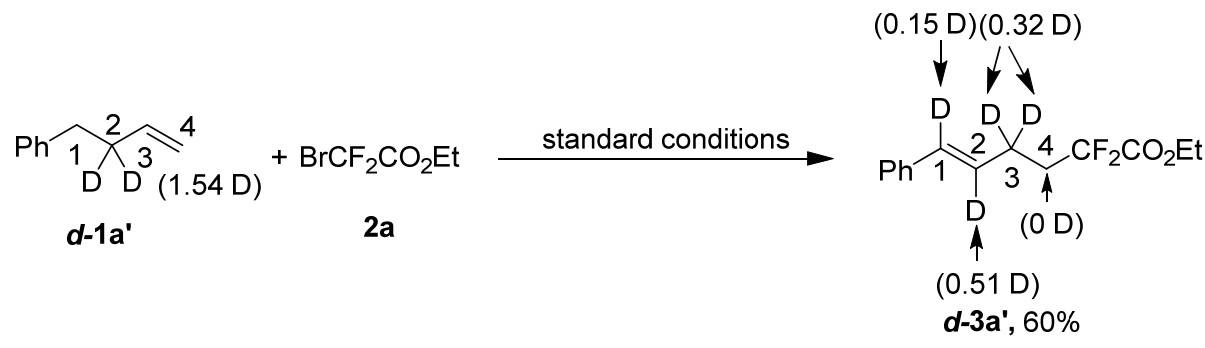

Into a nitrogen-filled glove box, a vial $(10.0 \mathrm{~mL})$ was charged with $\mathrm{Co}(\mathrm{dppbz}) \mathrm{Br}_{2}(13.3 \mathrm{mg}, 10 \mathrm{~mol} \%)$, Zn powder $(26.0 \mathrm{mg}, 0.4 \mathrm{mmol})$ and $\mathrm{CH}_{3} \mathrm{CN}(2.0 \mathrm{~mL})$. The reaction mixture was stirred at room temperature for $5 \mathrm{~min}$, then $\mathrm{Ni}(\mathrm{dtbbpy}) \mathrm{Br}_{2}(4.9 \mathrm{mg}, 5 \mathrm{~mol} \%), \boldsymbol{d}-\mathbf{1 a} \mathbf{a}^{\prime}(26.8 \mathrm{mg}, 0.2 \mathrm{mmol})$ and $\mathbf{2 a}$ $(0.4 \mathrm{mmol})$ were added. The vial was removed from the glovebox and stirred at $40^{\circ} \mathrm{C}$ for $24 \mathrm{~h}$. After the reaction finished that monitored by TLC, the reaction mixture was cooled to room temperature and quenched by water. The mixture was extracted with $\mathrm{Et}_{2} \mathrm{O}(3 \times 5.0 \mathrm{~mL})$. The combined organic phases were dried over anhydrous $\mathrm{Na}_{2} \mathrm{SO}_{4}$, filtered off and the solvent was evaporated under vacuum. The residue was purified by flash column chromatography (petroleum ether / diethyl ether $=100 / 1)$ to give the corresponding product $\mathbf{d}-\mathbf{3 a} \mathbf{a}^{\prime}(30.9 \mathrm{mg}, 60 \%$ yield $)$.<smiles>[2H]C(=C([2H])C([2H])([2H])C([2H])([18OH])CCC(=O)OCC)c1ccccc1</smiles>

ethyl (E)-2,2-difluoro-6-phenylhex-5-enoate-4,4,5,6-d 4

${ }^{1} \mathbf{H}$ NMR $\left(600 \mathrm{MHz}, \mathrm{CDCl}_{3}\right) \delta 7.33(\mathrm{~d}, J=7.2 \mathrm{~Hz}, 2 \mathrm{H}), 7.29(\mathrm{t}, J=7.2 \mathrm{~Hz}, 2 \mathrm{H}), 7.21(\mathrm{t}, J=7.2 \mathrm{~Hz}, 1 \mathrm{H})$, $6.43(\mathrm{~d}, J=15.6 \mathrm{~Hz}, 0.85 \mathrm{H}), 6.18-6.13(\mathrm{~m}, 0.49 \mathrm{H}), 4.29$ (q, $J=7.2 \mathrm{~Hz}, 2 \mathrm{H}), 2.44-2.40(\mathrm{~m}, 1.68 \mathrm{H})$, $2.30-2.21(\mathrm{~m}, 2 \mathrm{H}), 1.33(\mathrm{t}, J=7.2 \mathrm{~Hz}, 3 \mathrm{H})$. 
$(0.15 \mathrm{D})(0.32 \mathrm{D})$
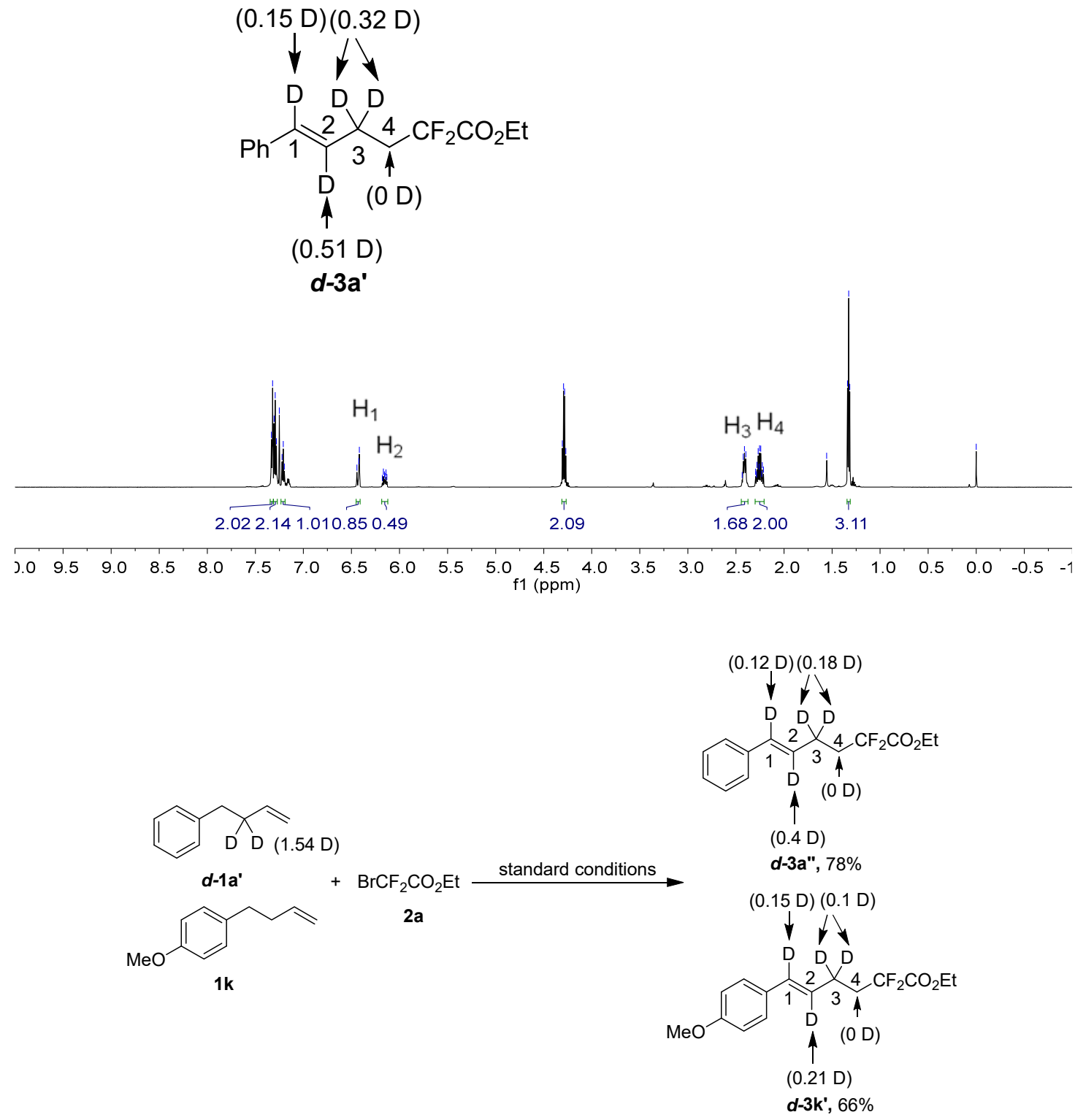

Into a nitrogen-filled glove box, a vial $(10.0 \mathrm{~mL})$ was charged with $\mathrm{Co}(\mathrm{dppbz}) \mathrm{Br}_{2},(26.6 \mathrm{mg}, 10$ mol\%), Zn powder (52.0 mg, $0.8 \mathrm{mmol})$ and $\mathrm{CH}_{3} \mathrm{CN}(4.0 \mathrm{~mL})$. The reaction mixture was stirred at room temperature for $5 \mathrm{~min}$, then $\mathrm{Ni}(\mathrm{dtbbpy}) \mathrm{Br}_{2}(9.8 \mathrm{mg}, 5 \mathrm{~mol} \%) \mathbf{d - 1 a}$ ' $(26.8 \mathrm{mg}, 0.2 \mathrm{mmol}), \mathbf{1 k}$ (32.4 $\mathrm{mg}, 0.2 \mathrm{mmol})$ and $\mathbf{2 a}(0.8 \mathrm{mmol})$ were added. The vial was removed from the glovebox and stirred at $40{ }^{\circ} \mathrm{C}$ for $24 \mathrm{~h}$. After the reaction finished that monitored by TLC, the reaction mixture was cooled to room temperature and quenched by water. The mixture was extracted with $\mathrm{Et}_{2} \mathrm{O}(3 \times$ $10.0 \mathrm{~mL}$ ). The combined organic phases were dried over anhydrous $\mathrm{Na}_{2} \mathrm{SO}_{4}$, filtered off and the solvent was evaporated under vacuum. The residue was purified by flash column chromatography (petroleum ether/ diethyl ether $=100 / 1)$ to give the corresponding product $\boldsymbol{d}-\mathbf{3 a}$ " $(40.2 \mathrm{mg}, 78 \%$ yield) and $\boldsymbol{d}-\mathbf{3 k} \mathbf{k}^{\prime}$ (38.0 mg, 66 \%yield). 
<smiles>[2H]/C(=C(\[2H])C([2H])([2H])CC(F)(F)F)c1ccccc1</smiles>

ethyl (E)-2,2-difluoro-6-phenylhex-5-enoate-4,4,5,6-d 4

${ }^{1} \mathbf{H}$ NMR $\left(500 \mathrm{MHz}, \mathrm{CDCl}_{3}\right) \delta 7.33(\mathrm{~d}, J=7.5 \mathrm{~Hz}, 2 \mathrm{H}), 7.31-7.28(\mathrm{~m}, 2 \mathrm{H}), 7.23-7.20(\mathrm{~m}, 1 \mathrm{H}), 6.43$ (d, $J=15.5 \mathrm{~Hz}, 0.88 \mathrm{H}), 6.19-613(\mathrm{~m}, 0.60 \mathrm{H}), 4.29$ (q, $J=7.0 \mathrm{~Hz}, 2 \mathrm{H}), 2.44-2.40(\mathrm{~m}, 1.82 \mathrm{H}), 2.31-$ $2.21(\mathrm{~m}, 2 \mathrm{H}), 1.33(\mathrm{t}, J=7.0 \mathrm{~Hz}, 3 \mathrm{H})$.<smiles>[2H]/C(C(=O)OCC)=C(\[2H])C([2H])([2H])CC(=O)OCC</smiles>

ethyl (E)-2,2-difluoro-6-(4-methoxyphenyl)hex-5-enoate-4,4,5,6-d 4

${ }^{1} \mathbf{H}$ NMR $\left(500 \mathrm{MHz}, \mathrm{CDCl}_{3}\right) \delta 7.26(\mathrm{~d}, J=8.5 \mathrm{~Hz}, 2 \mathrm{H}), 6.84(\mathrm{~d}, J=8.5 \mathrm{~Hz}, 2 \mathrm{H}), 6.37(\mathrm{~d}, J=15.5 \mathrm{~Hz}$, $0.85 \mathrm{H}), 6.04-5.98(\mathrm{~m}, 0.79 \mathrm{H}), 4.29$ (q, $J=7.0 \mathrm{~Hz}, 2 \mathrm{H}), 3.80(\mathrm{~s}, 3 \mathrm{H}), 2.41-2.37$ (m, 1.90H), $2.29-$ $2.19(\mathrm{~m}, 2 \mathrm{H}), 1.33(\mathrm{t}, J=7.5 \mathrm{~Hz}, 3 \mathrm{H})$.

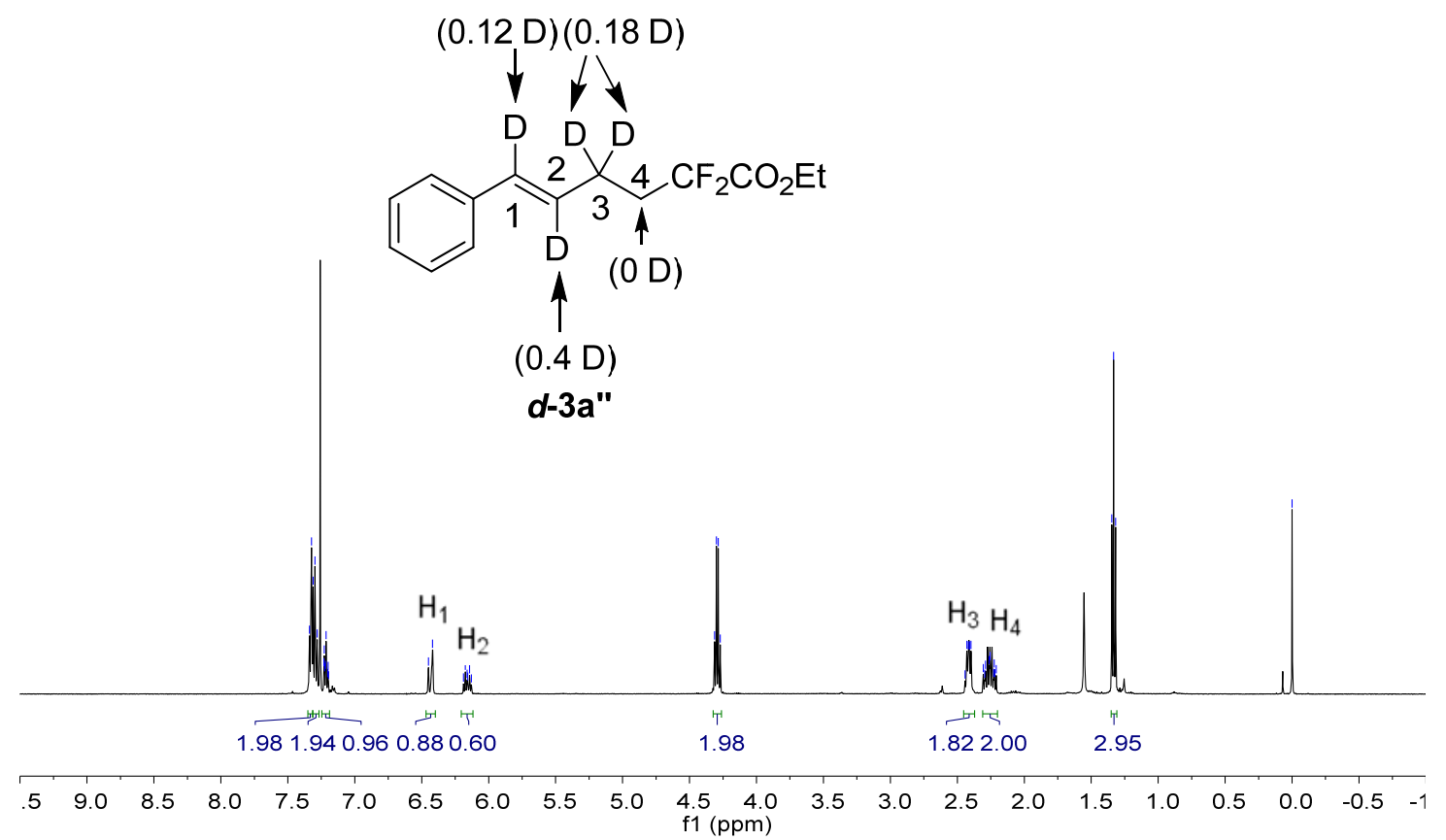




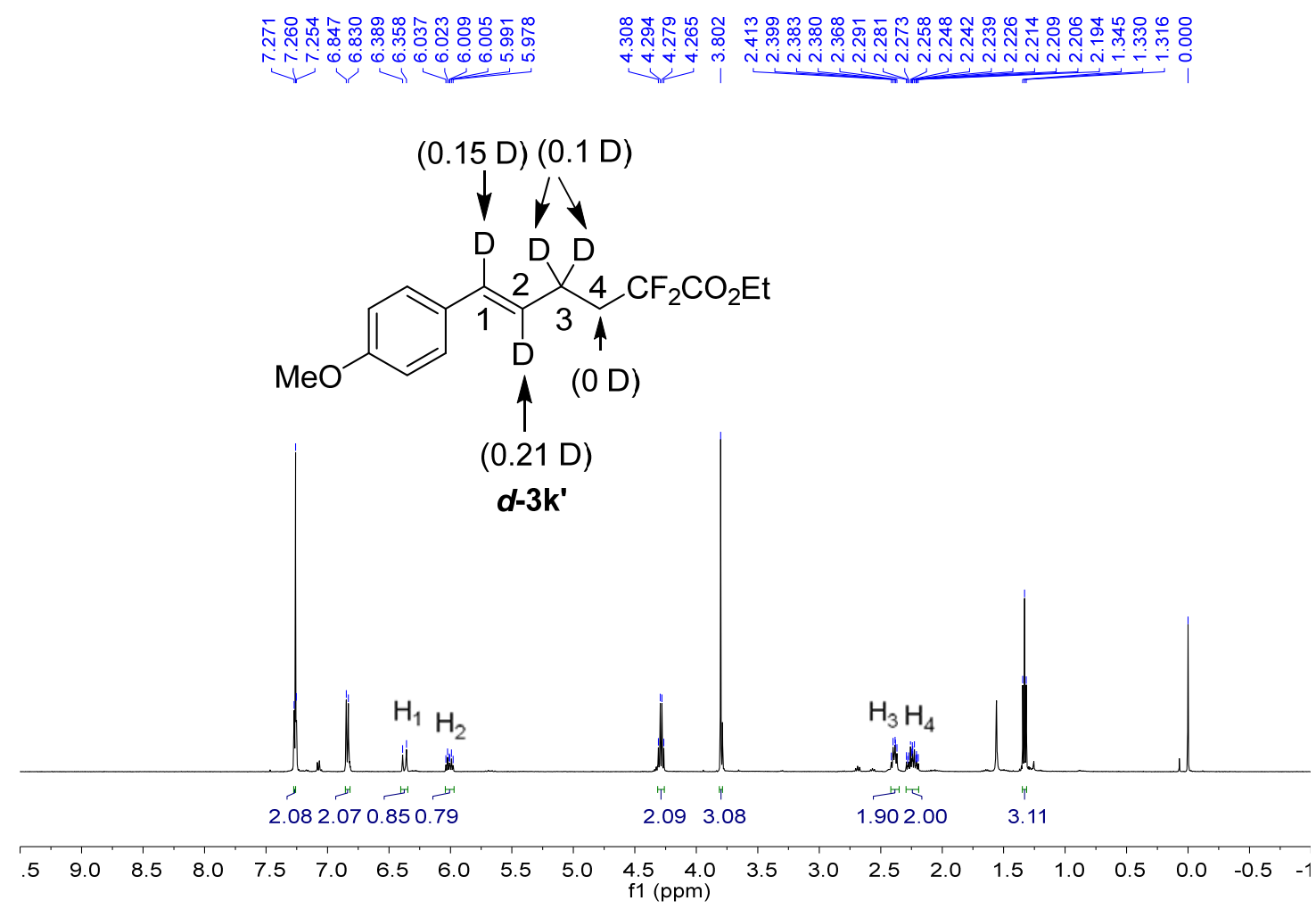

\section{Control Experiments}

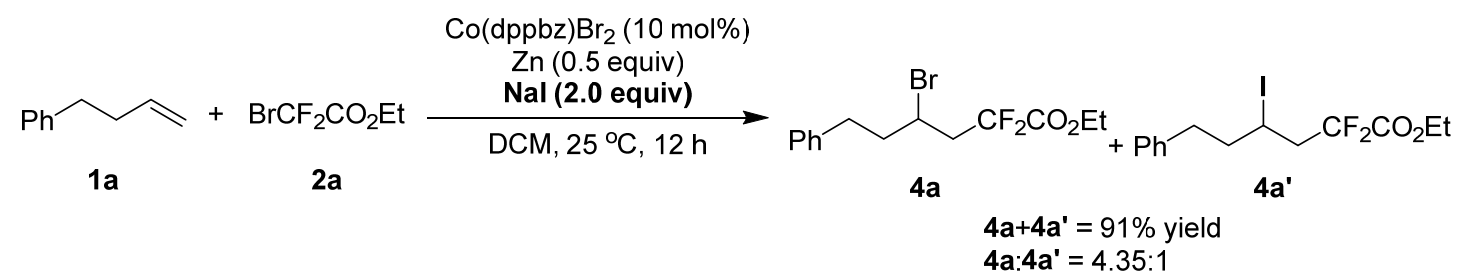

Into a nitrogen-filled glove box, a vial $(10.0 \mathrm{~mL})$ was charged with $\mathrm{Co}(\mathrm{dppbz}) \mathrm{Br}_{2}(13.3 \mathrm{mg}, 10 \mathrm{~mol} \%)$, Zn powder ( $6.5 \mathrm{mg}, 0.1 \mathrm{mmol}), \mathrm{NaI}$ (2.0 equiv) and anhydrous DCM $(2.0 \mathrm{~mL})$. The reaction mixture was stirred at room temperature for $5 \mathrm{~min}$, then $\mathbf{1 a}(0.2 \mathrm{mmol})$ and $\mathbf{2 a}(0.4 \mathrm{mmol})$ were added. The vial was removed from the glovebox and stirred at $25^{\circ} \mathrm{C}$ for $12 \mathrm{~h}$. After the reaction finished that monitored by TLC, the solution was passed through a short pad of silica gel and washed with DCM $(2 \times 10.0 \mathrm{~mL})$. The filtrate was concentrated in vacuo and then purified by silica gel column chromatography to give a mixture of $\mathbf{4 a}$ and $\mathbf{4 a}$ ', which could not separate effectively. The yield and ratio of $\mathbf{4 a}$ and $\mathbf{4 a}$ ' was determined by ${ }^{1} \mathrm{H}$ NMR (yield) and ${ }^{19} \mathrm{~F}$ NMR (ratio). ${ }^{19}$

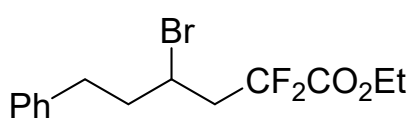

$4 a$

${ }^{1} \mathbf{H} \mathbf{N M R}\left(600 \mathrm{MHz}, \mathrm{CDCl}_{3}\right) \delta 7.29(\mathrm{t}, J=7.5 \mathrm{~Hz}, 2 \mathrm{H}), 7.21(\mathrm{t}, J=8.5 \mathrm{~Hz}, 3 \mathrm{H}), 4.31(\mathrm{q}, J=7.1 \mathrm{~Hz}, 2 \mathrm{H})$, $4.14-4.09(\mathrm{~m}, 1 \mathrm{H}), 2.93-2.82(\mathrm{~m}, 2 \mathrm{H}), 2.80-2.74(\mathrm{~m}, 1 \mathrm{H}), 2.71-2.61(\mathrm{~m}, 1 \mathrm{H}), 2.23-2.12(\mathrm{~m}, 2 \mathrm{H})$, $1.33(\mathrm{t}, J=7.2 \mathrm{~Hz}, 3 \mathrm{H})$.

${ }^{19}$ F NMR $\left(471 \mathrm{MHz}, \mathrm{CDCl}_{3}\right) \delta-101.27(\mathrm{dt}, J=265.6,14.1 \mathrm{~Hz}, 1 \mathrm{~F}),-106.88(\mathrm{dt}, J=265.6,16.5 \mathrm{~Hz}, 1 \mathrm{~F})$. 

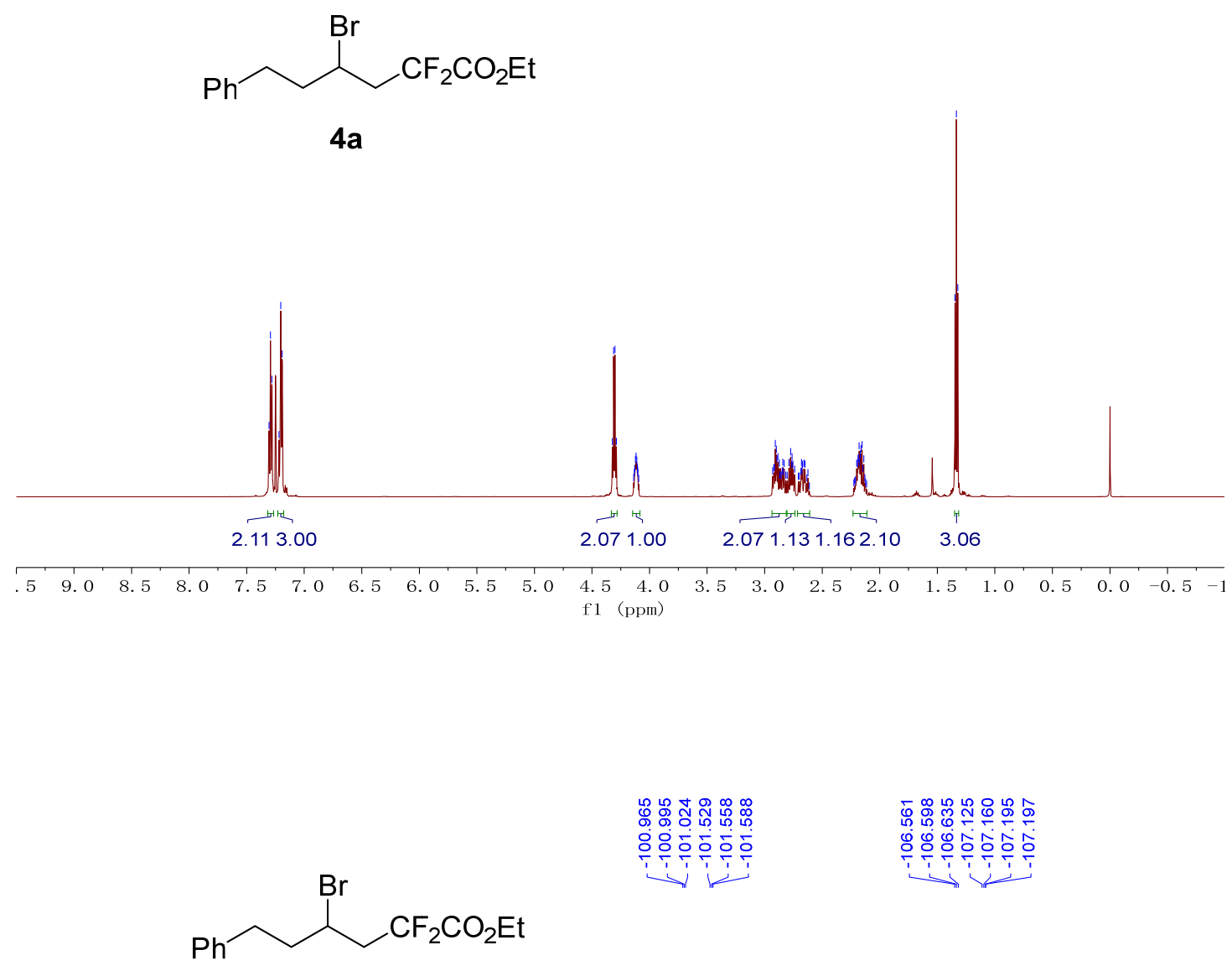

4a
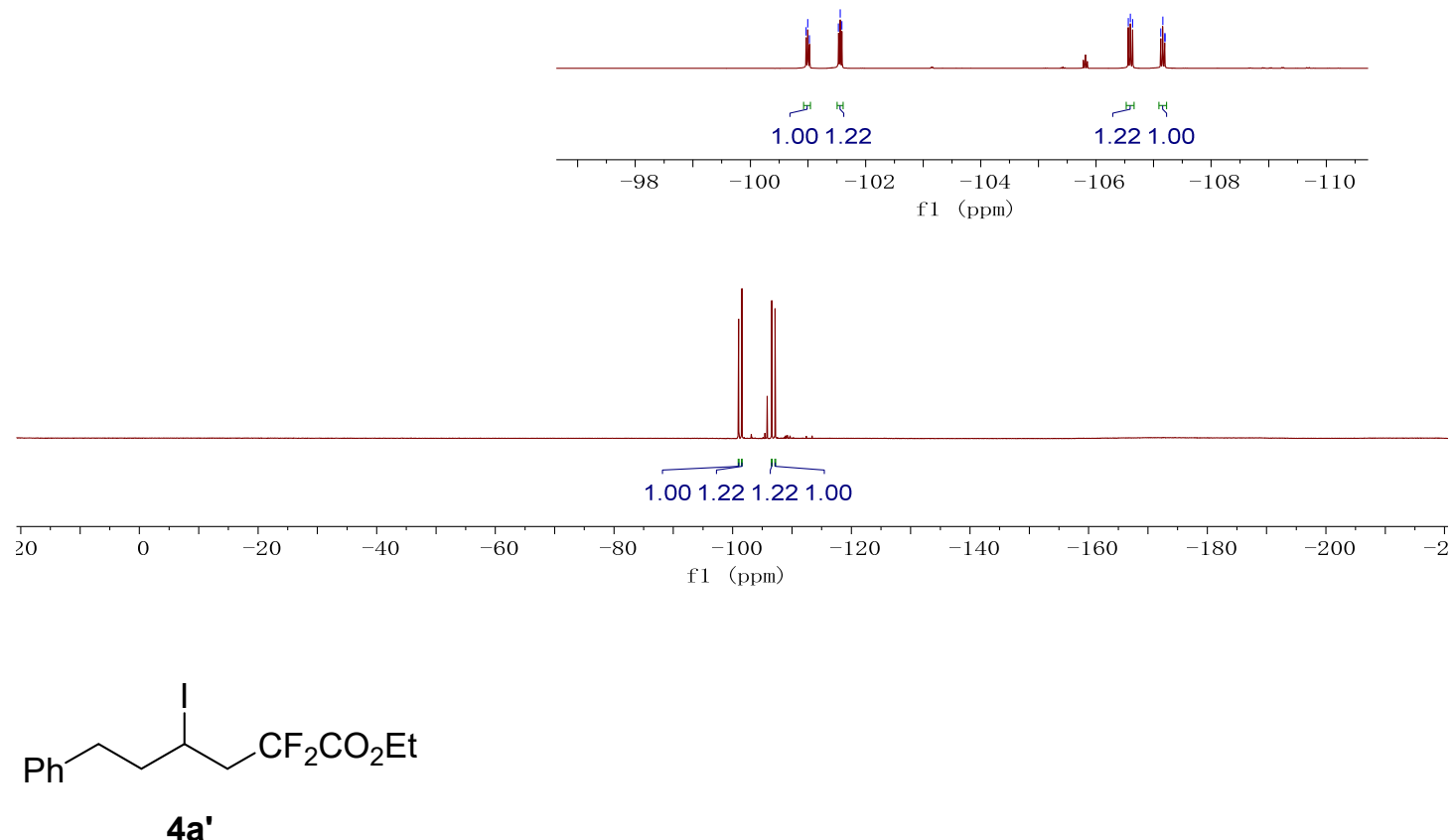
${ }^{1} \mathbf{H}$ NMR $\left(500 \mathrm{MHz}, \mathrm{CDCl}_{3}\right) \delta 7.29(\mathrm{t}, J=7.5 \mathrm{~Hz}, 2 \mathrm{H}), 7.21(\mathrm{t}, J=4.5 \mathrm{~Hz}, 3 \mathrm{H}), 4.29(\mathrm{q}, J=7.1 \mathrm{~Hz}, 2 \mathrm{H})$, $4.18-4.13(\mathrm{~m}, 1 \mathrm{H}), 3.01-2.87(\mathrm{~m}, 2 \mathrm{H}), 2.83-2.67(\mathrm{~m}, 2 \mathrm{H}), 2.16-2.01(\mathrm{~m}, 2 \mathrm{H}), 1.33(\mathrm{t}, J=7.0 \mathrm{~Hz}$, $3 \mathrm{H})$.

${ }^{19}$ F NMR $\left(471 \mathrm{MHz}, \mathrm{CDCl}_{3}\right) \delta-101.73(\mathrm{ddd}, J=220.0,16.0,10.4 \mathrm{~Hz}, 1 \mathrm{~F}),-106.40(\mathrm{dt}, J=220.0,14.6$ $\mathrm{Hz}, 1 \mathrm{~F})$.

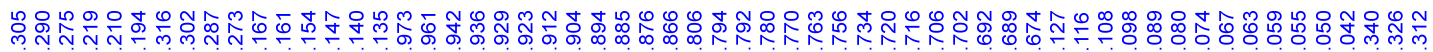

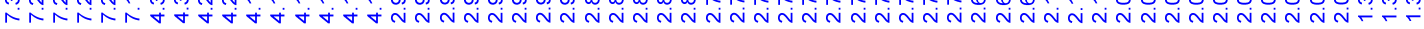
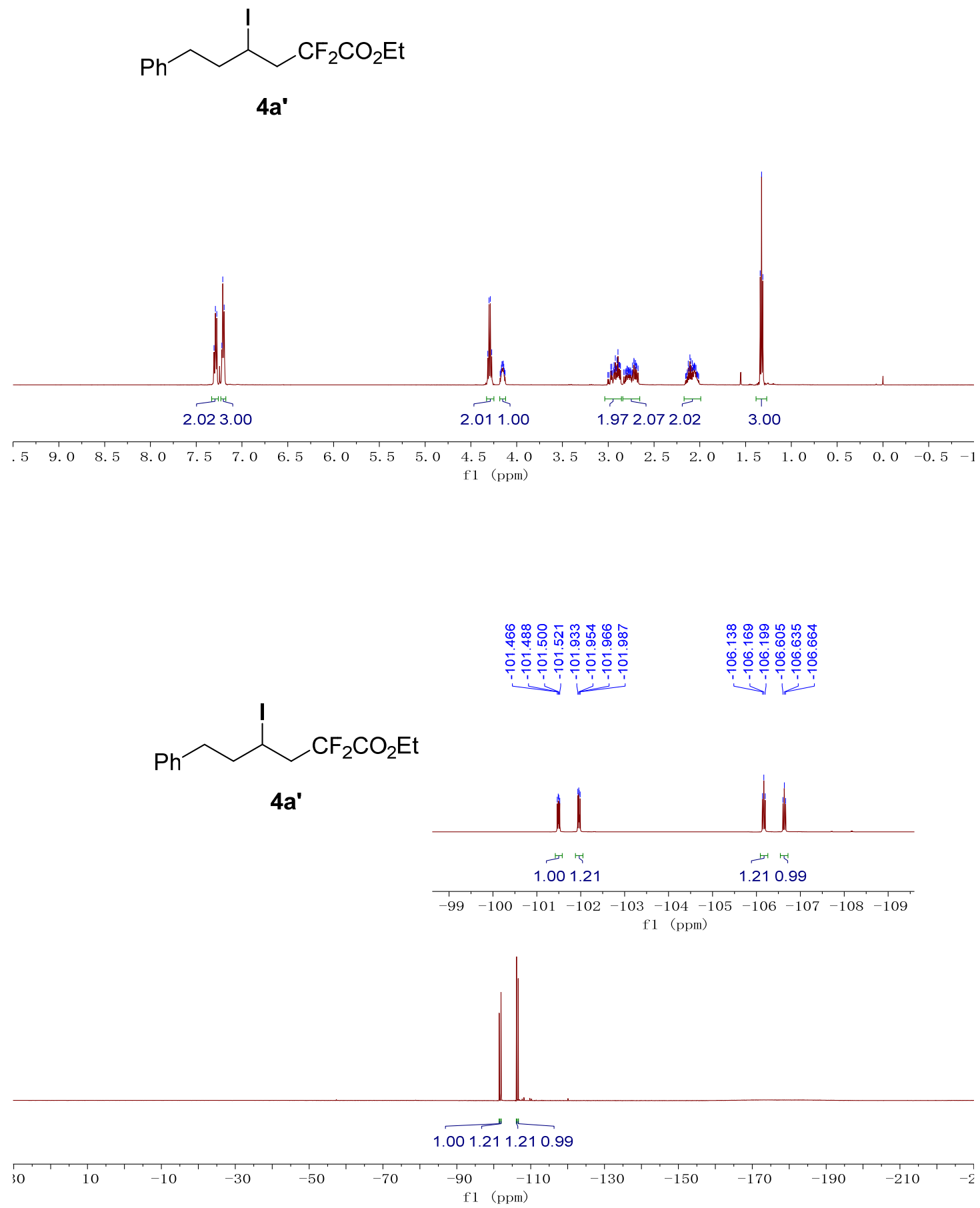


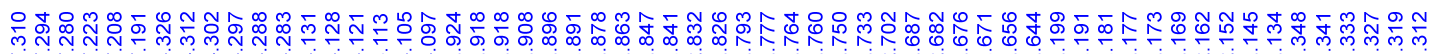

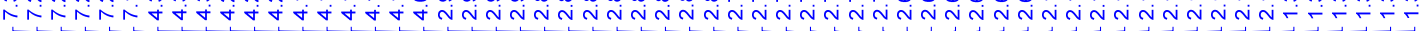

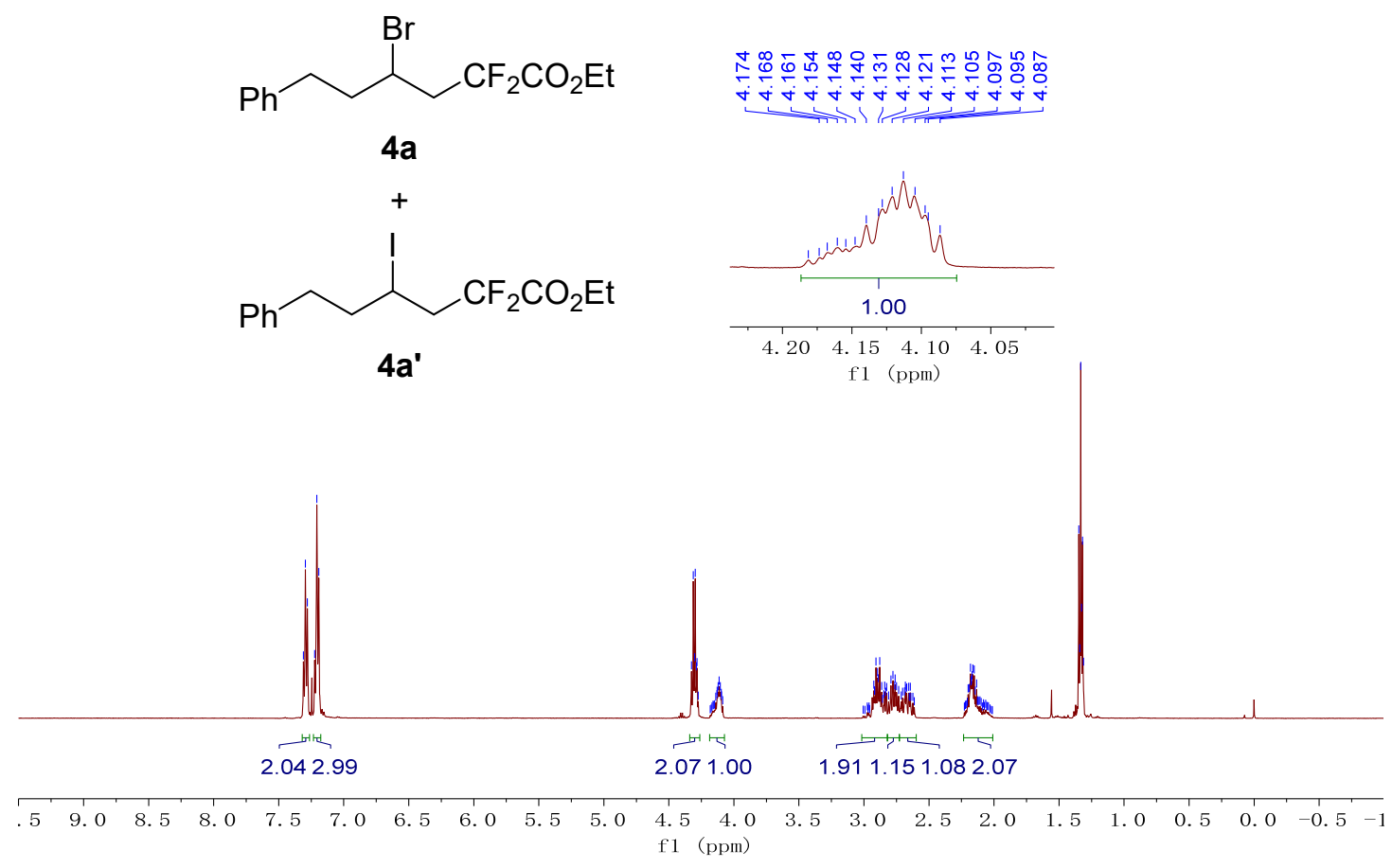<smiles>CCOC(=O)C(F)(F)CC(Br)CCc1ccccc1</smiles>

4a<smiles>CCOC(=O)[C-]CC([18F])CCc1ccccc1</smiles>

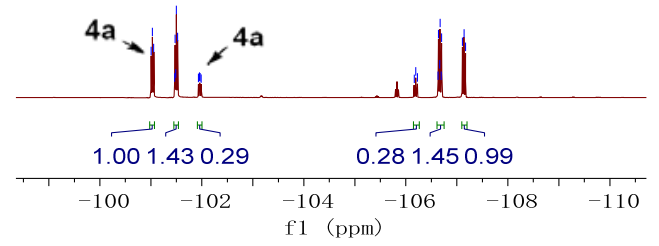

$4 a: 4 a^{\prime}=4.35: 1$

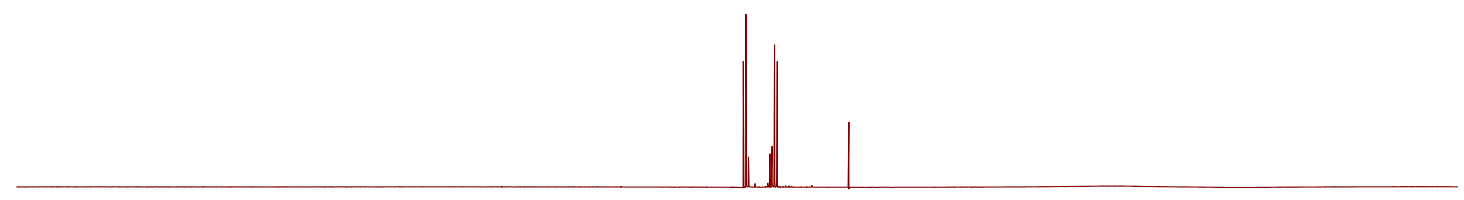

1.001 .430 .290 .281 .450 .99

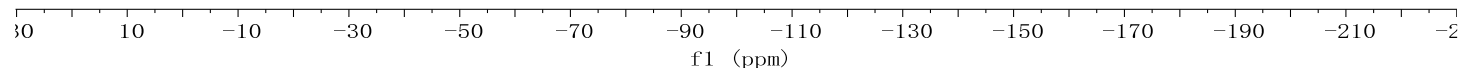




\section{Control Experiment with 6a}

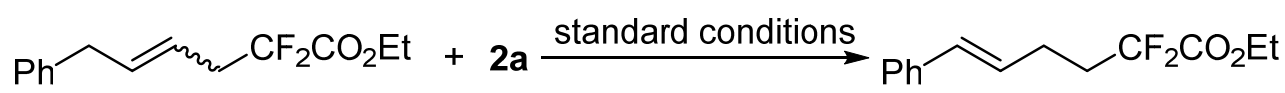

$6 \mathrm{a}$

3a, $46 \%$

Into a nitrogen-filled glove box, a vial $(10.0 \mathrm{~mL})$ was charged with $\mathrm{Co}(\mathrm{dppbz}) \mathrm{Br}_{2}(13.3 \mathrm{mg}, 10 \mathrm{~mol} \%)$, Zn powder $(26.0 \mathrm{mg}, 0.4 \mathrm{mmol})$ and $\mathrm{CH}_{3} \mathrm{CN}(2.0 \mathrm{~mL})$. The reaction mixture was stirred at room temperature for $5 \mathrm{~min}$, then $\mathrm{Ni}(\mathrm{dtbbpy}) \mathrm{Br}_{2}(4.9 \mathrm{mg}, 5 \mathrm{~mol} \%), \mathbf{6 a}(50.8 \mathrm{mg}, 0.2 \mathrm{mmol})$ and $\mathbf{2 a}(0.4$ mmol) were added. The vial was removed from the glovebox and stirred at $40{ }^{\circ} \mathrm{C}$ for $24 \mathrm{~h}$. The reaction mixture was cooled to room temperature, then filtered through a pad of silica gel and concentrated under reduced pressure. The yields determined by crude NMR using dibromomethane $\left(\mathrm{CH}_{2} \mathrm{Br}_{2}\right)$ as an internal standard.

The Time Course Study of 1a
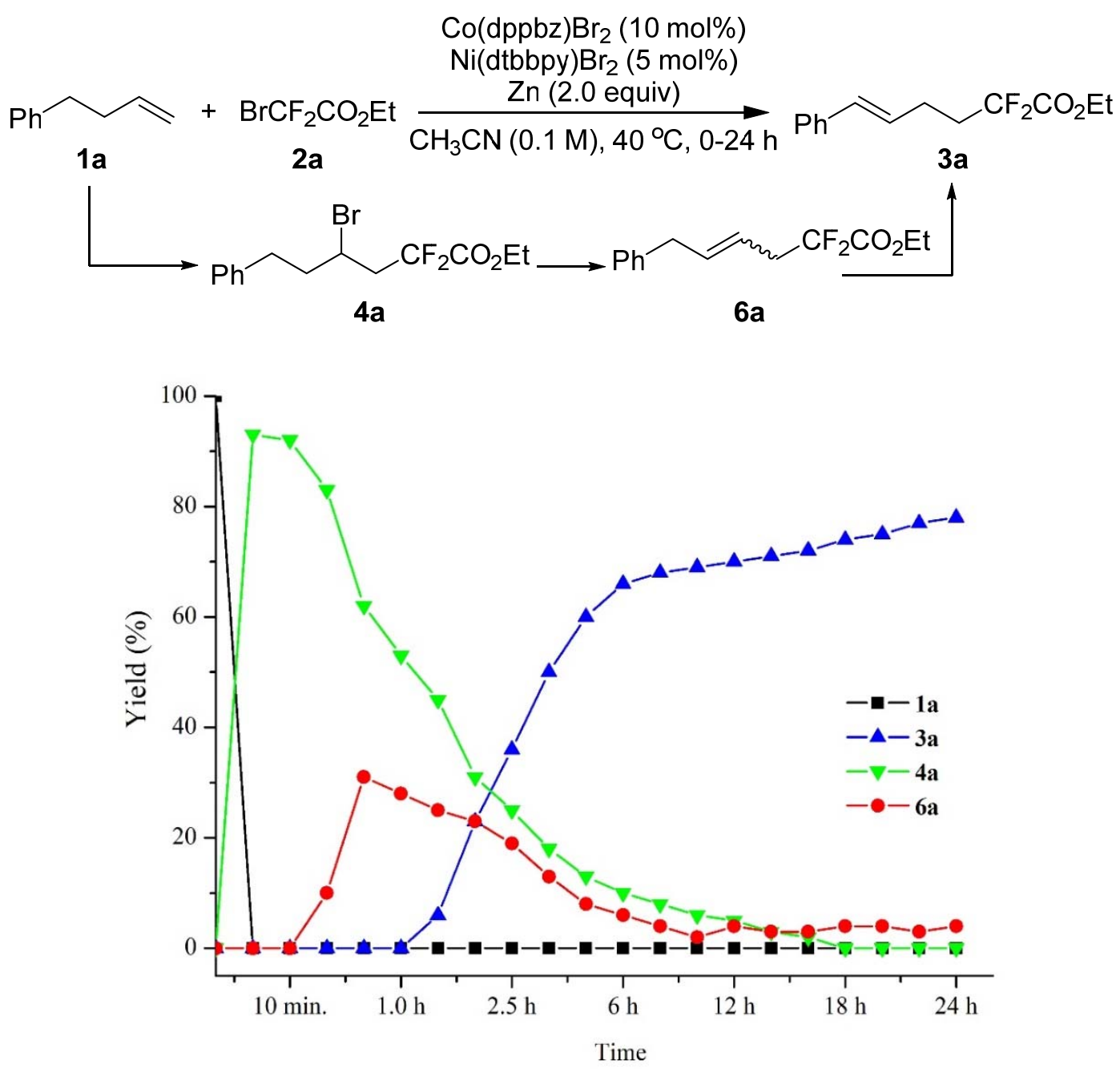

Conditions: 1a (0.1 mmol), 2 a ( $0.2 \mathrm{mmol}, 2.0$ equiv), $\mathrm{Co}(\mathrm{dppbz}) \mathrm{Br}_{2}(10 \mathrm{~mol} \%), \mathrm{Ni}(\mathrm{dtbbpy}) \mathrm{Br}_{2}(5$ mol\%), $\mathrm{Zn}\left(0.2 \mathrm{mmol}, 2.0\right.$ equiv), $\mathrm{CH}_{3} \mathrm{CN}(1.0 \mathrm{~mL}, 0.1 \mathrm{M}), 40{ }^{\circ} \mathrm{C}, 0-24 \mathrm{~h}$. The yields determined by crude NMR using dibromomethane $\left(\mathrm{CH}_{2} \mathrm{Br}_{2}\right)$ as an internal standard. 


\section{UV-Vis Absorption Spectra}

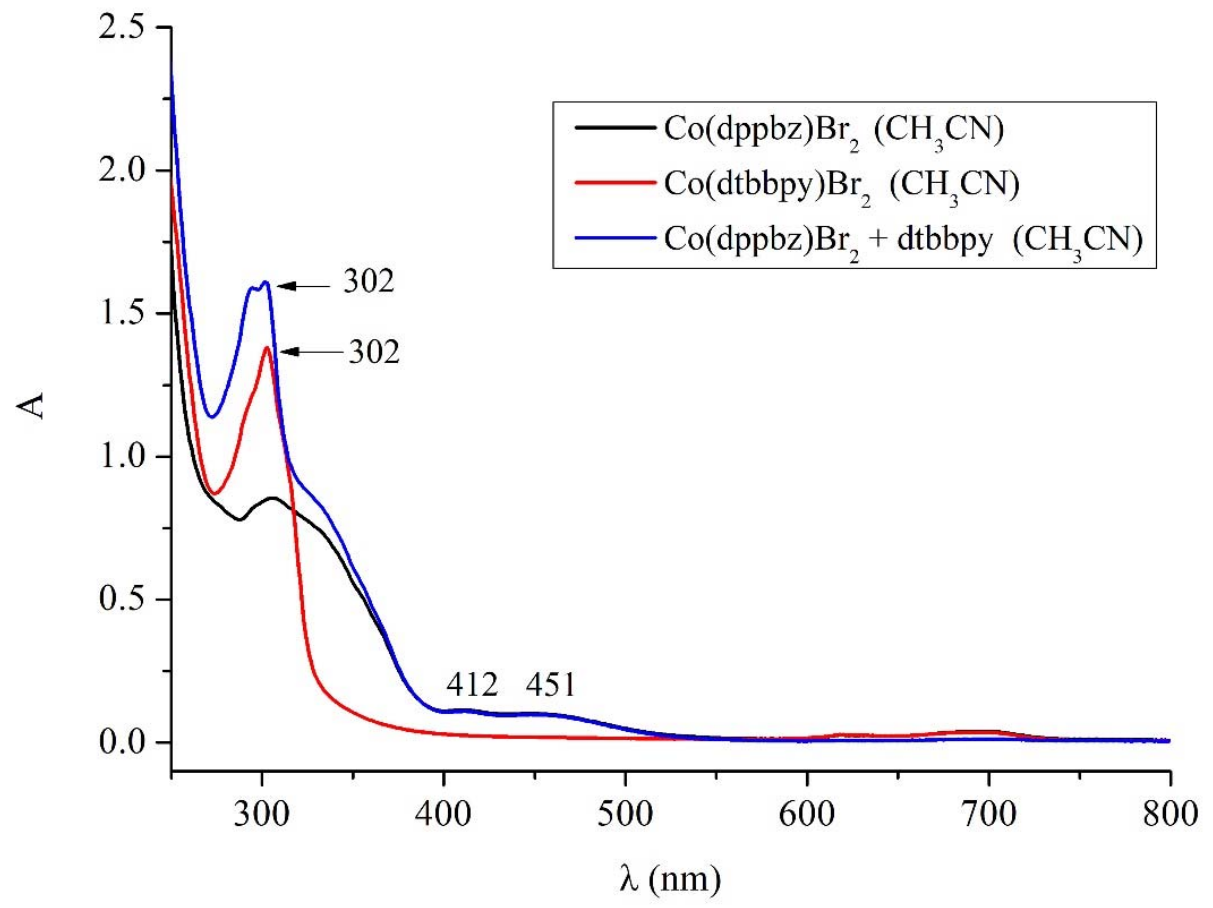

Conditions: $\mathrm{Co}(\mathrm{dppbz}) \mathrm{Br}_{2}(0.01 \mathrm{mmol})$ in $100 \mathrm{~mL} \mathrm{CH} \mathrm{CH}_{3} \mathrm{CN}$ (dark); $\mathrm{Co}$ (dtbbpy) $\mathrm{Br}_{2}(0.01 \mathrm{mmol})$ in $100 \mathrm{~mL} \mathrm{CH}_{3} \mathrm{CN}$ (red); $\mathrm{Co}(\mathrm{dppbz}) \mathrm{Br}_{2}(0.01 \mathrm{mmol})+$ dtbbpy $(0.005 \mathrm{mmol})$ in $\left.100 \mathrm{~mL} \mathrm{CH} \mathrm{CH}_{3} \mathrm{Cblue}\right)$.

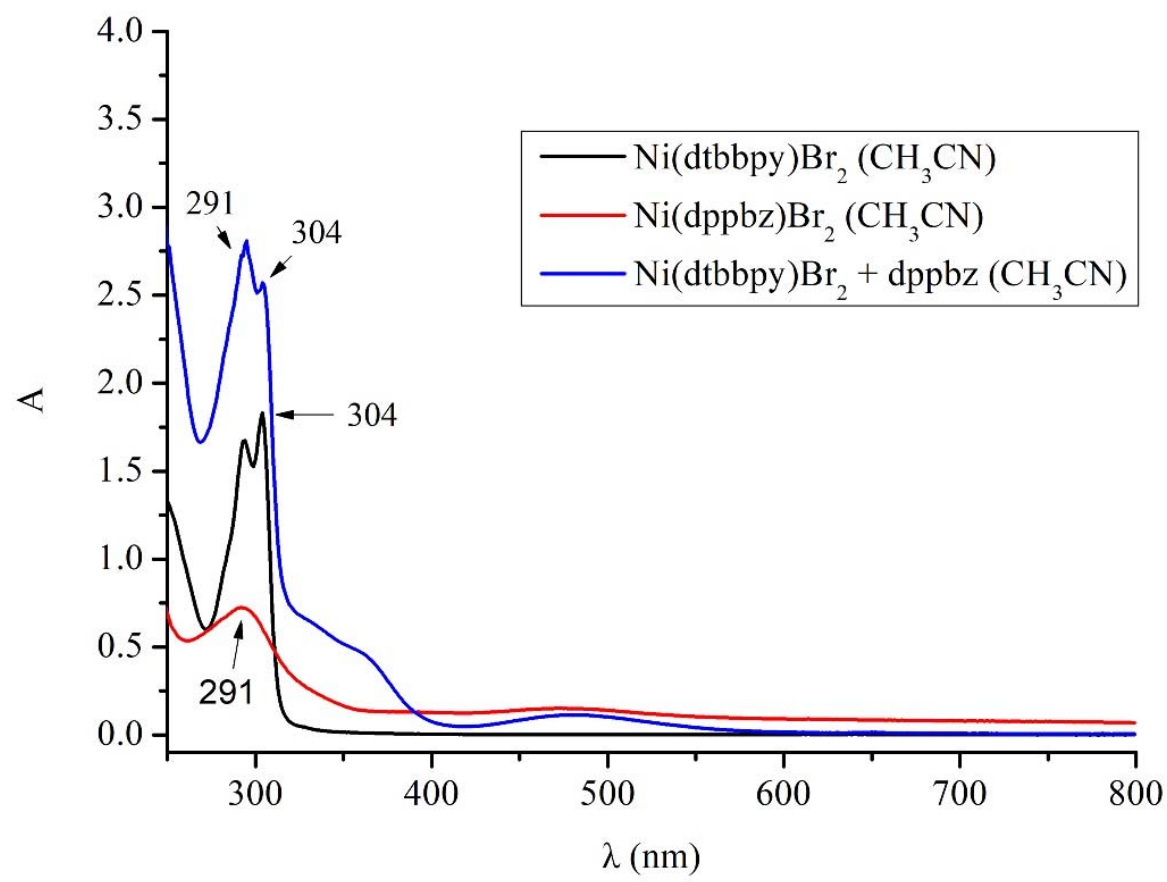

Conditions: $\mathrm{Ni}$ (dtbbpy) $\mathrm{Br}_{2}(0.01 \mathrm{mmol})$ in $100 \mathrm{~mL} \mathrm{CH} \mathrm{CH}_{3} \mathrm{CN}$ (dark); $\mathrm{Ni}(\mathrm{dppbz}) \mathrm{Br}_{2}(0.005 \mathrm{mmol})$ in $100 \mathrm{~mL} \mathrm{CH} 3 \mathrm{CN}$ (red); $\mathrm{Ni}(\mathrm{dtbbpy}) \mathrm{Br}_{2}(0.01 \mathrm{mmol})+\mathrm{dppbz}(0.01 \mathrm{mmol})$ in $100 \mathrm{~mL} \mathrm{CH} \mathrm{CH}_{3} \mathrm{CN}$ (blue). 


\section{Functionalization and application}

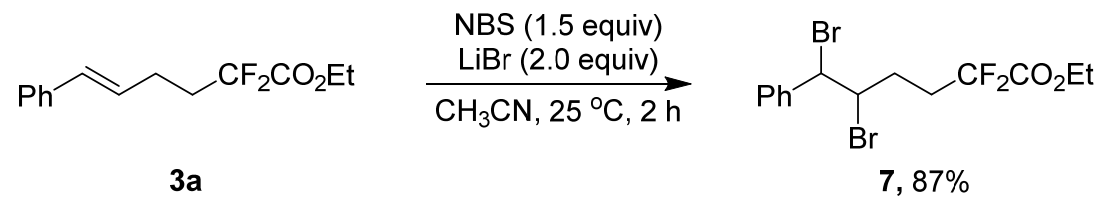

To a solution of 3a $(0.2 \mathrm{mmol})$ in $\mathrm{CH}_{3} \mathrm{CN}(2.0 \mathrm{~mL})$, were added $\mathrm{LiBr}(2.0$ equiv) and $\mathrm{N}$ Bromosuccinimide (NBS, 1.5 equiv) in a vial $(10.0 \mathrm{~mL})$. Then the mixture was stirred at $25^{\circ} \mathrm{C}$ for $2 \mathrm{~h}$. After the reaction finished that monitored by TLC, the reaction mixture was concentrated under vacuum. The residue was purified by flash column chromatography (petroleum ether as eluent) to give the product 7 as a colorless oil $(72.0 \mathrm{mg}, 87 \%$ yield).

${ }^{1} \mathbf{H}$ NMR $\left(500 \mathrm{MHz}, \mathrm{CDCl}_{3}\right) \delta 7.37-7.36(\mathrm{~m}, 4 \mathrm{H}), 7.35-7.32(\mathrm{~m}, 1 \mathrm{H}), 5.03(\mathrm{~d}, J=10.5 \mathrm{~Hz}, 1 \mathrm{H}), 4.55$ $-4.51(\mathrm{~m}, 1 \mathrm{H}), 4.36(\mathrm{q}, J=7.5 \mathrm{~Hz}, 2 \mathrm{H}), 2.75-2.69(\mathrm{~m}, 1 \mathrm{H}), 2.57-2.45(\mathrm{~m}, 1 \mathrm{H}), 2.38-2.29(\mathrm{~m}, 1 \mathrm{H})$, $2.27-2.17(\mathrm{~m}, 1 \mathrm{H}), 1.37(\mathrm{t}, J=7.5 \mathrm{~Hz}, 3 \mathrm{H})$.

${ }^{13} \mathbf{C}$ NMR $\left(151 \mathrm{MHz}, \mathrm{CDCl}_{3}\right) \delta 163.9(\mathrm{t}, J=32.8 \mathrm{~Hz}), 140.0,128.9,128.7,127.7,115.7(\mathrm{t}, J=251.0$ $\mathrm{Hz}), 63.1,56.4,56.1,32.2(\mathrm{t}, J=23.6 \mathrm{~Hz}), 29.6(\mathrm{t}, J=4.5 \mathrm{~Hz}), 14.0$.

${ }^{19}$ F NMR $\left(471 \mathrm{MHz}, \mathrm{CDCl}_{3}\right) \delta-104.9-(-106.6)(\mathrm{m})$.

HRMS (ESI-TOF) (m/z): calcd for $\mathrm{C}_{14} \mathrm{H}_{26} \mathrm{Br}_{2} \mathrm{~F}_{3} \mathrm{NaO}_{2}{ }^{+}\left([\mathrm{M}+\mathrm{Na}]^{+}\right)$, 434.9377; found, 434.9373 .

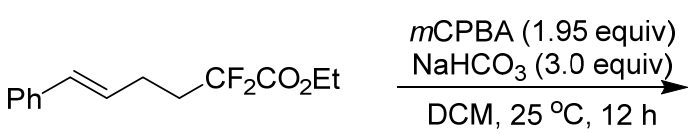

3a<smiles>CCOC(=O)CCCC1OC1c1ccccc1</smiles>

$8,85 \%$

To a solution of $\mathbf{3 a}(0.2 \mathrm{mmol})$ in DCM $(2.0 \mathrm{~mL})$, was added 3-Chloroperoxybenzoic Acid ( $\mathrm{mCPBA}$, $67.3 \mathrm{mg}, 1.95$ equiv) and $\mathrm{NaHCO}_{3}(50.4 \mathrm{mg}, 3.0$ equiv) in a vial $(10.0 \mathrm{~mL})$ with magnetic stir bar. Then the mixture was stirred at $25^{\circ} \mathrm{C}$ for $12 \mathrm{~h}$. After the reaction finished that monitored by TLC, the reaction mixture was cooled to $0{ }^{\circ} \mathrm{C}$ and aqueous $\mathrm{Na}_{2} \mathrm{~S}_{2} \mathrm{O}_{3}(5 \%, 2.0 \mathrm{~mL})$ was added and stirred for $5 \mathrm{~min}$ at $0{ }^{\circ} \mathrm{C}$ and $15 \mathrm{~min}$ at $25{ }^{\circ} \mathrm{C}$. The mixture was washed with equal volumes of saturated aqueous brine, saturated aqueous $\mathrm{NaHCO}_{3}$. The combined aqueous phases were re-extracted with $\operatorname{DCM}(3 \times 5.0 \mathrm{~mL})$ and the combined organic extracts were dried over $\mathrm{Na}_{2} \mathrm{SO}_{4}$, filtered off and the solvent was removed in vacuo, the crude product was purified by column chromatography to give the product 8 as a colorless oil ( $45.9 \mathrm{mg}, 85 \%$ yield). ${ }^{20}$

${ }^{1} \mathbf{H}$ NMR $\left(500 \mathrm{MHz}, \mathrm{CDCl}_{3}\right) \delta 7.36-7.29(\mathrm{~m}, 3 \mathrm{H}), 7.26-7.25(\mathrm{~m}, 2 \mathrm{H}), 4.32(\mathrm{q}, J=7.0 \mathrm{~Hz}, 2 \mathrm{H}), 3.65$ $(\mathrm{d}, J=2.0 \mathrm{~Hz}, 1 \mathrm{H}), 3.02-3.00(\mathrm{~m}, 1 \mathrm{H}), 2.36-2.23(\mathrm{~m}, 2 \mathrm{H}), 2.01-1.94(\mathrm{~m}, 1 \mathrm{H}), 1.89-1.82(\mathrm{~m}, 1 \mathrm{H})$, $1.35(\mathrm{t}, J=7.0 \mathrm{~Hz}, 3 \mathrm{H})$.

${ }^{13} \mathrm{C}$ NMR $\left(151 \mathrm{MHz}, \mathrm{CDCl}_{3}\right) \delta 163.9(\mathrm{t}, J=32.8 \mathrm{~Hz}), 137.0,128.5,128.2,125.5,115.7(\mathrm{t}, J=250.5$ $\mathrm{Hz}), 63.0,61.4,58.7,30.8(\mathrm{t}, J=23.7 \mathrm{~Hz}), 24.6(\mathrm{t}, J=4.1 \mathrm{~Hz}), 13.9$.

${ }^{19} \mathbf{F}$ NMR $\left(471 \mathrm{MHz}, \mathrm{CDCl}_{3}\right) \delta-105.3-(-106.8)(\mathrm{m})$.

HRMS (ESI-TOF) (m/z): calcd for $\mathrm{C}_{14} \mathrm{H}_{16} \mathrm{~F}_{2} \mathrm{NaO}_{3}{ }^{+}\left([\mathrm{M}+\mathrm{Na}]^{+}\right), 293.0960$; found, 293.0963 . 


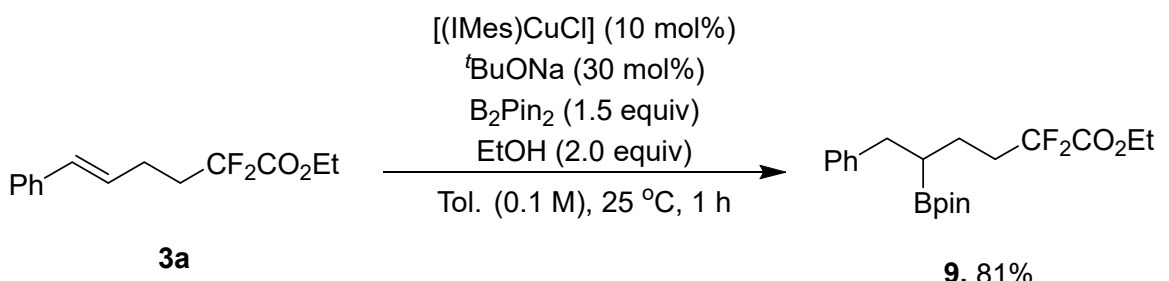

Into a nitrogen-filled glove box, an oven-dried vial $(10.0 \mathrm{~mL})$ with magnetic stir bar was charged with (IMes) $\mathrm{CuCl}(8.0 \mathrm{mg}, 10 \mathrm{~mol} \%), \mathrm{NaO}^{t} \mathrm{Bu}(5.8 \mathrm{mg}, 30 \mathrm{~mol} \%)$, and toluene $(2.0 \mathrm{~mL})$. The mixture was allowed to stir for $5 \mathrm{~min}$. Then $\mathrm{B}_{2} \mathrm{Pin}_{2}(76.0 \mathrm{mg}, 1.5$ equiv) was added to the mixture. After stirred for $10 \mathrm{~min}, 3 \mathbf{3}(0.2 \mathrm{mmol})$ and $\mathrm{EtOH}(24 \mu \mathrm{L}, 2.0$ equiv) were added by syringes. The resulting solution was allowed to stir at $25^{\circ} \mathrm{C}$ for $1 \mathrm{~h}$. After the reaction finished that monitored by TLC, the solution was passed through a short plug of celite and washed with $\mathrm{Et}_{2} \mathrm{O}(10.0 \mathrm{~mL})$. The filtrate was concentrated in vacuo and then purified by silica gel column chromatography to give the product 9 as colorless oil $\left(61.9 \mathrm{mg}, 81 \%\right.$ yield). ${ }^{21}$

${ }^{1} \mathbf{H}$ NMR $\left(500 \mathrm{MHz}, \mathrm{CDCl}_{3}\right) \delta 7.24-7.23(\mathrm{~m}, 2 \mathrm{H}), 7.19-7.14(\mathrm{~m}, 3 \mathrm{H}), 4.28$ (q, J $\left.=7.0 \mathrm{~Hz}, 2 \mathrm{H}\right), 2.78$ $-2.74(\mathrm{~m}, 1 \mathrm{H}), 2.66-2.62(\mathrm{~m}, 1 \mathrm{H}), 2.18-2.02(\mathrm{~m}, 2 \mathrm{H}), 1.58-1.53(\mathrm{~m}, 2 \mathrm{H}), 1.38-1.35(\mathrm{~m}, 1 \mathrm{H}), 1.31$ (t, $J=7.5 \mathrm{~Hz}, 3 \mathrm{H}), 1.17$ (s, 6H), $1.15(\mathrm{~s}, 6 \mathrm{H})$.

${ }^{13}$ C NMR $\left(151 \mathrm{MHz}, \mathrm{CDCl}_{3}\right) \delta 164.3(\mathrm{t}, J=33.1 \mathrm{~Hz}), 141.4,128.8,128.1,125.8,116.3(\mathrm{t}, J=249.8$ $\mathrm{Hz}), 83.3,62.6,36.8,34.9(\mathrm{t}, J=23.1 \mathrm{~Hz}), 24.8,24.7,22.5(\mathrm{t}, J=4.2 \mathrm{~Hz}), 13.9$.

${ }^{19} \mathbf{F}$ NMR (471 MHz, $\left.\mathrm{CDCl}_{3}\right) \delta-105.8-(-105.9)(\mathrm{m})$.

${ }^{11}$ B NMR $\left(193 \mathrm{MHz}, \mathrm{CDCl}_{3}\right) \delta 33.9$.

HRMS (ESI-TOF) (m/z): calcd for $\mathrm{C}_{20} \mathrm{H}_{29} \mathrm{BF}_{2} \mathrm{NaO}_{4}{ }^{+}\left([\mathrm{M}+\mathrm{Na}]^{+}\right)$, 405.2023; found, 425.2024.

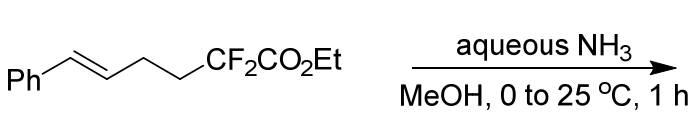

$3 \mathbf{a}$<smiles>NC(=O)C(F)(F)CC/C=C/c1ccccc1</smiles>

$10,71 \%$

To a $25 \mathrm{~mL}$ round-bottom flask were added compound $\mathbf{3 a}(0.2 \mathrm{mmol})$ and $2.0 \mathrm{~mL} \mathrm{MeOH}$ under air. Under stirring, the solution was cooled to $0{ }^{\circ} \mathrm{C}$ and treated with $2.0 \mathrm{~mL} 25 \%$ aqueous ammonia dropwise. The reaction was warmed to $25^{\circ} \mathrm{C}$ and monitored by TLC. After $1 \mathrm{~h}$ or complete consumption of $\mathbf{3 a}, \mathrm{MeOH}$ was evaporated and the aqueous phase was extracted with EtOAc. The organic phase was washed with brine, dried with $\mathrm{Na}_{2} \mathrm{SO}_{4}$ and concentrated. The residue was purified by silica gel chromatography to give the product 10 as a white solid (32.0 mg, 71\% yield), Mp: $89-91{ }^{\circ} \mathrm{C} .{ }^{22}$

${ }^{1}$ H NMR $\left(500 \mathrm{MHz}, \mathrm{CDCl}_{3}\right) \delta 7.34-7.32(\mathrm{~m}, 2 \mathrm{H}), 7.31-7.28(\mathrm{~m}, 2 \mathrm{H}), 7.23-7.10(\mathrm{~m}, 1 \mathrm{H}), 6.44(\mathrm{~d}, J$ $=15.5 \mathrm{~Hz}, 1 \mathrm{H}), 6.27(\mathrm{~s}, 1 \mathrm{H}), 6.20-6.14(\mathrm{~m}, 1 \mathrm{H}), 5.86(\mathrm{~s}, 1 \mathrm{H}), 2.45-2.41(\mathrm{~m}, 2 \mathrm{H}), 2.35-2.25(\mathrm{~m}, 2 \mathrm{H})$. ${ }^{13}$ C NMR $\left(151 \mathrm{MHz}, \mathrm{CDCl}_{3}\right) \delta 166.2(\mathrm{t}, J=29.8 \mathrm{~Hz}), 137.2,131.3,128.5,127.6,127.3,126.0,117.7$ (t, $J=252.8 \mathrm{~Hz}), 33.4(\mathrm{t}, J=23.1 \mathrm{~Hz}), 25.2(\mathrm{t}, J=4.8 \mathrm{~Hz})$.

${ }^{19} \mathbf{F}$ NMR $\left(565 \mathrm{MHz}, \mathrm{CDCl}_{3}\right) \delta-105.8(\mathrm{t}, J=17.5 \mathrm{~Hz})$.

HRMS (ESI-TOF) $(\mathrm{m} / \mathrm{z})$ : calcd for $\mathrm{C}_{12} \mathrm{H}_{13} \mathrm{~F}_{2} \mathrm{NNaO}^{+}\left([\mathrm{M}+\mathrm{Na}]^{+}\right)$, 248.0857; found, 248.0860 . 


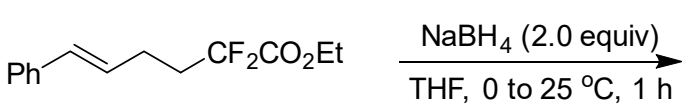

$3 a$<smiles>OCC(F)(F)CC/C=C/c1ccccc1</smiles>

$11,86 \%$

To a $25 \mathrm{~mL}$ round-bottom flask were added compound $\mathbf{3 a}(0.2 \mathrm{mmol})$ and $2.0 \mathrm{~mL}$ THF under air. Under stirring, the solution was cooled to $0{ }^{\circ} \mathrm{C}$, then $\mathrm{NaBH}_{4}\left(15.1 \mathrm{mg}, 2.0\right.$ equiv) was added at $0{ }^{\circ} \mathrm{C}$. The reaction was warmed to $25{ }^{\circ} \mathrm{C}$ and monitored by TLC. After $1 \mathrm{~h}, \mathrm{H}_{2} \mathrm{O}$ was added, extracted with EtOAc, The organic phase was washed with brine, dried with $\mathrm{Na}_{2} \mathrm{SO}_{4}$ and concentrated. The residue was purified by silica gel chromatography to give the product 11 as a colorless oil (36.5 mg, 86\% yield). ${ }^{23}$

${ }^{1} \mathbf{H}$ NMR $\left(600 \mathrm{MHz}, \mathrm{CDCl}_{3}\right) \delta 7.34(\mathrm{~d}, J=7.2 \mathrm{~Hz}, 2 \mathrm{H}), 7.30(\mathrm{t}, J=7.8 \mathrm{~Hz}, 2 \mathrm{H}), 7.21(\mathrm{t}, J=7.2 \mathrm{~Hz}$, $1 \mathrm{H}), 6.45(\mathrm{~d}, J=15.6 \mathrm{~Hz}, 1 \mathrm{H}), 6.23-6.18(\mathrm{~m}, 1 \mathrm{H}), 3.80-3.75(\mathrm{~m}, 2 \mathrm{H}), 2.46-2.42(\mathrm{~m}, 2 \mathrm{H}), 2.15-$ $2.07(\mathrm{~m}, 2 \mathrm{H}), 1.84(\mathrm{t}, J=7.2 \mathrm{~Hz}, 1 \mathrm{H})$.

${ }^{13} \mathbf{C}$ NMR $\left(151 \mathrm{MHz}, \mathrm{CDCl}_{3}\right) \delta 137.3,130.9,128.53,128.46,127.2,126.0,122.9(\mathrm{t}, J=242.2 \mathrm{~Hz}), 64.2$ $(\mathrm{t}, J=32.2 \mathrm{~Hz}), 33.0(\mathrm{t}, J=23.9 \mathrm{~Hz}), 25.3(\mathrm{t}, J=5.0 \mathrm{~Hz})$.

${ }^{19}$ F NMR $\left(565 \mathrm{MHz}, \mathrm{CDCl}_{3}\right) \delta-108.56-(-108.66)(\mathrm{m})$.

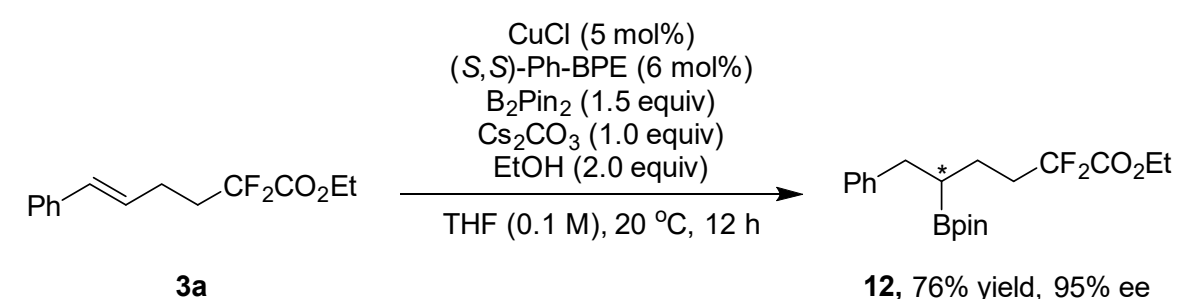

Into a nitrogen-filled glove box, an oven-dried vial $(10.0 \mathrm{~mL})$ with magnetic stir bar was charged with $\mathrm{CuCl}(1.0 \mathrm{mg}, 5 \mathrm{~mol} \%)$, (S,S)-Ph-BPE (6.0 mg, $6 \mathrm{~mol} \%$ ), and THF (2.0 mL). Then $\mathrm{Cs}_{2} \mathrm{CO}_{3}$ (65.2 $\mathrm{mg}, 1.0$ equiv) was added, the mixture was allowed to stir for $5 \mathrm{~min}$. Then $\mathrm{B}_{2} \mathrm{Pin}_{2}(76.0 \mathrm{mg}$, 1.5 equiv) was added to the mixture. After stirred for $10 \mathrm{~min}, \mathbf{3 a}(0.2 \mathrm{mmol})$ and $\mathrm{EtOH}(24 \mu \mathrm{L}, 2.0$ equiv) were added by syringes. The resulting solution was allowed to stir at $20^{\circ} \mathrm{C}$ for $12 \mathrm{~h}$. After the reaction finished that monitored by TLC, the solution was passed through a short plug of celite and washed with $\mathrm{Et}_{2} \mathrm{O}(10.0 \mathrm{~mL})$. The filtrate was concentrated in vacuo and then purified by silica gel column chromatography to give the product 12 as colorless oil (58.0 mg, 76\% yield, 95\% ee). Specific rotation $[\alpha]_{\mathrm{D}}{ }^{25}=-36.6,\left(c=1.00, \mathrm{CHCl}_{3}\right)$. HPLC analysis (OD-H, Hexane/IPA $=98 / 2,1.0$ $\mathrm{mL} / \mathrm{min}, 210 \mathrm{~nm}$ ) indicated $95 \%$ ee: $t_{\mathrm{R}}($ minor $)=5.626 \mathrm{~min}, t_{\mathrm{R}}($ maior $)=6.889 \mathrm{~min}$.
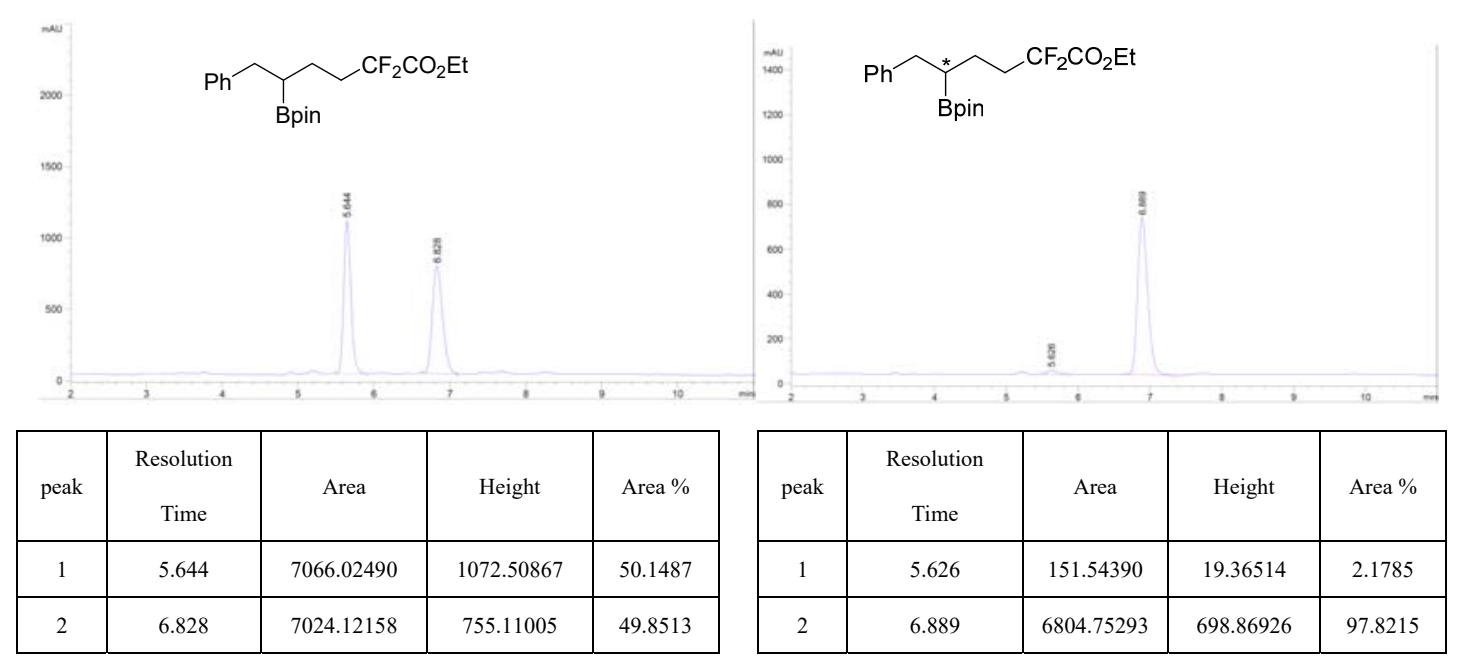


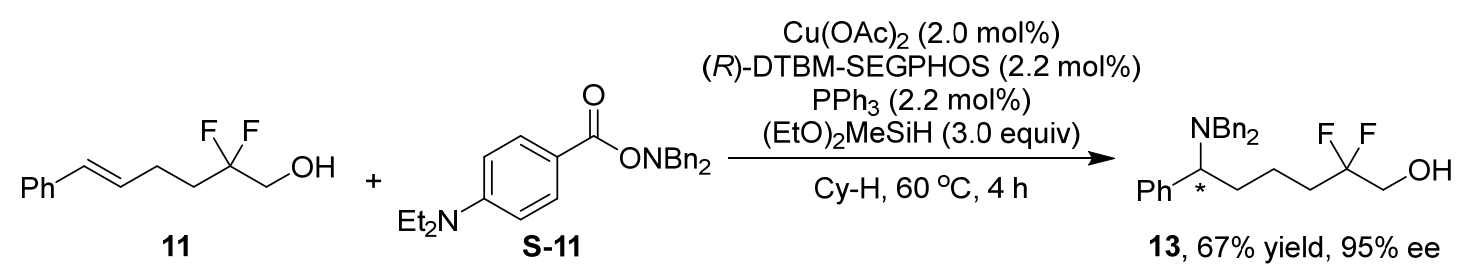

Into a nitrogen-filled glove box, to a screw-cap oven-dried reaction tube equipped with a stir bar was added $11(0.2 \mathrm{mmol})$ and hydroxylamine ester (S-11, $85.3 \mathrm{mg}, 1.1$ equiv). In the glove box, a separate oven-dried reaction tube (II) equipped with a stir bar was charged with $\mathrm{Cu}(\mathrm{OAc})_{2}(0.7 \mathrm{mg}, 2.0 \mathrm{~mol} \%)$, (R)-DTBM-SEGPHOS (5.6 mg, $2.2 \mathrm{~mol} \%), \mathrm{PPh}_{3}(1.1 \mathrm{mg}, 2.2 \mathrm{mmol} \%$ ), Cyclohexane $(0.5 \mathrm{~mL})$ was added to reaction tube II via a syringe and the mixture was stirred at $25^{\circ} \mathrm{C}$ for $5 \mathrm{~min}$ until a homogenous blue solution formed, then diethoxymethylsilane $\left(\left(\mathrm{Et}_{2} \mathrm{O}\right)_{2} \mathrm{MeSiH}, 96 \mu \mathrm{L}, 3.0\right.$ equiv) was added via syringe. The resulting mixture was allowed to stir at $25^{\circ} \mathrm{C}$ for an additional $10 \mathrm{~min}$, and the solution changed colors from blue to bright yellow. To reaction tube I was added cyclohexane $(0.5 \mathrm{~mL})$ and then the $\mathrm{CuH}$ catalyst solution from reaction tube II via syringe. Reaction tube I was capped, removed from the glove box, and the reaction mixture was stirred at $60^{\circ} \mathrm{C}$ for $4 \mathrm{~h}$. After the reaction finished that monitored by TLC, the reaction mixture was cooled to $25^{\circ} \mathrm{C}$, and was treated with saturated $\mathrm{NH}_{4} \mathrm{~F}$ solution (in $\mathrm{MeOH})(2.0 \mathrm{~mL})$, and stirred at $25^{\circ} \mathrm{C}$ for $30 \mathrm{~min}$. Aqueous saturated $\mathrm{NaHCO}_{3}$ solution $(2.0$ $\mathrm{mL}$ ) was then added and the mixture was stirred at $25{ }^{\circ} \mathrm{C}$ for an additional $30 \mathrm{~min}$, followed by addition of a saturated aqueous solution of $\mathrm{NaHCO}_{3}(10.0 \mathrm{~mL})$ and EtOAc $(10.0 \mathrm{~mL})$. The phases were separated and the aqueous layer was extracted with EtOAc $(2 \times 10.0 \mathrm{~mL})$. The combined organic phases were washed with brine $(20.0 \mathrm{~mL})$, dried over $\mathrm{Na}_{2} \mathrm{SO}_{4}$, and concentrated in vacuo, the residue was purified by silica gel chromatography to give the product 13 as a colorless oil (54.8 mg, 67\% yield, $95 \%$ ee) ${ }^{24}$

${ }^{1} \mathbf{H}$ NMR $\left(600 \mathrm{MHz}, \mathrm{CDCl}_{3}\right) \delta 7.39-7.37(\mathrm{~m}, 5 \mathrm{H}), 7.33-7.30(\mathrm{~m}, 6 \mathrm{H}), 7.24-7.20(\mathrm{~m}, 4 \mathrm{H}), 3.82(\mathrm{~d}, J$ $=13.8 \mathrm{~Hz}, 2 \mathrm{H}), 3.69-3.63(\mathrm{~m}, 3 \mathrm{H}), 3.12(\mathrm{~d}, J=13.8 \mathrm{~Hz}, 2 \mathrm{H}), 2.16-2.10(\mathrm{~m}, 1 \mathrm{H}), 1.86-1.74(\mathrm{~m}, 4 \mathrm{H})$, $1.72-1.62(\mathrm{~m}, 1 \mathrm{H}), 1.53-1.45(\mathrm{~m}, 1 \mathrm{H})$.

${ }^{13} \mathbf{C}$ NMR $\left(151 \mathrm{MHz}, \mathrm{CDCl}_{3}\right) \delta 140.2,138.4,128.9,128.8,128.2,128.0,127.2,126.8,123.2(\mathrm{t}, J=241.9$ $\mathrm{Hz}), 64.1(\mathrm{t}, J=31.9 \mathrm{~Hz}), 61.5,53.7,33.0(\mathrm{t}, J=23.9 \mathrm{~Hz}), 31.2,19.1(\mathrm{t}, J=4.5 \mathrm{~Hz})$.

${ }^{19} \mathbf{F}$ NMR $\left(565 \mathrm{MHz}, \mathrm{CDCl}_{3}\right) \delta-108.54-(-108.65)(\mathrm{m})$.

HRMS (ESI-TOF) (m/z): calcd for $\mathrm{C}_{26} \mathrm{H}_{30} \mathrm{~F}_{2} \mathrm{NO}^{+}\left([\mathrm{M}+\mathrm{Na}]^{+}\right)$, 410.2290 ; found, 410.2296 .

Specific rotation $[\alpha]_{\mathrm{D}}{ }^{25}=-87.5,\left(c=1.00, \mathrm{CHCl}_{3}\right)$. HPLC analysis $(\mathrm{IC}$, Hexane/IPA $=96 / 4,1.0$ $\mathrm{mL} / \mathrm{min}, 254 \mathrm{~nm}$ ) indicated $95 \%$ ee: $t_{\mathrm{R}}($ maior $)=8.962 \mathrm{~min}, t_{\mathrm{R}}($ minor $)=10.575 \mathrm{~min}$.

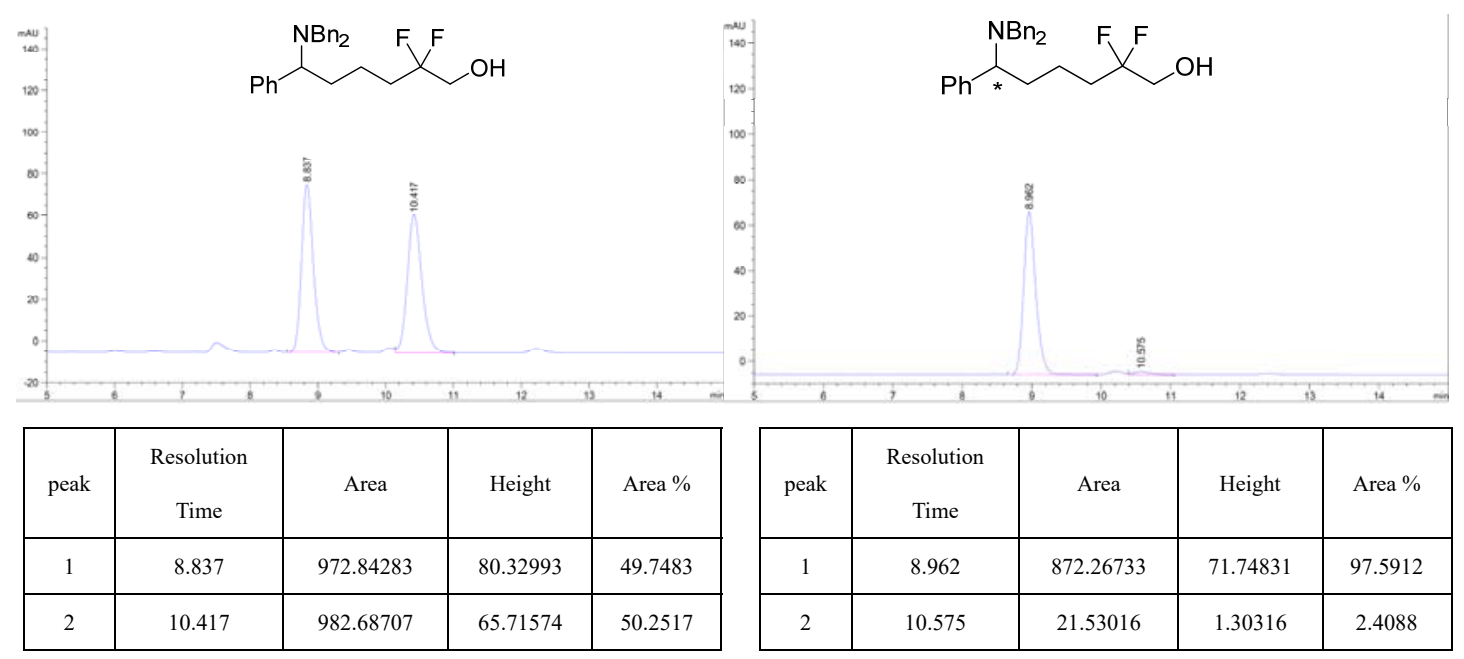




\section{References}

(1) Anka-Lufford, L. L.; Huihui, K. M. M.; Gower, N. J.; Ackerman, L. K. G. ; Weix, D. J. NickelCatalyzed Cross-Electrophile Coupling with Organic Reductants in Non-Amide Solvents. Chem. Eur. J. 2016, 22, 11564-11567.

(2) Grutters, M. M. P.; Vlugt, J. I.; Pei, Y.; Mills, A. M.; Lutz, M.; Spek, A. L.; Mller, C., Moberg, C.; Vogt, D. Highly Selective Cobalt-Catalyzed Hydrovinylation of Styrene. Adv. Synth. Catal. 2009, 351, 2199-2208.

(3) Okuyama, T.; Tani, Y.; Miyake, K.; Yokoyama, Y. Chiral Helicenoid Diarylethene with Large Change in Specific Optical Rotation by Photochromism. J. Org. Chem. 2007, 72, 1634-1638.

(4) Doni, E.; Mondal, B.; O'Sullivan, S.; Tuttle, T.; Murphy, J. A. Overturning Established Chemoselectivities: Selective Reduction of Arenes over Malonates and Cyanoacetates by Photoactivated Organic Electron Donors. J. Am. Chem. Soc. 2013, 135, 10934-10937.

(5) Meng, Q.-Y.; Schirmer, T. E.; Katou, K.; König, B. Controllable Isomerization of Alkenes by Dual Visible-Light-Cobalt Catalysis. Angew. Chem. Int. Ed. 2019, 58, 5723-5728.

(6) Kanehara, M.; Oumi, Y.; Sano, T.; Teranishi, T. Syntheses of the Novel Acidic and Basic Ligands and Superlattice Formation from Gold Nanoparticles through Interparticle Acid-Base Interaction. Bull. Chem. Soc. Jpn. 2004, 77, 1589-1597.

(7) Gladfelder, J. J.; Ghosh, S.; Podunavac, M.; Cook, A. W.; Ma, Y.; Woltornist, R. A.; Keresztes, I.; Hayton, T. W.; Collum, D. B.; Zakarian, A. Enantioselective Alkylation of 2-Alkylpyridines Controlled by Organolithium Aggregation. J. Am. Chem. Soc. 2019, 141, 15024-15028.

(8) Lin, S.; Song, C.-X.; Cai, G.-X.; Wang, W.-H.; Shi, Z.-J. Intra/Intermolecular Direct Allylic Alkylation via Pd(II)-Catalyzed Allylic C-H Activation. J. Am. Chem. Soc. 2008, 130, 12901-12903.

(9) Kapat, A.; Sperger, T.; Guven, S.; Schoenebeck, F. E-Olefins through intramolecular radical relocation. Science. 2019, 363, 391-396.

(10) Zhang, Y.; Torker, S.; Sigrist, M.; Bregović, N.; Dydio, P. Pd(I)-Pd(I) Catalysis Assisted by Iodide Ligands for Selective Hydroformylation of Alkenes and Alkynes. J. Am. Chem. Soc. 2020, 142, 18251-18265.

(11) Dobah, F.; Mazodze, C. M.; Petersen, W. F. Cross-Dehydrogenative Cyclization-Dimerization Cascade Sequence for the Synthesis of Symmetrical 3,3'-Bisoxindoles. Org. Lett. 2021, 23, 54665470.

(12) Benedetto, E.; Keita, M.; Tredwell, M.; Hollingworth, C.; Brown, J. M.; Gouverneur, V. PlatinumCatalyzed Substitution of Allylic Fluorides. Organometallics. 2012, 31, 1408-1416.

(13) Uno, M.; Sumino, S.; Fukuyama, T.; Matsuura, M.; Kuroki, Y.; Kishikawa, Y.; Ryu, I. Synthesis of 4,4-Difluoroalkenes by Coupling of $\alpha$-Substituted $\alpha, \alpha$-Difluoromethyl Halides with Allyl Sulfones under Photoredox Catalyzed Conditions. J. Org. Chem. 2019, 84, 9330-9338.

(14) Wang, L.; Wei, X.-J.; Jia, W.-L.; Zhong, J.-J.; Wu, L.-Z.; Liu, Q. Visible-Light-Driven Difluoroacetamidation of Unactive Arenes and Heteroarenes by Direct C-H Functionalization at Room Temperature. Org. Lett. 2014, 16, 5842-5845.

(15) Yin, Z.-B.; Ye, J.-H.; Zhou, W.-J.; Zhang, Y.-H.; Ding, L.; Gui, Y.-Y.; Yan, S.-S.; Li, J.; Yu, D.-G. Oxy-Difluoroalkylation of Allylamines with $\mathrm{CO}_{2}$ via Visible-Light Photoredox Catalysis. Org. Lett. 2018, 20, 190-193.

(16) Li, D.; Mao, T.; Huang, J.; Zhu, Q. Copper-Catalyzed Bromodifluoroacetylation of Alkenes with Ethyl Bromodifluoroacetate. J. Org. Chem. 2018, 83, 10445-10452.

(17) Shaw, M. H.; Croft, R. A.; Whittingham, W. G.; Bower, J. F. Modular Access to Substituted 
Azocanes via a Rhodium-Catalyzed Cycloaddition-Fragmentation Strategy. J. Am. Chem. Soc. 2015, $137,8054-8057$.

(18) Kohler, D. G.; Gockel, S. N.; Kennemur, J. L.; Waller, P. J.; Hull, K. L. Palladium-catalysed antiMarkovnikov selective oxidative amination. Nat. Chem. 2018, 10, 333-340.

(19) (a) Riente, P.; Pericàs, M. A. Visible Light-Driven Atom Transfer Radical Addition to Olefins using $\mathrm{Bi}_{2} \mathrm{O}_{3}$ as Photocatalyst. ChemSusChem 2015, 8, 1841-1844. (b) Li X.; He S.; Song Q. DiethylzincMediated Radical 1,2-Addition of Alkenes and Alkynes. Org. Lett. 2021, 23, 2994-2999

(20) Bojaryn, K.; Fritsch, S.; Hirschhauser, C. Iterative Synthesis of Alkenes by Insertion of Lithiated Epoxides into Boronic Esters. Org. Lett. 2019, 21, 2218-2222.

(21) Lee, Y.; Hoveyda, A. H. Efficient Boron-Copper Additions to Aryl-Substituted Alkenes Promoted by NHC-Based Catalysts. Enantioselective Cu-Catalyzed Hydroboration Reactions. J. Am. Chem. Soc. 2009, 131, 3160-3161.

(22) Xu, C.; Yang, Z.-F.; An, L.; Zhang, X. Nickel-Catalyzed Difluoroalkylation-Alkylation of Enamides. ACS Catal. 2019, 9, 8224-8229.

(23) Tian, P.-P.; Xiao, H.-Q.; Wang, L.; Yu, Y.; Huang, Y. Nucleophilic fluoroalkylation of terminal vinyl triflones with $\mathrm{SO}_{2} \mathrm{CF}_{3}$ as a removable activating group. Tetrahdron Lett. 2019, 60, 1015-1018.

(24) Ichikawa, S.; Buchwald, S. L. Asymmetric Synthesis of $\gamma$-Amino Alcohols by Copper-Catalyzed Hydroamination. Org. Lett. 2019, 21, 8736-8739. 


\section{XI. ${ }^{1} \mathrm{H},{ }^{13} \mathrm{C},{ }^{19} \mathrm{~F},{ }^{11} \mathrm{~B}$ NMR Spectra of New Compounds}<smiles>CCOC(=O)CCCC=Cc1ccccc1</smiles>

\section{ethyl (E)-2,2-difluoro-6-phenylhex-5-enoate (3a)}

Following the General Procedure at a $0.2 \mathrm{mmol}$ scale with alkene to afford the tittle compound $\mathbf{3 a}$ as a colorless oil with $72 \%$ yield $(36.6 \mathrm{mg})$.

${ }^{1} \mathbf{H}$ NMR (600 MHz, $\left.\mathrm{CDCl}_{3}\right) \delta 7.33(\mathrm{~d}, J=7.2 \mathrm{~Hz}, 2 \mathrm{H}), 7.30(\mathrm{t}, J=7.2 \mathrm{~Hz}, 2 \mathrm{H}), 7.21(\mathrm{t}, J=7.2 \mathrm{~Hz}, 1 \mathrm{H})$, $6.43(\mathrm{~d}, J=15.6 \mathrm{~Hz}, 1 \mathrm{H}), 6.18-6.13(\mathrm{~m}, 1 \mathrm{H}), 4.29(\mathrm{q}, J=7.2 \mathrm{~Hz}, 2 \mathrm{H}), 2.44-2.40(\mathrm{~m}, 2 \mathrm{H}), 2.30-2.22$ $(\mathrm{m}, 2 \mathrm{H}), 1.33(\mathrm{t}, J=7.2 \mathrm{~Hz}, 3 \mathrm{H})$.

${ }^{13} \mathbf{C}$ NMR $\left(151 \mathrm{MHz}, \mathrm{CDCl}_{3}\right) \delta 164.2(\mathrm{t}, J=32.8 \mathrm{~Hz}), 137.1,131.4,128.5,127.4,127.3,126.0,115.9(\mathrm{t}$, $J=250.7 \mathrm{~Hz}), 62.9,34.3(\mathrm{t}, J=23.1 \mathrm{~Hz}), 25.1(\mathrm{t}, J=5.0 \mathrm{~Hz}), 13.9$.

${ }^{19}$ F NMR $\left(471 \mathrm{MHz}, \mathrm{CDCl}_{3}\right) \delta-106.0(\mathrm{t}, J=16.5 \mathrm{~Hz})$.

HRMS (ESI-TOF) (m/z): calcd for $\mathrm{C}_{14} \mathrm{H}_{16} \mathrm{~F}_{2} \mathrm{NaO}_{2}{ }^{+}\left([\mathrm{M}+\mathrm{Na}]^{+}\right), 277.1011$; found, 277.1015 .<smiles>CCOC(=O)C(F)(F)CC/C=C/c1ccc(C)cc1</smiles>

\section{ethyl (E)-2,2-difluoro-6-(p-tolyl)hex-5-enoate (3b)}

Following the General Procedure at a $0.2 \mathrm{mmol}$ scale with alkene to afford the tittle compound $\mathbf{3 b}$ as a colorless oil with $70 \%$ yield $(37.5 \mathrm{mg})$.

${ }^{1} \mathbf{H}$ NMR $\left(600 \mathrm{MHz}, \mathrm{CDCl}_{3}\right) \delta 7.22(\mathrm{~d}, J=7.8 \mathrm{~Hz}, 2 \mathrm{H}), 7.10(\mathrm{~d}, J=7.8 \mathrm{~Hz}, 2 \mathrm{H}), 6.40(\mathrm{~d}, J=15.6 \mathrm{~Hz}$, $1 \mathrm{H}), 6.11-6.07(\mathrm{~m}, 1 \mathrm{H}), 4.29(\mathrm{q}, J=7.2 \mathrm{~Hz}, 2 \mathrm{H}), 2.42-2.38(\mathrm{~m}, 2 \mathrm{H}), 2.32(\mathrm{~s}, 3 \mathrm{H}), 2.29-2.22(\mathrm{~m}$, 2H), $1.33(\mathrm{t}, J=13.2 \mathrm{~Hz}, 3 \mathrm{H})$.

${ }^{13}$ C NMR $\left(126 \mathrm{MHz}, \mathrm{CDCl}_{3}\right) \delta 164.2(\mathrm{t}, J=33.4 \mathrm{~Hz}), 137.1,134.3,131.2,129.2,126.3,125.9,115.9(\mathrm{t}$, $J=251.1 \mathrm{~Hz}), 62.8,34.3(\mathrm{t}, J=23.7 \mathrm{~Hz}), 25.1(\mathrm{t}, J=4.8 \mathrm{~Hz}), 21.1,13.9$.

${ }^{19}$ F NMR $\left(471 \mathrm{MHz}, \mathrm{CDCl}_{3}\right) \delta-106.0(\mathrm{t}, J=16.0 \mathrm{~Hz})$.

HRMS (ESI-TOF) (m/z): calcd for $\mathrm{C}_{15} \mathrm{H}_{18} \mathrm{~F}_{2} \mathrm{NaO}_{2}{ }^{+}\left([\mathrm{M}+\mathrm{Na}]^{+}\right), 291.1167$; found, 291.1166 .<smiles>CCOC(=O)C(F)(F)CCC=Cc1cccc(C)c1</smiles>

\section{ethyl (E)-2,2-difluoro-6-(m-tolyl)hex-5-enoate (3c)}

Following the General Procedure at a $0.2 \mathrm{mmol}$ scale with alkene to afford the tittle compound 3c as a colorless oil with $75 \%$ yield $(40.2 \mathrm{mg})$.

${ }^{1} \mathbf{H}$ NMR $\left(600 \mathrm{MHz}, \mathrm{CDCl}_{3}\right) \delta 7.18(\mathrm{t}, J=7.8 \mathrm{~Hz}, 1 \mathrm{H}), 7.15-7.12(\mathrm{~m}, 2 \mathrm{H}), 7.03(\mathrm{~d}, J=7.8 \mathrm{~Hz}, 1 \mathrm{H})$, $6.40(\mathrm{~d}, J=15.6 \mathrm{~Hz}, 1 \mathrm{H}), 6.16-6.11(\mathrm{~m}, 1 \mathrm{H}), 4.29(\mathrm{q}, J=7.2 \mathrm{~Hz}, 2 \mathrm{H}), 2.42-2.39(\mathrm{~m}, 2 \mathrm{H}), 2.33(\mathrm{~s}$, $3 \mathrm{H}), 2.29-2.21(\mathrm{~m}, 2 \mathrm{H}), 1.33(\mathrm{t}, J=7.2 \mathrm{~Hz}, 3 \mathrm{H})$.

${ }^{13}$ C NMR $\left(151 \mathrm{MHz}, \mathrm{CDCl}_{3}\right) \delta 164.2(\mathrm{t}, J=32.3 \mathrm{~Hz}), 138.1,137.1,131.5,128.4,128.1,127.2,126.8$, 123.2, $115.9(\mathrm{t}, J=250.7 \mathrm{~Hz}), 62.8,34.3(\mathrm{t}, J=23.1 \mathrm{~Hz}), 25.1(\mathrm{t}, J=4.8 \mathrm{~Hz}), 21.4,13.9$.

${ }^{19}$ F NMR $\left(471 \mathrm{MHz}, \mathrm{CDCl}_{3}\right) \delta-106.0(\mathrm{t}, J=15.5 \mathrm{~Hz})$.

HRMS (ESI-TOF) (m/z): calcd for $\mathrm{C}_{15} \mathrm{H}_{18} \mathrm{~F}_{2} \mathrm{NaO}_{2}{ }^{+}\left([\mathrm{M}+\mathrm{Na}]^{+}\right), 291.1167$; found, 291.1171 . 
<smiles>CCOC(=O)CCC/C=C/c1ccccc1C</smiles>

\section{ethyl (E)-2,2-difluoro-6-(o-tolyl)hex-5-enoate (3d)}

Following the General Procedure at a $0.2 \mathrm{mmol}$ scale with alkene to afford the tittle compound $\mathbf{3 d}$ as a colorless oil with $60 \%$ yield $(32.1 \mathrm{mg})$.

${ }^{1} \mathrm{H} \mathrm{NMR}\left(600 \mathrm{MHz}, \mathrm{CDCl}_{3}\right) \delta 7.39-7.37(\mathrm{~m}, 1 \mathrm{H}), 7.16-7.12(\mathrm{~m}, 3 \mathrm{H}), 6.64(\mathrm{~d}, J=15.6 \mathrm{~Hz}, 1 \mathrm{H}), 6.05$ $-6.00(\mathrm{~m}, 1 \mathrm{H}), 4.30(\mathrm{q}, J=7.2 \mathrm{~Hz}, 2 \mathrm{H}), 2.46-2.42(\mathrm{~m}, 2 \mathrm{H}), 2.33(\mathrm{~s}, 3 \mathrm{H}), 2.31-2.22(\mathrm{~m}, 2 \mathrm{H}), 1.34(\mathrm{t}$, $J=7.2 \mathrm{~Hz}, 3 \mathrm{H})$.

${ }^{13} \mathrm{C}$ NMR $\left(151 \mathrm{MHz}, \mathrm{CDCl}_{3}\right) \delta 164.2(\mathrm{t}, J=32.0 \mathrm{~Hz}), 136.2,135.1,130.2,129.3,128.8,127.2,126.0$, 125.5, 115.9 (t, $J=250.7 \mathrm{~Hz}), 62.8,34.4(\mathrm{t}, J=23.4 \mathrm{~Hz}), 25.4(\mathrm{t}, J=4.7 \mathrm{~Hz}), 19.8,13.9$.

${ }^{19} \mathrm{~F}$ NMR $\left(565 \mathrm{MHz}, \mathrm{CDCl}_{3}\right) \delta-106.0(\mathrm{t}, J=16.4 \mathrm{~Hz})$.

HRMS (ESI-TOF) $(\mathrm{m} / \mathrm{z})$ : calcd for $\mathrm{C}_{15} \mathrm{H}_{18} \mathrm{~F}_{2} \mathrm{NaO}_{2}{ }^{+}\left([\mathrm{M}+\mathrm{Na}]^{+}\right)$, 291.1167; found, 291.1161.<smiles>CCOC(=O)CCC/C=C/c1cc(C)cc(C)c1</smiles>

\section{ethyl (E)-6-(3,5-dimethylphenyl)-2,2-difluorohex-5-enoate (3e)}

Following the General Procedure at a $0.2 \mathrm{mmol}$ scale with alkene to afford the tittle compound $\mathbf{3 e}$ as a colorless oil with $70 \%$ yield $(39.5 \mathrm{mg})$.

${ }^{1} \mathrm{H}$ NMR $\left(500 \mathrm{MHz}, \mathrm{CDCl}_{3}\right) \delta 6.95(\mathrm{~s}, 2 \mathrm{H}), 6.86(\mathrm{~s}, 1 \mathrm{H}), 6.37(\mathrm{~d}, J=16.0 \mathrm{~Hz}, 1 \mathrm{H}), 6.15-6.10(\mathrm{~m}, 1 \mathrm{H})$, 4.30 (q, $J=7.0 \mathrm{~Hz}, 2 \mathrm{H}), 2.42-2.37(\mathrm{~m}, 2 \mathrm{H}), 2.29(\mathrm{~s}, 6 \mathrm{H}), 2.27-2.21(\mathrm{~m}, 2 \mathrm{H}), 1.34(\mathrm{t}, J=7.0 \mathrm{~Hz}, 3 \mathrm{H})$. ${ }^{13} \mathbf{C}$ NMR $\left(151 \mathrm{MHz}, \mathrm{CDCl}_{3}\right) \delta 164.2(\mathrm{t}, J=32.9 \mathrm{~Hz}), 138.0,137.0,131.5,129.0,127.0,123.9,115.9(\mathrm{t}$, $J=250.4 \mathrm{~Hz}), 62.8,34.3(\mathrm{t}, J=23.1 \mathrm{~Hz}), 25.1(\mathrm{t}, J=4.5 \mathrm{~Hz}), 21.2,13.9$.

${ }^{19} \mathbf{F}$ NMR $\left(471 \mathrm{MHz}, \mathrm{CDCl}_{3}\right) \delta-106.0(\mathrm{t}, J=17.0 \mathrm{~Hz})$.

HRMS (ESI-TOF) (m/z): calcd for $\mathrm{C}_{16} \mathrm{H}_{20} \mathrm{~F}_{2} \mathrm{NaO}_{2}{ }^{+}\left([\mathrm{M}+\mathrm{Na}]^{+}\right)$, 305.1324; found, 305.1357 .<smiles>CCOC(=O)CCC/C=C/c1ccc(CC)cc1</smiles>

\section{ethyl (E)-6-(4-ethylphenyl)-2,2-difluorohex-5-enoate (3f)}

Following the General Procedure at a $0.2 \mathrm{mmol}$ scale with alkene to afford the tittle compound $\mathbf{3 f}$ as a colorless oil with $80 \%$ yield $(45.1 \mathrm{mg})$.

${ }^{1} \mathbf{H}$ NMR $\left(600 \mathrm{MHz}, \mathrm{CDCl}_{3}\right) \delta 7.25(\mathrm{~d}, J=7.2 \mathrm{~Hz}, 2 \mathrm{H}), 7.13(\mathrm{~d}, J=7.8 \mathrm{~Hz}, 2 \mathrm{H}), 6.40(\mathrm{~d}, J=16.2 \mathrm{~Hz}$, $1 \mathrm{H}), 6.13-6.08(\mathrm{~m}, 1 \mathrm{H}), 4.29$ (q, $J=7.2 \mathrm{~Hz}, 2 \mathrm{H}), 2.62(\mathrm{q}, J=7.8 \mathrm{~Hz}, 2 \mathrm{H}), 2.42-2.38(\mathrm{~m}, 2 \mathrm{H}), 2.28-$ $2.20(\mathrm{~m}, 2 \mathrm{H}), 1.33(\mathrm{t}, J=7.2 \mathrm{~Hz}, 3 \mathrm{H}), 1.22(\mathrm{t}, J=7.8 \mathrm{~Hz}, 3 \mathrm{H})$.

${ }^{13} \mathbf{C}$ NMR $\left(151 \mathrm{MHz}, \mathrm{CDCl}_{3}\right) \delta 164.2(\mathrm{t}, J=32.9 \mathrm{~Hz}), 143.5,134.6,131.3,128.0,126.4,126.0,116.0(\mathrm{t}$, $J=250.7 \mathrm{~Hz}), 62.8,34.4(\mathrm{t}, J=23.3 \mathrm{~Hz}), 28.6,25.1(\mathrm{t}, J=4.8 \mathrm{~Hz}), 15.5,13.9$.

${ }^{19} \mathbf{F}$ NMR $\left(471 \mathrm{MHz}, \mathrm{CDCl}_{3}\right) \delta-106.0(\mathrm{t}, J=16.5 \mathrm{~Hz})$.

HRMS (ESI-TOF) $(\mathrm{m} / \mathrm{z})$ : calcd for $\mathrm{C}_{16} \mathrm{H}_{20} \mathrm{~F}_{2} \mathrm{NaO}_{2}{ }^{+}\left([\mathrm{M}+\mathrm{Na}]^{+}\right)$, 305.1324; found, 305.1327 . 
$\mathrm{CF}_{2} \mathrm{CO}_{2} \mathrm{Et}$

\section{ethyl (E)-2,2-difluoro-6-(4-isopropylphenyl)hex-5-enoate (3g)}

Following the General Procedure at a $0.2 \mathrm{mmol}$ scale with alkene to afford the tittle compound $\mathbf{3 g}$ as a colorless oil with $82 \%$ yield $(48.6 \mathrm{mg})$.

${ }^{1} \mathbf{H}$ NMR $\left(500 \mathrm{MHz}, \mathrm{CDCl}_{3}\right) \delta 7.26(\mathrm{~d}, J=8.5 \mathrm{~Hz}, 2 \mathrm{H}), 7.16(\mathrm{~d}, J=8.0 \mathrm{~Hz}, 2 \mathrm{H}), 6.41(\mathrm{~d}, J=15.0 \mathrm{~Hz}$, 1H), $6.17-6.07$ (m, 1H), 4.30 (q, J = 7.0 Hz, 2H), $2.91-2.85$ (m, 1H), $2.42-2.38$ (m, 2H), $2.29-2.19$ (m, 2H), $1.33(\mathrm{t}, J=7.0 \mathrm{~Hz}, 3 \mathrm{H}), 1.24(\mathrm{~d}, J=6.5 \mathrm{~Hz}, 6 \mathrm{H})$.

${ }^{13}$ C NMR $\left(151 \mathrm{MHz}, \mathrm{CDCl}_{3}\right) \delta 164.2(\mathrm{t}, J=32.6 \mathrm{~Hz}), 148.1,134.7,131.2,126.6,126.5,126.0,116.0$ (t, $J=250.7 \mathrm{~Hz}), 62.8,34.4(\mathrm{t}, J=23.1 \mathrm{~Hz}), 33.8,25.1$ (t, $J=4.8 \mathrm{~Hz}), 23.9,13.9$.

${ }^{19} \mathbf{F}$ NMR $\left(471 \mathrm{MHz}, \mathrm{CDCl}_{3}\right) \delta-106.0(\mathrm{t}, J=17.0 \mathrm{~Hz})$.

HRMS (ESI-TOF) (m/z): calcd for $\mathrm{C}_{17} \mathrm{H}_{22} \mathrm{~F}_{2} \mathrm{NaO}_{2}{ }^{+}\left([\mathrm{M}+\mathrm{Na}]^{+}\right), 319.1480$; found, 319.1474 .<smiles></smiles>

\section{ethyl (E)-6-(4-(tert-butyl)phenyl)-2,2-difluorohex-5-enoate (3h)}

Following the General Procedure at a $0.2 \mathrm{mmol}$ scale with alkene to afford the tittle compound $\mathbf{3 h}$ as a colorless oil with $81 \%$ yield $(50.3 \mathrm{mg})$.

${ }^{1} \mathbf{H}$ NMR $\left(600 \mathrm{MHz}, \mathrm{CDCl}_{3}\right) \delta 7.32(\mathrm{~d}, J=7.8 \mathrm{~Hz}, 2 \mathrm{H}), 7.27(\mathrm{~d}, J=9.0 \mathrm{~Hz}, 2 \mathrm{H}), 6.41(\mathrm{~d}, J=15.8 \mathrm{~Hz}$, $1 \mathrm{H}), 6.14-6.09(\mathrm{~m}, 1 \mathrm{H}), 4.30(\mathrm{q}, J=6.6 \mathrm{~Hz}, 2 \mathrm{H}), 2.42-2.38(\mathrm{~m}, 2 \mathrm{H}), 2.28-2.20(\mathrm{~m}, 2 \mathrm{H}), 1.33(\mathrm{t}, J=$ $7.2 \mathrm{~Hz}, 3 \mathrm{H}), 1.31(\mathrm{~s}, 9 \mathrm{H})$.

${ }^{13}$ C NMR $\left(126 \mathrm{MHz}, \mathrm{CDCl}_{3}\right) \delta 164.2(\mathrm{t}, J=32.6 \mathrm{~Hz}), 150.4,134.3,131.1,126.6,125.7,125.5,116.0(\mathrm{t}$, $J=251.0 \mathrm{~Hz}), 62.9,34.5,34.4(\mathrm{t}, J=22.8 \mathrm{~Hz}), 31.3,25.1(\mathrm{t}, J=4.8 \mathrm{~Hz}), 13.9$.

${ }^{19}$ F NMR $\left(471 \mathrm{MHz}, \mathrm{CDCl}_{3}\right) \delta-106.0(\mathrm{t}, J=16.0 \mathrm{~Hz})$.

HRMS (ESI-TOF) (m/z): calcd for $\mathrm{C}_{18} \mathrm{H}_{24} \mathrm{~F}_{2} \mathrm{NaO}_{2}{ }^{+}\left([\mathrm{M}+\mathrm{Na}]^{+}\right), 333.1367$; found, 333.1367 .<smiles>CCOC(=O)C(F)(F)F</smiles>

ethyl (E)-6-(3,5-di-tert-butylphenyl)-2,2-difluorohex-5-enoate (3i)

Following the General Procedure at a $0.2 \mathrm{mmol}$ scale with alkene to afford the tittle compound $\mathbf{3 i}$ as a colorless oil with $72 \%$ yield $(52.8 \mathrm{mg})$.

${ }^{1} \mathrm{H}$ NMR $\left(500 \mathrm{MHz}, \mathrm{CDCl}_{3}\right) \delta 7.30(\mathrm{~s}, 1 \mathrm{H}), 7.18(\mathrm{~s}, 2 \mathrm{H}), 6.46(\mathrm{~d}, J=15.5 \mathrm{~Hz}, 1 \mathrm{H}), 6.17-6.11(\mathrm{~m}, 1 \mathrm{H})$, 4.30 (q, $J=6.5 \mathrm{~Hz}, 2 \mathrm{H}), 2.44-2.39$ (m, 2H), $2.31-2.21(\mathrm{~m}, 2 \mathrm{H}), 1.34$ (t, $J=7.0 \mathrm{~Hz}, 3 \mathrm{H}), 1.32(\mathrm{~s}, 18 \mathrm{H})$. ${ }^{13} \mathrm{C}$ NMR $\left(151 \mathrm{MHz}, \mathrm{CDCl}_{3}\right) \delta 164.2(\mathrm{t}, J=32.6 \mathrm{~Hz}), 150.9,136.3,132.3,126.6,121.7,120.3,116.0(\mathrm{t}$, $J=250.4 \mathrm{~Hz}), 62.8,34.8,34.4(\mathrm{t}, J=23.3 \mathrm{~Hz}), 31.4,25.1(\mathrm{t}, J=4.7 \mathrm{~Hz}), 13.9$.

${ }^{19} \mathbf{F}$ NMR $\left(471 \mathrm{MHz}, \mathrm{CDCl}_{3}\right) \delta-106.0(\mathrm{t}, J=16.0 \mathrm{~Hz})$.

HRMS (ESI-TOF) (m/z): calcd for $\mathrm{C}_{22} \mathrm{H}_{32} \mathrm{~F}_{2} \mathrm{NaO}_{2}{ }^{+}\left([\mathrm{M}+\mathrm{Na}]^{+}\right)$, 389.2263; found, 389.2267. 
$\mathrm{CF}_{2} \mathrm{CO}_{2} \mathrm{Et}$

\section{ethyl (E)-6-([1,1'-biphenyl]-4-yl)-2,2-difluorohex-5-enoate (3j)}

Following the General Procedure at a $0.2 \mathrm{mmol}$ scale with alkene to afford the tittle compound $\mathbf{3 j}$ as a white solid with $69 \%$ yield $(45.6 \mathrm{mg})$. Mp: $80-81{ }^{\circ} \mathrm{C}$

${ }^{1} \mathbf{H}$ NMR $\left(600 \mathrm{MHz}, \mathrm{CDCl}_{3}\right) \delta 7.59(\mathrm{~d}, J=7.8 \mathrm{~Hz}, 2 \mathrm{H}), 7.54(\mathrm{~d}, J=7.8 \mathrm{~Hz}, 2 \mathrm{H}), 7.44-7.39(\mathrm{~m}, 4 \mathrm{H})$, $7.33(\mathrm{t}, J=7.8 \mathrm{~Hz}, 1 \mathrm{H}), 6.47$ (d, $J=15.6 \mathrm{~Hz}, 1 \mathrm{H}), 6.23-6.18(\mathrm{~m}, 1 \mathrm{H}), 4.30$ (q, $J=7.2 \mathrm{~Hz}, 2 \mathrm{H}), 2.46-$ $2.42(\mathrm{~m}, 2 \mathrm{H}), 2.31-2.23(\mathrm{~m}, 2 \mathrm{H}), 1.34(\mathrm{t}, J=6.6 \mathrm{~Hz}, 3 \mathrm{H})$.

${ }^{13}$ C NMR (151 MHz, $\left.\mathrm{CDCl}_{3}\right) \delta 164.2(\mathrm{t}, J=32.6 \mathrm{~Hz}), 140.7,140.1,136.2,131.0,128.8,127.6,127.3$, 127.2, 126.9, 126.5, 115.9 (t, $J=250.8 \mathrm{~Hz}), 62.9,34.3(\mathrm{t}, J=23.3 \mathrm{~Hz}), 25.2(\mathrm{t}, J=4.8 \mathrm{~Hz}), 13.9$.

${ }^{19}$ F NMR $\left(471 \mathrm{MHz}, \mathrm{CDCl}_{3}\right) \delta-106.0(\mathrm{t}, J=17.0 \mathrm{~Hz})$.

HRMS (ESI-TOF) (m/z): calcd for $\mathrm{C}_{20} \mathrm{H}_{20} \mathrm{~F}_{2} \mathrm{NaO}_{2}{ }^{+}\left([\mathrm{M}+\mathrm{Na}]^{+}\right)$, 353.1324; found, 353.1315 .<smiles>CCOC(=O)CCCC=Cc1ccc(OC)cc1</smiles>

\section{ethyl (E)-2,2-difluoro-6-(4-methoxyphenyl)hex-5-enoate (3k)}

Following the General Procedure at a $0.2 \mathrm{mmol}$ scale with alkene to afford the tittle compound $\mathbf{3 k}$ as a colorless oil with $67 \%$ yield $(38.1 \mathrm{mg})$.

${ }^{1} \mathbf{H}$ NMR $\left(500 \mathrm{MHz}, \mathrm{CDCl}_{3}\right) \delta 7.6(\mathrm{~d}, J=9.0 \mathrm{~Hz}, 2 \mathrm{H}), 6.84(\mathrm{~d}, J=8.5 \mathrm{~Hz}, 2 \mathrm{H}), 6.37(\mathrm{~d}, J=16.0 \mathrm{~Hz}$, 1H), $6.03-5.97$ (m, 1H), 4.29 (q, J = 7.5 Hz, 2H), 3.80 (s, 3H), $2.41-2.37$ (m, 2H), $2.29-2.19$ (m, $2 \mathrm{H}), 1.33(\mathrm{t}, J=7.0 \mathrm{~Hz}, 3 \mathrm{H})$.

${ }^{13}$ C NMR $\left(126 \mathrm{MHz}, \mathrm{CDCl}_{3}\right) \delta 164.2(\mathrm{t}, J=33.1 \mathrm{~Hz}), 159.0,130.7,129.9,127.1,125.2,116.0(\mathrm{t}, J=$ $251.1 \mathrm{~Hz}), 113.9,62.8,55.3,34.4(\mathrm{t}, J=23.6 \mathrm{~Hz}), 25.1(\mathrm{t}, J=5.2 \mathrm{~Hz}), 13.9$.

${ }^{19}$ F NMR $\left(471 \mathrm{MHz}, \mathrm{CDCl}_{3}\right) \delta-106.0(\mathrm{t}, J=16.5 \mathrm{~Hz})$.

HRMS (ESI-TOF) (m/z): calcd for $\mathrm{C}_{15} \mathrm{H}_{18} \mathrm{~F}_{2} \mathrm{NaO}_{3}{ }^{+}\left([\mathrm{M}+\mathrm{Na}]^{+}\right)$, 307.1116; found, 307.1118.<smiles>CCOC(=O)CCCC=Cc1cccc(OC)c1</smiles>

ethyl (E)-2,2-difluoro-6-(3-methoxyphenyl)hex-5-enoate (3I)

Following the General Procedure at a $0.2 \mathrm{mmol}$ scale with alkene to afford the tittle compound $\mathbf{3 I}$ as a colorless oil with $65 \%$ yield $(36.9 \mathrm{mg})$.

${ }^{1} \mathbf{H}$ NMR $\left(500 \mathrm{MHz}, \mathrm{CDCl}_{3}\right) \delta 7.22(\mathrm{t}, J=7.5 \mathrm{~Hz}, 1 \mathrm{H}), 6.93(\mathrm{~d}, J=8.0 \mathrm{~Hz}, 1 \mathrm{H}), 6.87(\mathrm{~s}, 1 \mathrm{H}), 6.79-$ $6.77(\mathrm{~m}, 1 \mathrm{H}), 6.41(\mathrm{~d}, J=16.0 \mathrm{~Hz}, 1 \mathrm{H}), 6.19-6.13(\mathrm{~m}, 1 \mathrm{H}), 4.30(\mathrm{q}, J=7.0 \mathrm{~Hz}, 2 \mathrm{H}), 3.81(\mathrm{~s}, 3 \mathrm{H}), 2.44$ $-2.39(\mathrm{~m}, 2 \mathrm{H}), 2.30-2.21(\mathrm{~m}, 2 \mathrm{H}), 1.34(\mathrm{t}, J=7.0 \mathrm{~Hz}, 3 \mathrm{H})$.

${ }^{13} \mathbf{C}$ NMR (126 MHz, $\left.\mathrm{CDCl}_{3}\right) \delta 164.2(\mathrm{t}, J=32.5 \mathrm{~Hz}), 159.8,138.6,131.3,129.5,127.8,118.7,115.9(\mathrm{t}$, $J=251.1 \mathrm{~Hz}), 112.9,111.4,62.9,55.2,34.2(\mathrm{t}, J=23.1 \mathrm{~Hz}), 25.1(\mathrm{t}, J=4.8 \mathrm{~Hz}), 13.9$.

${ }^{19}$ F NMR $\left(471 \mathrm{MHz}, \mathrm{CDCl}_{3}\right) \delta-106.0(\mathrm{t}, J=16.5 \mathrm{~Hz})$.

HRMS (ESI-TOF) $(\mathrm{m} / \mathrm{z})$ : calcd for $\mathrm{C}_{15} \mathrm{H}_{18} \mathrm{~F}_{2} \mathrm{NaO}_{3}{ }^{+}\left([\mathrm{M}+\mathrm{Na}]^{+}\right)$, 317.1116; found, 307.1165. 
$\mathrm{MeS} \mathrm{CF}_{2} \mathrm{CO}_{2} \mathrm{Et}$

ethyl (E)-2,2-difluoro-6-(4-(methylthio)phenyl)hex-5-enoate (3m)

Following the General Procedure at a $0.2 \mathrm{mmol}$ scale with alkene to afford the tittle compound $\mathbf{3 m}$ as a colorless oil with $71 \%$ yield $(42.6 \mathrm{mg})$.

${ }^{1} \mathbf{H}$ NMR $\left(500 \mathrm{MHz}, \mathrm{CDCl}_{3}\right) \delta 7.25(\mathrm{~d}, J=8.5 \mathrm{~Hz}, 2 \mathrm{H}), 7.19(\mathrm{~d}, J=8.5 \mathrm{~Hz}, 2 \mathrm{H}), 6.38(\mathrm{~d}, J=16.0 \mathrm{~Hz}$, $1 \mathrm{H}), 6.14-6.08$ (m, 1H), 4.29 (q, J = 7.0 Hz, 2H), 2.48 (s, 3H), $2.43-2.38(\mathrm{~m}, 2 \mathrm{H}), 2.30-2.20$ (m, $2 \mathrm{H}), 1.33(\mathrm{t}, J=7.0 \mathrm{~Hz}, 3 \mathrm{H})$.

${ }^{13}$ C NMR $\left(151 \mathrm{MHz}, \mathrm{CDCl}_{3}\right) \delta 164.2(\mathrm{t}, J=32.8 \mathrm{~Hz}), 137.4,134.1,130.8,126.9,126.8,126.4,115.9(\mathrm{t}$, $J=251.9 \mathrm{~Hz}), 62.9,34.3(\mathrm{t}, J=23.4 \mathrm{~Hz}), 25.1(\mathrm{t}, J=4.8 \mathrm{~Hz}), 15.9,13.9$.

${ }^{19}$ F NMR $\left(471 \mathrm{MHz}, \mathrm{CDCl}_{3}\right) \delta-106.0(\mathrm{t}, J=16.0 \mathrm{~Hz})$.

HRMS (ESI-TOF) (m/z): calcd for $\mathrm{C}_{15} \mathrm{H}_{18} \mathrm{~F}_{2} \mathrm{NaO}_{2} \mathrm{~S}^{+}\left([\mathrm{M}+\mathrm{Na}]^{+}\right)$, 323.0888; found, 323.0880 .

$\mathrm{Me}_{2} \mathrm{~N} \mathrm{CF}_{2} \mathrm{CO}_{2} \mathrm{Et}$

ethyl (E)-6-(4-(dimethylamino)phenyl)-2,2-difluorohex-5-enoate (3n)

Following the General Procedure at a $0.2 \mathrm{mmol}$ scale with alkene to afford the tittle compound $\mathbf{3 n}$ as a colorless oil with $40 \%$ yield $(23.8 \mathrm{mg})$.

${ }^{1} \mathbf{H}$ NMR $\left(500 \mathrm{MHz}, \mathrm{CDCl}_{3}\right) \delta 7.22(\mathrm{~d}, J=8.5 \mathrm{~Hz}, 2 \mathrm{H}), 6.67(\mathrm{~d}, J=8.5 \mathrm{~Hz}, 2 \mathrm{H}), 6.34(\mathrm{~d}, J=16.0 \mathrm{~Hz}$, $1 \mathrm{H}), 5.96-5.90(\mathrm{~m}, 1 \mathrm{H}), 2.94(\mathrm{~s}, 6 \mathrm{H}), 4.28(\mathrm{q}, J=7.0 \mathrm{~Hz}, 2 \mathrm{H}), 2.39-2.35(\mathrm{~m}, 2 \mathrm{H}), 2.28-2.18(\mathrm{~m}$, $2 \mathrm{H}), 1.33(\mathrm{t}, J=7.0 \mathrm{~Hz}, 3 \mathrm{H})$.

${ }^{13} \mathbf{C}$ NMR $\left(151 \mathrm{MHz}, \mathrm{CDCl}_{3}\right) \delta 164.3(\mathrm{t}, J=32.9 \mathrm{~Hz}), 149.9,131.2,126.9,125.7,123.0,116.0(\mathrm{t}, J=$ $250.0 \mathrm{~Hz}), 112.5,62.8,40.5,34.6(\mathrm{t}, J=22.9 \mathrm{~Hz}), 25.1(\mathrm{t}, J=5.0 \mathrm{~Hz}), 13.9$.

${ }^{19} \mathbf{F}$ NMR $\left(471 \mathrm{MHz}, \mathrm{CDCl}_{3}\right) \delta-106.0(\mathrm{t}, J=17.4 \mathrm{~Hz})$.

HRMS (ESI-TOF) (m/z): calcd for $\mathrm{C}_{16} \mathrm{H}_{22} \mathrm{~F}_{2} \mathrm{NO}_{2}{ }^{+}\left([\mathrm{M}+\mathrm{H}]^{+}\right)$, 298.1613; found, 298.1604.<smiles>CCOC(=O)CCC/C=C/c1ccc(F)cc1</smiles>

ethyl (E)-2,2-difluoro-6-(4-fluorophenyl)hex-5-enoate (3o)

Following the General Procedure at a $0.2 \mathrm{mmol}$ scale with alkene to afford the tittle compound $\mathbf{3 o}$ as a colorless oil with $68 \%$ yield $(37.0 \mathrm{mg})$.

${ }^{1} \mathbf{H}$ NMR $\left(500 \mathrm{MHz}, \mathrm{CDCl}_{3}\right) \delta 7.30-7.28(\mathrm{~m}, 2 \mathrm{H}), 7.00-6.97(\mathrm{~m}, 2 \mathrm{H}), 6.39(\mathrm{~d}, J=15.5 \mathrm{~Hz}, 1 \mathrm{H}), 6.10$ $-6.04(\mathrm{~m}, 1 \mathrm{H}), 4.29$ (q, J = 7.0 Hz, 2H), $2.43-2.38(\mathrm{~m}, 2 \mathrm{H}), 2.30-2.20(\mathrm{~m}, 2 \mathrm{H}), 1.33(\mathrm{t}, J=7.5 \mathrm{~Hz}$, $3 \mathrm{H})$.

${ }^{13}$ C NMR (151 MHz, $\left.\mathrm{CDCl}_{3}\right) \delta 164.2(\mathrm{t}, J=32.8 \mathrm{~Hz}), 162.1(\mathrm{~d}, J=246.4 \mathrm{~Hz}), 133.3(\mathrm{~d}, J=3.3 \mathrm{~Hz})$, 130.2, 127.5 (d, $J=8.0 \mathrm{~Hz}), 127.2$ (d, $J=2.4 \mathrm{~Hz}), 115.9$ (d, $J=250.7 \mathrm{~Hz}), 115.4$ (d, $J=21.6 \mathrm{~Hz}), 62.9$, $34.2(\mathrm{t}, J=23.1 \mathrm{~Hz}), 25.1(\mathrm{t}, J=4.8 \mathrm{~Hz}), 13.9$.

${ }^{19} \mathbf{F}$ NMR (471 MHz, $\left.\mathrm{CDCl}_{3}\right) \delta-106.0(\mathrm{t}, J=17.4 \mathrm{~Hz}),(-114.9)-(-115.0)(\mathrm{m})$.

HRMS (ESI-TOF) (m/z): calcd for $\mathrm{C}_{14} \mathrm{H}_{15} \mathrm{~F}_{3} \mathrm{NaO}_{2}{ }^{+}\left([\mathrm{M}+\mathrm{Na}]^{+}\right), 295.0916$; found, 295.0923 . 
<smiles>CCOC(=O)C(F)(F)F</smiles>

ethyl (E)-2,2-difluoro-6-(2-fluorophenyl)hex-5-enoate (3p)

Following the General Procedure at a $0.2 \mathrm{mmol}$ scale with alkene to afford the tittle compound $\mathbf{3 p}$ as a colorless oil with $60 \%$ yield $(32.6 \mathrm{mg})$.

${ }^{1} \mathbf{H}$ NMR $\left(600 \mathrm{MHz}, \mathrm{CDCl}_{3}\right) \delta 7.40(\mathrm{td}, J=7.8,1.8 \mathrm{~Hz}, 1 \mathrm{H}), 7.20-7.16(\mathrm{~m}, 1 \mathrm{H}), 7.09-7.06(\mathrm{~m}, 1 \mathrm{H})$, $7.03-7.00(\mathrm{~m}, 1 \mathrm{H}), 6.58(\mathrm{~d}, J=15.6 \mathrm{~Hz}, 1 \mathrm{H}), 6.27-6.23(\mathrm{~m}, 1 \mathrm{H}), 4.31(\mathrm{q}, J=7.2 \mathrm{~Hz}, 2 \mathrm{H}), 2.47-2.43$ (m, 2H), $2.31-2.23(\mathrm{~m}, 2 \mathrm{H}), 1.34$ (t, $J=7.2 \mathrm{~Hz}, 3 \mathrm{H})$.

${ }^{13} \mathrm{C}$ NMR $\left(151 \mathrm{MHz}, \mathrm{CDCl}_{3}\right) \delta 164.2(\mathrm{t}, J=32.8 \mathrm{~Hz}), 160.0(\mathrm{t}, J=249.0 \mathrm{~Hz}), 130.2(\mathrm{t}, J=4.7 \mathrm{~Hz})$, $128.5(\mathrm{t}, J=8.6 \mathrm{~Hz}), 127.2(\mathrm{t}, J=3.6 \mathrm{~Hz}), 124.8(\mathrm{t}, J=12.2 \mathrm{~Hz}), 124.1$ (t, $J=3.6 \mathrm{~Hz}), 123.9$ (t, $J=3.3$ Hz), 115.9 (t, $J=250.4 \mathrm{~Hz}), 115.7(\mathrm{~d}, J=22.0 \mathrm{~Hz}), 62.9,34.2(\mathrm{t}, J=23.4 \mathrm{~Hz}), 25.5(\mathrm{t}, J=4.8 \mathrm{~Hz}), 13.9$. ${ }^{19}$ F NMR $\left(471 \mathrm{MHz}, \mathrm{CDCl}_{3}\right) \delta-106.0(\mathrm{t}, J=16.5 \mathrm{~Hz}) .(-118.5)-(-118.6)(\mathrm{m})$.

HRMS (ESI-TOF) (m/z): calcd for $\mathrm{C}_{14} \mathrm{H}_{15} \mathrm{~F}_{3} \mathrm{NaO}_{2}{ }^{+}\left([\mathrm{M}+\mathrm{Na}]^{+}\right), 295.0916$; found, 295.0912 .<smiles>CCOC(=O)CCC/C=C/c1ccc(Cl)cc1</smiles>

\section{ethyl (E)-6-(4-chlorophenyl)-2,2-difluorohex-5-enoate (3q)}

Following the General Procedure at a $0.2 \mathrm{mmol}$ scale with alkene to afford the tittle compound $\mathbf{3 q}$ as a colorless oil with $76 \%$ yield $(43.9 \mathrm{mg})$.

${ }^{1} \mathbf{H}$ NMR $\left(600 \mathrm{MHz}, \mathrm{CDCl}_{3}\right) \delta 7.27-7.24(\mathrm{~m}, 4 \mathrm{H}), 6.38(\mathrm{~d}, J=15.6 \mathrm{~Hz}, 1 \mathrm{H}), 6.16-6.11(\mathrm{~m}, 1 \mathrm{H}), 4.29$ (q, $J=7.2 \mathrm{~Hz}, 2 \mathrm{H}), 2.43-2.39(\mathrm{~m}, 2 \mathrm{H}), 2.29-2.21(\mathrm{~m}, 2 \mathrm{H}), 1.33(\mathrm{t}, J=7.2 \mathrm{~Hz}, 3 \mathrm{H})$.

${ }^{13} \mathbf{C}$ NMR $\left(151 \mathrm{MHz}, \mathrm{CDCl}_{3}\right) \delta 164.1(\mathrm{t}, J=32.8 \mathrm{~Hz}), 135.6,132.9,130.2,128.7,128.2,127.2,115.9(\mathrm{t}$, $J=250.5 \mathrm{~Hz}), 62.9,34.1(\mathrm{t}, J=23.3 \mathrm{~Hz}), 25.1(\mathrm{t}, J=4.8 \mathrm{~Hz}), 13.9$.

${ }^{19}$ F NMR $\left(471 \mathrm{MHz}, \mathrm{CDCl}_{3}\right) \delta-106.0(\mathrm{t}, J=17.4 \mathrm{~Hz})$.

HRMS (ESI-TOF) $(\mathrm{m} / \mathrm{z})$ : calcd for $\mathrm{C}_{14} \mathrm{H}_{15} \mathrm{ClFNaO}_{2}{ }^{+}\left([\mathrm{M}+\mathrm{Na}]^{+}\right), 311.0621$; found, 311.0621 .<smiles>CCOC(=O)CCC/C=C/c1ccc(Cl)c(Cl)c1</smiles>

\section{ethyl (E)-6-(3,4-dichlorophenyl)-2,2-difluorohex-5-enoate (3r)}

Following the General Procedure at a $0.2 \mathrm{mmol}$ scale with alkene to afford the tittle compound $\mathbf{3 r}$ as a colorless oil with $78 \%$ yield $(50.4 \mathrm{mg})$.

${ }^{1}$ H NMR $\left(500 \mathrm{MHz}, \mathrm{CDCl}_{3}\right) \delta 7.41(\mathrm{~s}, 1 \mathrm{H}), 7.36(\mathrm{~d}, J=8.5 \mathrm{~Hz}, 1 \mathrm{H}), 7.15(\mathrm{~d}, J=8.5 \mathrm{~Hz}, 1 \mathrm{H}), 6.34(\mathrm{~d}$, $J=16.0 \mathrm{~Hz}, 1 \mathrm{H}), 6.20-6.14(\mathrm{~m}, 1 \mathrm{H}), 4.30(\mathrm{q}, J=7.0 \mathrm{~Hz}, 2 \mathrm{H}), 2.45-2.40$ (m, 2H), $2.30-2.20(\mathrm{~m}, 2 \mathrm{H})$, $1.34(\mathrm{t}, J=7.0 \mathrm{~Hz}, 3 \mathrm{H})$.

${ }^{13} \mathbf{C}$ NMR $\left(151 \mathrm{MHz}, \mathrm{CDCl}_{3}\right) \delta 164.1(\mathrm{t}, J=32.5 \mathrm{~Hz}), 137.2,132.7,130.9,130.4,129.7,129.2,127.8$, 125.3, $115.8(\mathrm{t}, J=250.7 \mathrm{~Hz}), 62.9,34.0(\mathrm{t}, J=23.3 \mathrm{~Hz}), 25.1$ (t, $J=4.7 \mathrm{~Hz}), 13.9$.

${ }^{19} \mathbf{F}$ NMR $\left(471 \mathrm{MHz}, \mathrm{CDCl}_{3}\right) \delta-106.0(\mathrm{t}, J=16.0 \mathrm{~Hz})$.

HRMS (ESI-TOF) (m/z): calcd for $\mathrm{C}_{14} \mathrm{H}_{14} \mathrm{Cl}_{2} \mathrm{~F}_{2} \mathrm{NaO}_{2}{ }^{+}\left([\mathrm{M}+\mathrm{Na}]^{+}\right), 345.0231$; found, 345.0221 . 
作

\section{ethyl (E)-2,2-difluoro-6-(4-(trifluoromethoxy)phenyl)hex-5-enoate (3s)}

Following the General Procedure at a $0.2 \mathrm{mmol}$ scale with alkene to afford the tittle compound $3 \mathrm{~s}$ as a colorless oil with $70 \%$ yield $(47.3 \mathrm{mg})$.

${ }^{1} \mathbf{H}$ NMR $\left(500 \mathrm{MHz}, \mathrm{CDCl}_{3}\right) \delta 7.34(\mathrm{~d}, J=8.5 \mathrm{~Hz}, 2 \mathrm{H}), 7.15(\mathrm{~d}, J=8.5 \mathrm{~Hz}, 2 \mathrm{H}), 6.42(\mathrm{~d}, J=16.0 \mathrm{~Hz}$, $1 \mathrm{H}), 6.17-6.11(\mathrm{~m}, 1 \mathrm{H}), 4.30$ (q, $J=7.5 \mathrm{~Hz}, 2 \mathrm{H}), 2.45-2.40(\mathrm{~m}, 2 \mathrm{H}), 2.31-2.21(\mathrm{~m}, 2 \mathrm{H}), 1.34(\mathrm{t}, J=$ $7.5 \mathrm{~Hz}, 3 \mathrm{H})$.

${ }^{13} \mathbf{C}$ NMR $\left(126 \mathrm{MHz}, \mathrm{CDCl}_{3}\right) \delta 164.2(\mathrm{t}, J=33.3 \mathrm{~Hz}), 148.3,135.9,133.0,128.6,127.2,121.1,120.5$ (q, $J=275.1 \mathrm{~Hz}), 115.9$ (t, $J=250.6 \mathrm{~Hz}), 62.9,34.1$ (t, $J=23.4 \mathrm{~Hz}), 25.1$ (t, $J=4.8 \mathrm{~Hz}), 13.9$.

${ }^{19} \mathbf{F}$ NMR $\left(471 \mathrm{MHz}, \mathrm{CDCl}_{3}\right) \delta-57.9(\mathrm{~s}),(-106.0)(\mathrm{t}, J=16.5 \mathrm{~Hz})$.

HRMS (ESI-TOF) $(\mathrm{m} / \mathrm{z})$ : calcd for $\mathrm{C}_{15} \mathrm{H}_{16} \mathrm{~F}_{5} \mathrm{O}_{3}{ }^{+}\left([\mathrm{M}+\mathrm{H}]^{+}\right), 339.1014$; found, 399.1048 .<smiles>CCOC(=O)CCCC=Cc1ccc(C(F)(F)F)cc1</smiles>

\section{ethyl (E)-2,2-difluoro-6-(4-(trifluoromethyl)phenyl)hex-5-enoate (3t)}

Following the General Procedure at a $0.2 \mathrm{mmol} \mathrm{s}$ cale with alkene to afford the tittle compound $\mathbf{3 t}$ as a colorless oil with $72 \%$ yield $(46.4 \mathrm{mg})$.

${ }^{1} \mathbf{H}$ NMR $\left(600 \mathrm{MHz}, \mathrm{CDCl}_{3}\right) \delta 7.55(\mathrm{~d}, J=7.8 \mathrm{~Hz}, 2 \mathrm{H}), 7.42(\mathrm{~d}, J=7.8 \mathrm{~Hz}, 2 \mathrm{H}), 6.47(\mathrm{~d}, J=16.2 \mathrm{~Hz}$, $1 \mathrm{H}), 6.30-6.25(\mathrm{~m}, 1 \mathrm{H}), 4.30$ (q, $J=6.6 \mathrm{~Hz}, 2 \mathrm{H}), 2.48-2.44(\mathrm{~m}, 2 \mathrm{H}), 2.31-2.23(\mathrm{~m}, 2 \mathrm{H}), 1.34(\mathrm{t}, J=$ $7.2 \mathrm{~Hz}, 3 \mathrm{H})$.

${ }^{13} \mathbf{C ~ N M R}\left(126 \mathrm{MHz}, \mathrm{CDCl}_{3}\right) \delta 164.1(\mathrm{t}, J=32.6 \mathrm{~Hz}), 140.5,130.3,130.2,129.2(\mathrm{q}, J=32.6 \mathrm{~Hz}), 126.2$, 125.5 (q, $J=3.8 \mathrm{~Hz}), 124.2$ ( q, $J=272.2 \mathrm{~Hz}), 115.8(\mathrm{t}, J=251.2 \mathrm{~Hz}), 62.9,34.0(\mathrm{t}, J=23.2 \mathrm{~Hz}), 25.1$ (t, $J=5.3 \mathrm{~Hz}), 13.9$.

${ }^{19}$ F NMR $\left(471 \mathrm{MHz}, \mathrm{CDCl}_{3}\right) \delta-62.5(\mathrm{~s}),-106.0(\mathrm{t}, J=16.5 \mathrm{~Hz})$.

HRMS (ESI-TOF) (m/z): calcd for $\mathrm{C}_{15} \mathrm{H}_{15} \mathrm{~F}_{5} \mathrm{NaO}_{2}{ }^{+}\left([\mathrm{M}+\mathrm{Na}]^{+}\right)$, 345.0884; found, 345.0871 .<smiles></smiles>

\section{ethyl (E)-2,2-difluoro-6-(3-(trifluoromethyl)phenyl)hex-5-enoate (3u)}

Following the General Procedure at a $0.2 \mathrm{mmol}$ scale with alkene to afford the tittle compound $\mathbf{3 u}$ as a colorless oil with $78 \%$ yield $(50.2 \mathrm{mg})$.

${ }^{1} \mathbf{H}$ NMR $\left(500 \mathrm{MHz}, \mathrm{CDCl}_{3}\right) \delta 7.57(\mathrm{~s}, 1 \mathrm{H}), 7.50-7.46(\mathrm{~m}, 2 \mathrm{H}), 7.43-7.39(\mathrm{~m}, 1 \mathrm{H}), 6.47$ (d, $J=15.5$ $\mathrm{Hz}, 1 \mathrm{H}), 6.28-6.22(\mathrm{~m}, 1 \mathrm{H}), 4.30(\mathrm{q}, J=7.5 \mathrm{~Hz}, 2 \mathrm{H}), 2.47-2.43(\mathrm{~m}, 2 \mathrm{H}), 2.32-2.22(\mathrm{~m}, 2 \mathrm{H}), 1.34(\mathrm{t}$, $J=7.0 \mathrm{~Hz}, 3 \mathrm{H})$.

${ }^{13}$ C NMR $\left(151 \mathrm{MHz}, \mathrm{CDCl}_{3}\right) \delta 164.1(\mathrm{t}, J=32.8 \mathrm{~Hz}), 137.9,131.0(\mathrm{q}, J=32.3 \mathrm{~Hz}), 130.1,129.6,129.2$, 129.0, 124.1 (q, $J=273.8 \mathrm{~Hz}), 123.8$ (q, $J=3.9 \mathrm{~Hz}), 122.7$ (q, $J=3.8 \mathrm{~Hz}), 115.8(\mathrm{t}, J=250.7 \mathrm{~Hz}), 62.9$, $34.0(\mathrm{t}, J=23.3 \mathrm{~Hz}), 25.1(\mathrm{t}, J=4.8 \mathrm{~Hz}), 13.9$.

${ }^{19}$ F NMR $\left(471 \mathrm{MHz}, \mathrm{CDCl}_{3}\right) \delta-62.8(\mathrm{~s}),-106.0(\mathrm{t}, J=17.0 \mathrm{~Hz})$.

HRMS (ESI-TOF) (m/z): calcd for $\mathrm{C}_{15} \mathrm{H}_{15} \mathrm{~F}_{5} \mathrm{NaO}_{2}{ }^{+}\left([\mathrm{M}+\mathrm{Na}]^{+}\right)$, 345.0884; found, 345.0856 . 


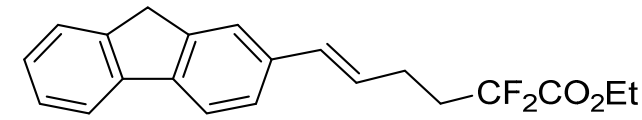

\section{ethyl (E)-6-(9H-fluoren-2-yl)-2,2-difluorohex-5-enoate (3v)}

Following the General Procedure at a $0.2 \mathrm{mmol}$ scale with alkene to afford the tittle compound $\mathbf{3 v}$ as a white solid with $52 \%$ yield $(35.6 \mathrm{mg})$. Mp: $82-83{ }^{\circ} \mathrm{C}$

${ }^{1} \mathbf{H}$ NMR $\left(500 \mathrm{MHz}, \mathrm{CDCl}_{3}\right) \delta 7.75(\mathrm{~d}, J=7.5 \mathrm{~Hz}, 1 \mathrm{H}), 7.70(\mathrm{~d}, J=8.0 \mathrm{~Hz}, 1 \mathrm{H}), 7.53-7.52(\mathrm{~m}, 2 \mathrm{H})$, $7.38-7.33(\mathrm{~m}, 2 \mathrm{H}), 7.29(\mathrm{t}, J=7.5,1 \mathrm{H}), 6.50(\mathrm{~d}, J=16.0 \mathrm{~Hz}, 1 \mathrm{H}), 6.23-6.17(\mathrm{~m}, 1 \mathrm{H}), 4.29(\mathrm{q}, J=7.0$ $\mathrm{Hz}, 2 \mathrm{H}), 3.88(\mathrm{~s}, 2 \mathrm{H}), 2.47-2.42(\mathrm{~m}, 2 \mathrm{H}), 2.33-2.23(\mathrm{~m}, 2 \mathrm{H}), 1.33(\mathrm{t}, J=7.0 \mathrm{~Hz}, 3 \mathrm{H})$.

${ }^{13}$ C NMR $\left(126 \mathrm{MHz}, \mathrm{CDCl}_{3}\right) \delta 164.2(\mathrm{t}, J=32.6 \mathrm{~Hz}), 143.6,143.4,141.4,141.0,135.8,131.7,126.8$, 126.8, 126.6, 125.1, 125.0, 122.4, 119.9, 116.0 (t, $J=251.1 \mathrm{~Hz}), 62.9,36.8,34.3(\mathrm{t}, J=23.1 \mathrm{~Hz}), 25.2$ $(\mathrm{t}, J=4.9 \mathrm{~Hz}), 13.9$.

${ }^{19}$ F NMR $\left(471 \mathrm{MHz}, \mathrm{CDCl}_{3}\right) \delta-105.9(\mathrm{t}, J=16.0 \mathrm{~Hz})$.

HRMS (ESI-TOF) $(\mathrm{m} / \mathrm{z})$ : calcd for $\mathrm{C}_{21} \mathrm{H}_{20} \mathrm{~F}_{2} \mathrm{NaO}_{2}{ }^{+}\left([\mathrm{M}+\mathrm{Na}]^{+}\right), 365.1324$; found, 365.1325 .<smiles>CCOC(=O)CCC/C=C/c1ccccn1</smiles>

\section{ethyl (E)-2,2-difluoro-6-(pyridin-2-yl)hex-5-enoate (3w)}

Following the General Procedure at a $0.2 \mathrm{mmol}$ scale with alkene to afford the tittle compound $\mathbf{3 w}$ as a colorless oil with $36 \%$ yield $(18.3 \mathrm{mg})$.

${ }^{1} \mathbf{H}$ NMR $\left(500 \mathrm{MHz}, \mathrm{CDCl}_{3}\right) \delta 8.53(\mathrm{~d}, J=4.5 \mathrm{~Hz}, 1 \mathrm{H}), 7.62(\mathrm{td}, J=7.5,2.0 \mathrm{~Hz}, 1 \mathrm{H}), 7.23(\mathrm{~d}, J=8.0$ $\mathrm{Hz}, 1 \mathrm{H}), 7.13-7.11(\mathrm{~m}, 1 \mathrm{H}), 6.73-6.68(\mathrm{~m}, 1 \mathrm{H}), 6.53$ (d, J=15.5 Hz, 1H), 4.30 (q, J = 7.0 Hz, 2H), $2.50-2.45(\mathrm{~m}, 2 \mathrm{H}), 2.34-2.24(\mathrm{~m}, 2 \mathrm{H}), 1.34(\mathrm{t}, J=7.0 \mathrm{~Hz}, 3 \mathrm{H})$.

${ }^{13} \mathbf{C}$ NMR $\left(126 \mathrm{MHz}, \mathrm{CDCl}_{3}\right) \delta 164.1(\mathrm{t}, J=32.5 \mathrm{~Hz}), 155.3,149.5,136.5,132.2,131.2,122.0,121.3$, $115.8(\mathrm{t}, J=251.1 \mathrm{~Hz}), 62.9,33.9(\mathrm{t}, J=23.7 \mathrm{~Hz}), 24.8(\mathrm{t}, J=5.3 \mathrm{~Hz}), 13.9$.

${ }^{19}$ F NMR $\left(471 \mathrm{MHz}, \mathrm{CDCl}_{3}\right) \delta-106.1(\mathrm{t}, J=16.0 \mathrm{~Hz})$.

HRMS (ESI-TOF) (m/z): calcd for $\mathrm{C}_{13} \mathrm{H}_{16} \mathrm{~F}_{2} \mathrm{NO}_{2}{ }^{+}\left([\mathrm{M}+\mathrm{H}]^{+}\right), 256.1144$; found, 256.1149 .<smiles>CCOC(=O)CCCC=Cc1ccc(C=CCCCCC(=O)OCC)cc1</smiles>

\section{diethyl 6,6'-(1,4-phenylene)(5E,5'E)-bis(2,2-difluorohex-5-enoate) (3x)}

Following the General Procedure at a $0.2 \mathrm{mmol}$ scale with alkene to afford the tittle compound $\mathbf{3 x}$ as a colorless oil with $53 \%$ yield $(36.1 \mathrm{mg})$.

${ }^{1} \mathbf{H}$ NMR $\left(600 \mathrm{MHz} \mathrm{CDCl}_{3}\right) \delta 7.26(\mathrm{~d}, J=2.4 \mathrm{~Hz}, 4 \mathrm{H}), 6.40(\mathrm{~d}, J=16.2 \mathrm{~Hz}, 2 \mathrm{H}), 6.17-6.12(\mathrm{~m}, 2 \mathrm{H})$, 4.29 (q, $J=7.2 \mathrm{~Hz}, 4 \mathrm{H}), 2.43-2.39(\mathrm{~m}, 4 \mathrm{H}), 2.29-2.21(\mathrm{~m}, 4 \mathrm{H}), 1.33(\mathrm{t}, J=7.2 \mathrm{~Hz}, 6 \mathrm{H})$.

${ }^{13}$ C NMR $\left(151 \mathrm{MHz} \mathrm{CDCl}_{3}\right) \delta 164.2(\mathrm{t}, J=32.8 \mathrm{~Hz}), 136.2,131.0,127.2,126.2,115.9(\mathrm{t}, J=250.4$ $\mathrm{Hz}), 62.9,34.3$ (t, $J=23.3 \mathrm{~Hz}), 25.1(\mathrm{t}, J=4.8 \mathrm{~Hz}), 13.9$.

${ }^{19}$ F NMR (471 MHz, $\left.\mathrm{CDCl}_{3}\right) \delta-106.0(\mathrm{t}, J=17.4 \mathrm{~Hz})$.

HRMS (ESI-TOF) $(\mathrm{m} / \mathrm{z})$ : calcd for $\mathrm{C}_{22} \mathrm{H}_{26} \mathrm{~F}_{4} \mathrm{NaO}_{4}{ }^{+}\left([\mathrm{M}+\mathrm{Na}]^{+}\right), 453.1659$; found, 453.1653. 
$\sim \mathrm{CF}_{2} \mathrm{CO}_{2} \mathrm{Et}$

\section{ethyl (E)-2,2-difluoro-5-phenylpent-4-enoate (3y)}

Following the General Procedure at a $0.2 \mathrm{mmol}$ scale with alkene to afford the tittle compound $\mathbf{3 y}$ as a colorless oil with $75 \%$ yield $(36.0 \mathrm{mg})$.

${ }^{1} \mathbf{H}$ NMR $\left(600 \mathrm{MHz}, \mathrm{CDCl}_{3}\right) \delta 7.36(\mathrm{~d}, J=7.2 \mathrm{~Hz}, 2 \mathrm{H}), 7.31(\mathrm{t}, J=7.8 \mathrm{~Hz}, 2 \mathrm{H}), 7.26-7.24(\mathrm{~m}, 1 \mathrm{H})$, $6.56(\mathrm{~d}, J=15.6 \mathrm{~Hz}, 1 \mathrm{H}), 6.12-6.07(\mathrm{~m}, 1 \mathrm{H}), 4.32(\mathrm{q}, J=7.2 \mathrm{~Hz}, 2 \mathrm{H}), 3.03-2.96(\mathrm{~m}, 2 \mathrm{H}), 1.32(\mathrm{t}, J$ $=7.2 \mathrm{~Hz}, 3 \mathrm{H})$.

${ }^{13} \mathrm{C}$ NMR $\left(126 \mathrm{MHz}, \mathrm{CDCl}_{3}\right) \delta 163.9(\mathrm{t}, J=32.5 \mathrm{~Hz}), 136.5,128.6,127.9,126.4,118.0(\mathrm{t}, J=5.3 \mathrm{~Hz})$, $115.3(\mathrm{t}, J=252.1 \mathrm{~Hz}), 62.8,38.5(\mathrm{t}, J=24.2 \mathrm{~Hz}) 14.0$.

${ }^{19}$ F NMR $\left(471 \mathrm{MHz}, \mathrm{CDCl}_{3}\right) \delta-105.2(\mathrm{t}, J=16.0 \mathrm{~Hz})$.

HRMS (ESI-TOF) (m/z): calcd for $\mathrm{C}_{13} \mathrm{H}_{14} \mathrm{~F}_{2} \mathrm{NaO}_{2}{ }^{+}\left([\mathrm{M}+\mathrm{Na}]^{+}\right)$, 263.0854; found, 263.0839.<smiles>CCOC(=O)CC/C=C/c1ccc(OC)cc1</smiles>

\section{ethyl (E)-2,2-difluoro-5-(4-methoxyphenyl)pent-4-enoate (3z)}

Following the General Procedure at a $0.2 \mathrm{mmol}$ scale with alkene to afford the tittle compound $\mathbf{3 z}$ as a colorless oil with $71 \%$ yield $(38.3 \mathrm{mg})$.

${ }^{1} \mathbf{H}$ NMR $\left(500 \mathrm{MHz}, \mathrm{CDCl}_{3}\right) \delta 7.29(\mathrm{~d}, J=8.5 \mathrm{~Hz}, 2 \mathrm{H}), 6.85(\mathrm{~d}, J=8.5 \mathrm{~Hz}, 2 \mathrm{H}), 6.50(\mathrm{~d}, J=16.0 \mathrm{~Hz}$, $1 \mathrm{H}), 5.97-5.91(\mathrm{~m}, 1 \mathrm{H}), 4.31(\mathrm{q}, J=7.0 \mathrm{~Hz}, 2 \mathrm{H}), 3.81(\mathrm{~s}, 3 \mathrm{H}), 3.00-2.92(\mathrm{~m}, 2 \mathrm{H}), 1.32(\mathrm{t}, J=7.0 \mathrm{~Hz}$, $3 \mathrm{H})$.

${ }^{13}$ C NMR $\left(151 \mathrm{MHz}, \mathrm{CDCl}_{3}\right) \delta 163.9(\mathrm{t}, J=32.6 \mathrm{~Hz}), 159.5,135.8,129.3,127.6,115.7(\mathrm{t}, J=5.3 \mathrm{~Hz})$, $115.4(\mathrm{t}, J=251.4 \mathrm{~Hz}), 114.0,62.8,55.3,38.5(\mathrm{t}, J=23.9 \mathrm{~Hz}), 14.0$.

${ }^{19} \mathbf{F}$ NMR $\left(471 \mathrm{MHz}, \mathrm{CDCl}_{3}\right) \delta-105.3(\mathrm{t}, J=16.0 \mathrm{~Hz})$.

HRMS (ESI-TOF) (m/z): calcd for $\mathrm{C}_{14} \mathrm{H}_{16} \mathrm{~F}_{2} \mathrm{NaO}_{3}{ }^{+}\left([\mathrm{M}+\mathrm{Na}]^{+}\right), 293.0960$; found, 293.0955 .<smiles>CCOC(=O)CC/C=C/c1ccc(OC)c(OC)c1</smiles>

ethyl (E)-5-(3,4-dimethoxyphenyl)-2,2-difluoropent-4-enoate (3aa)

Following the General Procedure at a $0.2 \mathrm{mmol}$ scale with alkene to afford the tittle compound 3aa as a colorless oil with $72 \%$ yield $(43.2 \mathrm{mg})$.

${ }^{1}$ H NMR $\left(500 \mathrm{MHz}, \mathrm{CDCl}_{3}\right) \delta 6.90-6.88(\mathrm{~m}, 2 \mathrm{H}), 6.81(\mathrm{~d}, J=8.0 \mathrm{~Hz}, 1 \mathrm{H}), 6.49(\mathrm{~d}, J=16.0 \mathrm{~Hz}, 1 \mathrm{H})$, $5.98-5.92(\mathrm{~m}, 1 \mathrm{H}), 4.32$ (q, $J=7.5 \mathrm{~Hz}, 2 \mathrm{H}), 3.90$ (s, 3H), $3.88(\mathrm{~s}, 3 \mathrm{H}), 3.01-2.93(\mathrm{~m}, 2 \mathrm{H}), 1.32(\mathrm{t}, J$ $=7.0 \mathrm{~Hz}, 3 \mathrm{H})$.

${ }^{13} \mathbf{C}$ NMR $\left(126 \mathrm{MHz}, \mathrm{CDCl}_{3}\right) \delta 163.9(\mathrm{t}, J=33.4 \mathrm{~Hz}), 149.1,149.0,136.1,129.5,119.7,115.8(\mathrm{t}, J=5.8$ $\mathrm{Hz}), 115.4(\mathrm{t}, J=252.3 \mathrm{~Hz}), 111.0,108.7,62.8,55.9,55.8,38.5(\mathrm{t}, J=23.8 \mathrm{~Hz}), 14.0$.

${ }^{19} \mathbf{F}$ NMR $\left(471 \mathrm{MHz}, \mathrm{CDCl}_{3}\right) \delta-105.2(\mathrm{t}, J=15.5 \mathrm{~Hz})$.

HRMS (ESI-TOF) (m/z): calcd for $\mathrm{C}_{15} \mathrm{H}_{18} \mathrm{~F}_{2} \mathrm{NaO}_{4}{ }^{+}\left([\mathrm{M}+\mathrm{Na}]^{+}\right), 323.1065$; found, 323.1068 . 
<smiles>CCOC(=O)CC/C=C/c1ccc(OS(C)(=O)=O)c(OC)c1</smiles>

ethyl (E)-5-(4-((tert-butyldimethylsilyl)oxy)-3-methoxyphenyl)-2,2-difluoropent-4-enoate

(3ab)

Following the General Procedure at a $0.2 \mathrm{mmol}$ scale with alkene to afford the tittle compound 3ab as a colorless oil with $65 \%$ yield $(52.0 \mathrm{mg})$.

${ }^{1} \mathrm{H}$ NMR $\left(500 \mathrm{MHz}, \mathrm{CDCl}_{3}\right) \delta 6.86(\mathrm{~d}, J=2.0 \mathrm{~Hz}, 1 \mathrm{H}), 6.82-6.75(\mathrm{~m}, 2 \mathrm{H}), 6.47(\mathrm{~d}, J=15.5 \mathrm{~Hz}, 1 \mathrm{H})$, $5.97-5.91(\mathrm{~m}, 1 \mathrm{H}), 4.32(\mathrm{q}, J=7.0 \mathrm{~Hz}, 2 \mathrm{H}), 3.81(\mathrm{~s}, 3 \mathrm{H}), 3.00-2.92(\mathrm{~m}, 2 \mathrm{H}), 1.32(\mathrm{t}, J=7.0 \mathrm{~Hz}, 3 \mathrm{H})$, 0.99 (s, 9H), 0.15 (s, 6H).

${ }^{13} \mathbf{C}$ NMR $\left(151 \mathrm{MHz}, \mathrm{CDCl}_{3}\right) \delta 163.9(\mathrm{t}, J=32.5 \mathrm{~Hz}), 151.0,145.3,136.3,130.4,120.9,119.6,115.8(\mathrm{t}$, $J=5.4 \mathrm{~Hz}), 115.4(\mathrm{t}, J=251.7 \mathrm{~Hz}), 109.7,62.8,55.5,38.5(\mathrm{t}, J=23.7 \mathrm{~Hz}), 25.7,18.5,14.0,-4.7$.

${ }^{19} \mathbf{F}$ NMR $\left(471 \mathrm{MHz}, \mathrm{CDCl}_{3}\right) \delta-105.3(\mathrm{t}, J=16.5 \mathrm{~Hz})$.

HRMS (ESI-TOF) (m/z): calcd for $\mathrm{C}_{20} \mathrm{H}_{30} \mathrm{~F}_{2} \mathrm{NaO}_{4} \mathrm{Si}^{+}\left([\mathrm{M}+\mathrm{Na}]^{+}\right)$, 423.1774; found, 423.1785 .<smiles>CCOC(=O)C(F)(F)CC=Cc1ccc(Cl)cc1</smiles>

ethyl (E)-5-(4-chlorophenyl)-2,2-difluoropent-4-enoate (3ac)

Following the General Procedure at a $0.2 \mathrm{mmol}$ scale with alkene to afford the tittle compound 3ac as a colorless oil with $68 \%$ yield $(37.3 \mathrm{mg})$.

${ }^{1} \mathbf{H}$ NMR $\left(600 \mathrm{MHz}, \mathrm{CDCl}_{3}\right) \delta 7.28(\mathrm{~s}, 4 \mathrm{H}), 6.52(\mathrm{~d}, J=15.6 \mathrm{~Hz}, 1 \mathrm{H}), 6.10-6.05(\mathrm{~m}, 1 \mathrm{H}), 4.32(\mathrm{q}, J=$ $7.2 \mathrm{~Hz}, 2 \mathrm{H}), 3.02-2.95(\mathrm{~m}, 2 \mathrm{H}), 1.32(\mathrm{t}, J=7.2 \mathrm{~Hz}, 3 \mathrm{H})$.

${ }^{13} \mathbf{C}$ NMR $\left(126 \mathrm{MHz}, \mathrm{CDCl}_{3}\right) \delta 163.8(\mathrm{t}, J=32.6 \mathrm{~Hz}), 135.2,134.9,133.6,128.8,127.6,118.8(\mathrm{t}, J=$ $5.7 \mathrm{~Hz}), 115.2(\mathrm{t}, J=252.3 \mathrm{~Hz}), 62.9,38.4(\mathrm{t}, J=23.9 \mathrm{~Hz}), 14.0$.

${ }^{19} \mathbf{F}$ NMR $\left(471 \mathrm{MHz}, \mathrm{CDCl}_{3}\right) \delta-105.2(\mathrm{t}, J=16.5 \mathrm{~Hz})$.

HRMS (ESI-TOF) (m/z): calcd for $\mathrm{C}_{13} \mathrm{H}_{13} \mathrm{ClF}_{2} \mathrm{NaO}_{2}{ }^{+}\left([\mathrm{M}+\mathrm{Na}]^{+}\right)$, 297.0464; found, 297.0468 .<smiles>CCOC(=O)C(F)(F)F</smiles>

\section{ethyl (E)-5-([1,1'-biphenyl]-4-yl)-2,2-difluoropent-4-enoate (3ad)}

Following the General Procedure at a $0.2 \mathrm{mmol}$ scale with alkene to afford the tittle compound 3ad as a white solid with $70 \%$ yield $(44.3 \mathrm{mg}) . \mathrm{Mp}: 91-92{ }^{\circ} \mathrm{C}$

${ }^{1} \mathbf{H}$ NMR $\left(600 \mathrm{MHz}, \mathrm{CDCl}_{3}\right) \delta 7.59(\mathrm{~d}, J=7.8 \mathrm{~Hz}, 2 \mathrm{H}), 7.55(\mathrm{~d}, J=7.8 \mathrm{~Hz}, 2 \mathrm{H}), 7.45-7.42(\mathrm{~m}, 4 \mathrm{H})$, $7.34(\mathrm{t}, J=7.2 \mathrm{~Hz}, 1 \mathrm{H}), 6.60$ (d, $J=15.6 \mathrm{~Hz}, 1 \mathrm{H}), 6.16-6.11(\mathrm{~m}, 1 \mathrm{H}), 4.33$ (q, $J=7.2 \mathrm{~Hz}, 2 \mathrm{H}), 3.05-$ $2.98(\mathrm{~m}, 2 \mathrm{H}), 1.33(\mathrm{t}, J=7.2 \mathrm{~Hz}, 3 \mathrm{H})$.

${ }^{13} \mathrm{C}$ NMR $\left(151 \mathrm{MHz}, \mathrm{CDCl}_{3}\right) \delta 163.9(\mathrm{t}, J=32.8 \mathrm{~Hz}), 140.7,140.6,136.0,135.4,128.8,127.4,127.3$, 126.9, 126.8, $118.1(\mathrm{t}, J=5.4 \mathrm{~Hz}), 115.3(\mathrm{t}, J=251.6 \mathrm{~Hz}), 62.9,38.6(\mathrm{t}, J=24.2 \mathrm{~Hz}), 14.0$.

${ }^{19} \mathbf{F}$ NMR $\left(471 \mathrm{MHz}, \mathrm{CDCl}_{3}\right) \delta-105.1(\mathrm{t}, J=15.5 \mathrm{~Hz})$.

HRMS (ESI-TOF) $(\mathrm{m} / \mathrm{z})$ : calcd for $\mathrm{C}_{19} \mathrm{H}_{18} \mathrm{~F}_{2} \mathrm{NaO}_{2}{ }^{+}\left([\mathrm{M}+\mathrm{Na}]^{+}\right)$, 339.1167; found, 339.1171 . 
$\mathrm{Ph} \sim \mathrm{H}_{3} \sim \mathrm{CF}_{2} \mathrm{CO}_{2} \mathrm{Et}$

ethyl (E)-2,2-difluoro-8-phenyloct-7-enoate (3ae)

Following the General Procedure at a $0.2 \mathrm{mmol}$ scale with alkene to afford the tittle compound 3ae as a colorless oil with $56 \%$ yield $(31.6 \mathrm{mg})$.

${ }^{1} \mathbf{H}$ NMR $\left(600 \mathrm{MHz}, \mathrm{CDCl}_{3}\right) \delta 7.34-7.32(\mathrm{~m}, 2 \mathrm{H}), 7.29(\mathrm{t}, J=7.8 \mathrm{~Hz}, 2 \mathrm{H}), 7.21-7.18(\mathrm{~m}, 1 \mathrm{H}), 6.38$ $(\mathrm{d}, J=15.6 \mathrm{~Hz}, 1 \mathrm{H}), 6.21-6.16(\mathrm{~m}, 1 \mathrm{H}), 4.31(\mathrm{q}, J=7.2 \mathrm{~Hz}, 2 \mathrm{H}), 2.25-2.22(\mathrm{~m}, 2 \mathrm{H}), 2.13-2.05(\mathrm{~m}$, $2 \mathrm{H}), 1.55-1.53(\mathrm{~m}, 4 \mathrm{H}), 1.33(\mathrm{t}, J=7.2 \mathrm{~Hz}, 3 \mathrm{H})$.

${ }^{13}$ C NMR $\left(126 \mathrm{MHz}, \mathrm{CDCl}_{3}\right) \delta 164.4(\mathrm{t}, J=32.8 \mathrm{~Hz}), 137.6,130.4,129.9,128.5,126.9,125.9,116.3$ (t, $J=250.4 \mathrm{~Hz}), 62.7,34.3(\mathrm{t}, J=23.1 \mathrm{~Hz}), 32.6,28.7,21.0$ (t, $J=4.7 \mathrm{~Hz}), 13.9$.

${ }^{19} \mathbf{F}$ NMR $\left(471 \mathrm{MHz}, \mathrm{CDCl}_{3}\right) \delta-105.9(\mathrm{t}, J=16.5 \mathrm{~Hz})$.

HRMS (ESI-TOF) (m/z): calcd for $\mathrm{C}_{16} \mathrm{H}_{20} \mathrm{~F}_{2} \mathrm{NaO}_{2}{ }^{+}\left([\mathrm{M}+\mathrm{Na}]^{+}\right)$, 305.1324; found, 305.1307 .

$\mathrm{Ph} \curvearrowright \mathrm{H}_{4} \sim \mathrm{CF}_{2} \mathrm{CO}_{2} \mathrm{Et}$

ethyl (E)-2,2-difluoro-9-phenylnon-8-enoate (3af)

Following the General Procedure at a $0.2 \mathrm{mmol}$ scale with alkene to afford the tittle compound 3af as a colorless oil with $49 \%$ yield $(29.0 \mathrm{mg})$.

${ }^{1} \mathbf{H}$ NMR $\left(600 \mathrm{MHz}, \mathrm{CDCl}_{3}\right) \delta 7.33(\mathrm{~d}, J=6.6 \mathrm{~Hz}, 2 \mathrm{H}), 7.30-7.27(\mathrm{~m}, 2 \mathrm{H}), 7.20-7.18(\mathrm{~m}, 1 \mathrm{H}), 6.37$ (d, $J=15.6 \mathrm{~Hz}, 1 \mathrm{H}), 6.22-6.18(\mathrm{~m}, 1 \mathrm{H}), 4.32(\mathrm{q}, J=7.2 \mathrm{~Hz}, 2 \mathrm{H}), 2.23-2.19(\mathrm{~m}, 2 \mathrm{H}), 2.10-2.02(\mathrm{~m}$, 2H), $1.53-1.47$ (m, 4H), $1.44-1.39(\mathrm{~m}, 2 \mathrm{H}), 1.35(\mathrm{t}, J=7.2 \mathrm{~Hz}, 3 \mathrm{H})$.

${ }^{13}$ C NMR $\left(126 \mathrm{MHz}, \mathrm{CDCl}_{3}\right) \delta 164.4(\mathrm{t}, J=33.4 \mathrm{~Hz}), 137.7,130.5,130.1,128.5,126.9,125.9,116.3(\mathrm{t}$, $J=250.4 \mathrm{~Hz}), 62.7,34.4(\mathrm{t}, J=23.7 \mathrm{~Hz}), 32.7,28.9,28.6,21.3(\mathrm{t}, J=3.9 \mathrm{~Hz}), 14.0$.

${ }^{19} \mathbf{F}$ NMR $\left(471 \mathrm{MHz}, \mathrm{CDCl}_{3}\right) \delta-105.9(\mathrm{t}, J=16.5 \mathrm{~Hz})$.

HRMS (ESI-TOF) (m/z): calcd for $\mathrm{C}_{17} \mathrm{H}_{22} \mathrm{~F}_{2} \mathrm{NaO}_{2}{ }^{+}\left([\mathrm{M}+\mathrm{Na}]^{+}\right), 319.1480$; found, 319.1488 .

$\mathrm{Ph} \curvearrowright \mathrm{H}_{5}-\mathrm{CF}_{2} \mathrm{CO}_{2} \mathrm{Et}$

ethyl (E)-2,2-difluoro-10-phenyldec-9-enoate (3ag)

Following the General Procedure at a $0.2 \mathrm{mmol}$ scale with alkene to afford the tittle compound 3ag as a colorless oil with $39 \%$ yield $(24.2 \mathrm{mg})$.

${ }^{1} \mathbf{H}$ NMR $\left(600 \mathrm{MHz}, \mathrm{CDCl}_{3}\right) \delta 7.33(\mathrm{~d}, J=7.8 \mathrm{~Hz}, 2 \mathrm{H}), 7.28(\mathrm{t}, J=7.2 \mathrm{~Hz}, 2 \mathrm{H}), 7.19(\mathrm{~d}, J=7.2 \mathrm{~Hz}$, $1 \mathrm{H}), 6.37(\mathrm{~d}, J=15.6 \mathrm{~Hz}, 1 \mathrm{H}), 6.23-6.18(\mathrm{~m}, 1 \mathrm{H}), 4.32(\mathrm{q}, J=7.2 \mathrm{~Hz}, 2 \mathrm{H}), 2.22-2.19(\mathrm{~m}, 2 \mathrm{H}), 2.09$ $-2.01(\mathrm{~m}, 2 \mathrm{H}), 1.49-1.45(\mathrm{~m}, 4 \mathrm{H}), 1.38-1.33(\mathrm{~m}, 7 \mathrm{H})$.

${ }^{13} \mathbf{C}$ NMR $\left(151 \mathrm{MHz}, \mathrm{CDCl}_{3}\right) \delta 164.4(\mathrm{t}, J=32.9 \mathrm{~Hz}), 137.8,130.8,129.9,128.5,126.8,125.9,116.4(\mathrm{t}$, $J=249.8 \mathrm{~Hz}), 62.7,34.5(\mathrm{t}, J=23.1 \mathrm{~Hz}), 32.9,29.1,28.9,28.8,21.4(\mathrm{t}, J=4.1 \mathrm{~Hz}), 14.0$.

${ }^{19} \mathbf{F}$ NMR $\left(471 \mathrm{MHz}, \mathrm{CDCl}_{3}\right) \delta-105.9(\mathrm{t}, J=17.0 \mathrm{~Hz})$.

HRMS (ESI-TOF) $(\mathrm{m} / \mathrm{z})$ : calcd for $\mathrm{C}_{18} \mathrm{H}_{24} \mathrm{~F}_{2} \mathrm{NaO}_{2}{ }^{+}\left([\mathrm{M}+\mathrm{Na}]^{+}\right)$, 333.1637; found, 333.1634.<smiles>CCOC(=O)[C-]CC=CC=Cc1ccccc1</smiles>

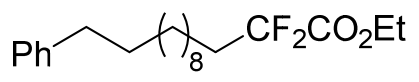

ethyl (E)-2,2-difluoro-13-phenyltridec-12-enoate (3ah) and ethyl 2,2-difluoro-13phenyltridecanoate (3ah')

The products 3ah and byproduct 3ah' could not be readily separated by silica gel chromatography, so 
they were characterized as a mixture. The ${ }^{1} \mathrm{H}$ NMR Spectroscopy showed a mixture of $\mathbf{3 a h}$ and $\mathbf{3 a h}$ ' (3ah:3ah' $=1: 1)$.

${ }^{1} \mathbf{H}$ NMR $\left(500 \mathrm{MHz}, \mathrm{CDCl}_{3}\right) \delta 7.33(\mathrm{~d}, J=7.0 \mathrm{~Hz}, 2 \mathrm{H}), 7.29-7.24(\mathrm{~m}, 4 \mathrm{H}), 7.19-7.16(\mathrm{~m}, 4 \mathrm{H}), 6.37$ (d, $J=16.0 \mathrm{~Hz}, 1 \mathrm{H}), 6.25-6.19(\mathrm{~m}, 1 \mathrm{H}), 4.33-4.29$ (m, 4H), 2.59 (t, $J=7.5 \mathrm{~Hz}, 2 \mathrm{H}), 2.20$ (q, $J=7.5$ $\mathrm{Hz}, 2 \mathrm{H}), 2.10-1.94(\mathrm{~m}, 6 \mathrm{H}), 1.62-1.58(\mathrm{~m}, 2 \mathrm{H}), 1.47-1.44(\mathrm{~m}, 6 \mathrm{H}), 1.36-1.26(\mathrm{~m}, 28 \mathrm{H})$.

HRMS (ESI-TOF) (m/z): calcd for $\mathrm{C}_{21} \mathrm{H}_{30} \mathrm{~F}_{2} \mathrm{NaO}_{2}{ }^{+}\left([\mathrm{M}+\mathrm{Na}]^{+}\right)$, 375.2106; found, 375.2105 .

PhthN $\mathrm{CF}_{2} \mathrm{CO}_{2} \mathrm{Et}$

ethyl (E)-5-(1,3-dioxoisoindolin-2-yl)-2,2-difluoropent-4-enoate (3ai)

Following the General Procedure at a $0.2 \mathrm{mmol}$ scale with alkene to afford the tittle compound 3ai as a white solid with $36 \%$ yield $(22.2 \mathrm{mg})$. Mp: $115-116^{\circ} \mathrm{C}$.

${ }^{1} \mathbf{H}$ NMR $\left(500 \mathrm{MHz}, \mathrm{CDCl}_{3}\right) \delta 7.89-7.85(\mathrm{~m}, 2 \mathrm{H}), 7.78-7.74(\mathrm{~m}, 2 \mathrm{H}), 6.82(\mathrm{~d}, J=14.5 \mathrm{~Hz}, 1 \mathrm{H}), 6.61$ -6.55 (m, 1H), 4.35 (q, $J=7.0 \mathrm{~Hz}, 2 \mathrm{H}), 2.98-2.90(\mathrm{~m}, 2 \mathrm{H}), 1.36$ (t, $J=7.2 \mathrm{~Hz}, 3 \mathrm{H})$.

${ }^{13} \mathbf{C}$ NMR $\left(151 \mathrm{MHz}, \mathrm{CDCl}_{3}\right) \delta 166.1,163.7(\mathrm{t}, J=32.6 \mathrm{~Hz}), 134.6,131.5,123.7,122.9,115.0(\mathrm{t}, J=$ $251.7 \mathrm{~Hz}), 109.4(\mathrm{t}, J=5.7 \mathrm{~Hz}), 63.0,36.9(\mathrm{t}, J=24.5 \mathrm{~Hz}), 14.0$.

${ }^{19} \mathbf{F}$ NMR $\left(471 \mathrm{MHz}, \mathrm{CDCl}_{3}\right) \delta-105.3(\mathrm{t}, J=15.5 \mathrm{~Hz})$.

HRMS (ESI-TOF) (m/z): calcd for $\mathrm{C}_{15} \mathrm{H}_{13} \mathrm{~F}_{2} \mathrm{NNaO}_{4}{ }^{+}\left([\mathrm{M}+\mathrm{Na}]^{+}\right)$, 332.0705; found, 332.0711 .<smiles>CCOC(=O)C(F)(F)CCC/C=C/C(=O)N(C)c1ccccc1</smiles>

ethyl (E)-2,2-difluoro-7-(methyl(phenyl)amino)-7-oxohept-5-enoate (3aj)

The products 3 aj and other indistinguishable byproduct could not be readily separated by silica gel chromatography, so it was characterized as a mixture.

${ }^{1} \mathbf{H}$ NMR $\left(500 \mathrm{MHz}, \mathrm{CDCl}_{3}\right) \delta 7.42-7.41(\mathrm{~m}, 2 \mathrm{H}), 7.36-7.33(\mathrm{~m}, 1 \mathrm{H}), 7.20-7.16(\mathrm{~m}, 2 \mathrm{H}), 6.86-$ $6.81(\mathrm{~m}, 1 \mathrm{H}), 5.79(\mathrm{~d}, J=15.0 \mathrm{~Hz}, 1 \mathrm{H}), 4.30(\mathrm{~d}, J=7.0 \mathrm{~Hz}, 2 \mathrm{H}), 3.34(\mathrm{~s}, 3 \mathrm{H}), 2.29-2.24(\mathrm{~m}, 2 \mathrm{H}), 2.14$ $-2.05(\mathrm{~m}, 2 \mathrm{H}), 1.33(\mathrm{~d}, J=7.0 \mathrm{~Hz}, 3 \mathrm{H})$.

HRMS (ESI-TOF) (m/z): calcd for $\mathrm{C}_{16} \mathrm{H}_{19} \mathrm{~F}_{2} \mathrm{NNaO}_{3}{ }^{+}\left([\mathrm{M}+\mathrm{Na}]^{+}\right)$, 334.1225; found, 334.1221 .

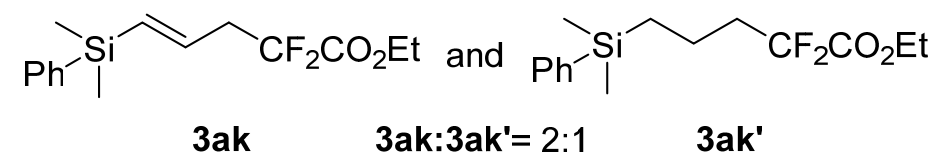

The products 3ak and byproduct 3ak' could not be readily separated by silica gel chromatography, so they were characterized as a mixture. The ${ }^{1} \mathrm{H}$ NMR Spectroscopy showed a mixture of 3ak and 3ak' (3ak:3ak' $=2: 1)$.

${ }^{1} \mathbf{H}$ NMR $\left(500 \mathrm{MHz}, \mathrm{CDCl}_{3}\right) \delta 7.53-7.47(\mathrm{~m}, 3 \mathrm{H}), 7.36-7.35(\mathrm{~m}, 5 \mathrm{H}), 6.07-5.96(\mathrm{~m}, 2 \mathrm{H}), 4.31-$ $4.25(\mathrm{~m}, 3 \mathrm{H}), 2.96-2.76(\mathrm{~m}, 2 \mathrm{H}), 2.11-2.01(\mathrm{~m}, 1 \mathrm{H}), 1.32-1.26(\mathrm{~m}, 5 \mathrm{H}), 0.82-0.74(\mathrm{~m}, 1 \mathrm{H}), 0.34$ (s, 6H), $0.27(\mathrm{~s}, 3 \mathrm{H})$.

HRMS (ESI-TOF) (m/z): calcd for $\mathrm{C}_{15} \mathrm{H}_{20} \mathrm{~F}_{2} \mathrm{NaO}_{2} \mathrm{Si}^{+}\left([\mathrm{M}+\mathrm{Na}]^{+}\right)$, 321.1093; found, 321.1088 . 
<smiles>COC(=O)C(F)(F)CC/C=C/c1ccccc1</smiles>

methyl (E)-2,2-difluoro-6-phenylhex-5-enoate (3al)

Following the General Procedure at a $0.2 \mathrm{mmol}$ scale with alkene to afford the tittle compound 3al as a colorless oil with $76 \%$ yield $(36.5 \mathrm{mg})$.

${ }^{1} \mathbf{H}$ NMR $\left(500 \mathrm{MHz}, \mathrm{CDCl}_{3}\right) \delta 7.34-7.32(\mathrm{~m}, 2 \mathrm{H}), 7.31-7.28(\mathrm{~m}, 2 \mathrm{H}), 7.23-7.20(\mathrm{~m}, 1 \mathrm{H}), 6.43(\mathrm{~d}, J$ $=15.5 \mathrm{~Hz}, 1 \mathrm{H}), 6.18-6.12(\mathrm{~m}, 1 \mathrm{H}), 3.84(\mathrm{~s}, 3 \mathrm{H}), 2.44-2.40(\mathrm{~m}, 2 \mathrm{H}), 2.31-2.23(\mathrm{~m}, 2 \mathrm{H})$.

${ }^{13} \mathrm{C}$ NMR $\left(151 \mathrm{MHz}, \mathrm{CDCl}_{3}\right) \delta 164.7(\mathrm{t}, J=32.9 \mathrm{~Hz}), 137.1,131.5,128.5,127.3,126.0,116.0(\mathrm{t}, J=$ $250.7 \mathrm{~Hz}), 53.3,34.3(\mathrm{t}, J=23.1 \mathrm{~Hz}), 25.1(\mathrm{t}, J=4.8 \mathrm{~Hz})$.

${ }^{19} \mathbf{F}$ NMR $\left(471 \mathrm{MHz}, \mathrm{CDCl}_{3}\right) \delta-105.8(\mathrm{t}, J=16.5 \mathrm{~Hz})$.

HRMS (ESI-TOF) (m/z): calcd for $\mathrm{C}_{13} \mathrm{H}_{14} \mathrm{~F}_{2} \mathrm{NaO}_{2}{ }^{+}\left([\mathrm{M}+\mathrm{Na}]^{+}\right)$, 263.0854; found, 263.0850 .<smiles>O=C(OCc1ccccc1)C(F)(F)CC/C=C/c1ccccc1</smiles>

benzyl (E)-2,2-difluoro-6-phenylhex-5-enoate (3am)

Following the General Procedure at a $0.2 \mathrm{mmol}$ scale with alkene to afford the tittle compound 3am as a colorless oil with $63 \%$ yield (39.8 $\mathrm{mg}$ ).

${ }^{1}$ H NMR $\left(500 \mathrm{MHz}, \mathrm{CDCl}_{3}\right) \delta 7.39-7.34(\mathrm{~m}, 5 \mathrm{H}), 7.32-7.28(\mathrm{~m}, 4 \mathrm{H}), 7.23-7.19(\mathrm{~m}, 1 \mathrm{H}), 6.38(\mathrm{~d}, J$ $=16.0 \mathrm{~Hz}, 1 \mathrm{H}), 6.15-6.09(\mathrm{~m}, 1 \mathrm{H}), 5.24(\mathrm{~s}, 2 \mathrm{H}), 2.40-2.35(\mathrm{~m}, 2 \mathrm{H}), 2.30-2.20(\mathrm{~m}, 2 \mathrm{H})$.

${ }^{13}$ C NMR $\left(151 \mathrm{MHz}, \mathrm{CDCl}_{3}\right) \delta 164.0(\mathrm{t}, J=32.9 \mathrm{~Hz}), 137.1,134.3,131.4,128.8,128.7,128.5,128.4$, 127.3, 126.0, $115.9(\mathrm{t}, J=250.8 \mathrm{~Hz}), 68.3,34.3(\mathrm{t}, J=23.0 \mathrm{~Hz}), 25.1(\mathrm{t}, J=4.8 \mathrm{~Hz})$.

${ }^{19} \mathbf{F}$ NMR $\left(471 \mathrm{MHz}, \mathrm{CDCl}_{3}\right) \delta-105.8(\mathrm{t}, J=16.0 \mathrm{~Hz})$.

HRMS (ESI-TOF) $(\mathrm{m} / \mathrm{z})$ : calcd for $\mathrm{C}_{19} \mathrm{H}_{18} \mathrm{~F}_{2} \mathrm{NaO}_{2}{ }^{+}\left([\mathrm{M}+\mathrm{Na}]^{+}\right)$, 339.1167; found, 339.1166 .<smiles>CCCc1ccc(COC(=O)C(F)(F)CC/C=C/c2ccccc2)cc1</smiles>

\section{4-isopropylbenzyl (E)-2,2-difluoro-6-phenylhex-5-enoate (3an)}

Following the General Procedure at a $0.2 \mathrm{mmol}$ scale with alkene to afford the tittle compound 3an as a colorless oil with $50 \%$ yield $(35.8 \mathrm{mg})$.

${ }^{1} \mathbf{H}$ NMR $\left(500 \mathrm{MHz}, \mathrm{CDCl}_{3}\right) \delta 7.32-7.27(\mathrm{~m}, 6 \mathrm{H}), 7.25-7.21(\mathrm{~m}, 3 \mathrm{H}), 6.39(\mathrm{~d}, J=15.5 \mathrm{~Hz}, 1 \mathrm{H}), 6.15$ $-6.09(\mathrm{~m}, 1 \mathrm{H}), 5.21(\mathrm{~s}, 2 \mathrm{H}), 2.94-2.89(\mathrm{~m}, 1 \mathrm{H}), 2.39-2.35(\mathrm{~m}, 2 \mathrm{H}), 2.29-2.20(\mathrm{~m}, 2 \mathrm{H}), 1.25(\mathrm{~d}, J=$ $7.0 \mathrm{~Hz}, 6 \mathrm{H})$.

${ }^{13}$ C NMR $\left(151 \mathrm{MHz}, \mathrm{CDCl}_{3}\right) \delta 164.1(\mathrm{t}, J=32.9 \mathrm{~Hz}), 149.7,137.1,131.6,131.4,128.7,128.5,127.4$, 127.3, 126.8, 126.0, 115.9 (t, $J=250.8 \mathrm{~Hz}), 68.3,34.3(\mathrm{t}, J=23.1 \mathrm{~Hz}), 33.9,25.1(\mathrm{t}, J=4.8 \mathrm{~Hz}), 23.9$.

${ }^{19} \mathbf{F}$ NMR $\left(471 \mathrm{MHz}, \mathrm{CDCl}_{3}\right) \delta-105.8(\mathrm{t}, J=17.0 \mathrm{~Hz})$.

HRMS (ESI-TOF) (m/z): calcd for $\mathrm{C}_{22} \mathrm{H}_{24} \mathrm{~F}_{2} \mathrm{NaO}_{2}{ }^{+}\left([\mathrm{M}+\mathrm{Na}]^{+}\right)$, 381.1637; found, 381.1644. 
<smiles>O=C(OCc1ccc(F)cc1)C(F)(F)CC/C=C/c1ccccc1</smiles>

\section{4-fluorobenzyl (E)-2,2-difluoro-6-phenylhex-5-enoate (3ao)}

Following the General Procedure at a $0.2 \mathrm{mmol}$ scale with alkene to afford the tittle compound 3ao as a colorless oil with $52 \%$ yield $(34.7 \mathrm{mg})$.

${ }^{1} \mathbf{H}$ NMR $\left(500 \mathrm{MHz}, \mathrm{CDCl}_{3}\right) \delta 7.34-7.28(\mathrm{~m}, 6 \mathrm{H}), 7.23-7.20(\mathrm{~m}, 1 \mathrm{H}), 7.06(\mathrm{t}, J=8.5 \mathrm{~Hz}, 2 \mathrm{H}), 6.38$ (d, $J=16.0 \mathrm{~Hz}, 1 \mathrm{H}), 6.15-6.09$ (m, 1H), 5.20 (s, 2H), $2.39-2.35(\mathrm{~m}, 2 \mathrm{H}), 2.29-2.20$ (m, 2H).

${ }^{13}$ C NMR $\left(151 \mathrm{MHz}, \mathrm{CDCl}_{3}\right) \delta 164.0(\mathrm{t}, J=33.2 \mathrm{~Hz}), 163.0(\mathrm{~d}, J=247.9 \mathrm{~Hz}), 137.0,131.5,130.6(\mathrm{~d}, J$ $=8.5 \mathrm{~Hz}), 130.1(\mathrm{~d}, J=3.3 \mathrm{~Hz}), 128.6,127.4,127.2,126.0,115.9(\mathrm{~d}, J=251.0 \mathrm{~Hz}), 115.7(\mathrm{~d}, J=21.9$ $\mathrm{Hz}), 67.5,34.3(\mathrm{~d}, J=23.1 \mathrm{~Hz}), 25.1(\mathrm{~d}, J=4.8 \mathrm{~Hz})$.

${ }^{19}$ F NMR $\left(471 \mathrm{MHz}, \mathrm{CDCl}_{3}\right) \delta-105.8(\mathrm{~d}, J=17.0 \mathrm{~Hz}),(-112.4)-(-112.5)(\mathrm{m})$.

HRMS (ESI-TOF) (m/z): calcd for $\mathrm{C}_{19} \mathrm{H}_{17} \mathrm{~F}_{3} \mathrm{NaO}_{2}{ }^{+}\left([\mathrm{M}+\mathrm{Na}]{ }^{+}\right)$, 357.1073; found, 357.1054.<smiles>O=C(N1CCOCC1)C(F)(F)CC/C=C/c1ccccc1</smiles>

(E)-2,2-difluoro-1-morpholino-6-phenylhex-5-en-1-one (3ap)

Following the General Procedure at a $0.2 \mathrm{mmol}$ scale with alkene to afford the tittle compound 3ap as a colorless oil with $43 \%$ yield $(25.4 \mathrm{mg})$.

${ }^{1}$ H NMR $\left(500 \mathrm{MHz}, \mathrm{CDCl}_{3}\right) \delta 7.35-7.33(\mathrm{~m}, 2 \mathrm{H}), 7.31-7.28(\mathrm{~m}, 2 \mathrm{H}), 7.23-7.19(\mathrm{~m}, 1 \mathrm{H}), 6.45(\mathrm{~d}, J$ $=16.0 \mathrm{~Hz}, 1 \mathrm{H}), 6.25-6.19(\mathrm{~m}, 1 \mathrm{H}), 3.77-3.75(\mathrm{~m}, 2 \mathrm{H}), 3.73-3.70(\mathrm{~m}, 4 \mathrm{H}), 3.66-3.64(\mathrm{~m}, 2 \mathrm{H}), 2.50$ $-2.45(\mathrm{~m}, 2 \mathrm{H}), 2.39-2.28(\mathrm{~m}, 2 \mathrm{H})$.

${ }^{13}$ C NMR $\left(151 \mathrm{MHz}, \mathrm{CDCl}_{3}\right) \delta 161.9(\mathrm{t}, J=29.4 \mathrm{~Hz}), 137.4,130.9,128.5,128.4,127.1,126.0,119.3(\mathrm{t}$, $J=254.4 \mathrm{~Hz}), 66.8,66.7,46.5(\mathrm{t}, J=6.3 \mathrm{~Hz}), 43.3,34.4(\mathrm{t}, J=22.7 \mathrm{~Hz}), 25.2(\mathrm{t}, J=5.1 \mathrm{~Hz})$.

${ }^{19}$ F NMR $\left(565 \mathrm{MHz}, \mathrm{CDCl}_{3}\right) \delta-99.5(\mathrm{t}, J=18.6 \mathrm{~Hz})$.

HRMS (ESI-TOF) (m/z): calcd for $\mathrm{C}_{16} \mathrm{H}_{19} \mathrm{~F}_{2} \mathrm{NNaO}_{2}{ }^{+}\left([\mathrm{M}+\mathrm{Na}]^{+}\right)$, 318.1267; found, 318.1278.<smiles>O=C(Nc1ccccc1)C(F)(F)CC/C=C/c1ccccc1</smiles>

\section{(E)-2,2-difluoro-N,6-diphenylhex-5-enamide(3aq)}

Following the General Procedure at a $0.2 \mathrm{mmol}$ scale with alkene to afford the tittle compound 3aq as a white solid with $36 \%$ yield $(21.7 \mathrm{mg})$. Mp: $86-87^{\circ} \mathrm{C}$

${ }^{1} \mathbf{H}$ NMR $\left(600 \mathrm{MHz}, \mathrm{CDCl}_{3}\right) \delta 7.96(\mathrm{~s}, 1 \mathrm{H}), 7.56-7.54(\mathrm{~m}, 2 \mathrm{H}), 7.36(\mathrm{t}, J=7.8 \mathrm{~Hz}, 2 \mathrm{H}), 7.32-7.26$ (m, 4H), $7.21-7.18(\mathrm{~m}, 2 \mathrm{H}), 6.44(\mathrm{~d}, J=15.6 \mathrm{~Hz}, 1 \mathrm{H}), 6.21-6.16(\mathrm{~m}, 1 \mathrm{H}), 2.49-2.45(\mathrm{~m}, 2 \mathrm{H}), 2.43$ $-2.35(\mathrm{~m}, 2 \mathrm{H})$.

${ }^{13}$ C NMR $\left(151 \mathrm{MHz}, \mathrm{CDCl}_{3}\right) \delta 161.9(\mathrm{t}, J=28.7 \mathrm{~Hz}), 137.1,135.9,131.3,129.2,128.5,127.5,127.3$, 126.0, 125.6, 120.2, 118.0 (t, $J=254.3 \mathrm{~Hz}), 33.5(\mathrm{t}, J=22.8 \mathrm{~Hz}), 25.3(\mathrm{t}, J=4.7 \mathrm{~Hz})$.

${ }^{19} \mathbf{F}$ NMR $\left(471 \mathrm{MHz}, \mathrm{CDCl}_{3}\right) \delta-105.3(\mathrm{t}, J=17.9 \mathrm{~Hz})$.

HRMS (ESI-TOF) (m/z): calcd for $\mathrm{C}_{18} \mathrm{H}_{17} \mathrm{~F}_{2} \mathrm{NNaO}^{+}\left([\mathrm{M}+\mathrm{Na}]^{+}\right), 324.1170$; found, 324.1160. 


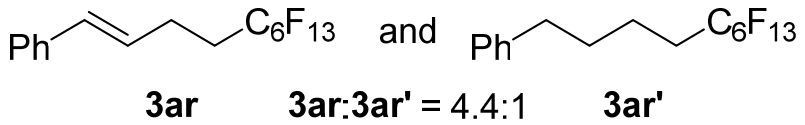

$(E)-\left(10,10,10,10,10,10,10,10,10,10,10,10,10-t r i d e c a f l u o r o-10 \lambda^{16}\right.$-deca-1-en-5,7,9-triyn-1yl)benzene (3ar) and $\left(10,10,10,10,10,10,10,10,10,10,10,10,10\right.$-tridecafluoro-10 $\lambda^{16}$-deca-5,7,9triyn-1-yl)benzene (3ar')

The products 3ar and byproduct 3ar' could not be readily separated by silica gel chromatography, so they were characterized as a mixture. The ${ }^{1} \mathrm{H}$ NMR Spectroscopy showed a mixture of 3ar and 3ar' (3ar:3ar' $=4.4: 1)$.

${ }^{1} \mathbf{H}$ NMR $\left(500 \mathrm{MHz}, \mathrm{CDCl}_{3}\right) \delta 7.35-7.29(\mathrm{~m}, 4 \mathrm{H}), 7.24-7.20(\mathrm{~m}, 1 \mathrm{H}), 6.48(\mathrm{~d}, J=16.0 \mathrm{~Hz}, 1 \mathrm{H}), 6.21$ $-6.15(\mathrm{~m}, 1 \mathrm{H}), 2.66-2.51(\mathrm{~m}, 2 \mathrm{H}), 2.29-2.20(\mathrm{~m}, 2 \mathrm{H})$.

${ }^{13} \mathrm{C}$ NMR $\left(151 \mathrm{MHz}, \mathrm{CDCl}_{3}\right) \delta 137.0,131.8,128.6,128.4,128.3,127.4,126.9,126.1,120.3-120.0$ (m), $118.4-117.9(\mathrm{~m}), 116.7-116.0(\mathrm{~m}), 112.9-107.8(\mathrm{~m}), 39.0,35.5,30.9$ (t, $J=22.0 \mathrm{~Hz}), 23.8$ (t, $J$ $=4.4 \mathrm{~Hz}), 19.8(\mathrm{t}, J=3.6 \mathrm{~Hz})$.

${ }^{19}$ F NMR $\left(471 \mathrm{MHz}, \mathrm{CDCl}_{3}\right) \delta-80.8-(-80.9)(\mathrm{m}),(-114.3)-(-114.5)(\mathrm{m}),(-121.88)-(-121.96)(\mathrm{m}),(-$ $122.8)-(-122.9)(\mathrm{m}),(-123.5)-(-123.6)(\mathrm{m}),(-126.1)-(-126.2)(\mathrm{m})$.

HRMS (ESI-TOF) $(\mathrm{m} / \mathrm{z})$ : calcd for $\mathrm{C}_{16} \mathrm{H}_{12} \mathrm{~F}_{12}{ }^{+}\left([\mathrm{M}+\mathrm{H}]^{+}\right)$, 451.0726; found, 451.0719.

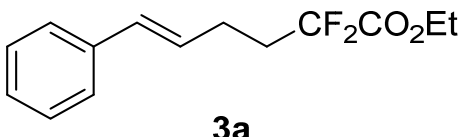

3a

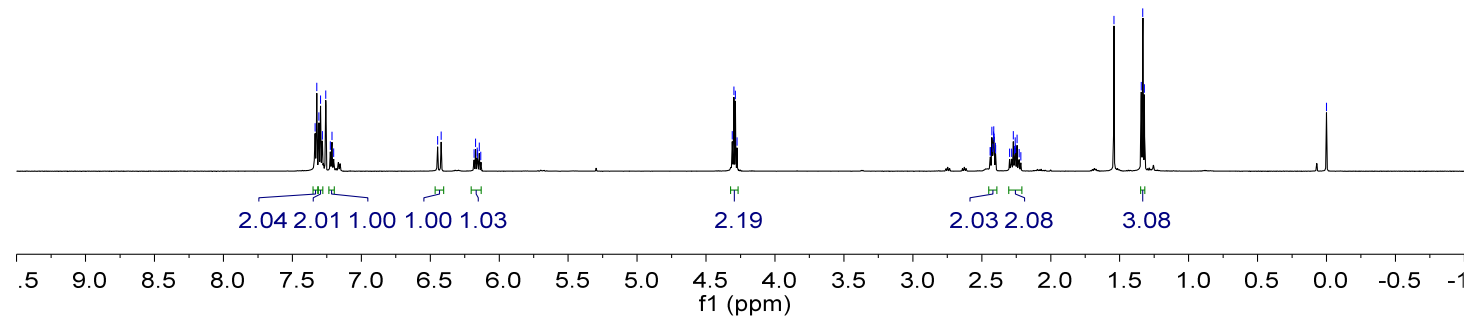



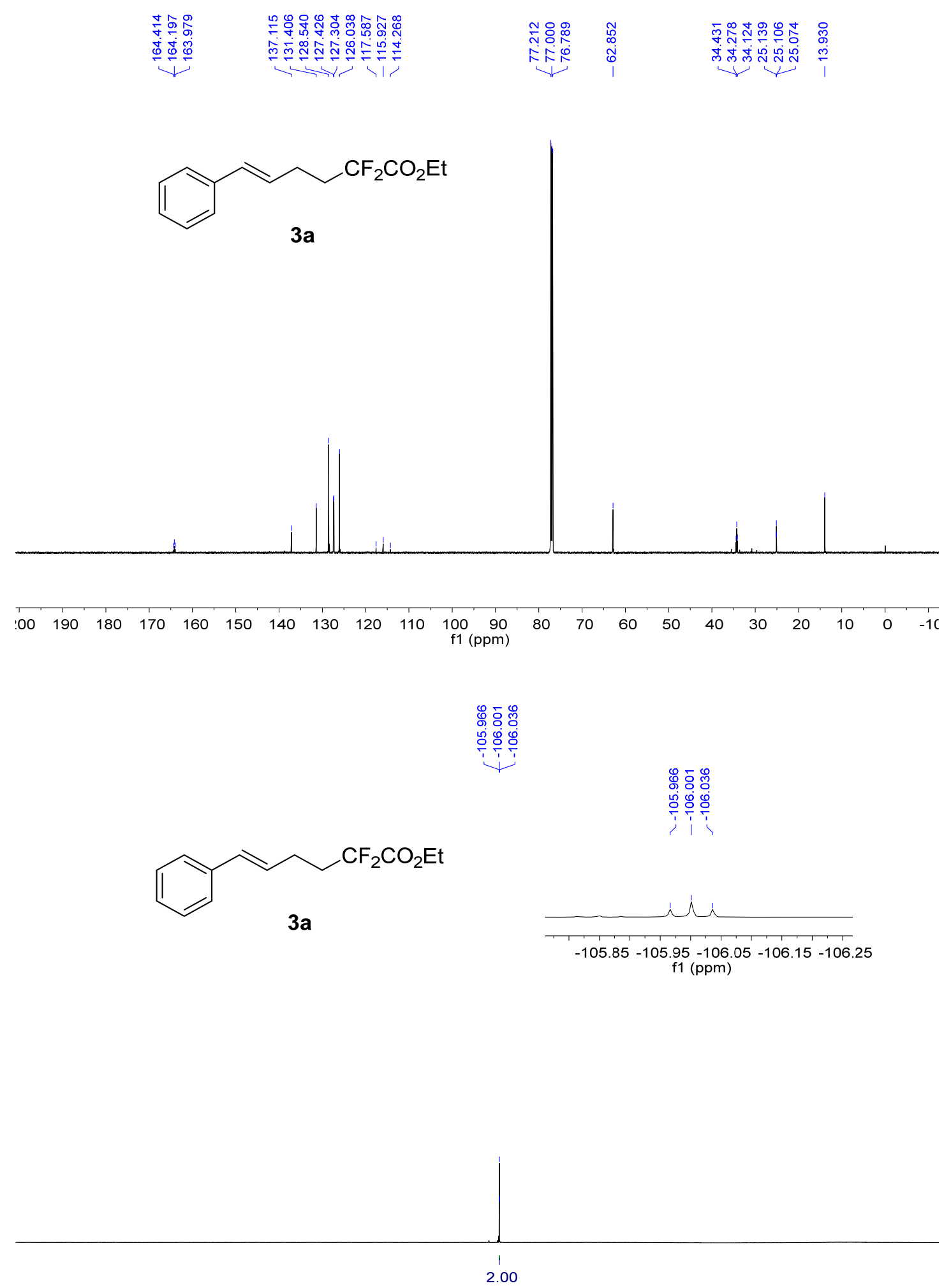

$2010 \quad 0 \quad-10 \quad-20 \quad-30 \quad-40 \quad-50 \quad-60 \quad-70 \quad-80 \quad-90-100-110-120-130-140-150-160-170-180-190-200-210-220$ 
<smiles>CCOC(=O)C(F)(F)CC/C=C/c1ccc(C)cc1</smiles>

$3 \mathbf{b}$

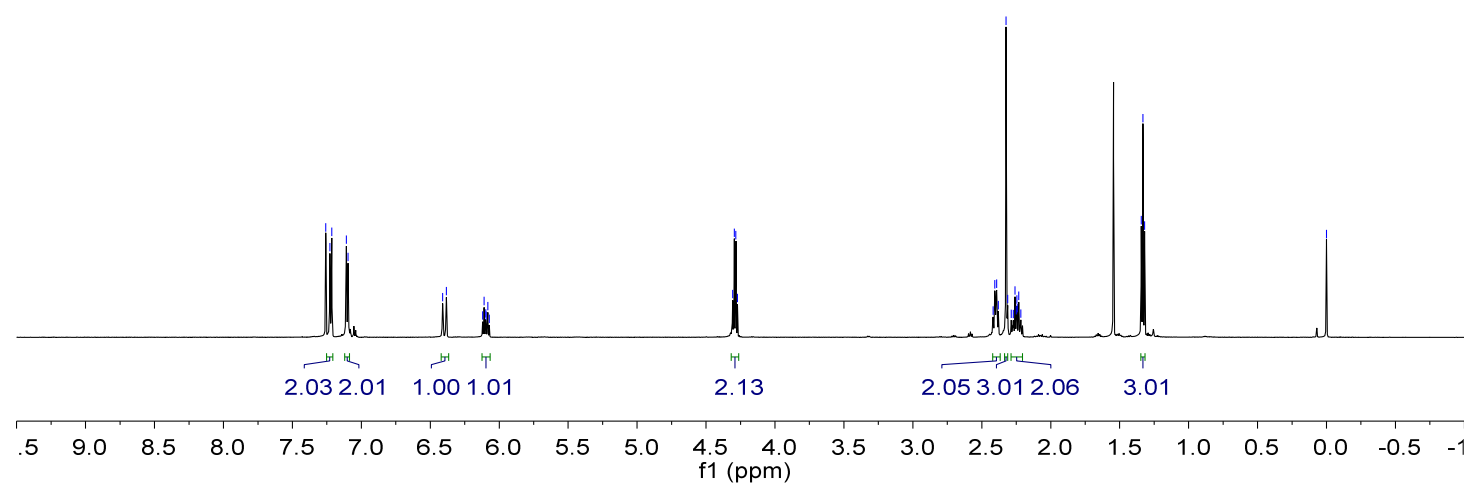

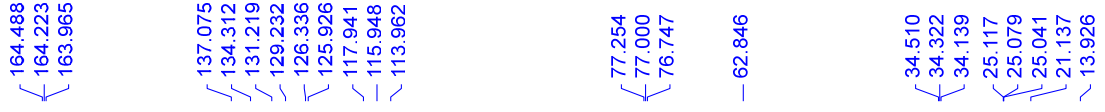

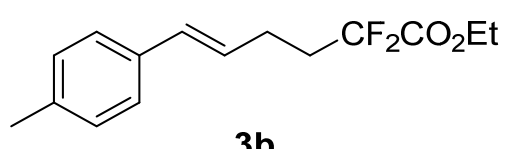

3b

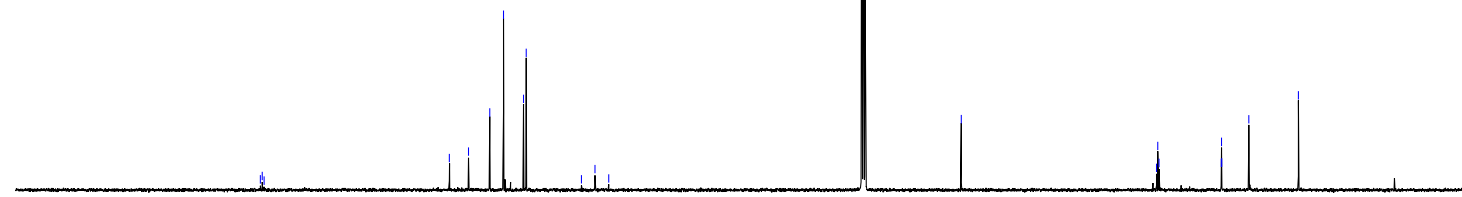
\begin{tabular}{llllllllllllllllllll}
\hline DO & 190 & 180 & 170 & 160 & 150 & 140 & 130 & 120 & 110 & $\begin{array}{c}100 \\
\mathrm{f} 1(\mathrm{ppm})\end{array}$ & 80 & 70 & 60 & 50 & 40 & 30 & 20 & 10 & 0
\end{tabular} 


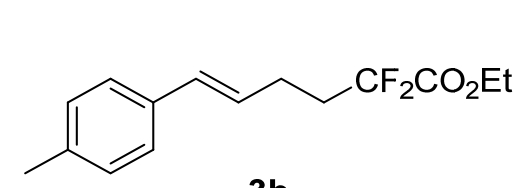

$3 \mathbf{b}$
影赠

융융융

\section{i i}

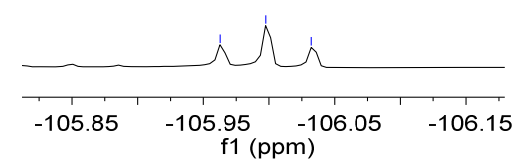

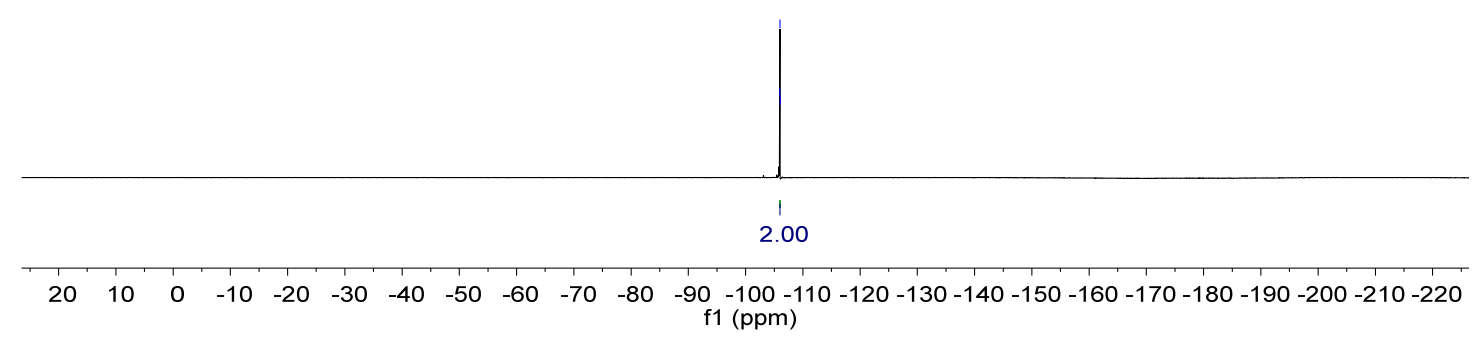

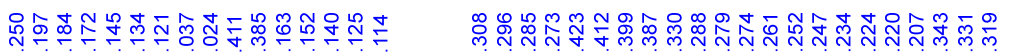

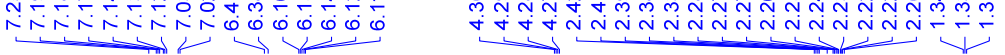<smiles>CCOC(=O)CCC/C=C/c1cccc(C)c1</smiles>

$3 c$

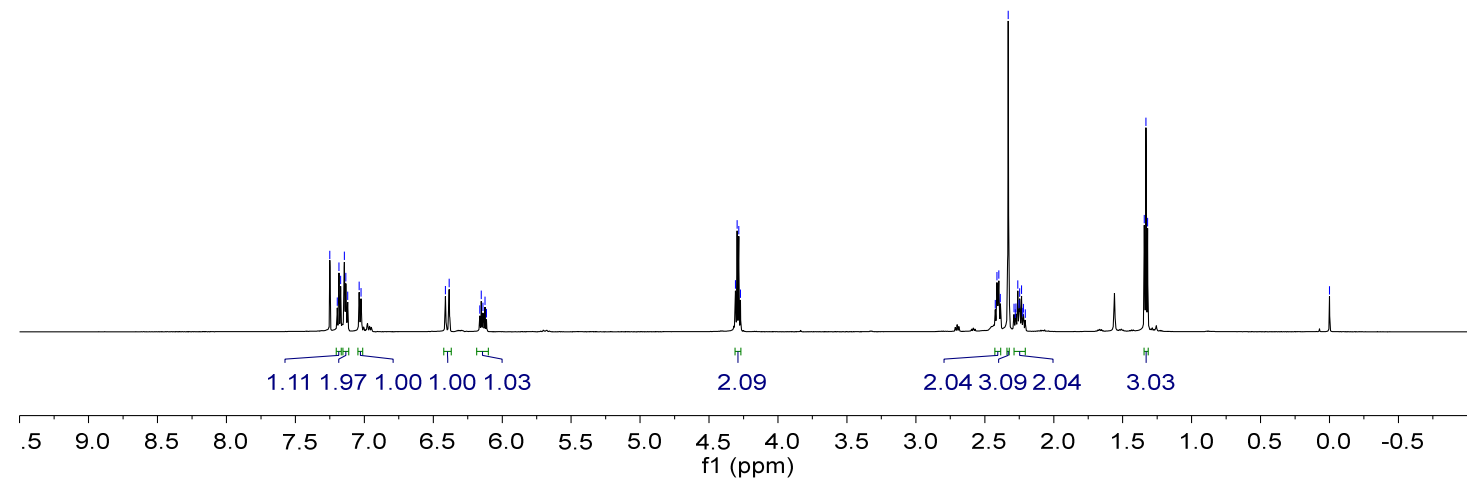




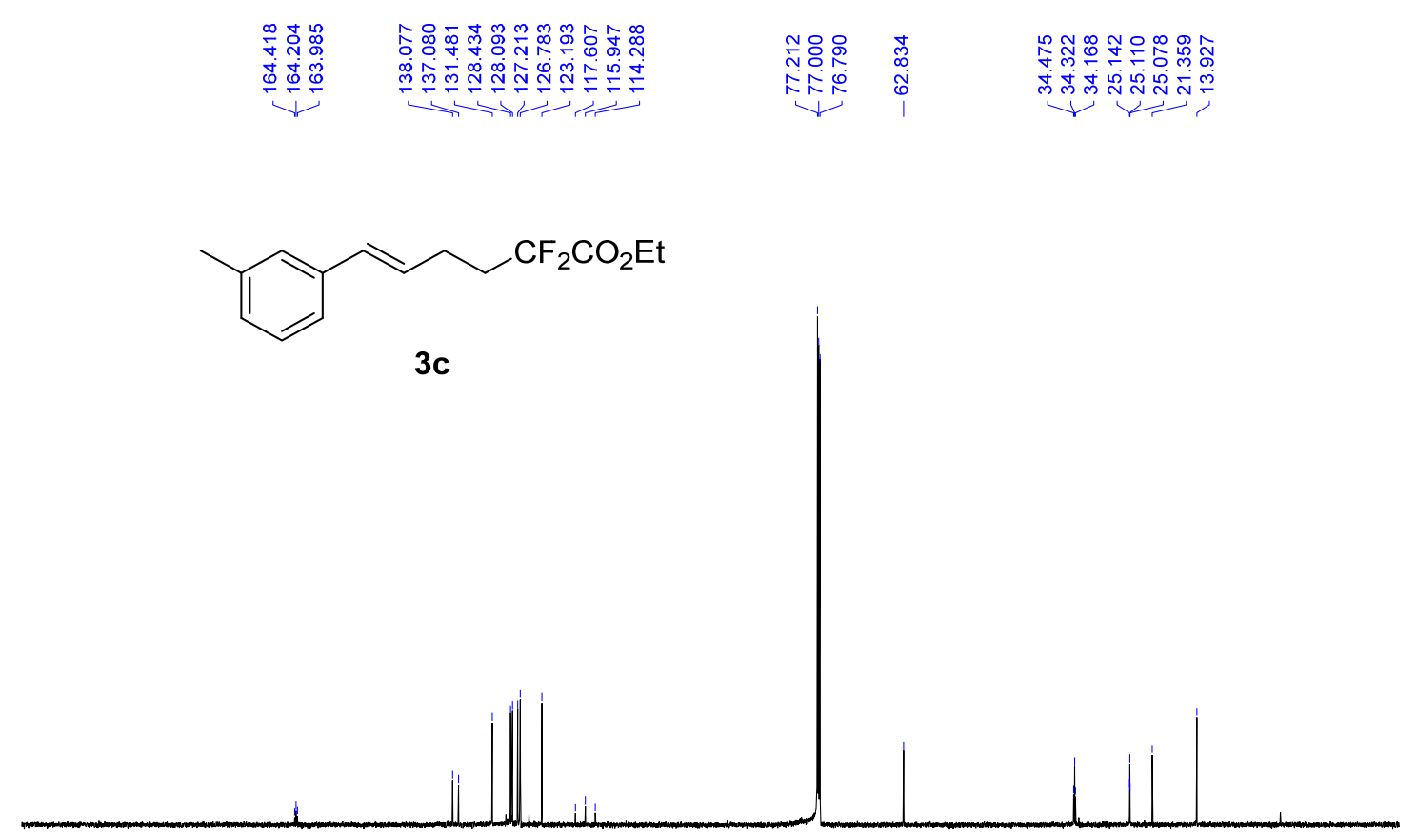

\begin{tabular}{lllllllllllllllllllllllllll}
\hline 10 & 200 & 190 & 180 & 170 & 160 & 150 & 140 & 130 & 120 & 110 & $\begin{array}{l}100 \\
\mathrm{f} 1(\mathrm{ppm})\end{array}$ & 80 & 70 & 60 & 50 & 40 & 30 & 20 & 10 & 0 & -10 & -4
\end{tabular}

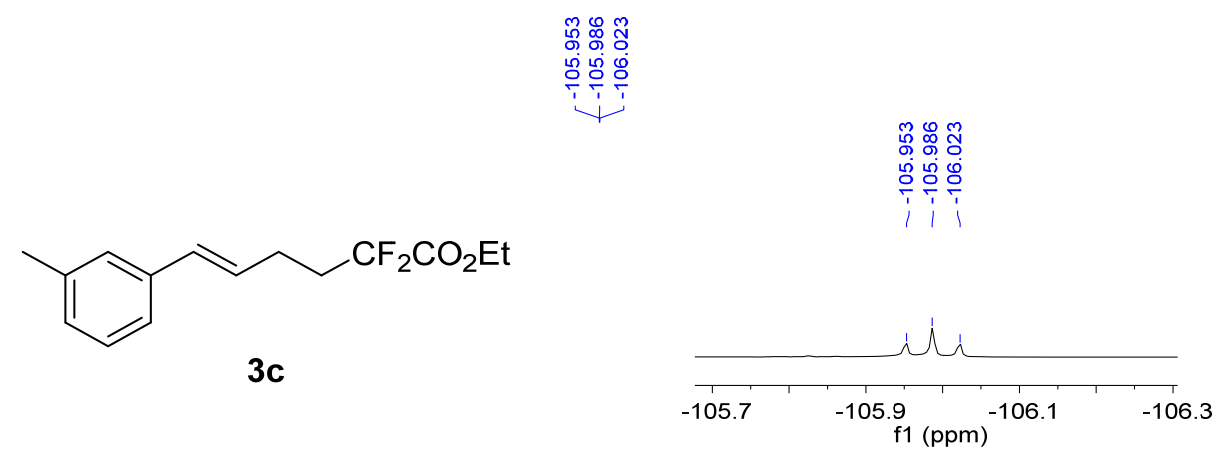

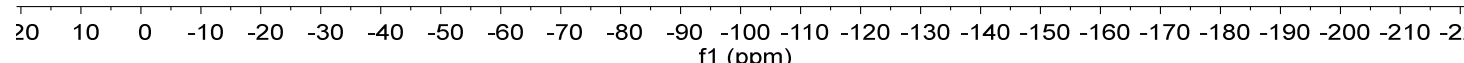




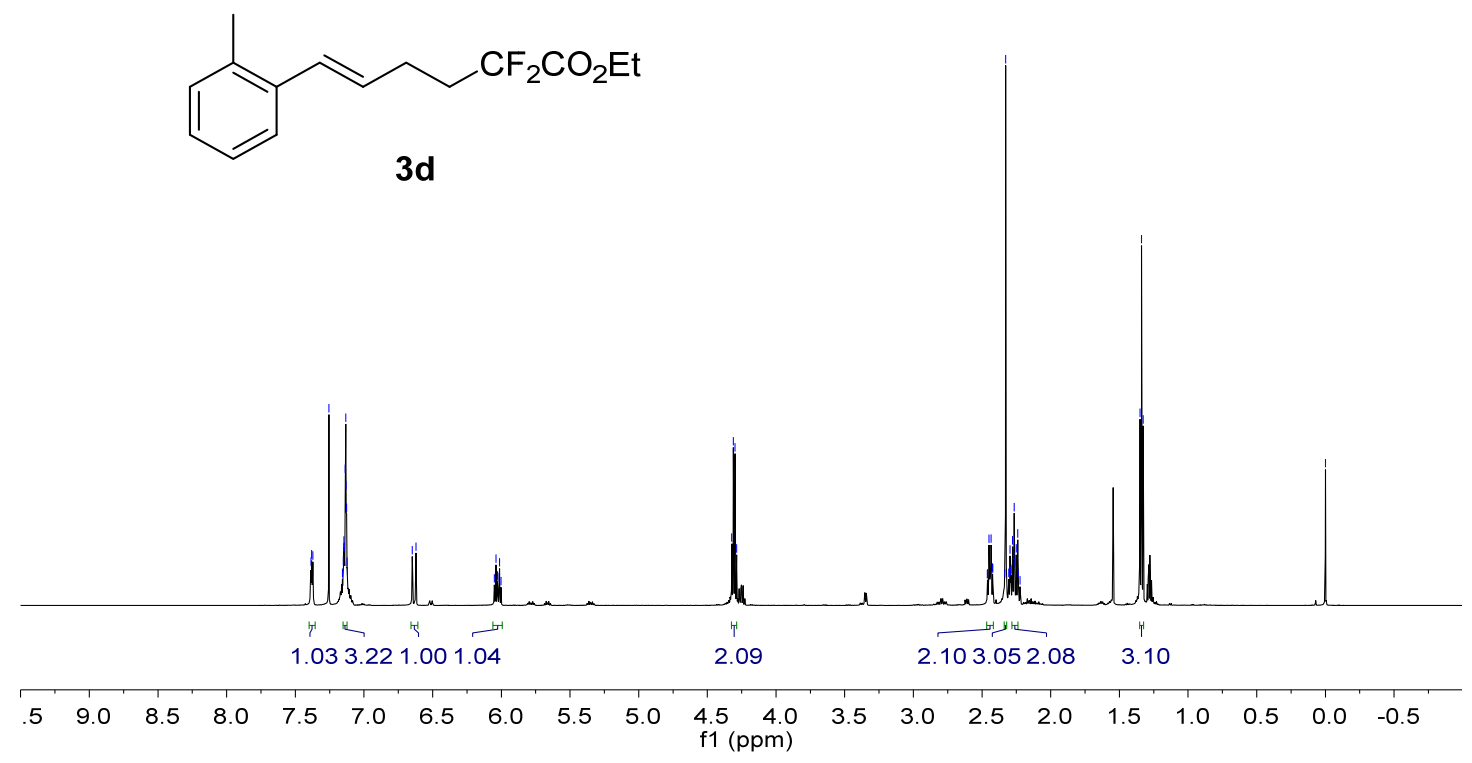

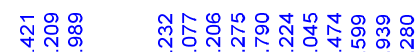

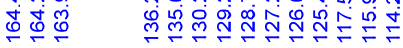

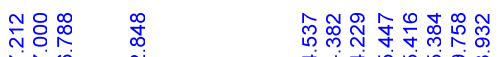

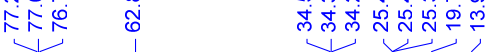

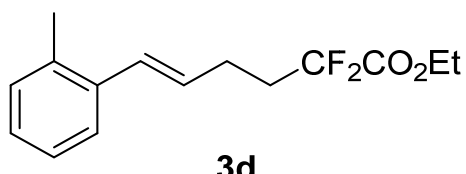

$3 d$

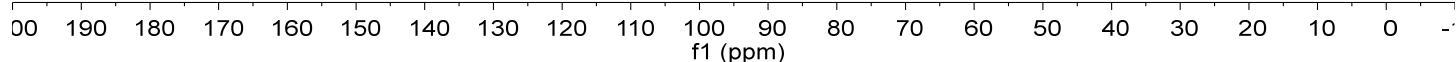



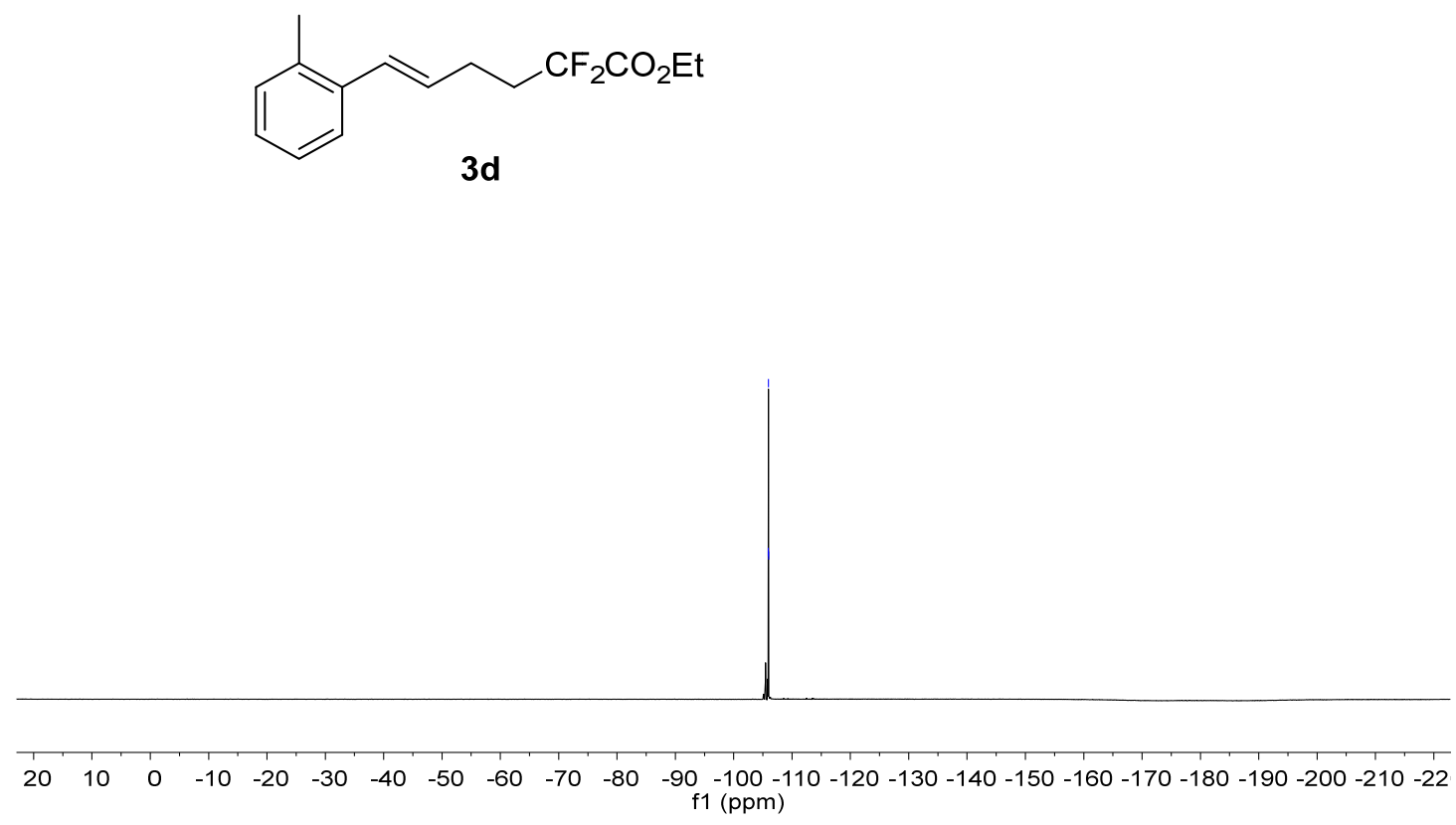

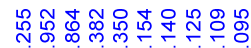

N

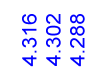

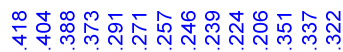

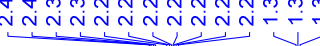

웅<smiles>CCOC(=O)CCC/C=C/c1cc(C)cc(C)c1</smiles>

$3 e$

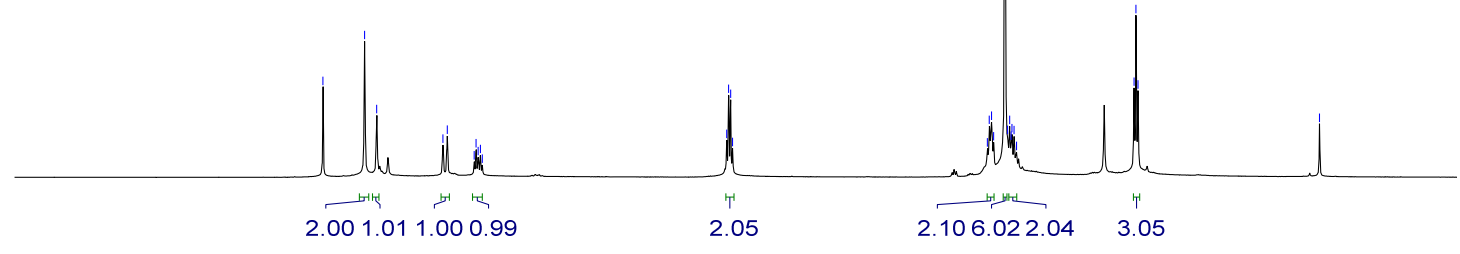

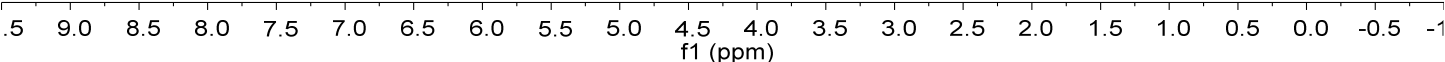



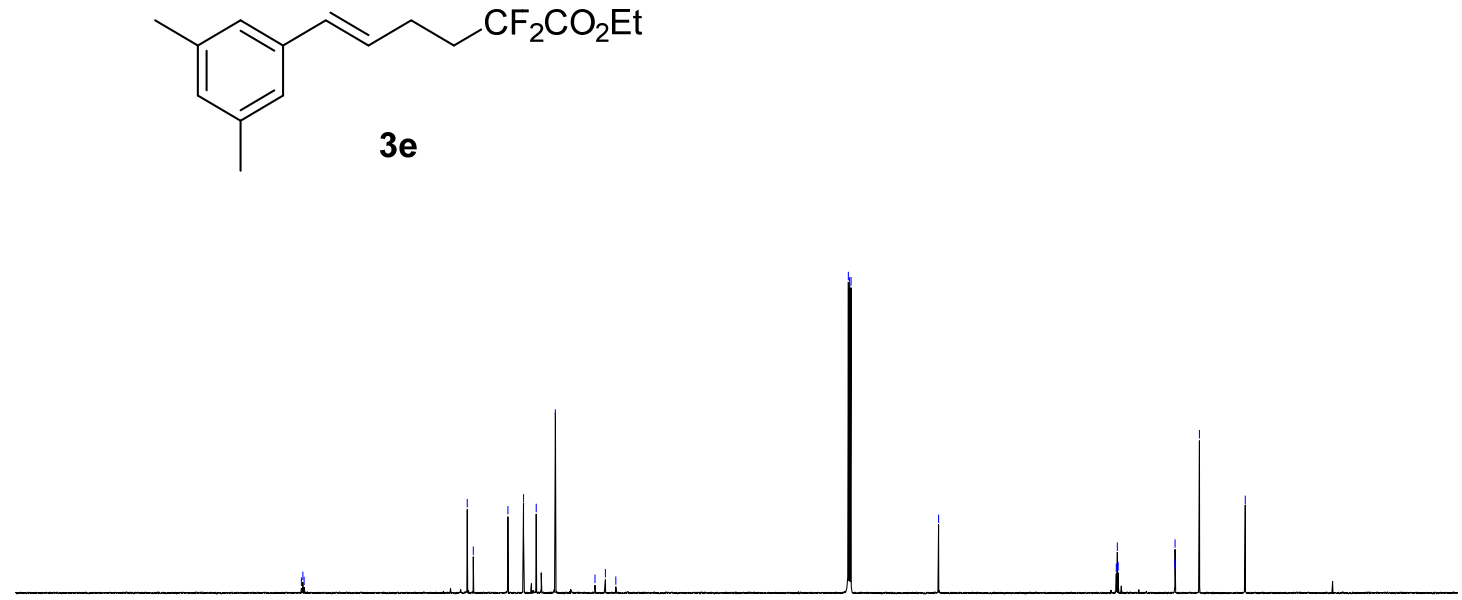

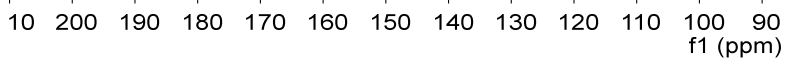

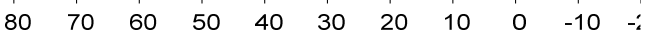

ธॅㅎํ용

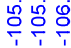

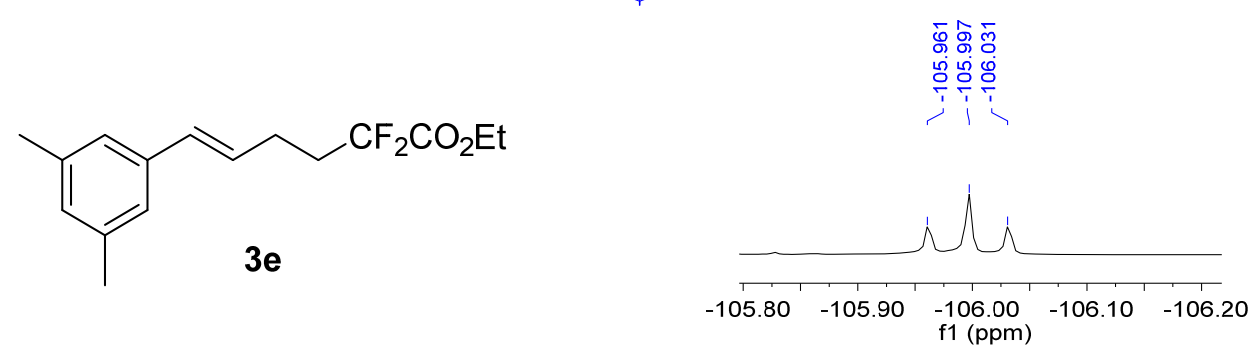

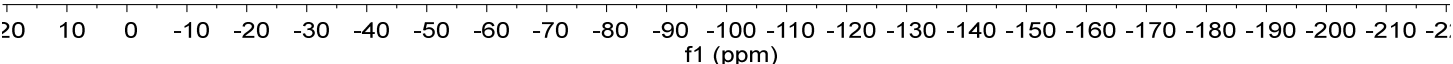


<smiles>CCOC(=O)CCC/C=C/c1ccc(CC)cc1</smiles>

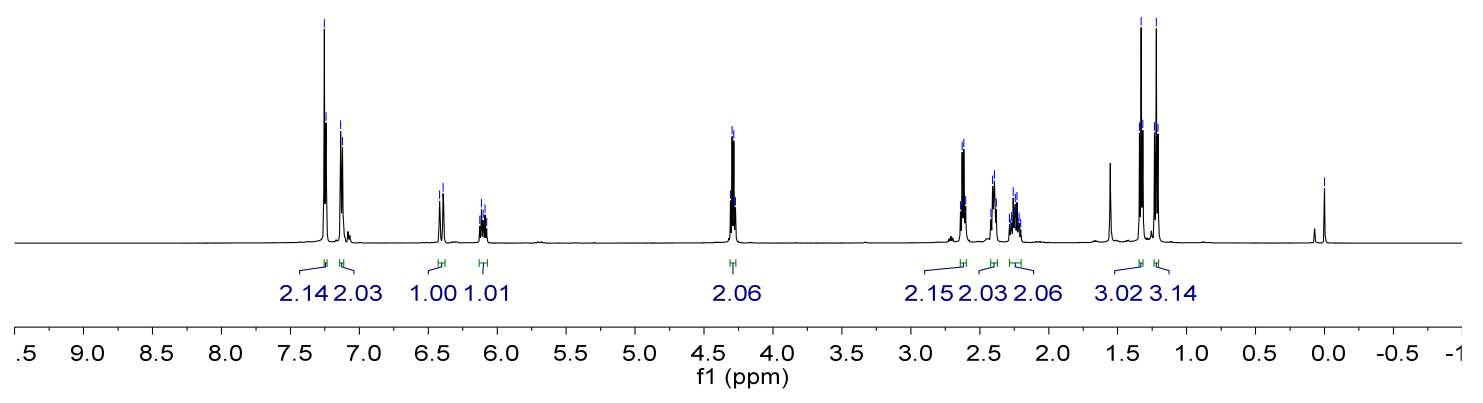

$\underbrace{\text { l }}$
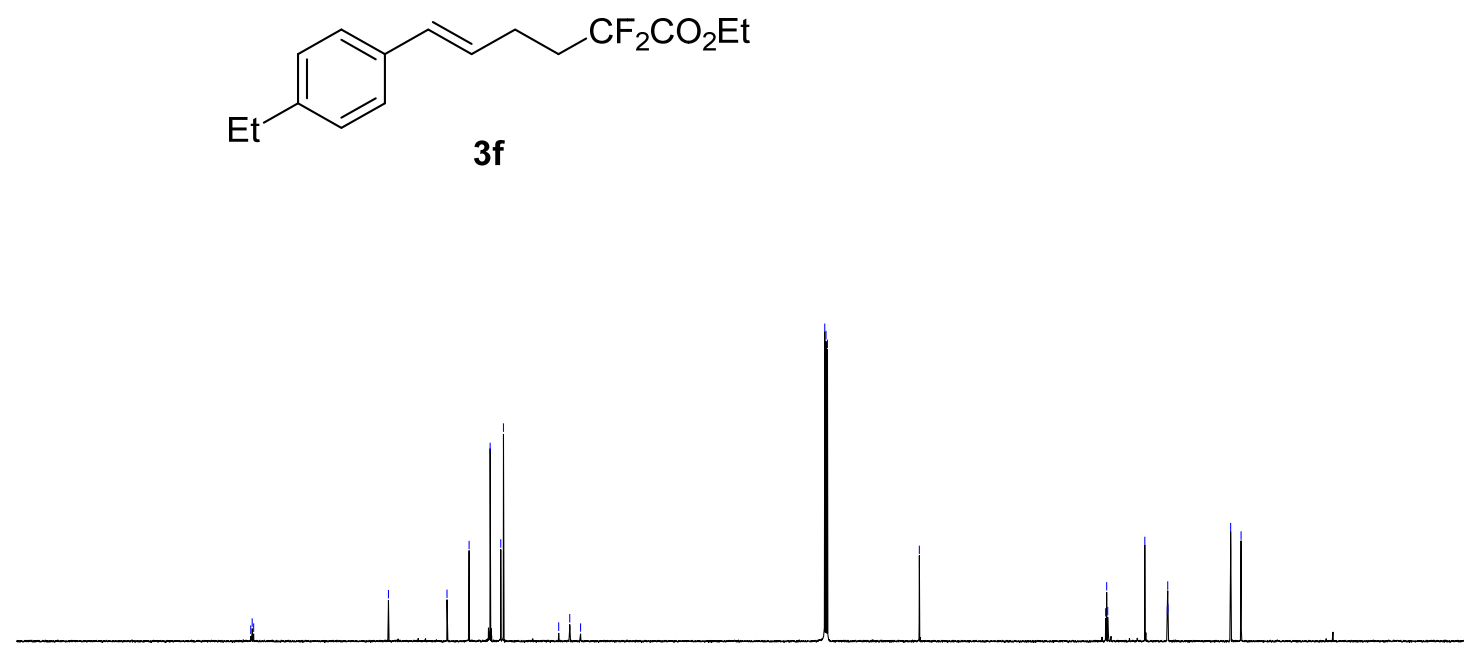

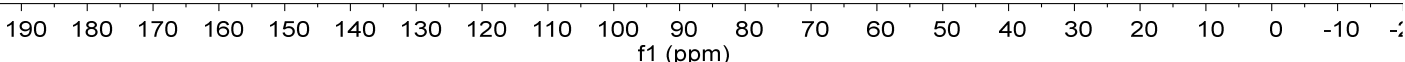



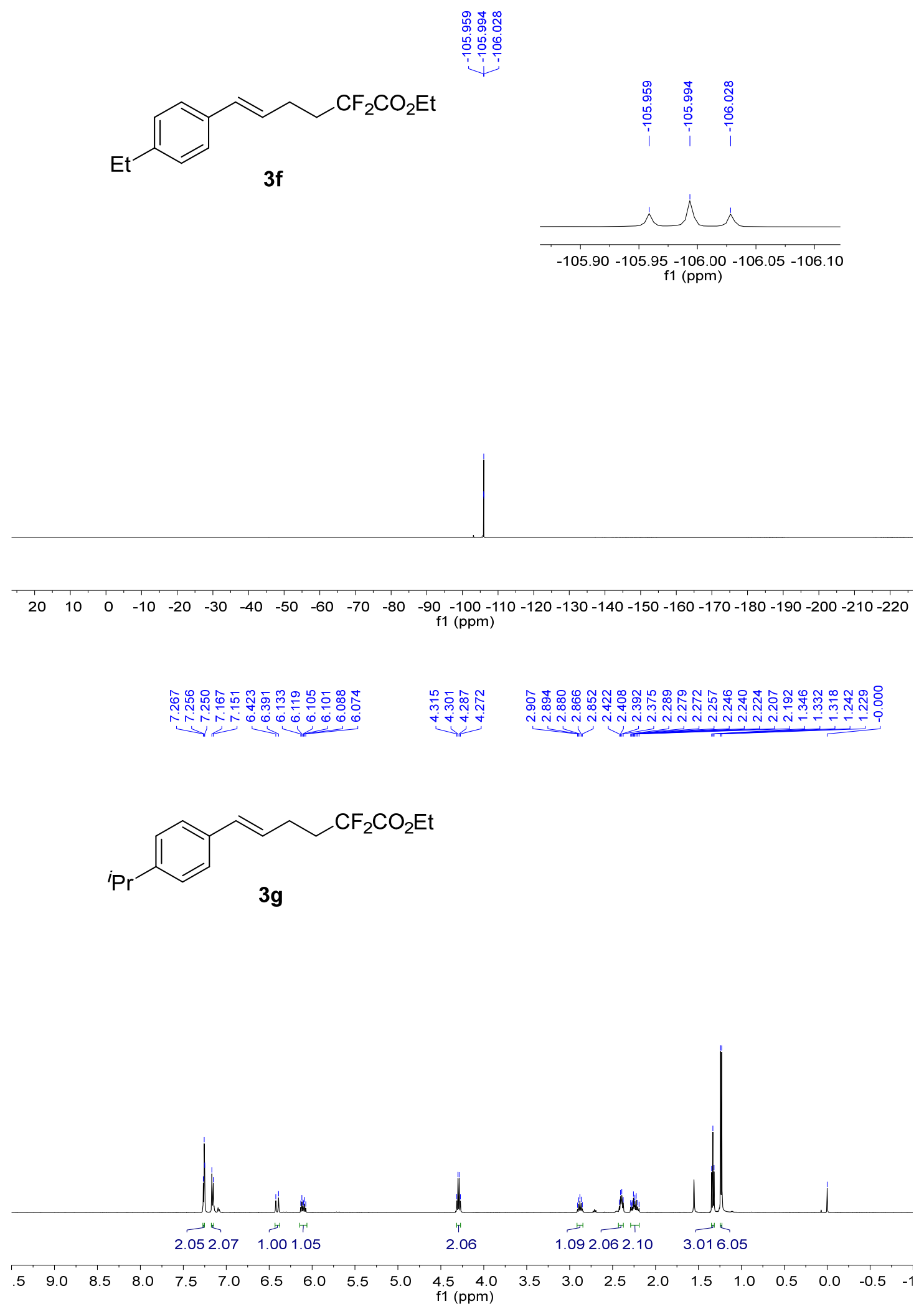


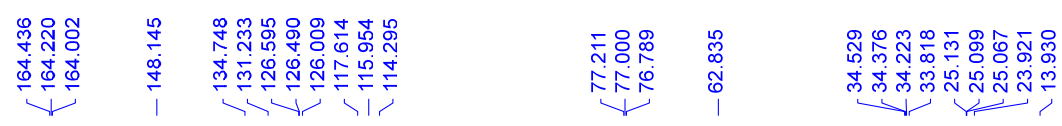

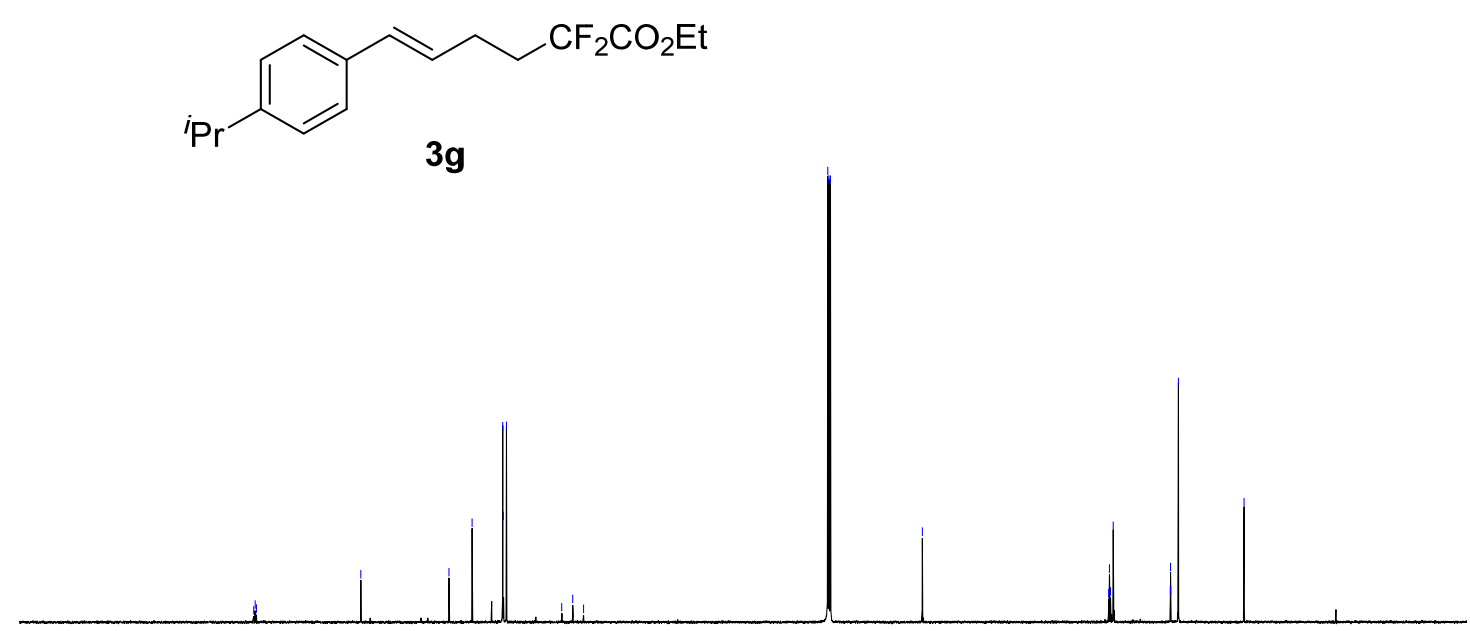

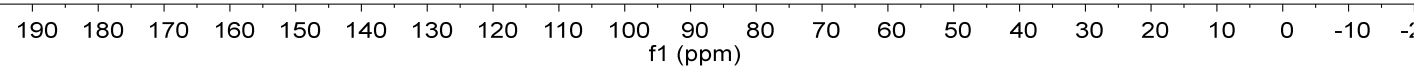

\section{ఊั}

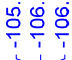

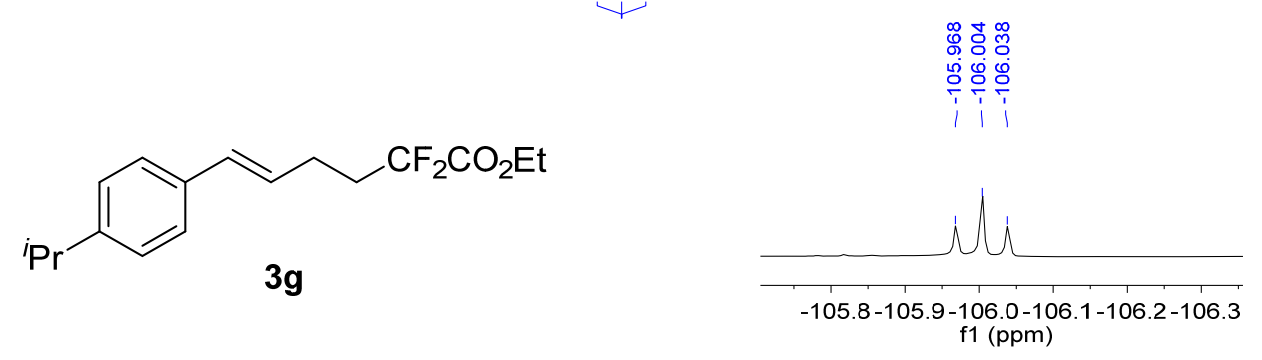

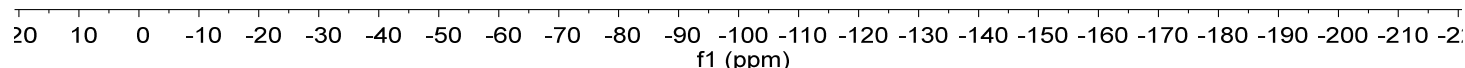




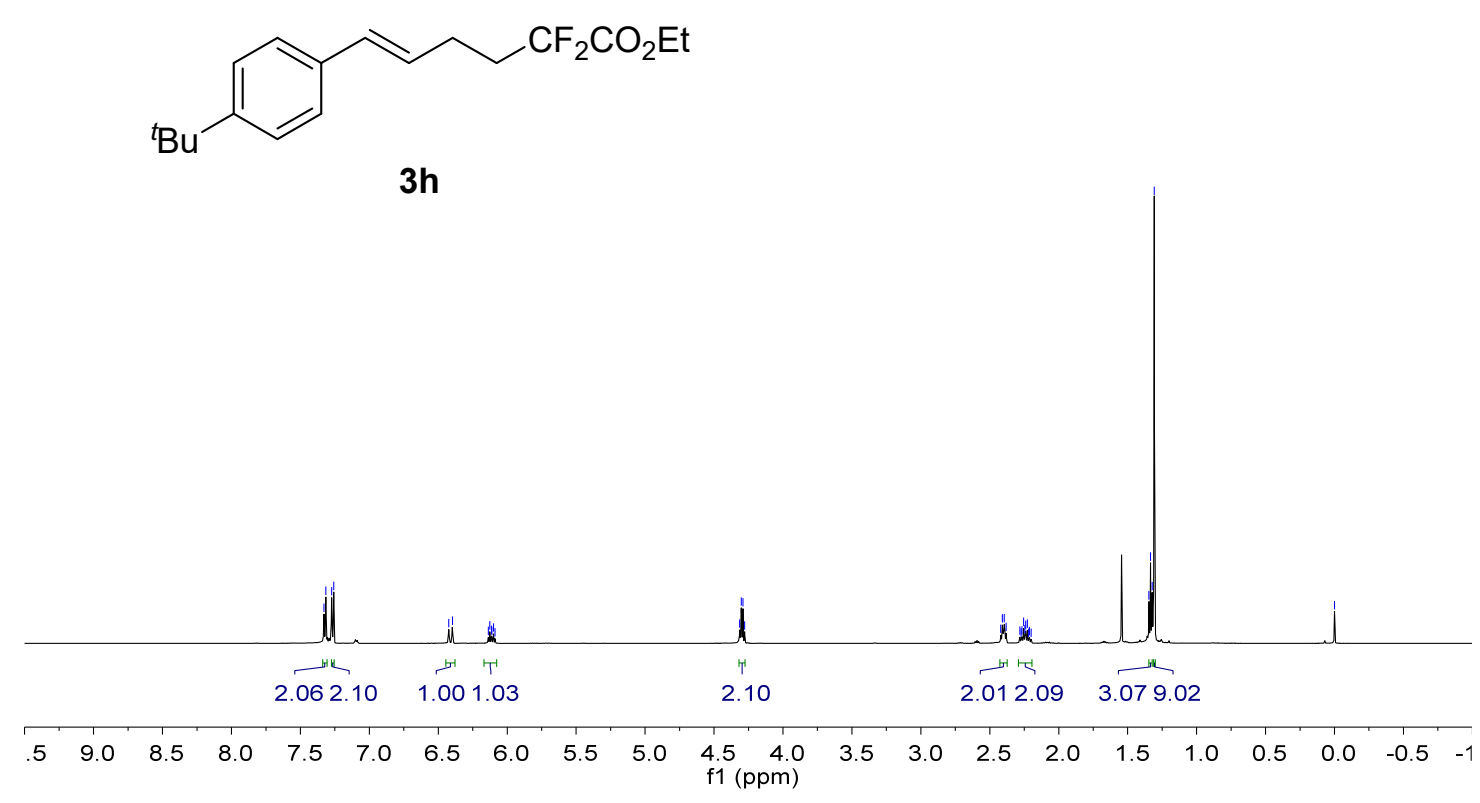

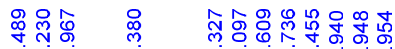

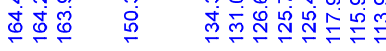

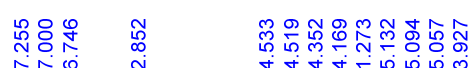

岤

牙它,

रNं

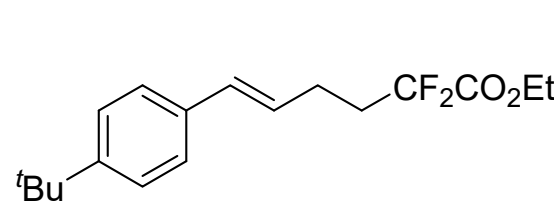

$3 h$

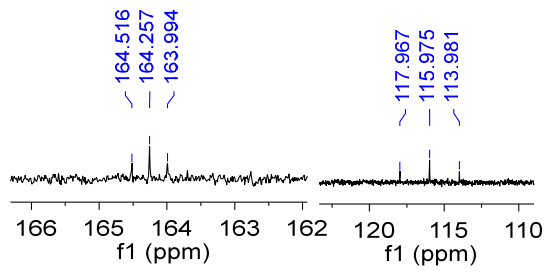

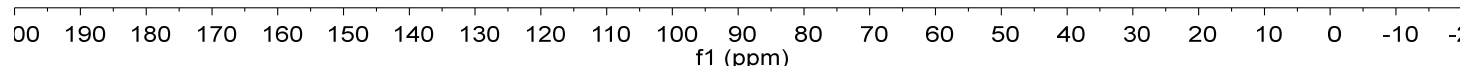



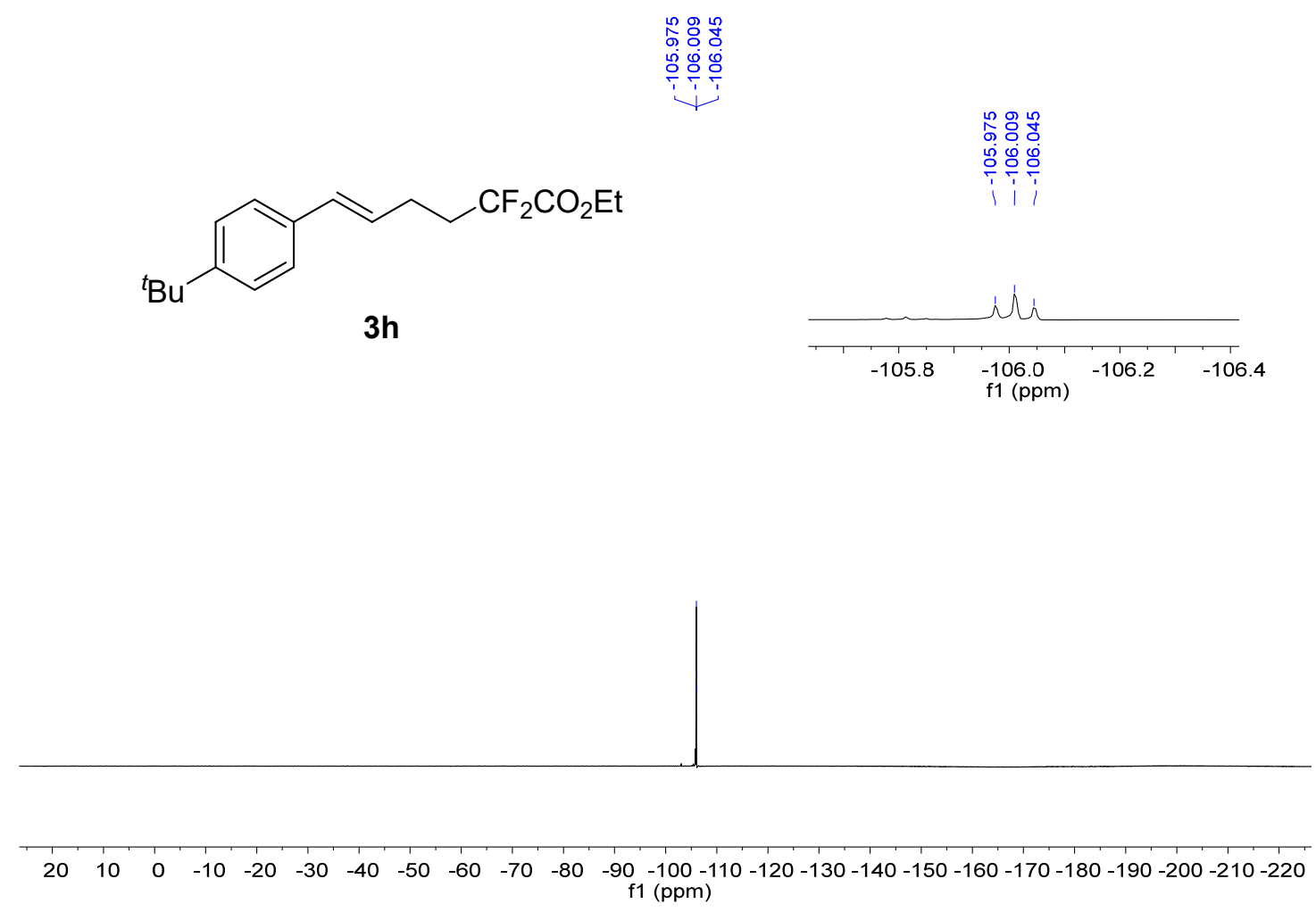

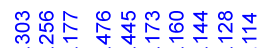

分分

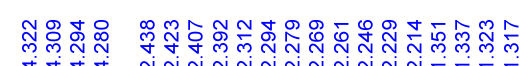

8
0
0
1<smiles>CCOC(=O)C(F)(F)CCC/C=C/c1cc(C(C)(C)C)cc(C(C)(C)C)c1</smiles>

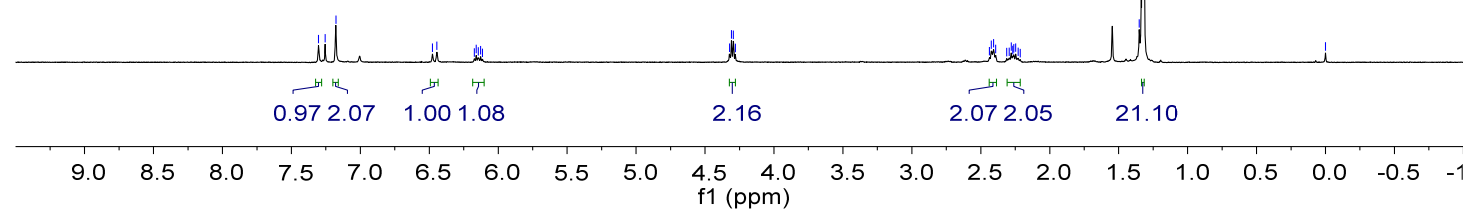




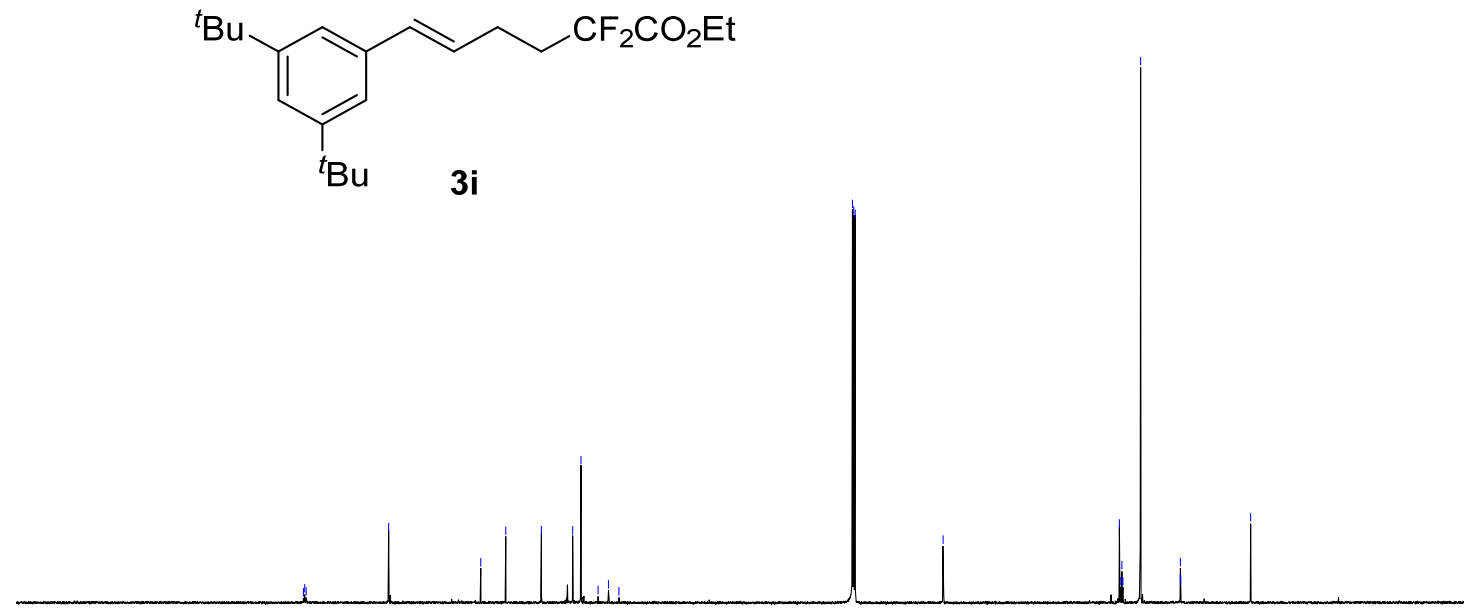

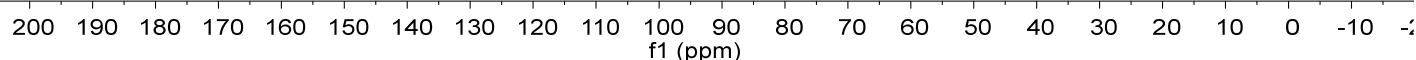

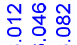

它审

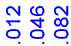

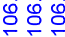

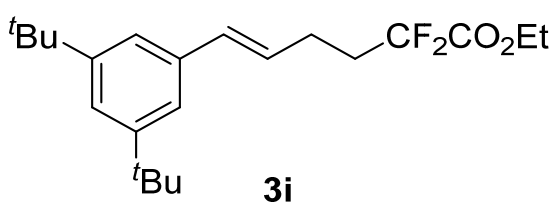

$i \frac{1}{i}$

${ }^{t} \mathrm{Bu} \quad 3 \mathbf{i}$

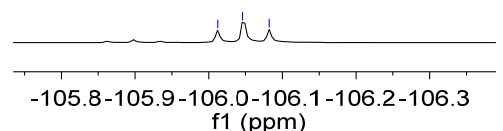

$\mathrm{f1}(\mathrm{ppm})$

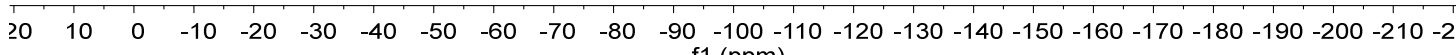
f1 (ppm) 
<smiles>CCOC(=O)CCC/C=C/c1ccc(-c2ccccc2)cc1</smiles>

3j

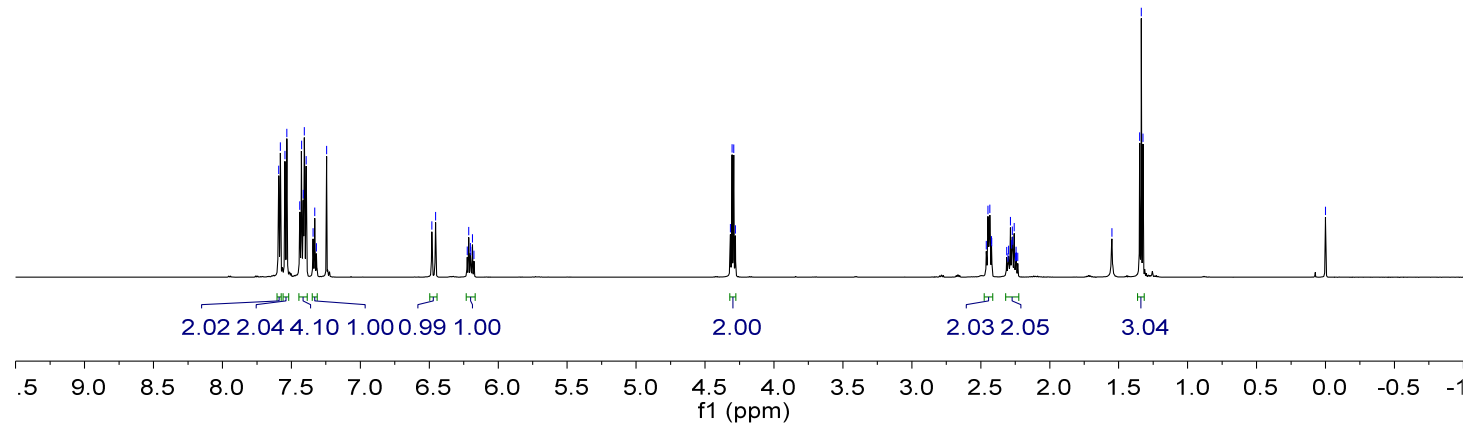

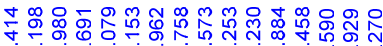

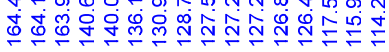

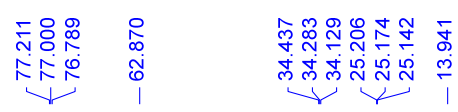<smiles>CCOC(=O)CCC/C=C/c1ccc(-c2ccccc2)cc1</smiles>

3j

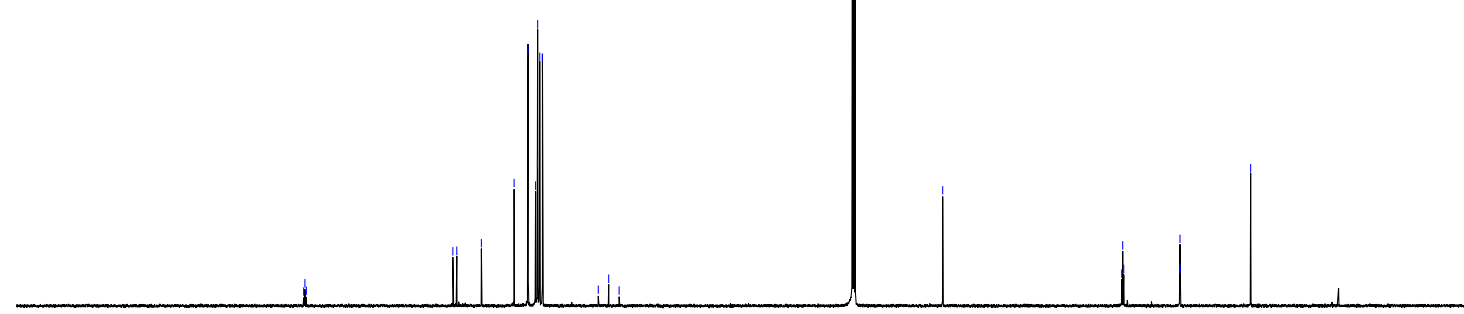

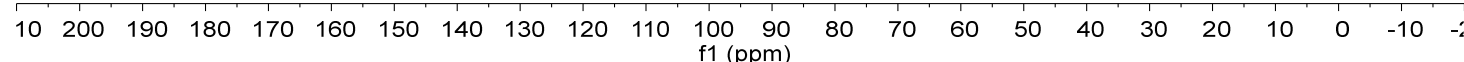




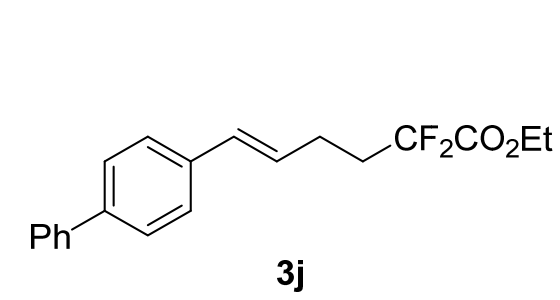

앵중영

웅 윰웅

3j

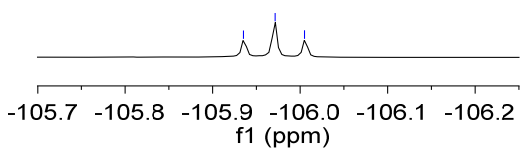

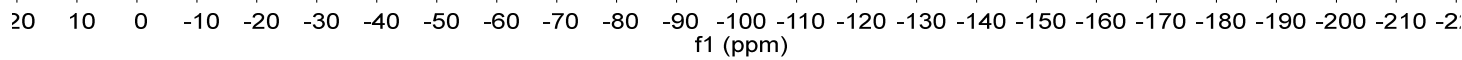

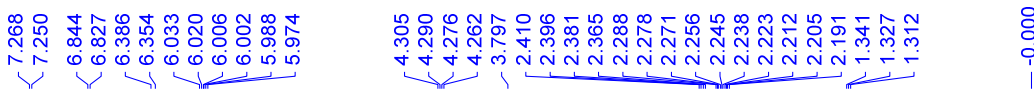

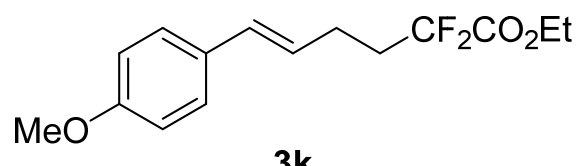

3k

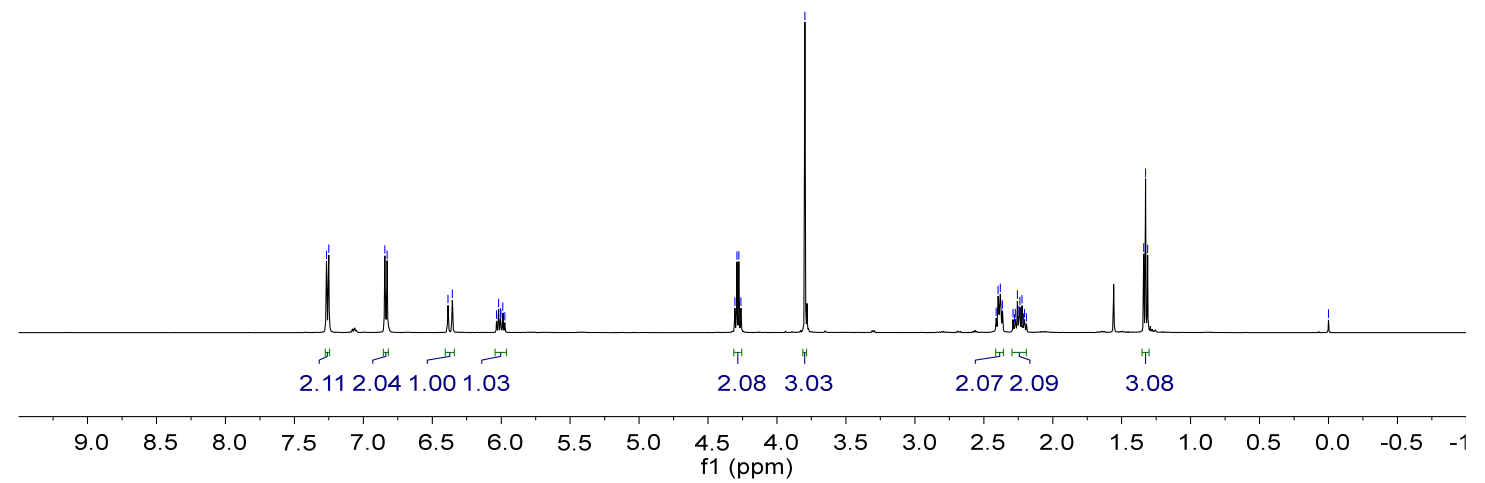




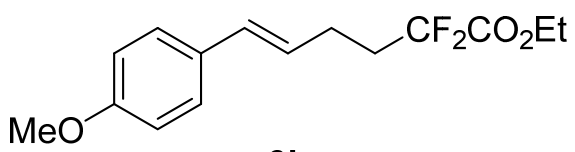

$3 k$

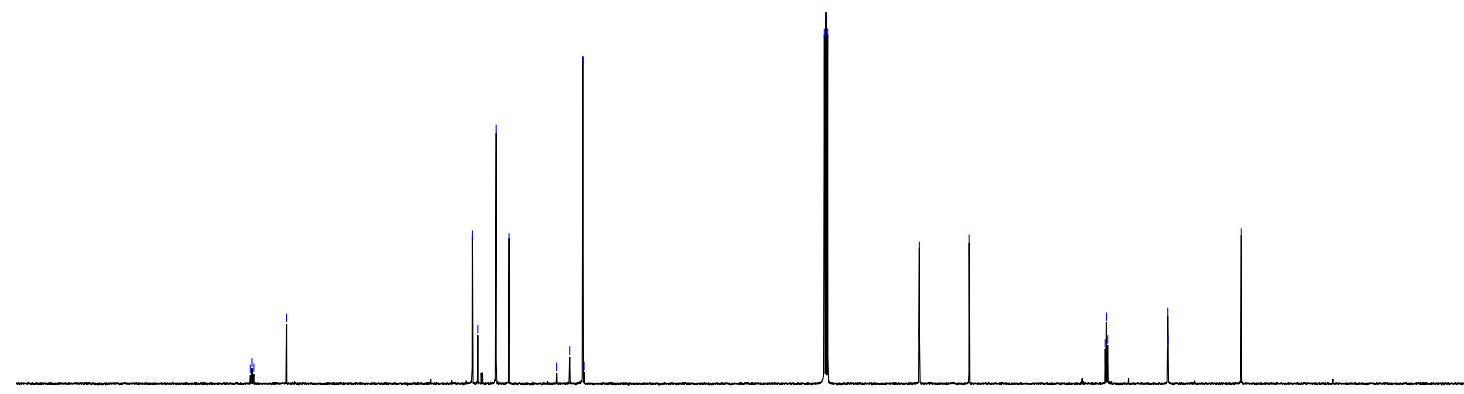

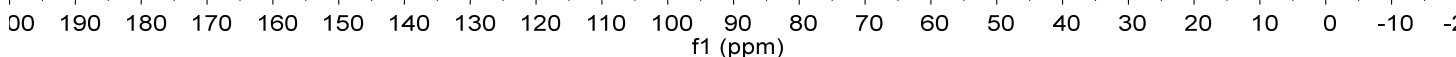

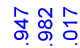

ํํㅇำ<smiles>CCOC(=O)CCCC=Cc1ccc(OC)cc1</smiles>

$3 k$

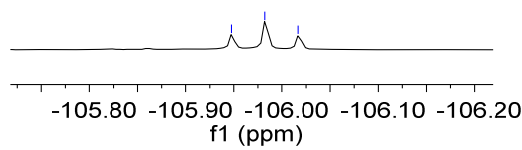

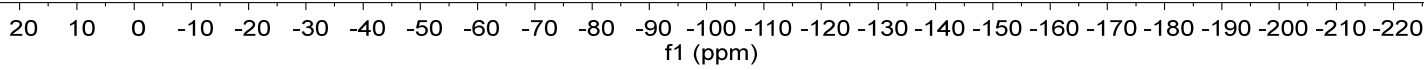



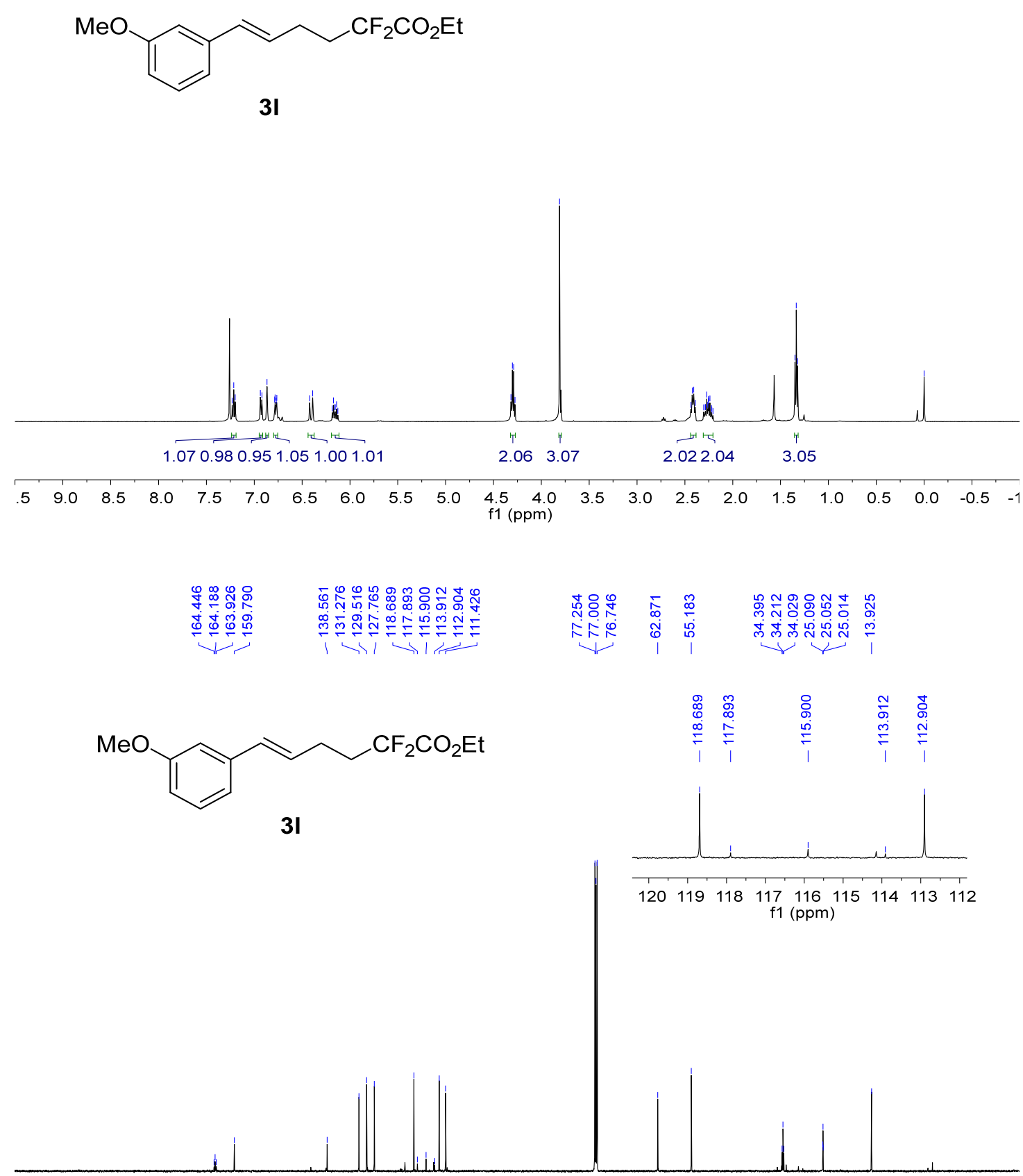

$\begin{array}{llllllllllllllllllllllllll}10 & 200 & 190 & 180 & 170 & 160 & 150 & 140 & 130 & 120 & 110 & \begin{array}{r}100 \\ \mathrm{f} 1(\mathrm{ppm})\end{array} & 80 & 70 & 60 & 50 & 40 & 30 & 20 & 10 & 0 & -10 & -:\end{array}$ 

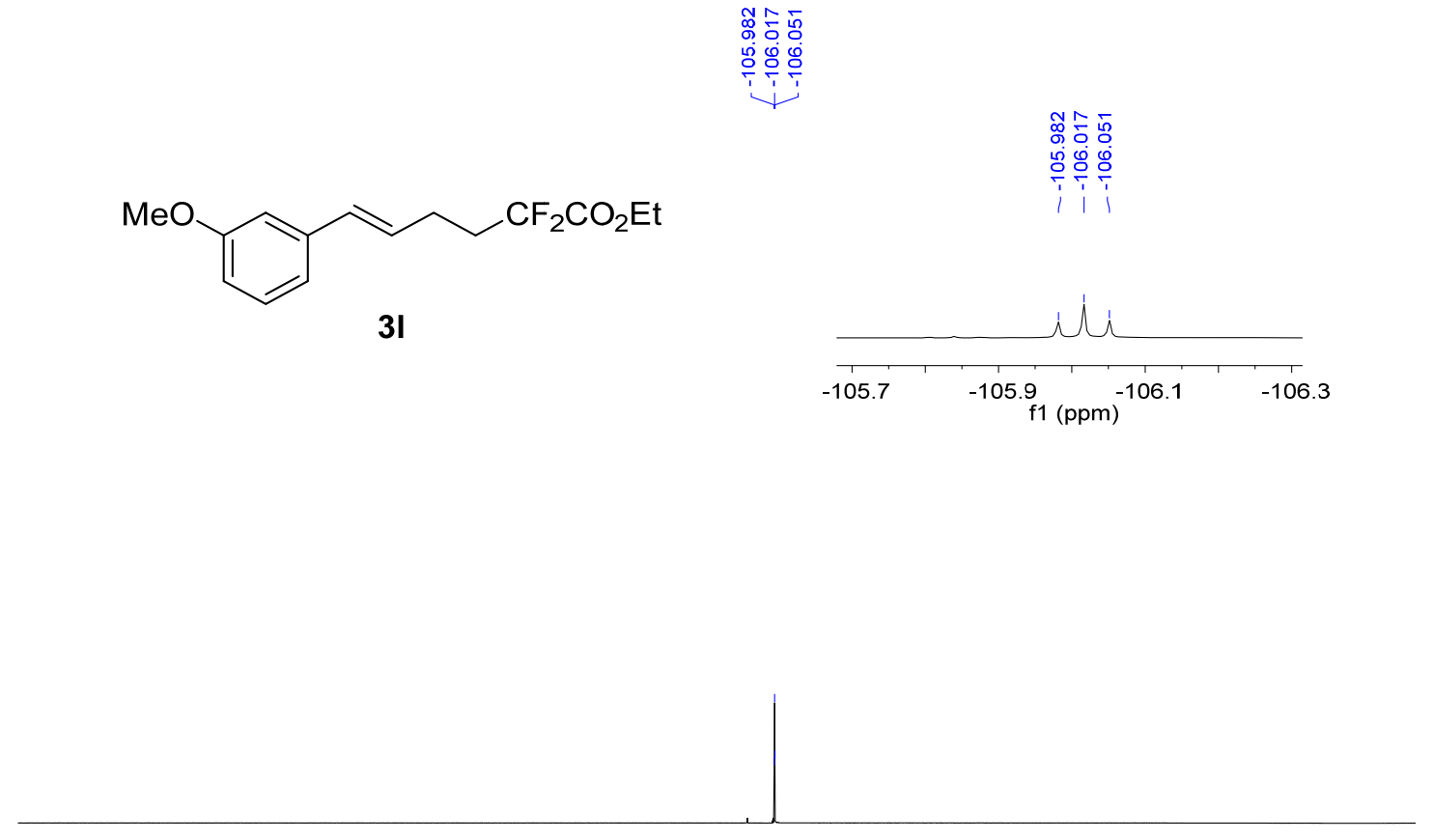

\begin{tabular}{|c|c|c|c|c|c|c|c|c|c|c|c|c|c|c|}
\hline & -40 & -50 & -60 & -70 & -80 & -90 & $\begin{array}{c}-100 \\
\mathrm{f} 1 \text { (ppm) }\end{array}$ & -110 & -120 & -130 & -140 & -150 & -160 & -170 \\
\hline & & & $\begin{array}{ll}0 \\
5\end{array}$ & $\underset{\sim}{\mathcal{Z}} \stackrel{\infty}{\sim} \stackrel{\sim}{\sim}$ & 它 & & 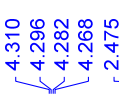 & 官 & רָ & శ్లెల & : & 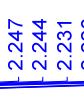 & 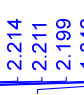 & 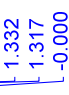 \\
\hline
\end{tabular}

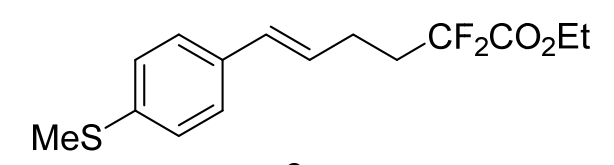

$3 \mathrm{~m}$

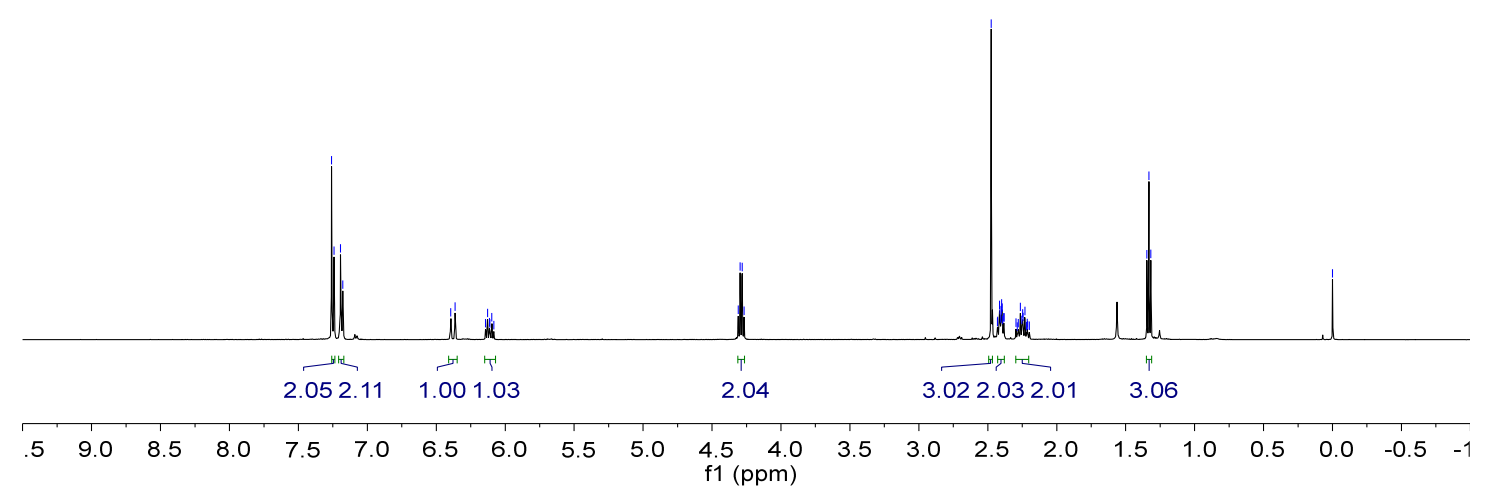




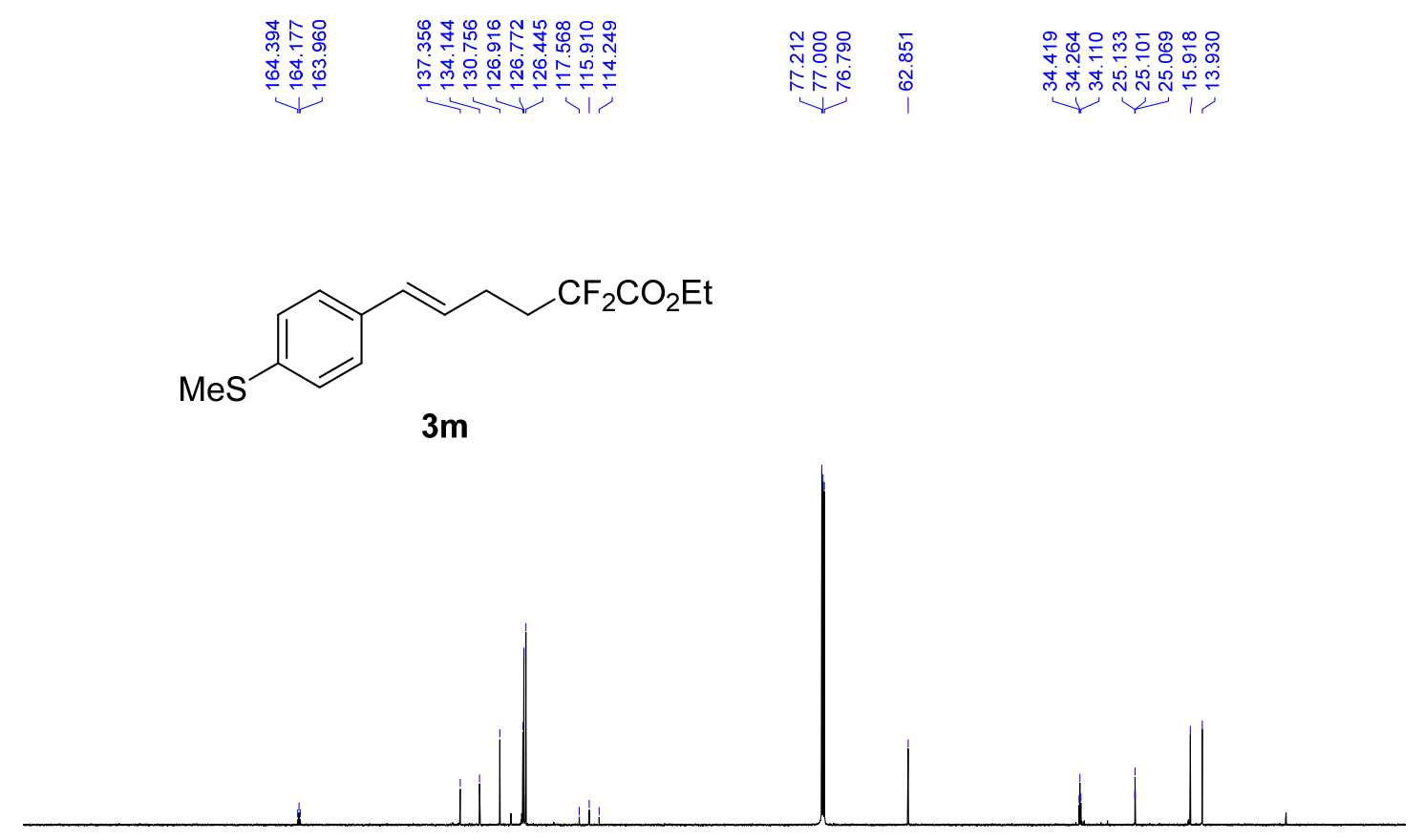

$\begin{array}{llllllllllllllllllllllll}10 & 200 & 190 & 180 & 170 & 160 & 150 & 140 & 130 & 120 & 110 & \begin{array}{r}100 \\ \mathrm{f} 1(\mathrm{ppm})\end{array} & 80 & 70 & 60 & 50 & 40 & 30 & 20 & 10 & 0 & -10 & -4\end{array}$

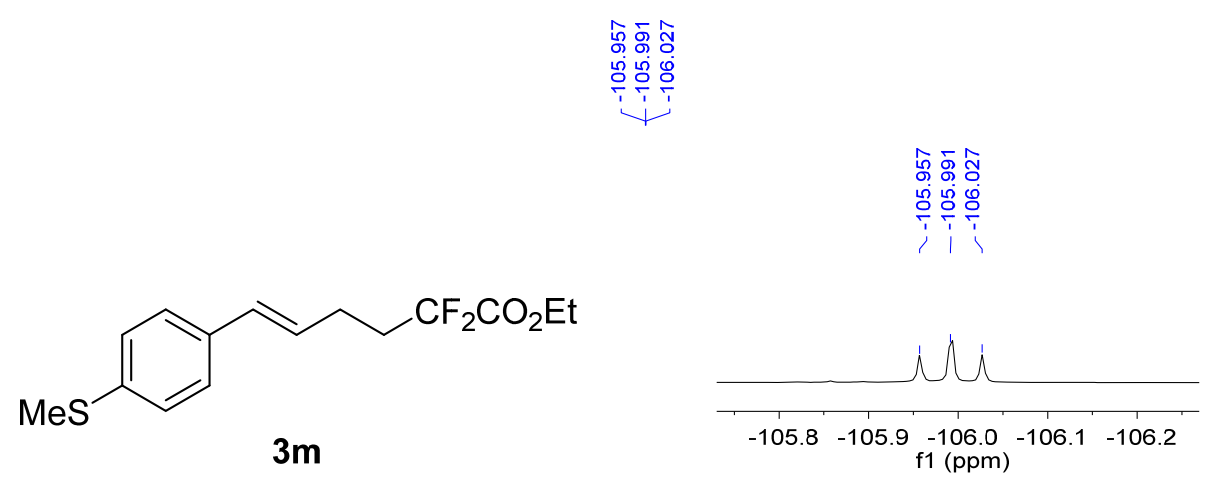

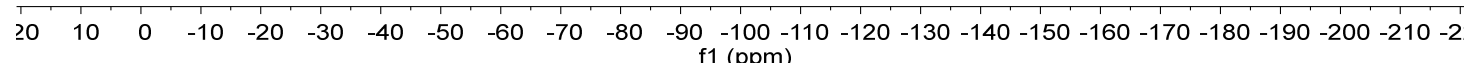



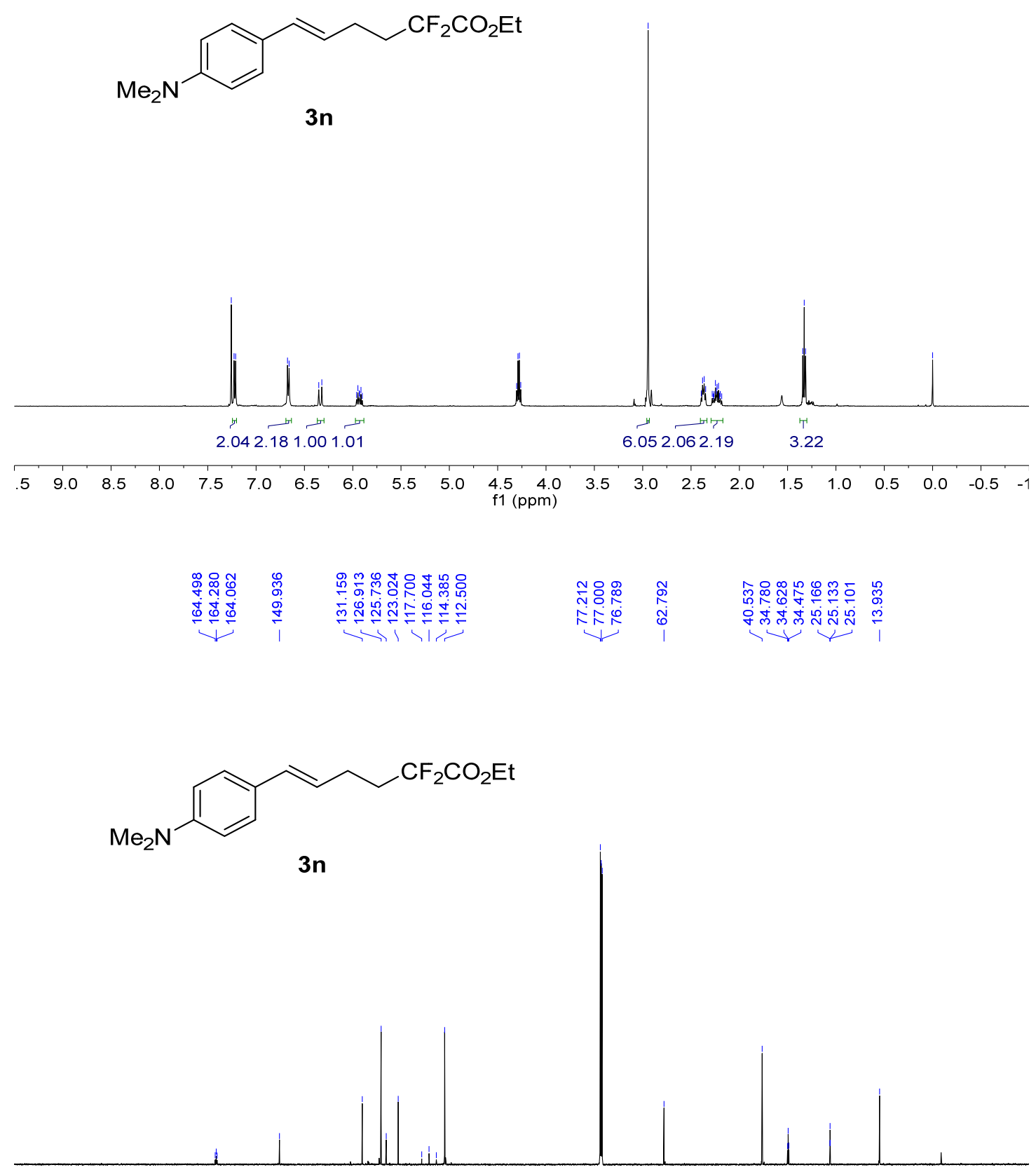

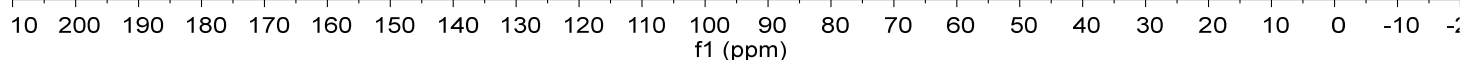




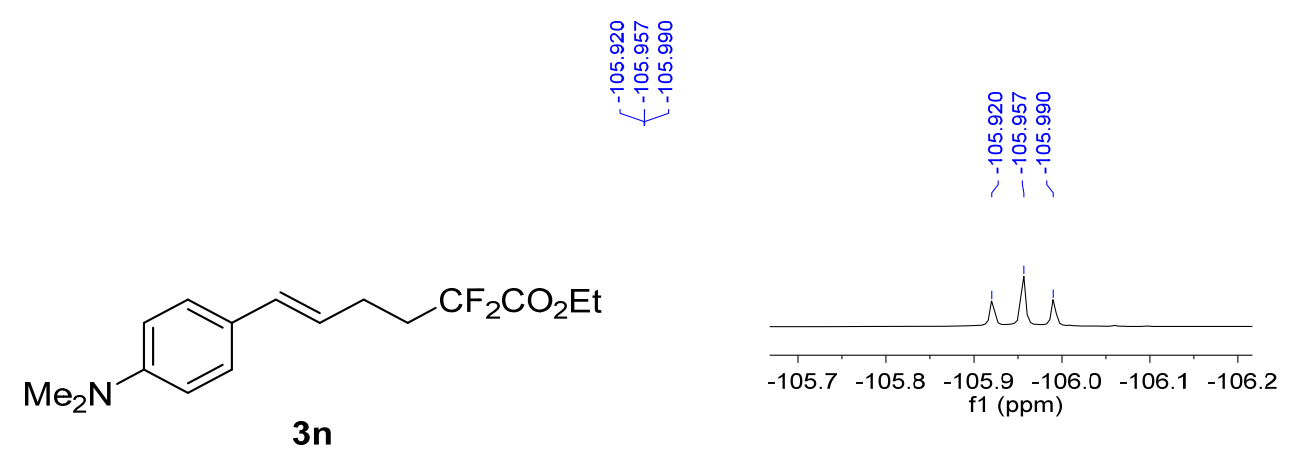

$3 n$

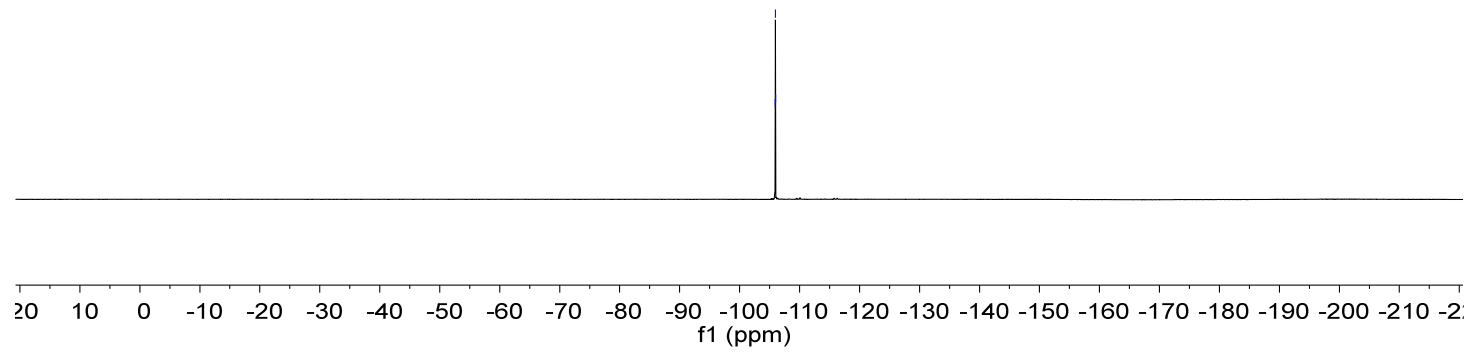

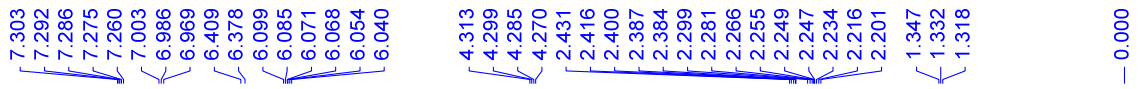<smiles>CCOC(=O)[C-](CC)CCC=Cc1ccc(F)cc1</smiles>

30

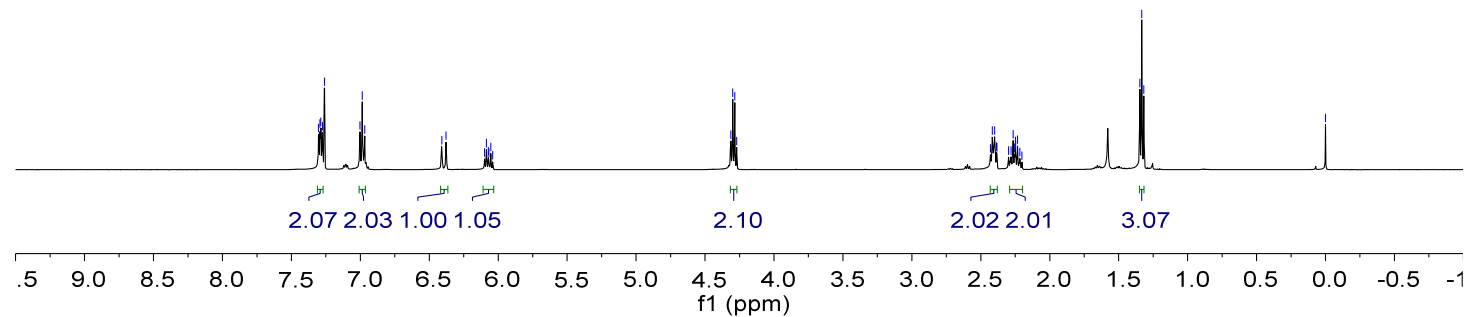




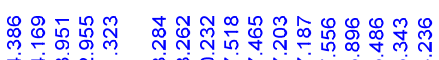

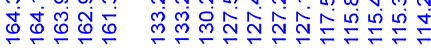

구요

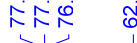<smiles>CCOC(=O)CCC/C=C/c1ccc(F)cc1</smiles>

30

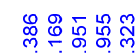

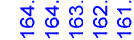

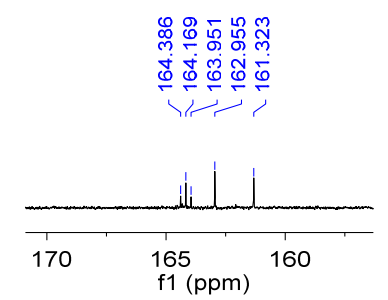

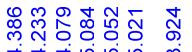

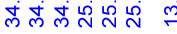

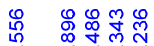

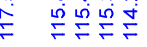

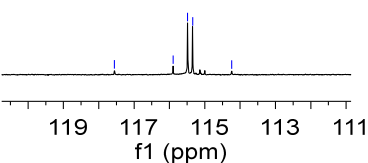

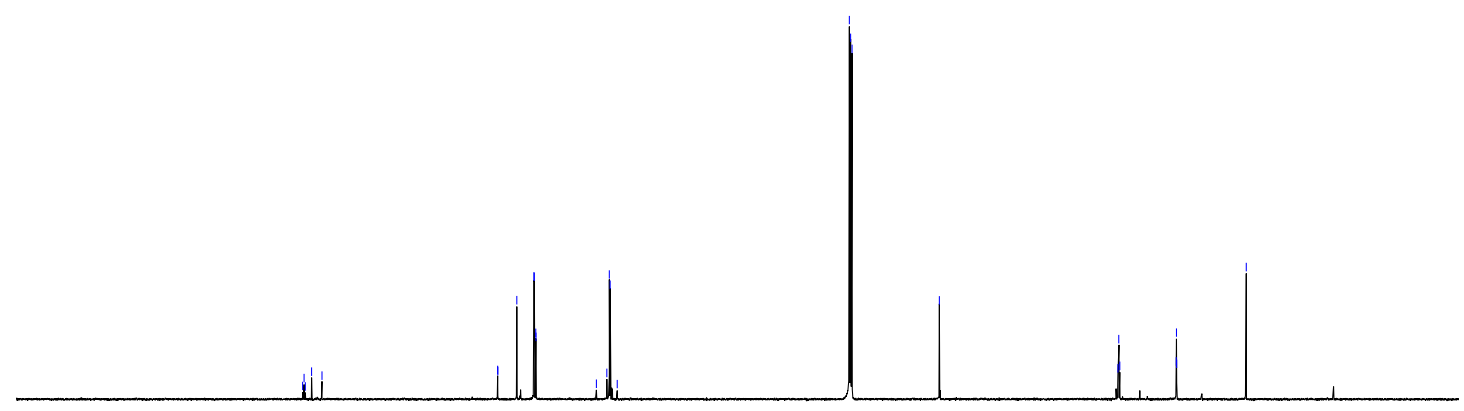

$\begin{array}{lllllllllllllllllllllll}200 & 190 & 180 & 170 & 160 & 150 & 140 & 130 & 120 & 110 & 100 & 90 & 80 & 70 & 60 & 50 & 40 & 30 & 20 & 10 & 0 & -10 & -4\end{array}$ f1 (ppm)

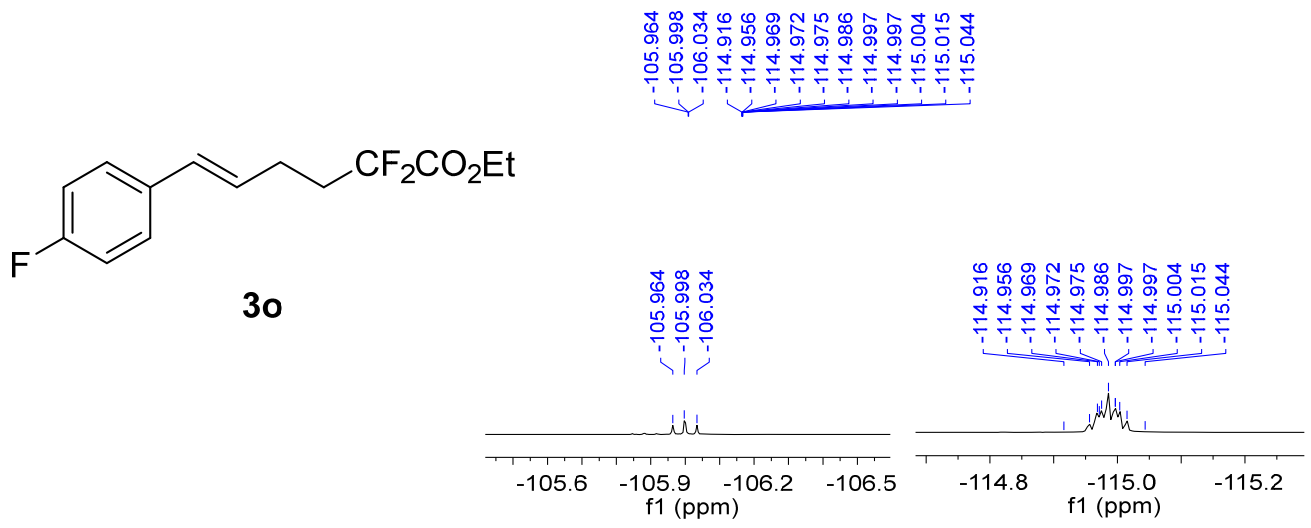

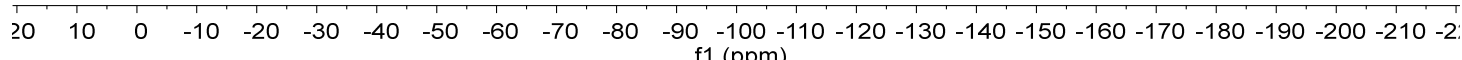


<smiles>CCOC(=O)C(F)(F)CCC/C=C/c1ccccc1F</smiles>

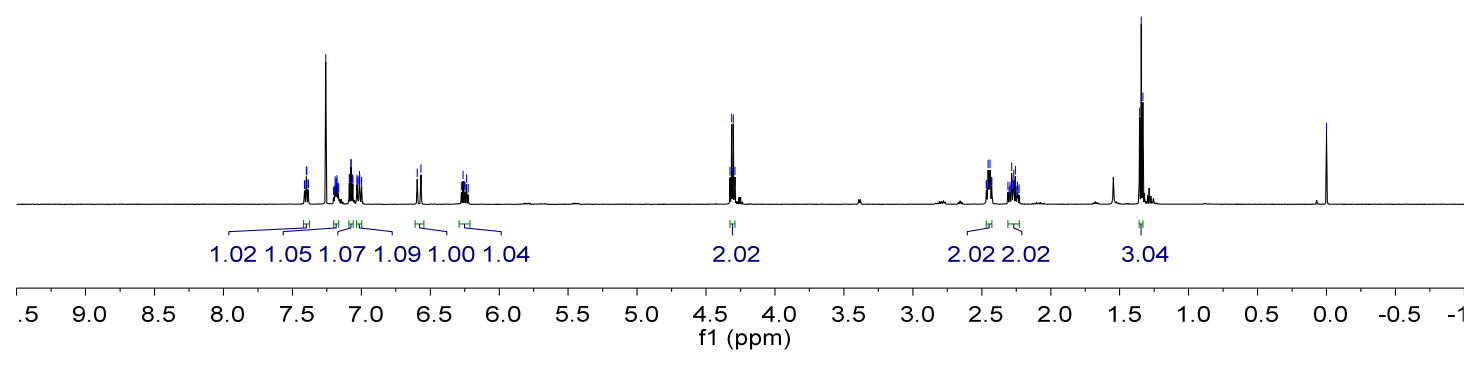

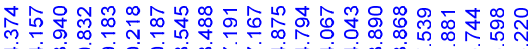

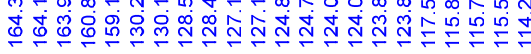<smiles>CCOC(=O)[C-](C)CC/C=C/c1ccccc1F</smiles>

$\begin{array}{lllllllllllllllllllll}190 & 180 & 170 & 160 & 150 & 140 & 130 & 120 & 110 & 100 \underset{\mathrm{f} 1}{(\mathrm{ppm})} \mathbf{9 0} & 80 & 70 & 60 & 50 & 40 & 30 & 20 & 10 & 0 & -10 & -4\end{array}$ 
<smiles>CCOC(=O)[C-](C)CCC/C=C/c1ccccc1F</smiles>

$3 p$

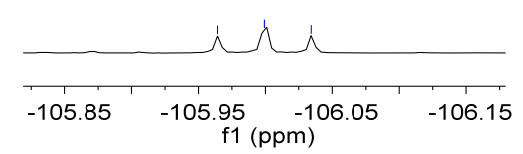

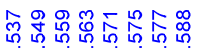

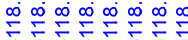

T.

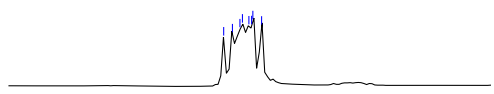

$-118.3-118.4-118.5-118.6-118.7-118.8$ f1 (ppm)<smiles>CCOC(=O)[C-]CC/C=C/c1ccc(Cl)cc1</smiles>

$3 q$

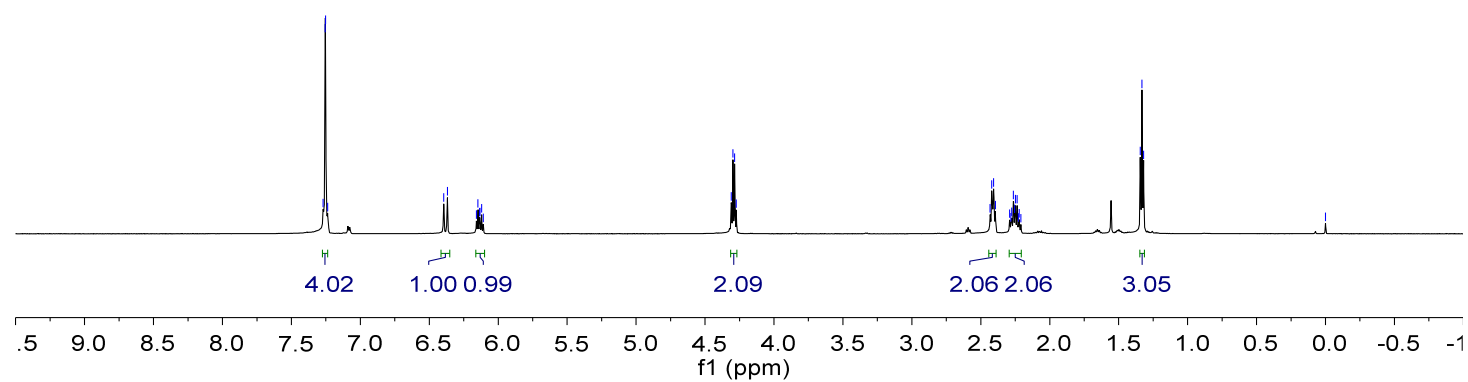



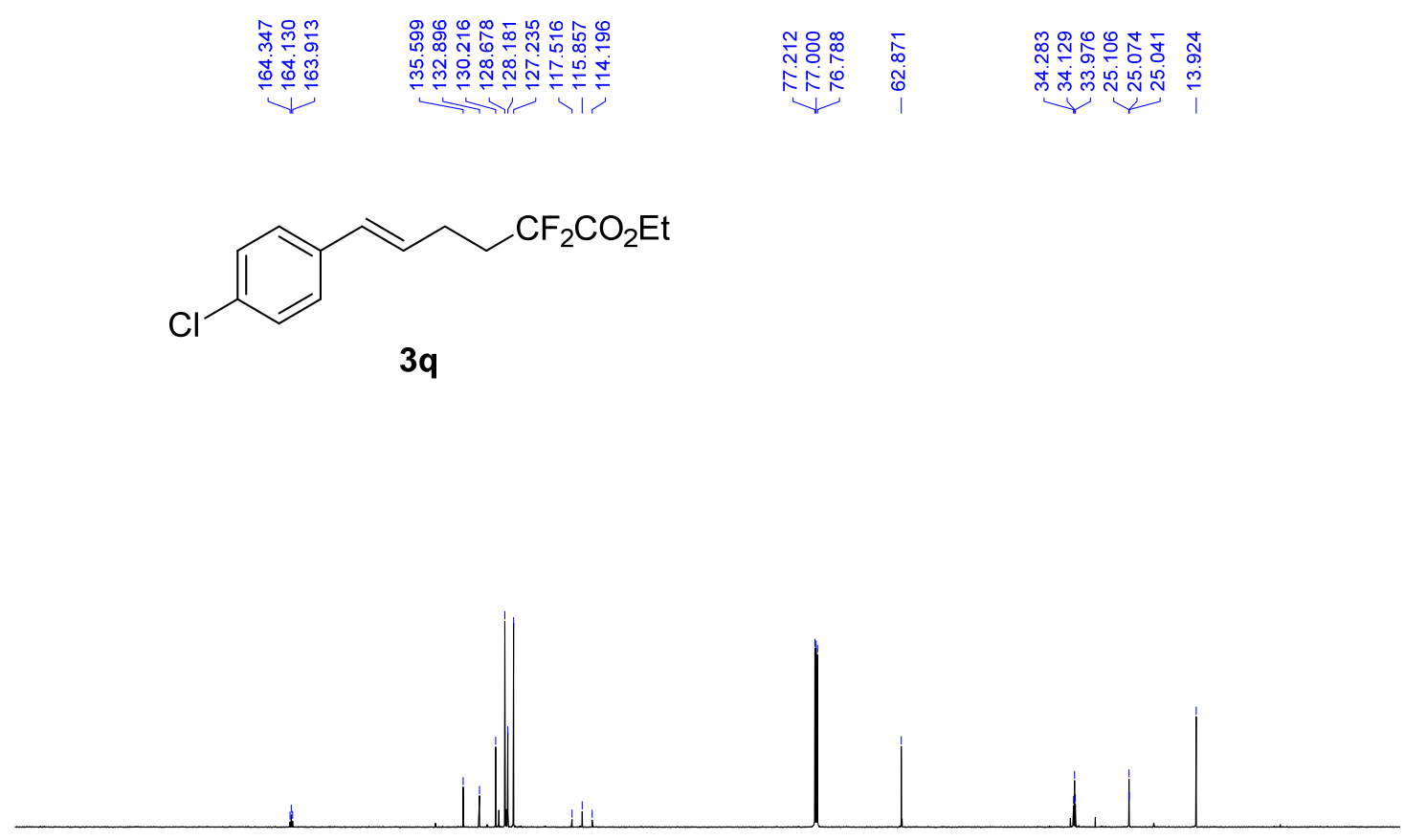

$\begin{array}{llllllllllllllllllllllllllllll}10 & 200 & 190 & 180 & 170 & 160 & 150 & 140 & 130 & 120 & 110 & \begin{array}{l}100 \\ \mathrm{f} 1(\mathrm{ppm})\end{array} & 80 & 70 & 60 & 50 & 40 & 30 & 20 & 10 & 0 & -10 & -4\end{array}$

ㅊํํํํำำำ

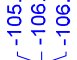<smiles>CCOC(=O)CCC/C=C/c1ccc(Cl)cc1</smiles>

층ํํㅇ

응 웅

$3 q$

$\overbrace{}^{1} 1$

$-105.80-105.90-106.00-106.10-106.20$ f1 (ppm)

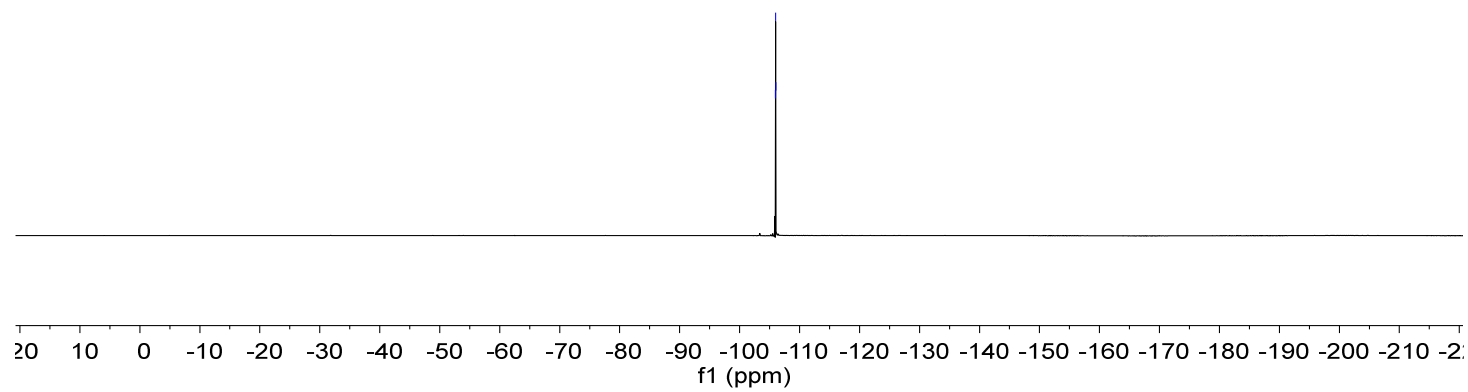


<smiles>CCOC(=O)CCC/C=C/c1ccc(Cl)c(Cl)c1</smiles>

$3 r$

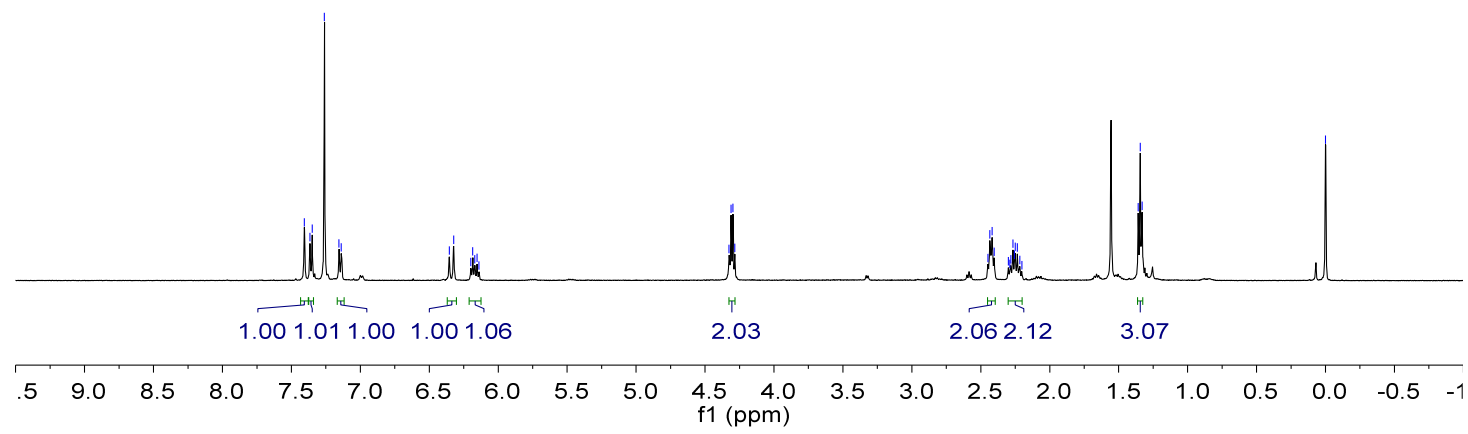

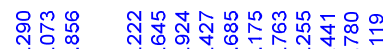

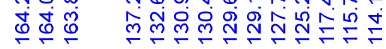

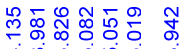

i

पा लmखñ

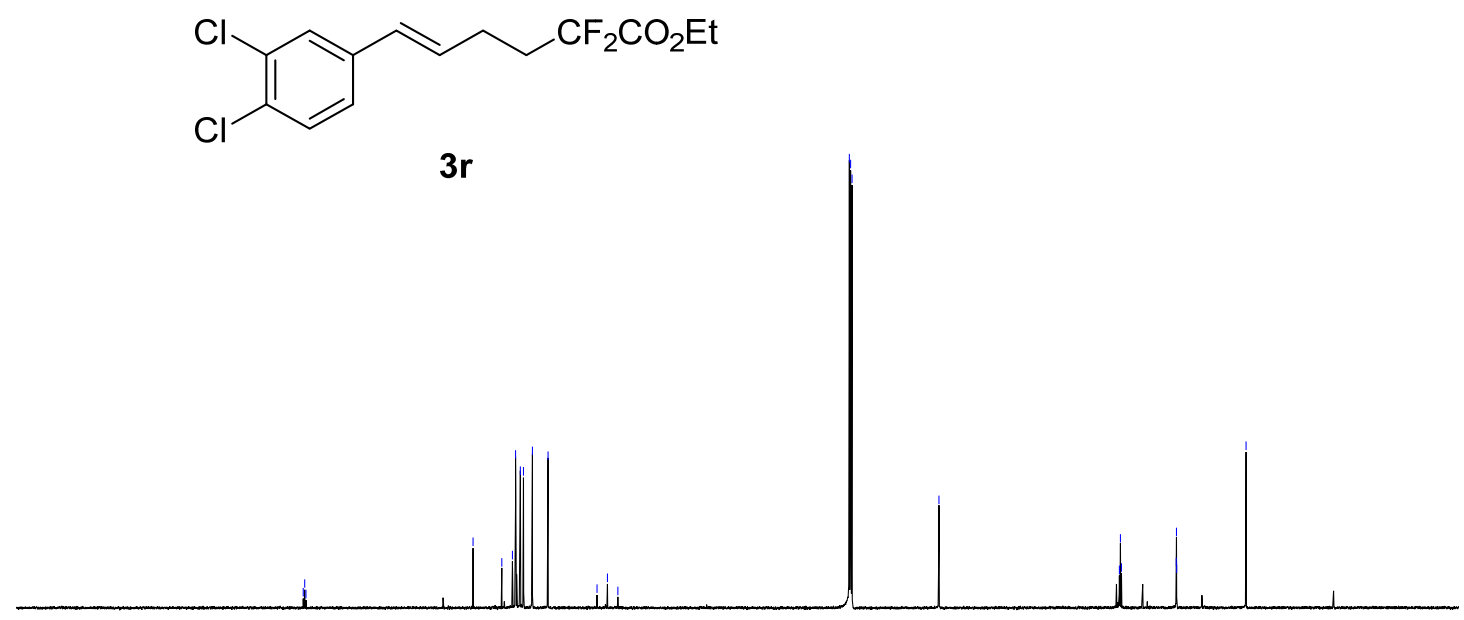

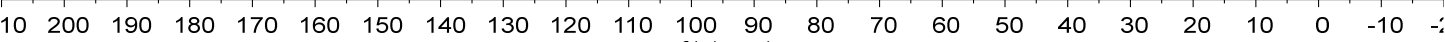

f1 (ppm) 


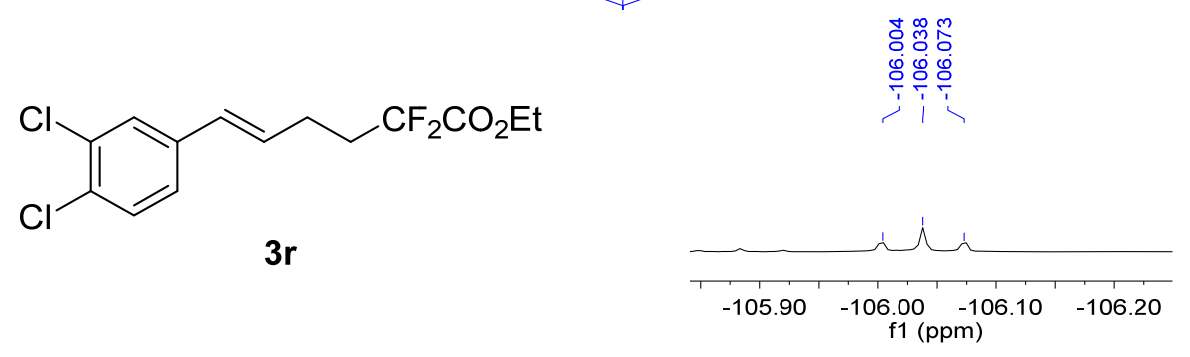

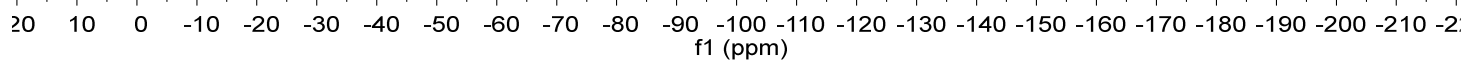

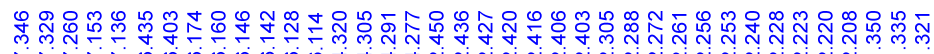

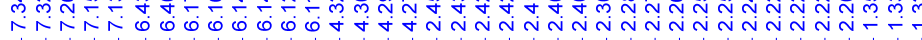
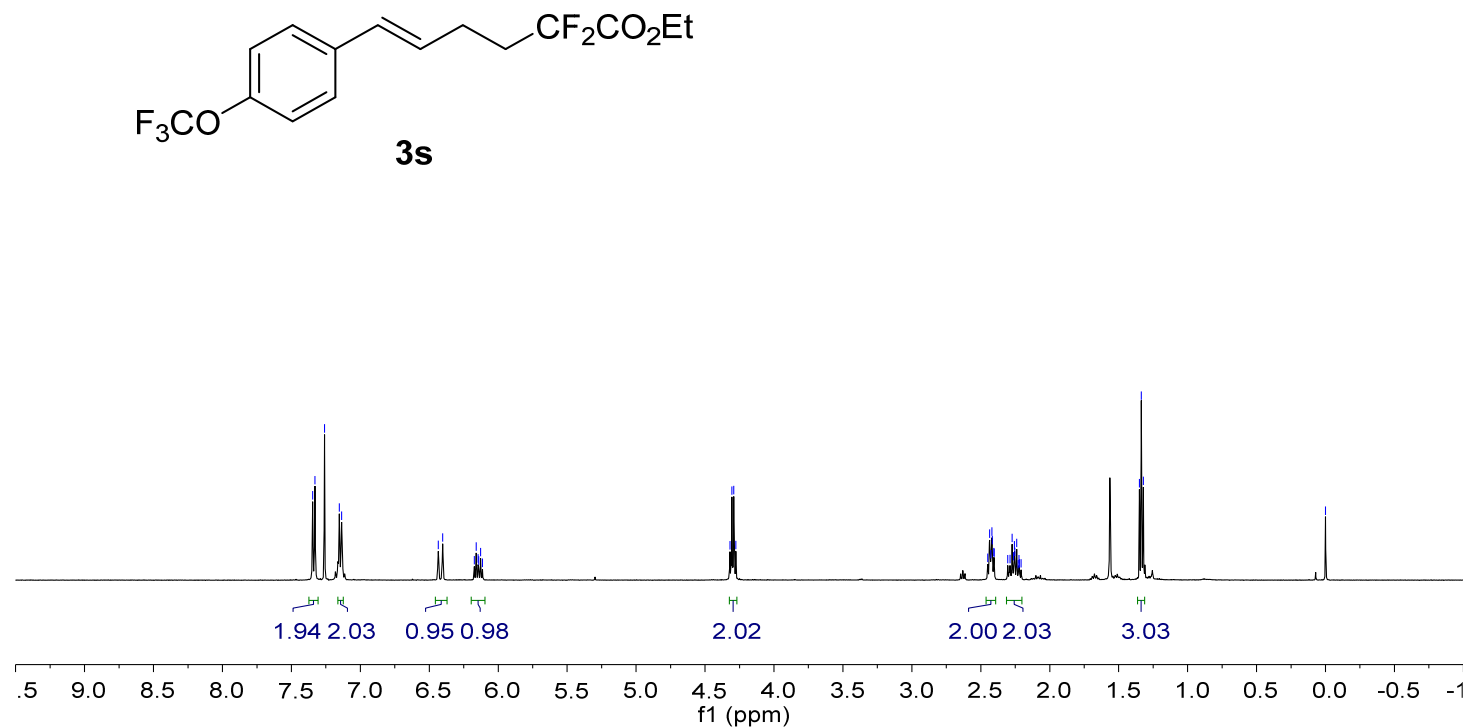


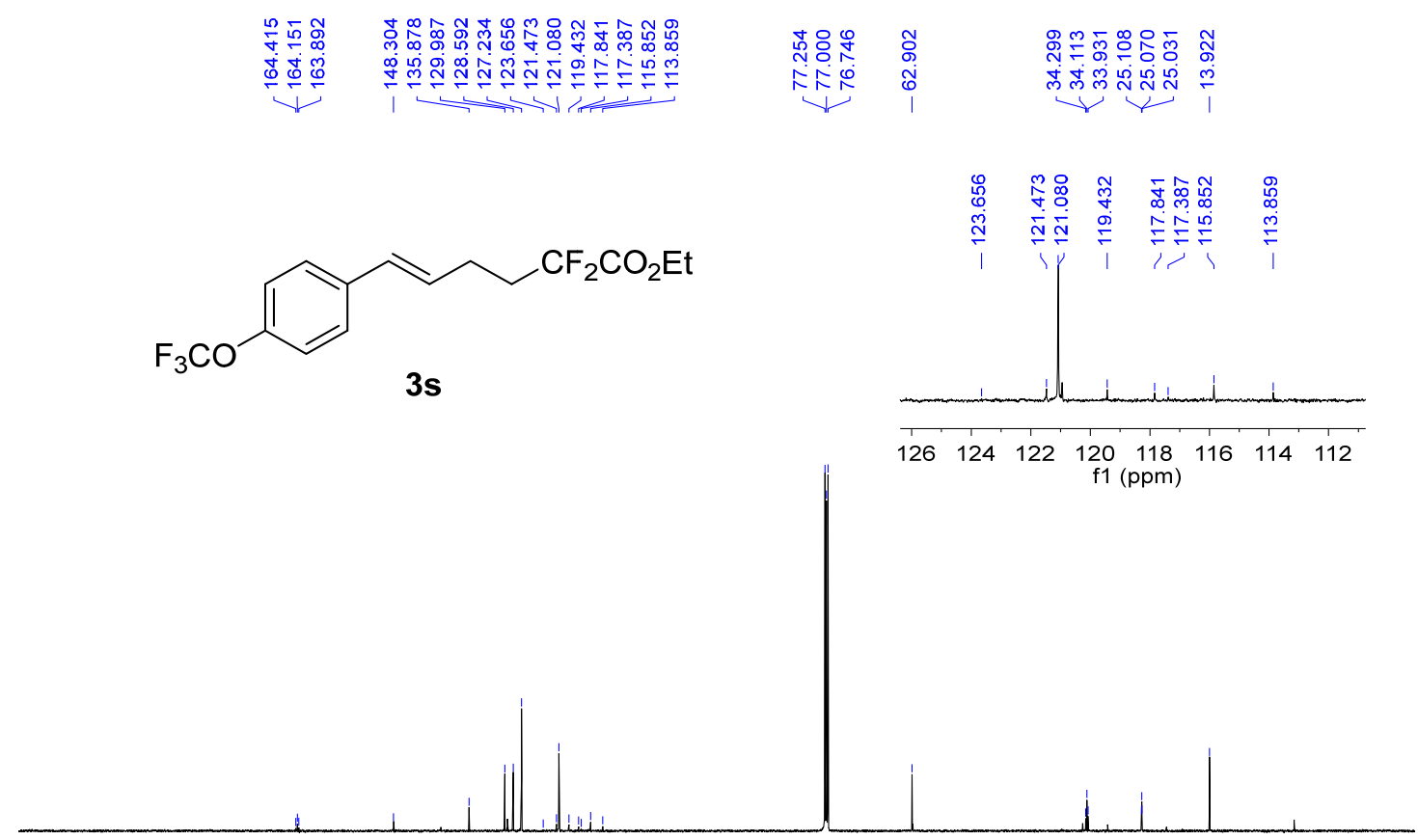

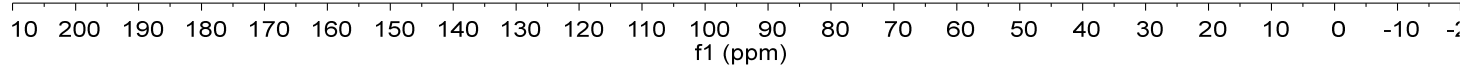
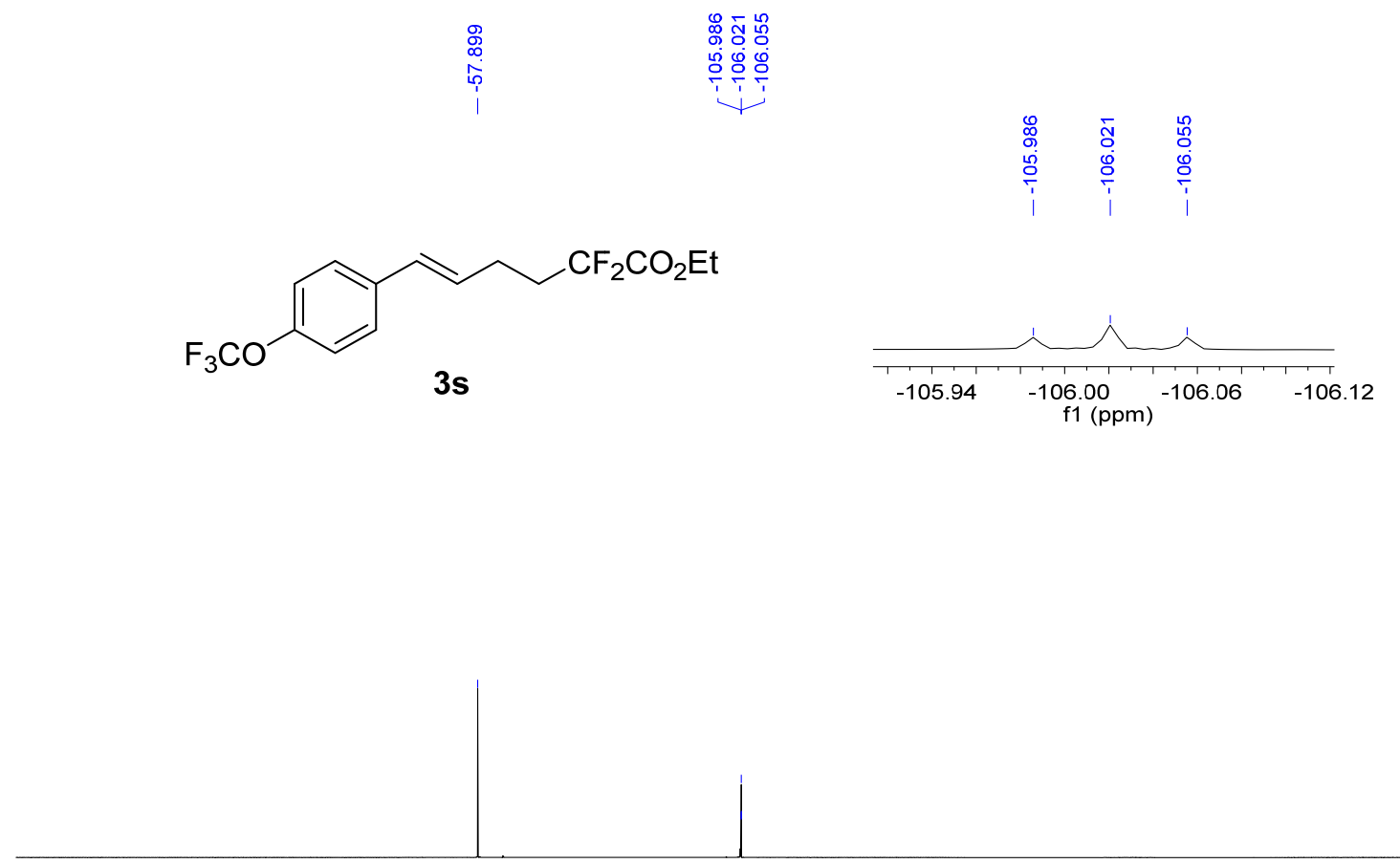

$2010 \quad 0 \quad-10 \quad-20 \quad-30 \quad-40 \quad-50 \quad-60 \quad-70 \quad-80 \quad-90-100-110-120-130-140-150-160-170-180-190-200-210-220$ 
<smiles></smiles>

$3 t$

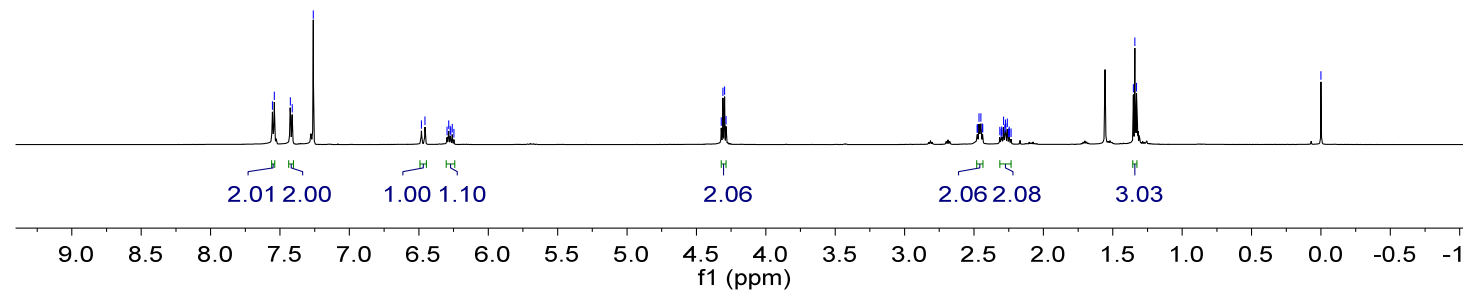

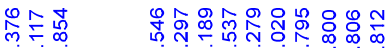

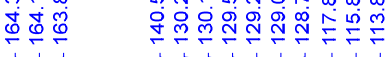

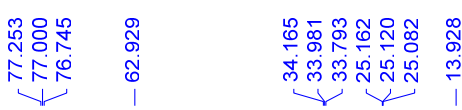

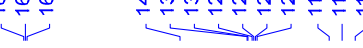
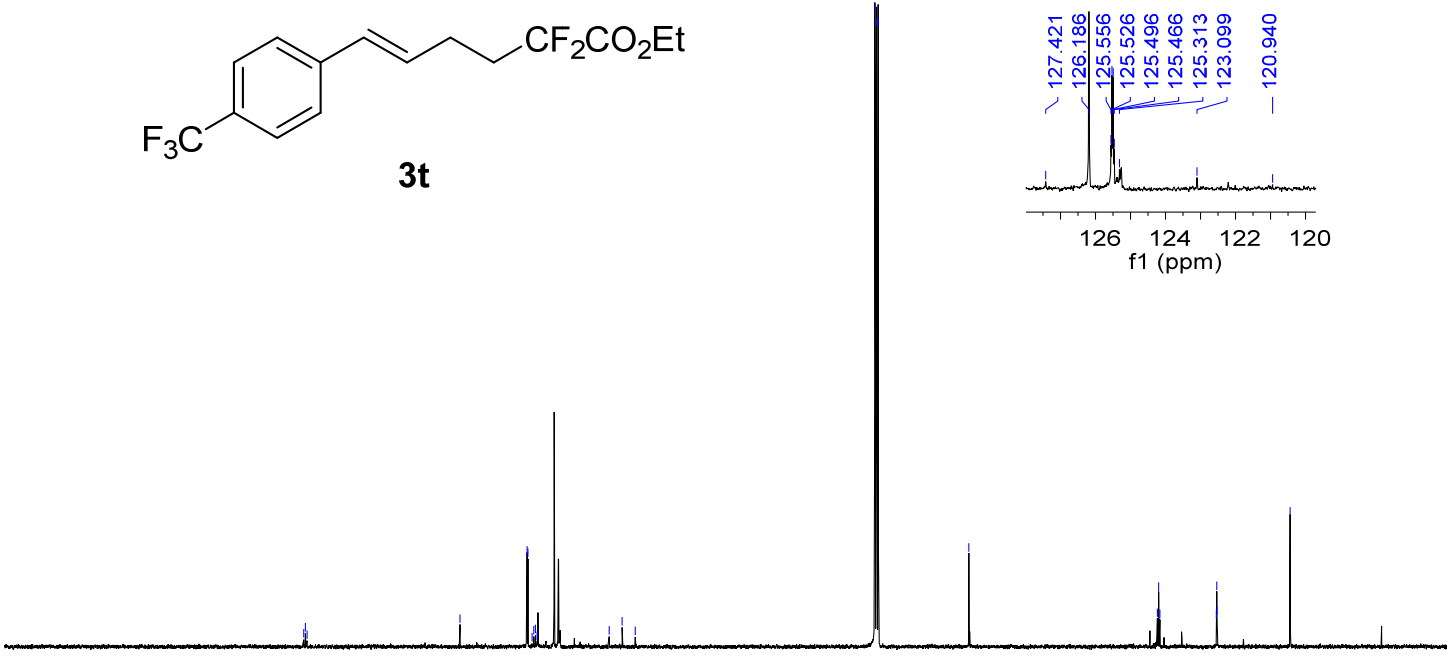

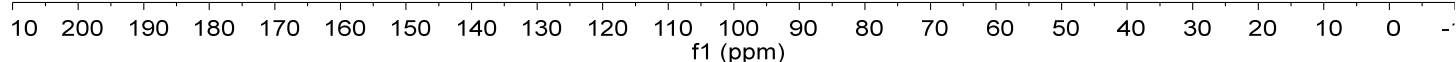



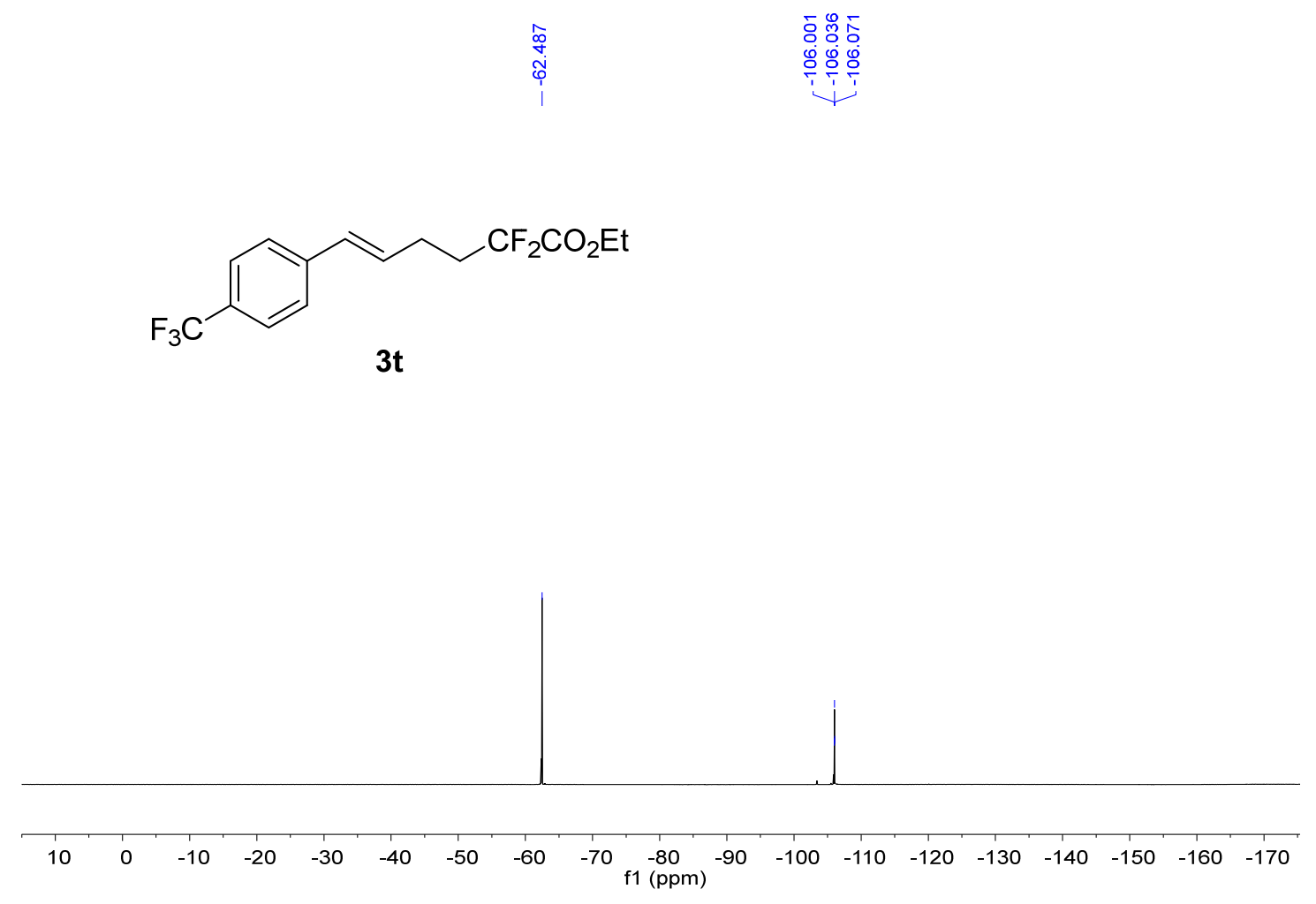

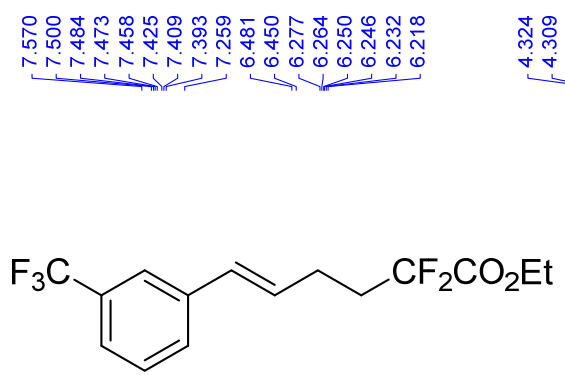

$3 u$

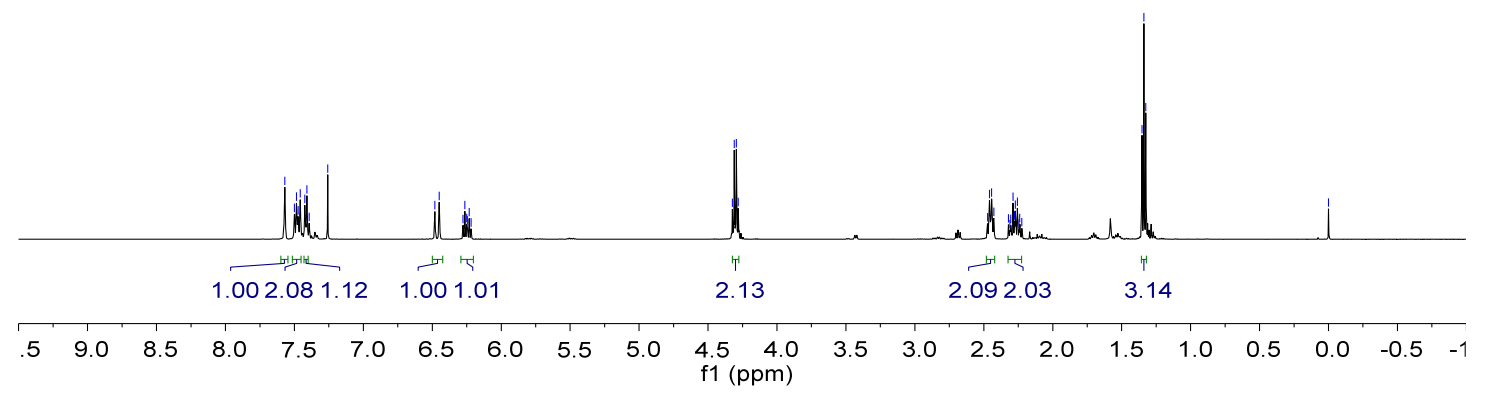




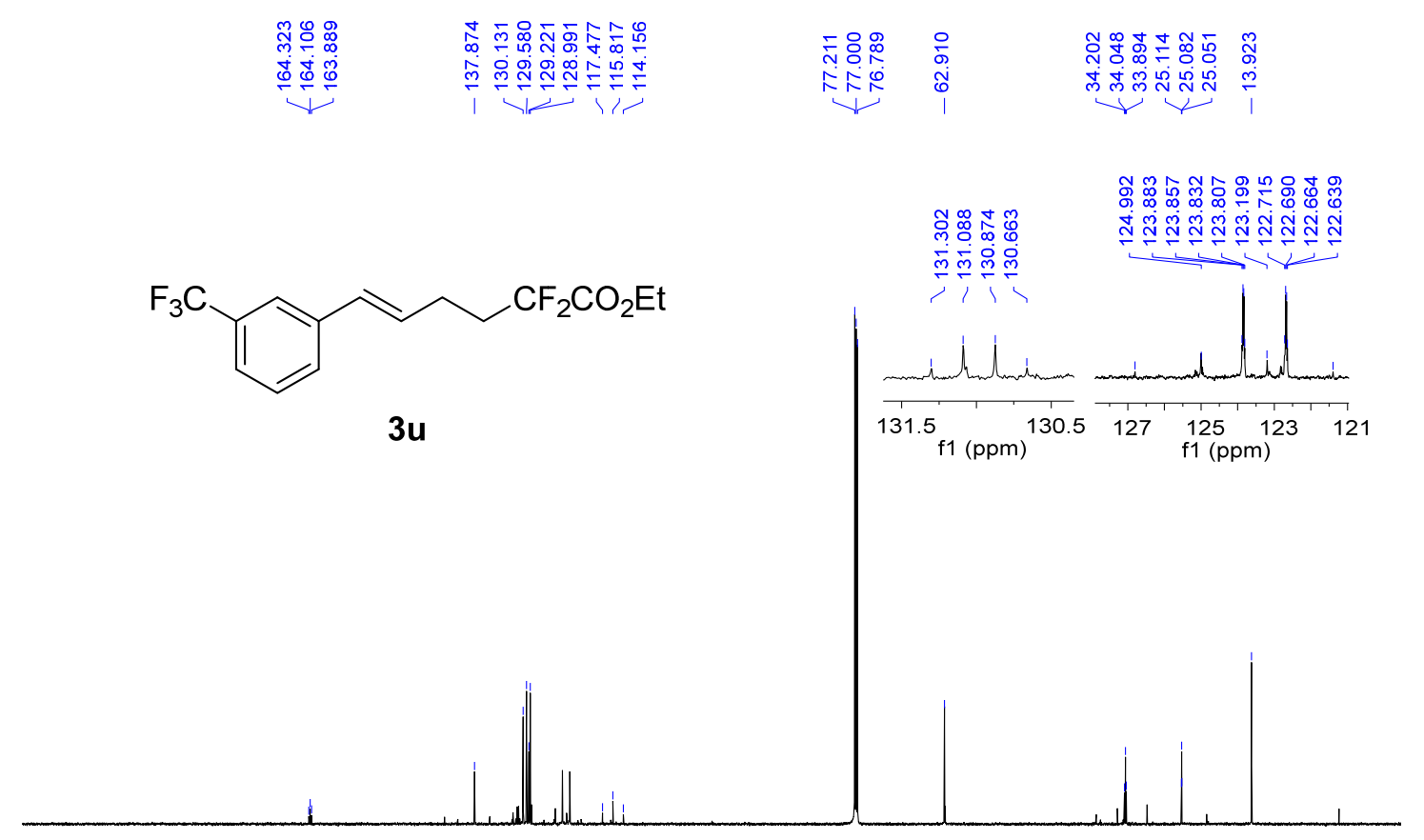

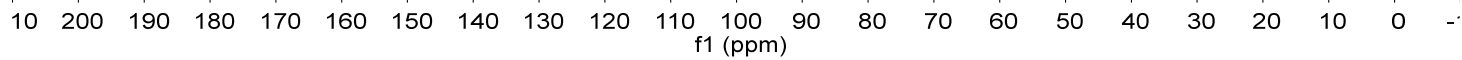

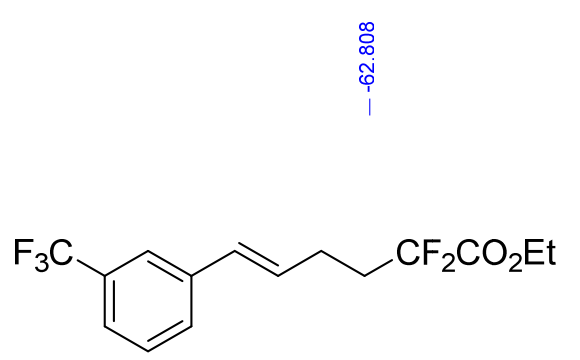

$3 u$

为商商

웅

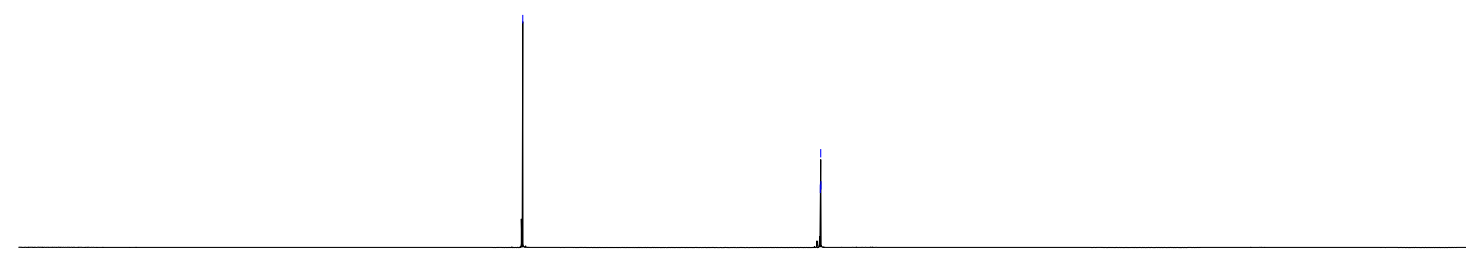

$\begin{array}{lllllllllllllllllllllll} & 0 & 0 & -10 & -20 & -30 & -40 & -50 & -60 & -70 & -80 & -90 & -100 & -110 & -120 & -130 & -140 & -150 & -160 & -170 & -180 & -190\end{array}$ 


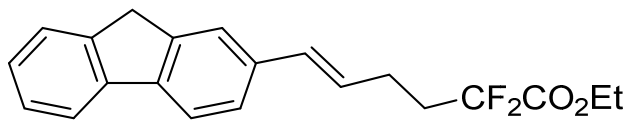

$3 v$

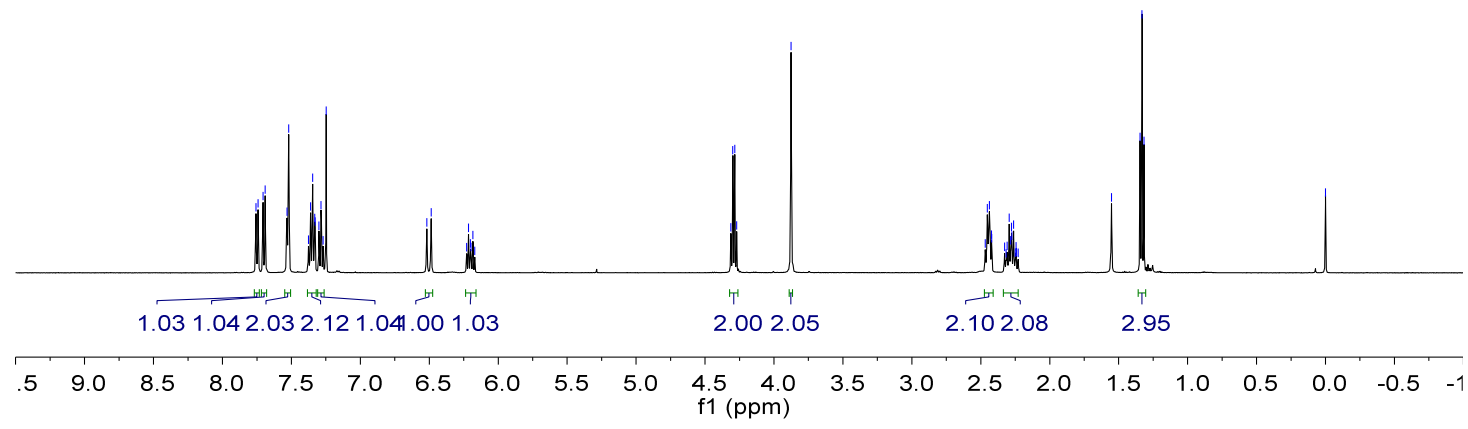

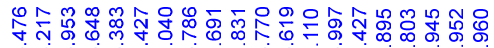

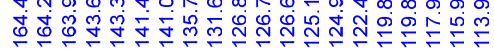

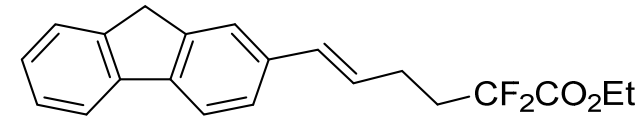

$3 v$

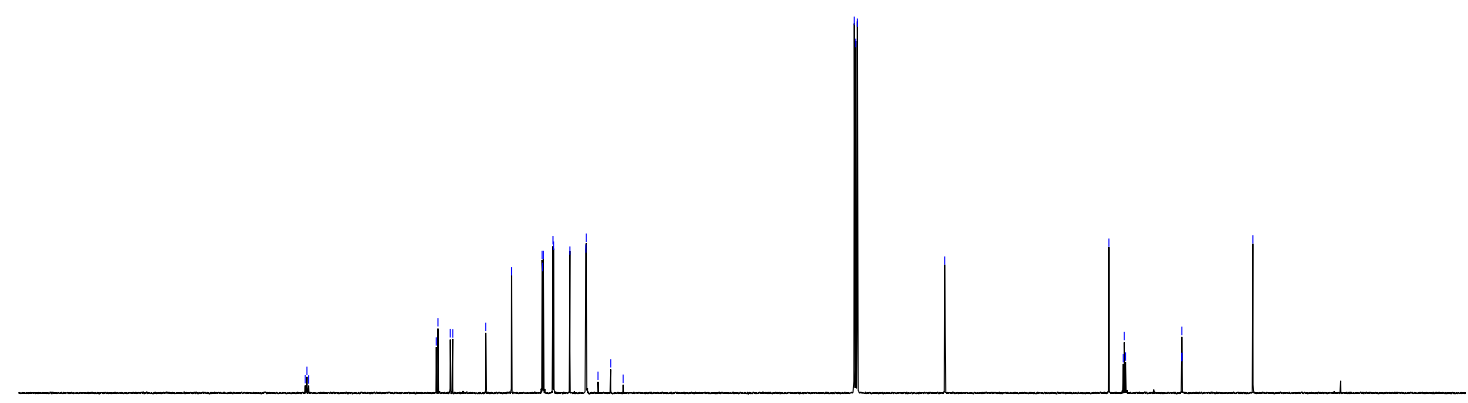

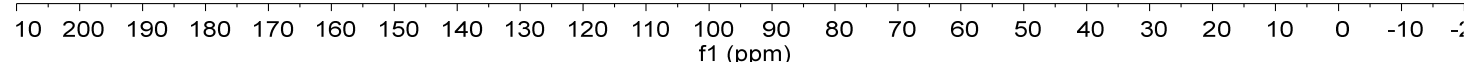



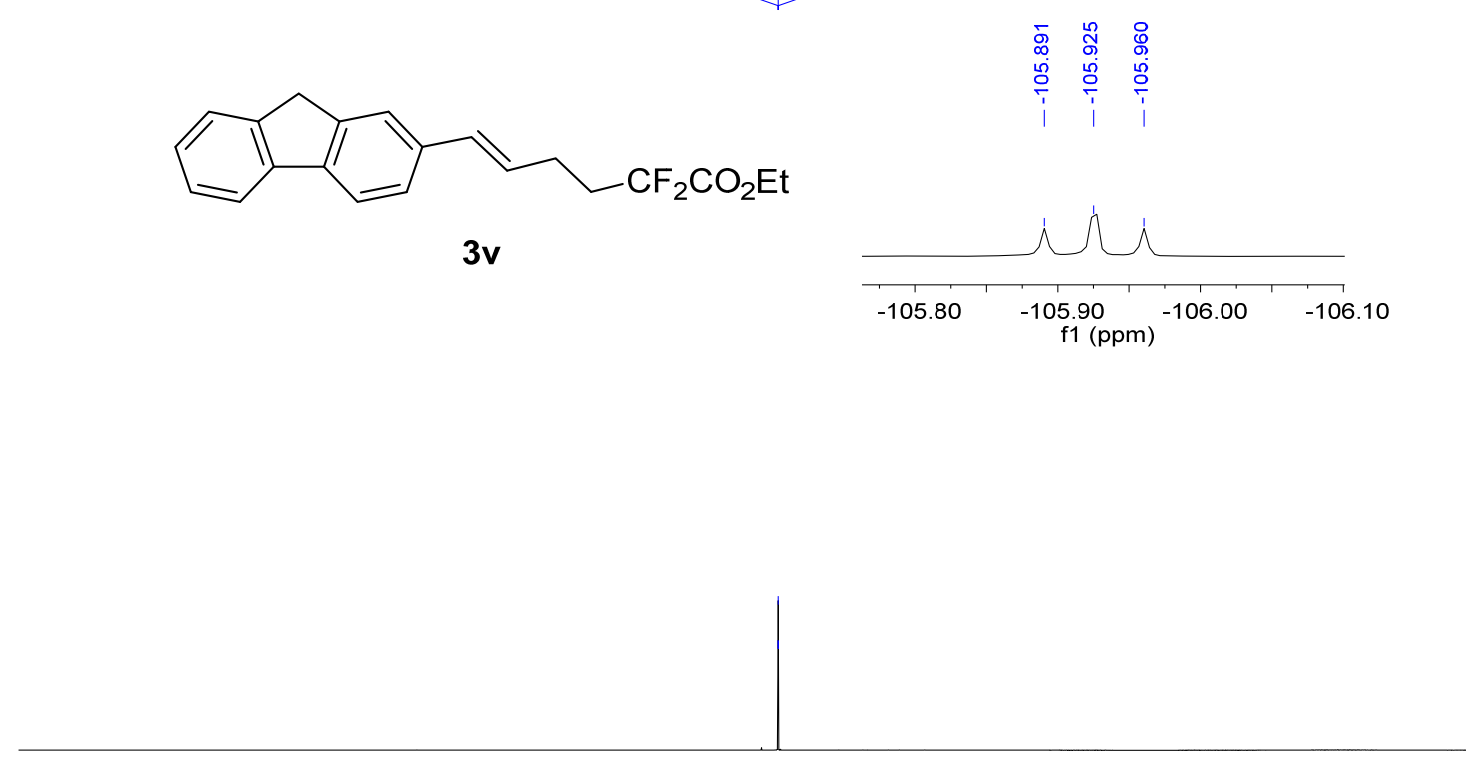

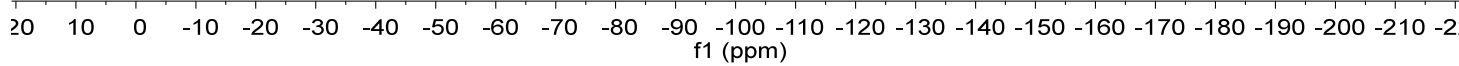

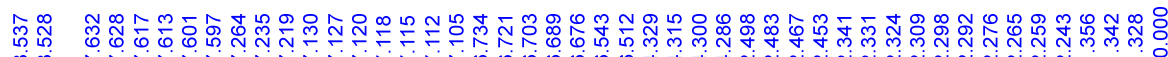

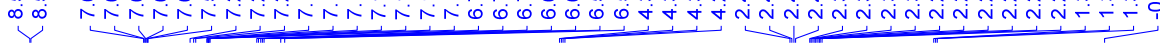

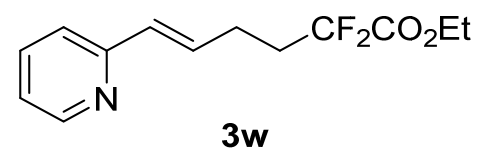

$3 w$

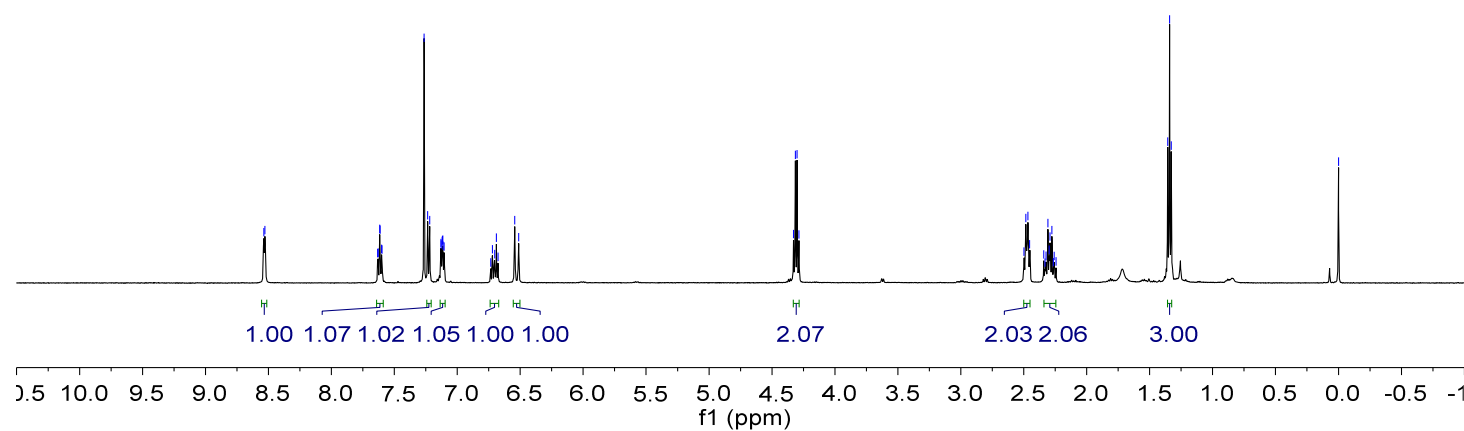




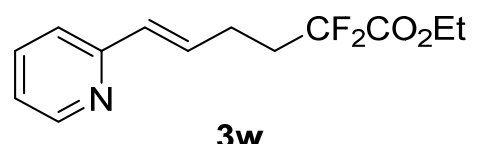

$3 w$

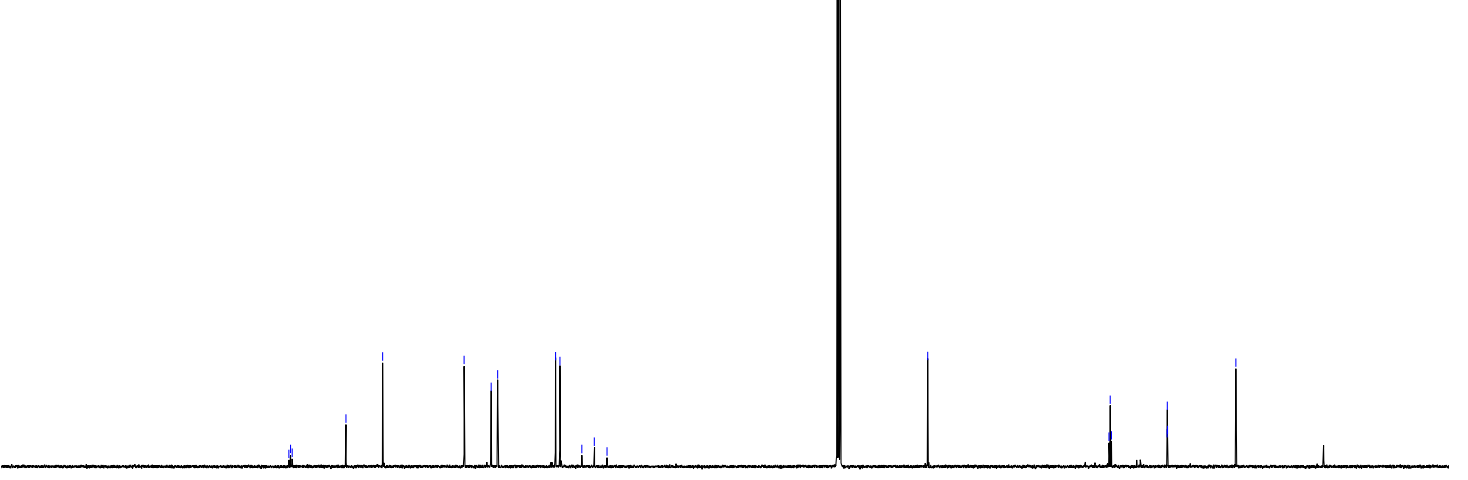

$\begin{array}{lllllllllllllllllllllll}10 & 200 & 190 & 180 & 170 & 160 & 150 & 140 & 130 & 120 & 110 & \begin{array}{l}100 \\ \mathrm{f} 1(\mathrm{ppm})\end{array} & 80 & 70 & 60 & 50 & 40 & 30 & 20 & 10 & 0 & -10 & -:\end{array}$

\section{$\stackrel{\infty}{\circ)} \stackrel{\infty}{\frac{\infty}{ \pm}}$}

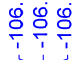

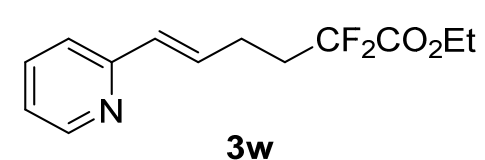

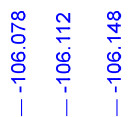

$3 w$

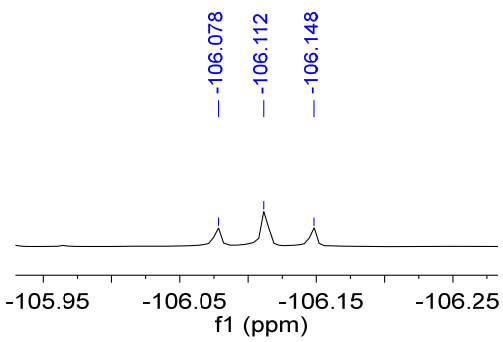

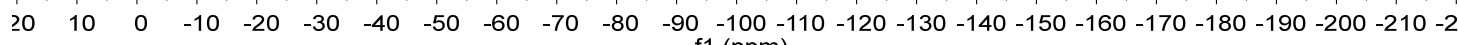
f1 (ppm) 

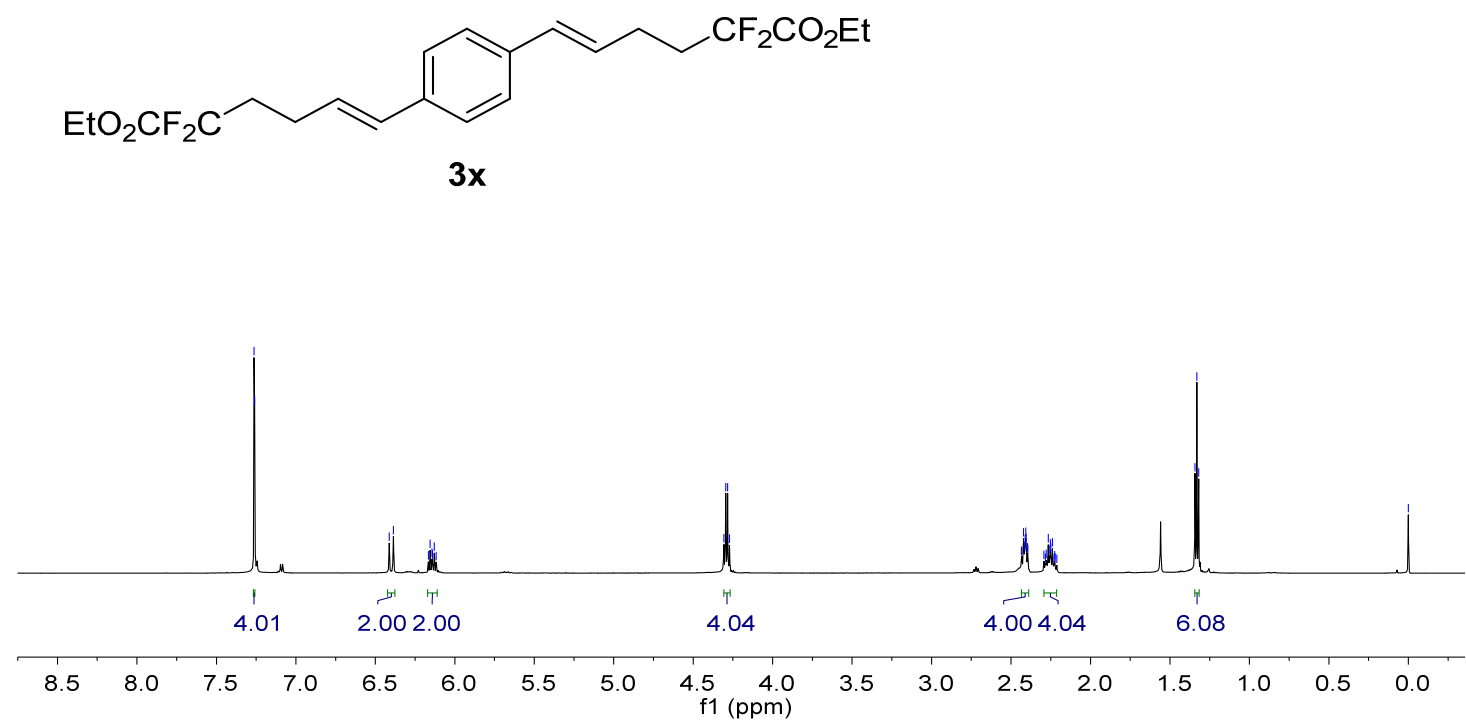

\begin{tabular}{|c|c|c|c|c|}
\hline 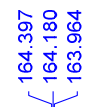 & 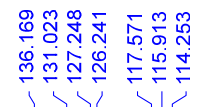 & 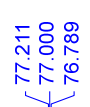 & $\begin{array}{l}\text { fo } \\
\text { o } \\
\text { ij } \\
0 \\
1\end{array}$ & 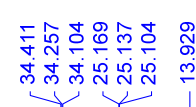 \\
\hline
\end{tabular}<smiles>CCOC(=O)CCCC=Cc1ccc(C=CCCCC(OCC)OCC)cc1</smiles>

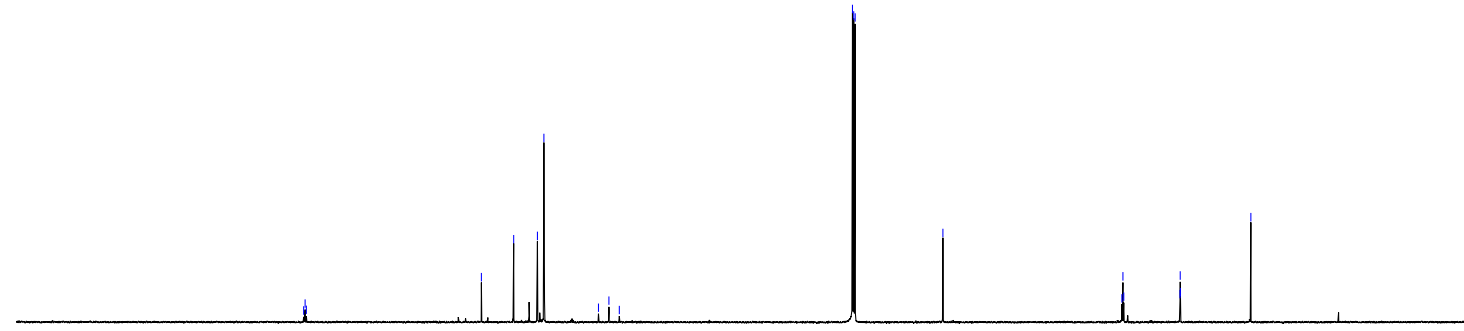

$\begin{array}{llllllllllllllllllllllllll}10 & 200 & 190 & 180 & 170 & 160 & 150 & 140 & 130 & 120 & 110 & \begin{array}{r}100 \\ \mathrm{f} 1(\mathrm{ppm})\end{array} & 80 & 70 & 60 & 50 & 40 & 30 & 20 & 10 & 0 & -10 & -:\end{array}$ 

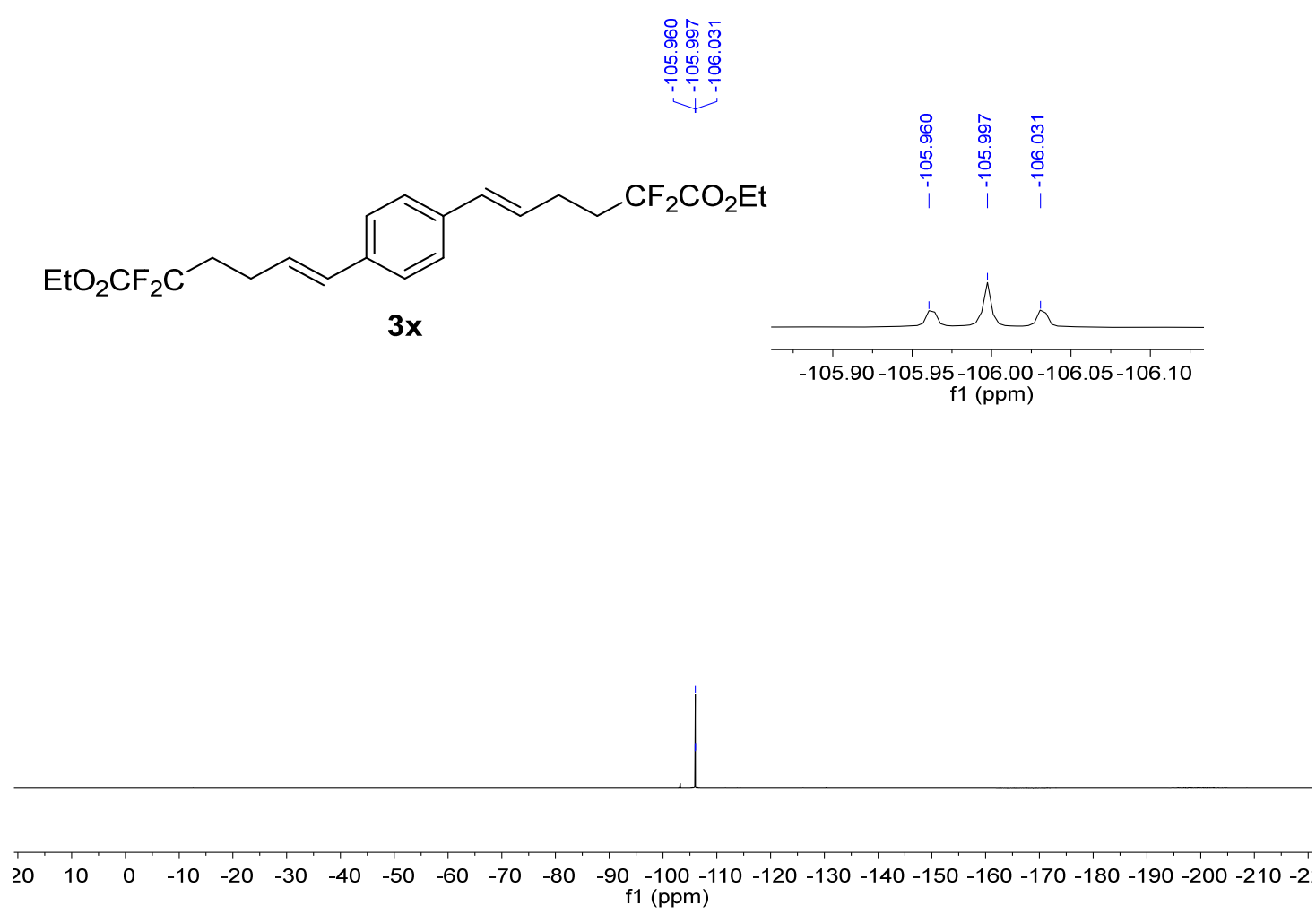

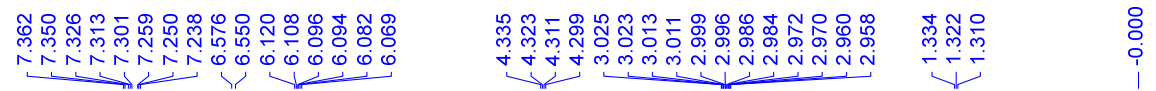

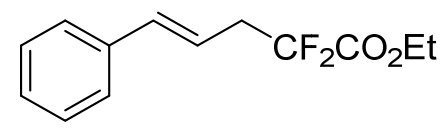

$3 y$

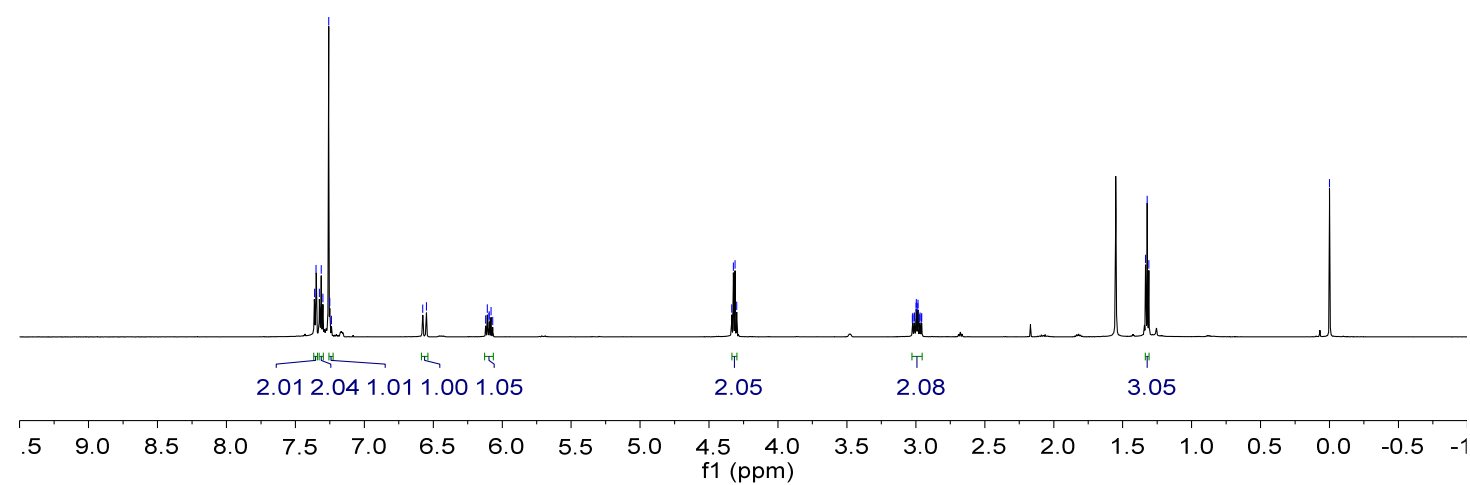




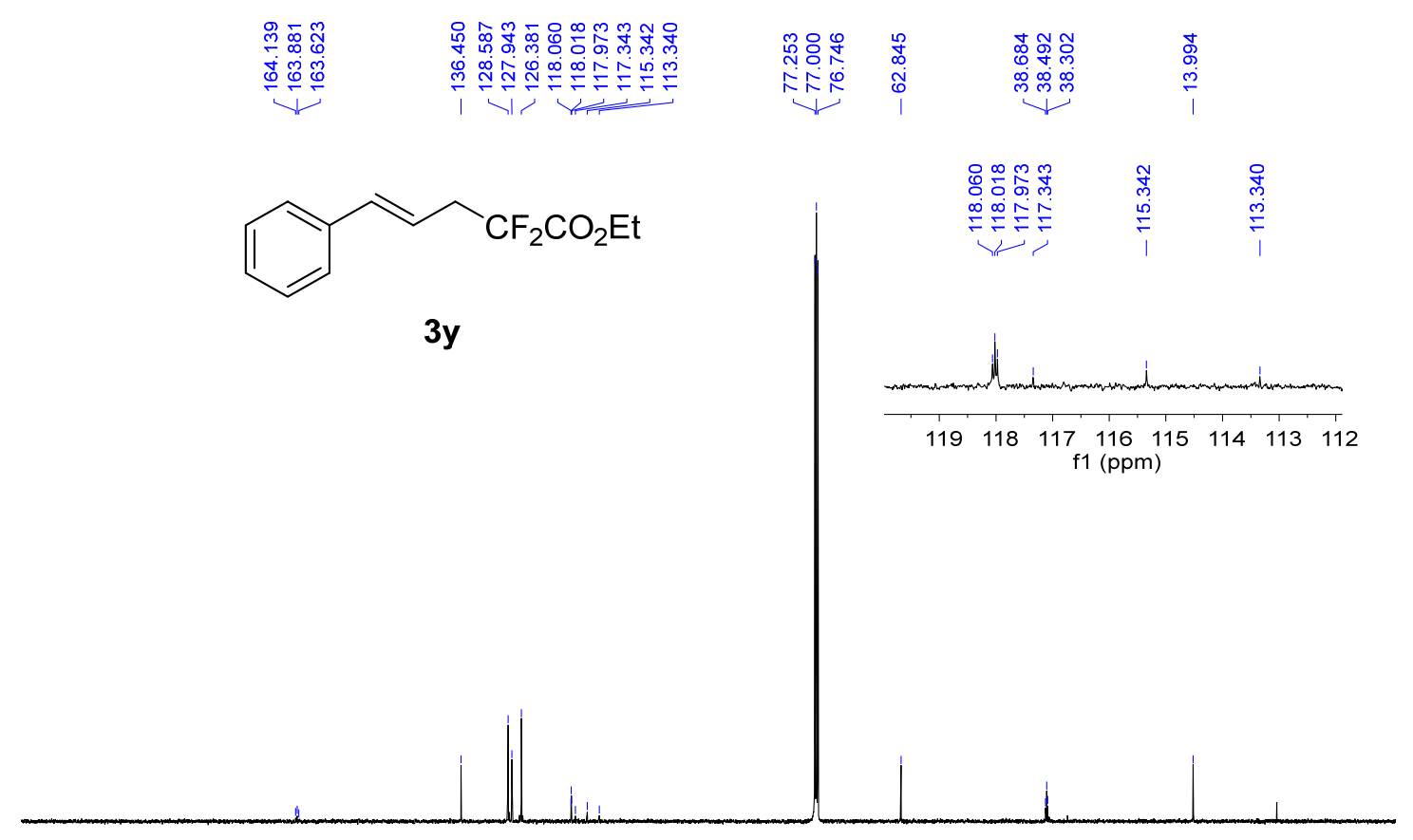

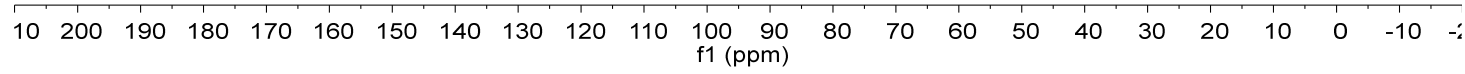

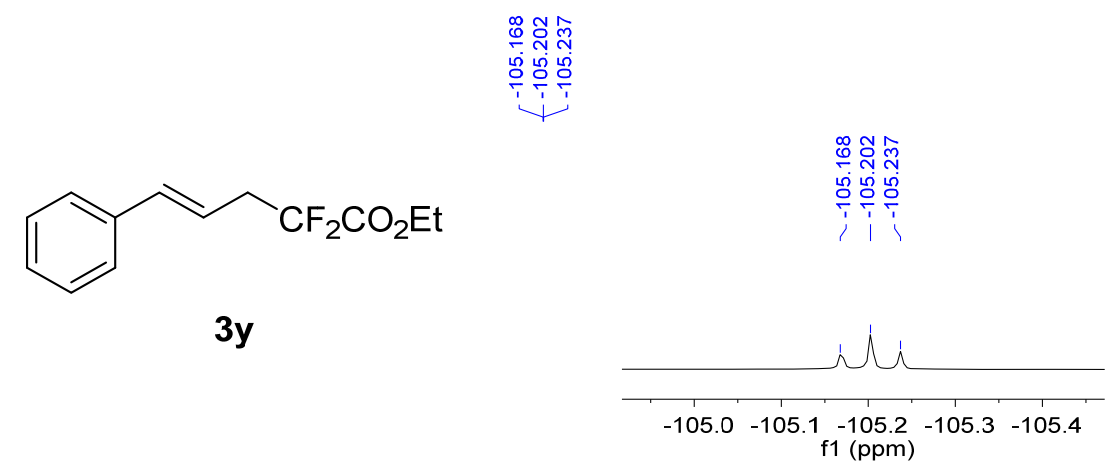

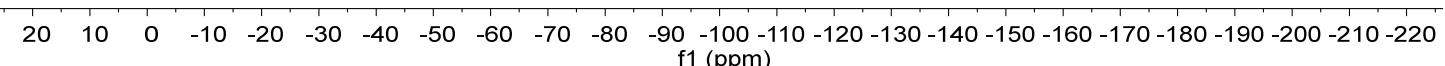




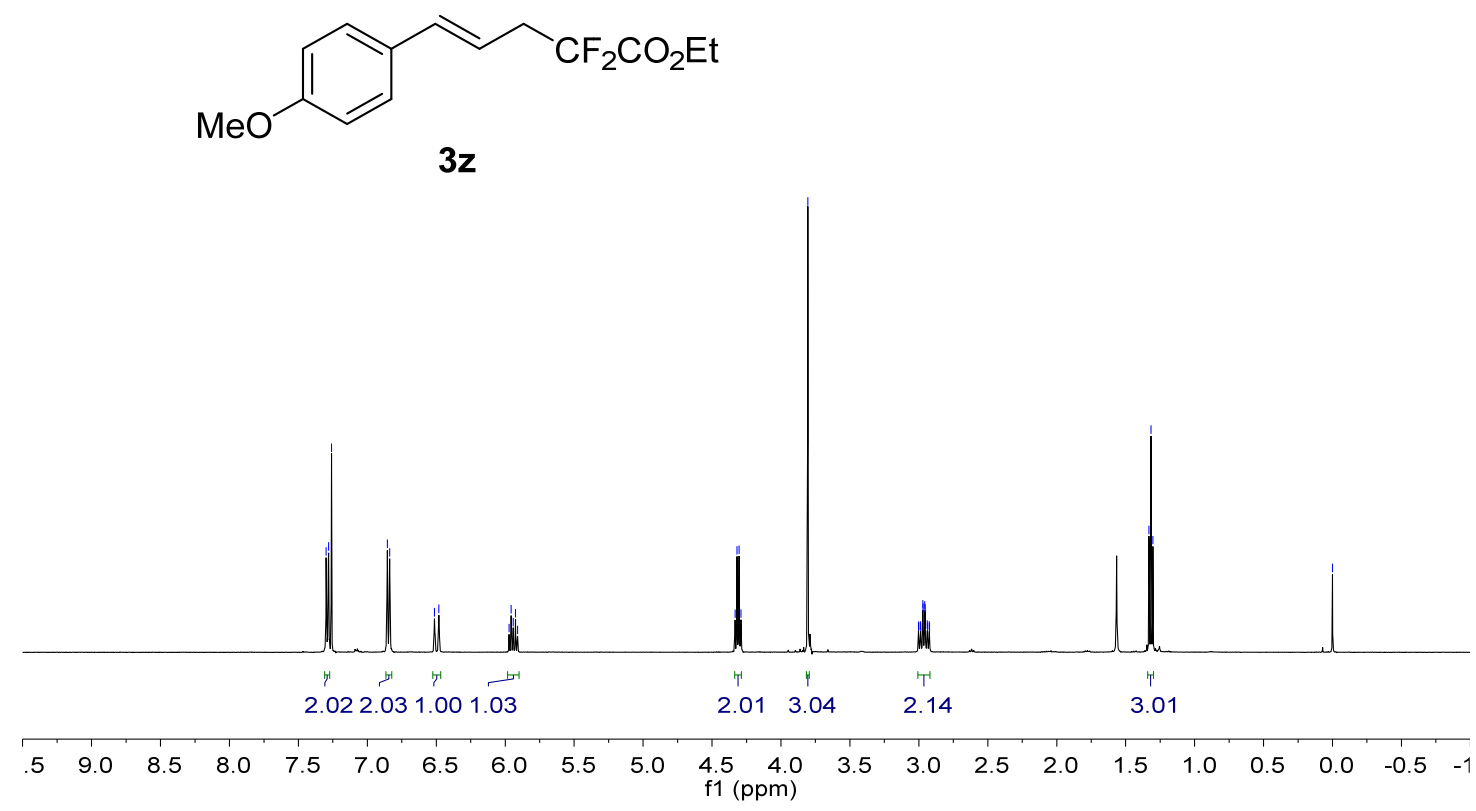

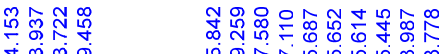

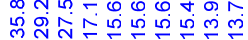

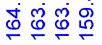

$1<1$

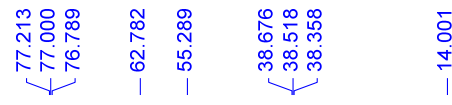

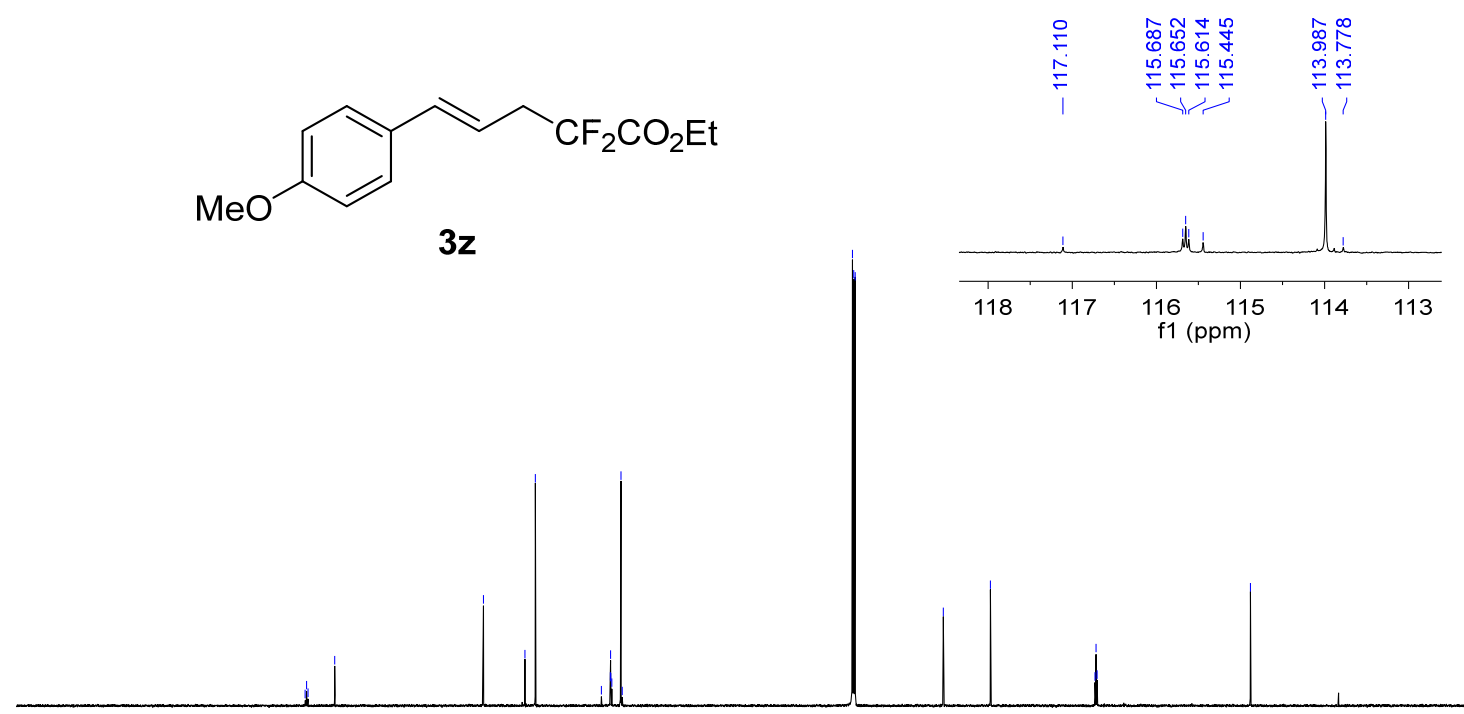

$\begin{array}{lllllllllllll}10 & 200 & 190 & 180 & 170 & 160 & 150 & 140 & 130 & 120 & 110 & 100 & 90\end{array}$ 


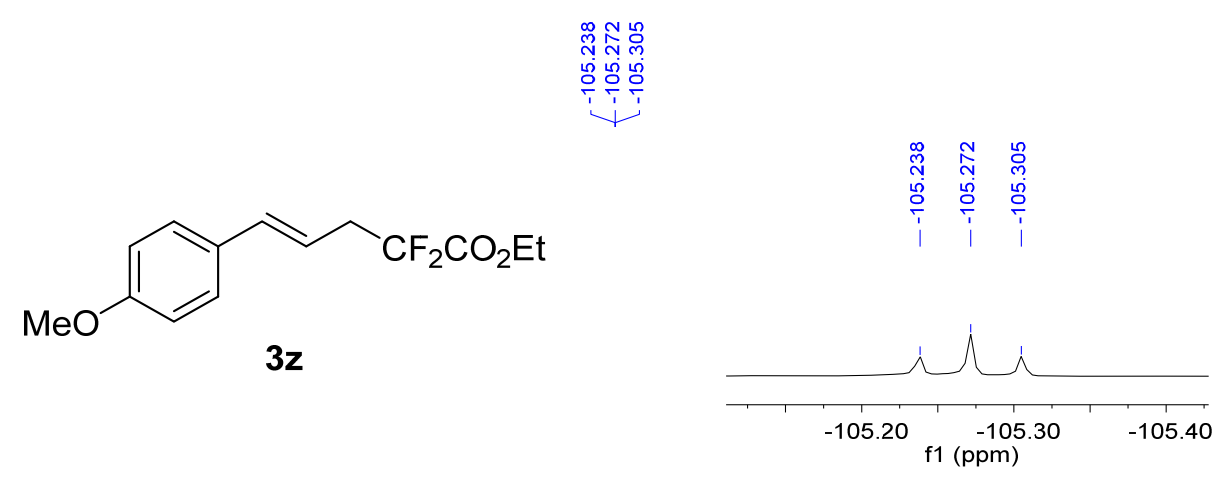

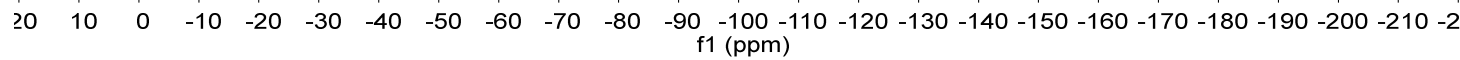

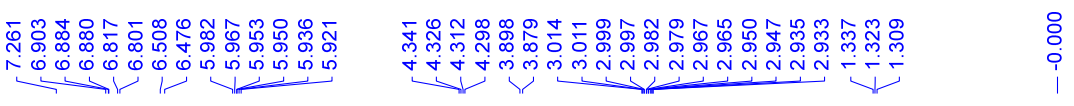<smiles>CCOC(=O)CC/C=C/c1ccc(OC)c(OC)c1</smiles>

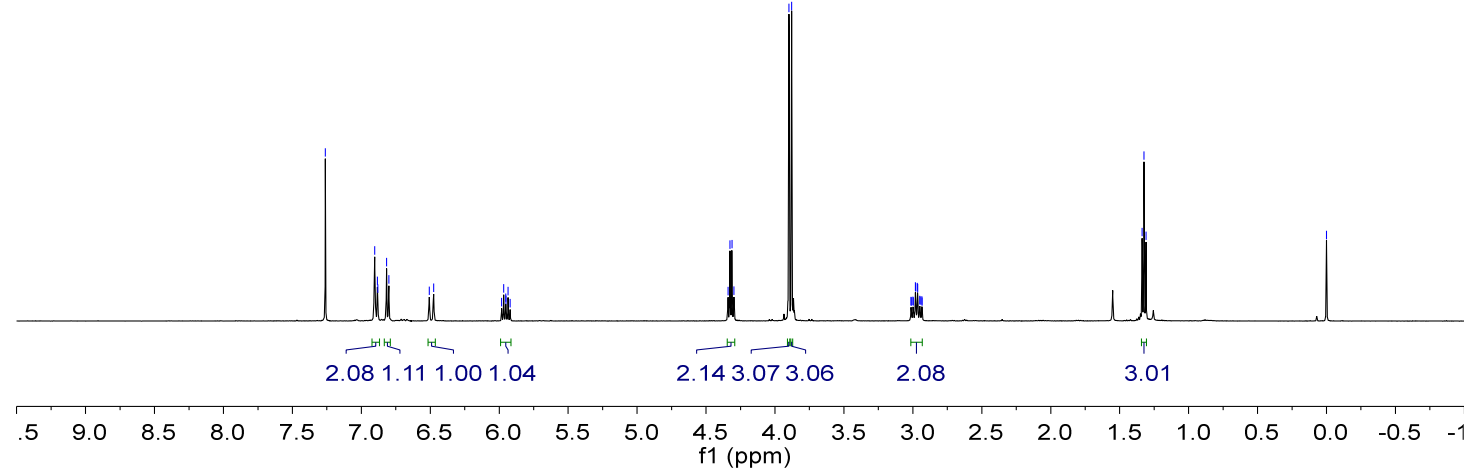




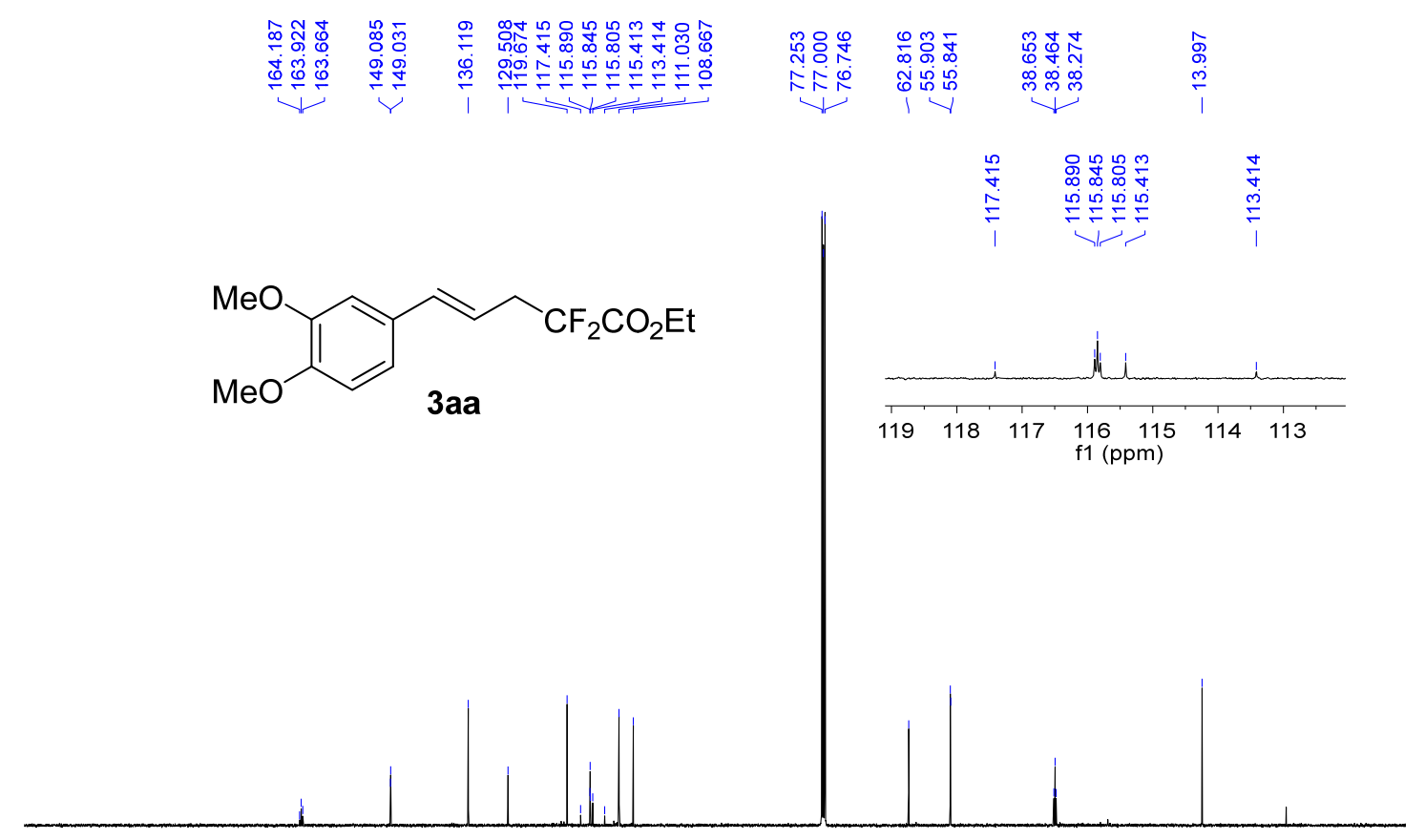

$\begin{array}{lllllllllllllllllllllllllllllll}10 & 200 & 190 & 180 & 170 & 160 & 150 & 140 & 130 & 120 & 110 & \begin{array}{c}100 \\ \mathrm{f} 1(\mathrm{ppm})\end{array} & 80 & 70 & 60 & 50 & 40 & 30 & 20 & 10 & 0 & -10 & -4\end{array}$

욱 웜

웅 웅

i

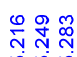

응 웅음

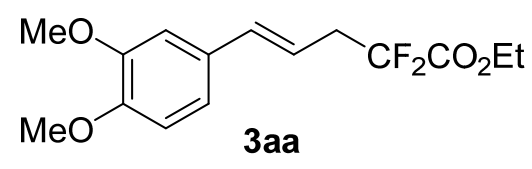

i i i

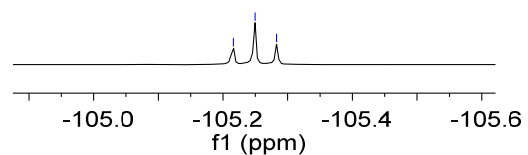

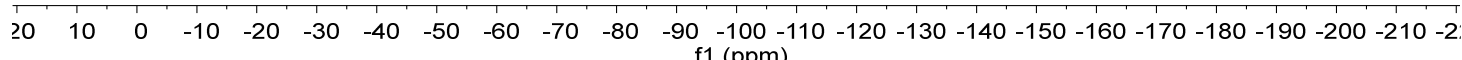




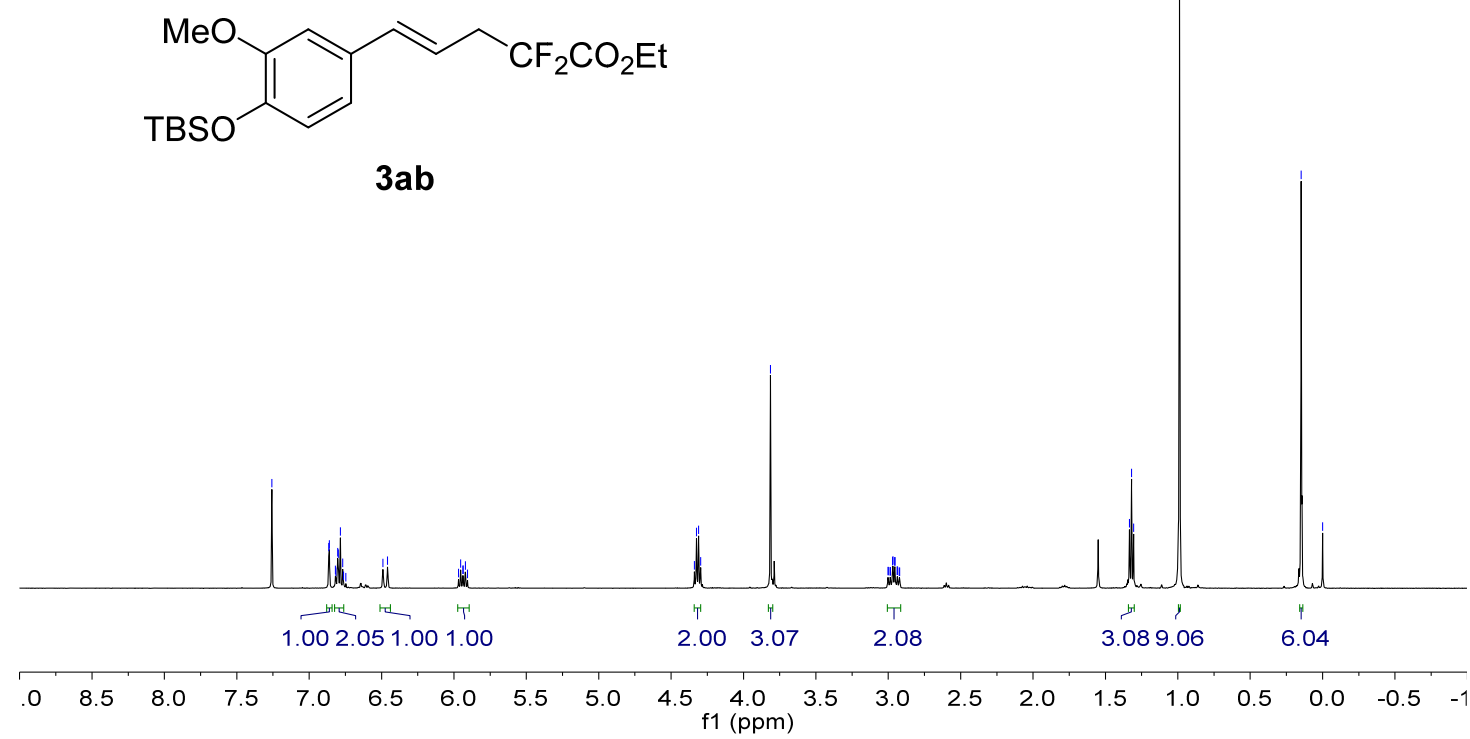

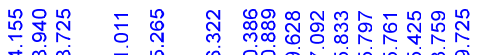

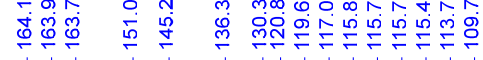

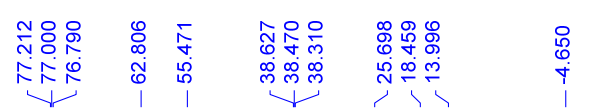

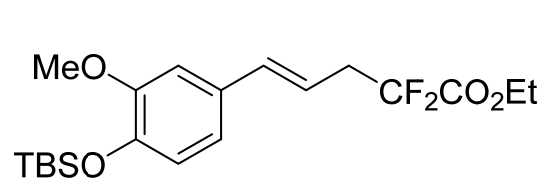

$3 a b$

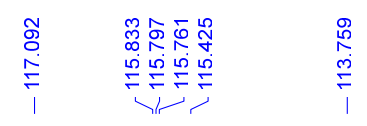

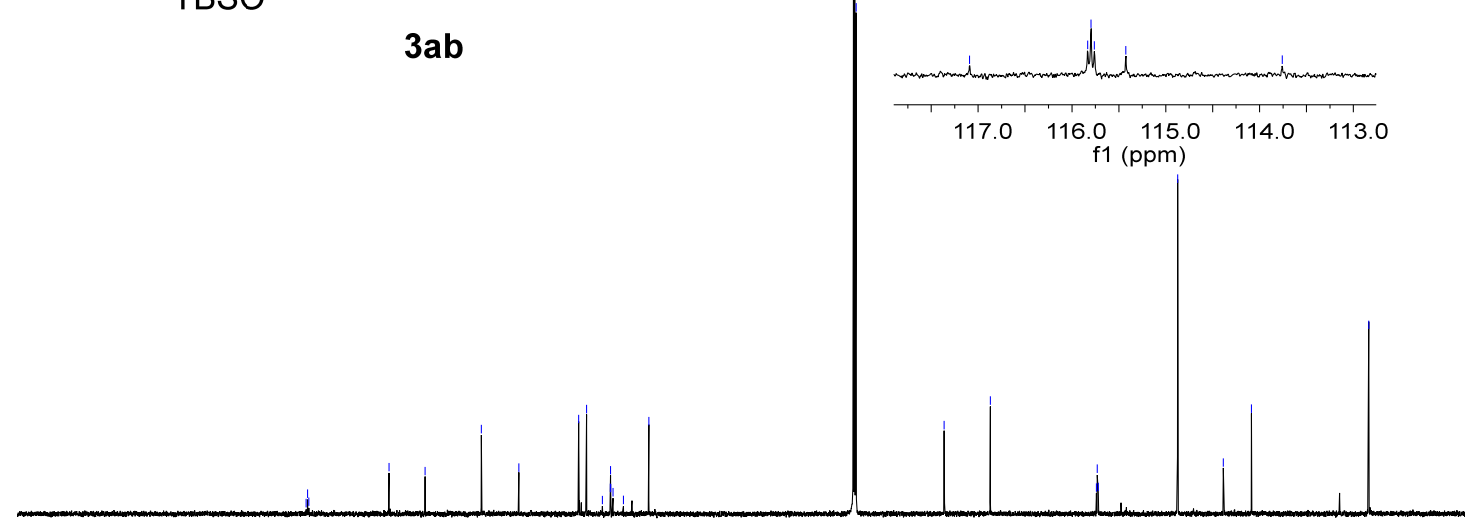

$\begin{array}{llllllllllllllllllllllllll}10 & 200 & 190 & 180 & 170 & 160 & 150 & 140 & 130 & 120 & 110 & \begin{array}{r}100 \\ \mathrm{f} 1(\mathrm{ppm})\end{array} & 80 & 70 & 60 & 50 & 40 & 30 & 20 & 10 & 0 & -10 & -:\end{array}$ 


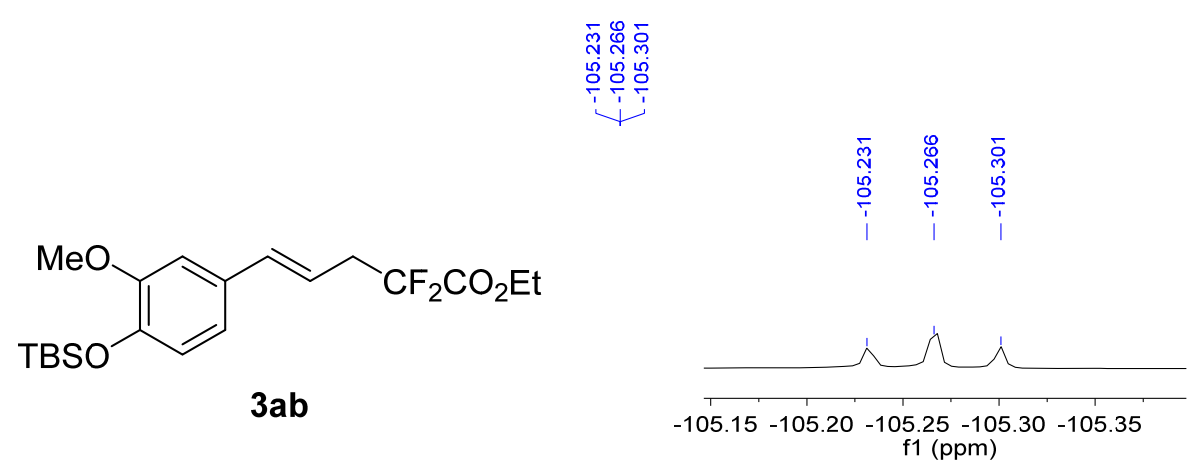

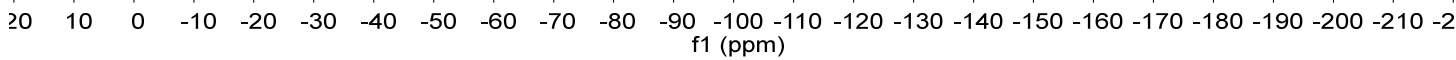

VN<smiles>CCOC(=O)C(F)(F)CC/C=C/c1ccc(Cl)cc1</smiles>

$3 a c$

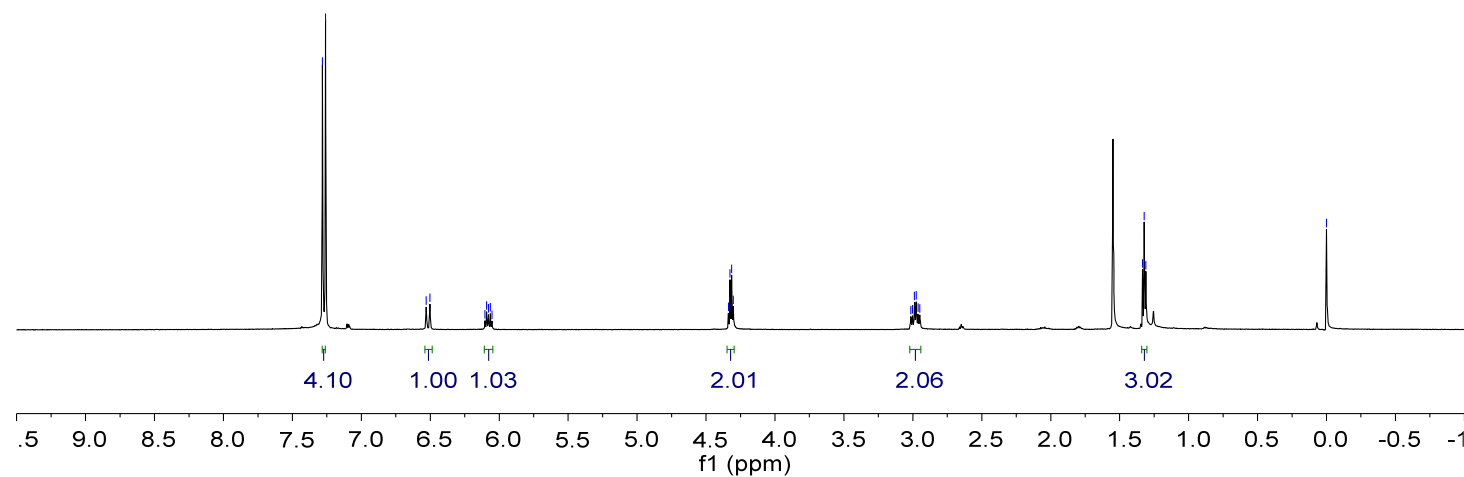




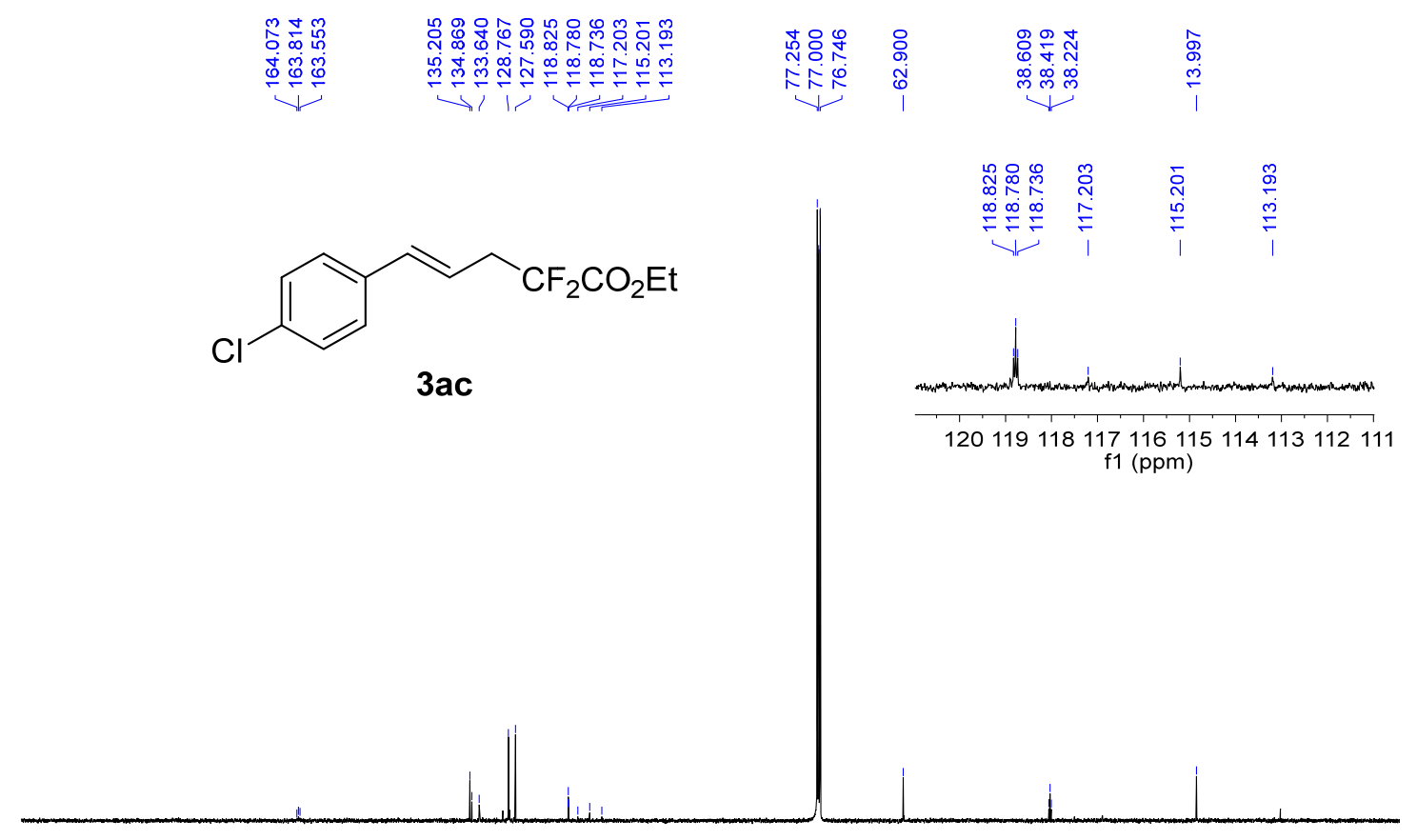

$\begin{array}{lllllllllllllllllllllll}10 & 200 & 190 & 180 & 170 & 160 & 150 & 140 & 130 & 120 & 110 & \begin{array}{l}100 \\ \mathrm{f} 1(\mathrm{ppm})\end{array} & 90 & 70 & 60 & 50 & 40 & 30 & 20 & 10 & 0 & -10 & -4\end{array}$

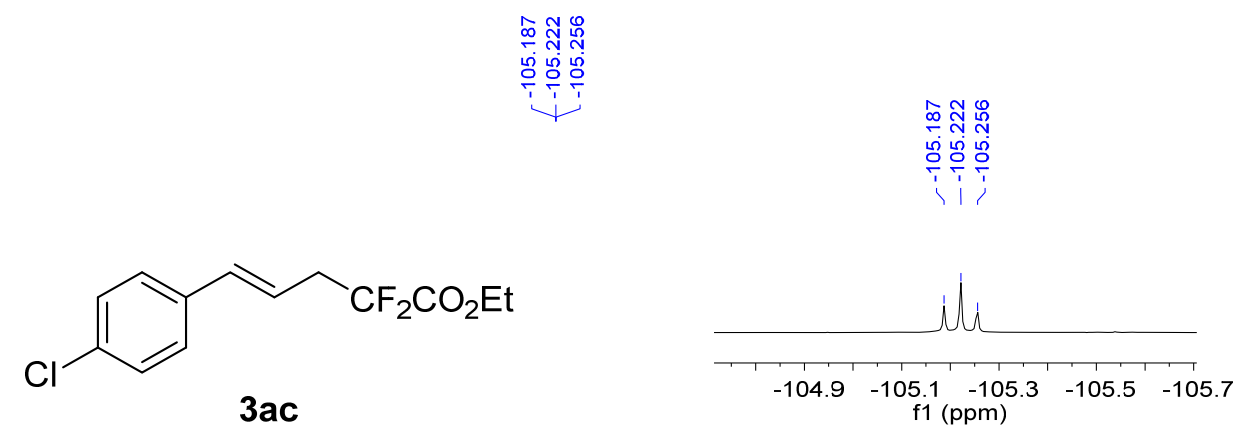

$\begin{array}{lllllllllllllllllllllll}0 & -10 & -20 & -30 & -40 & -50 & -60 & -70 & -80 & -90 & -100 & -110 & -120 & -130 & -140 & -150 & -160 & -170 & -180 & -190 & -200 & -210\end{array}$ 
<smiles>CCOC(=O)C(F)(F)CC=Cc1ccc(-c2ccccc2)cc1</smiles>

3ad

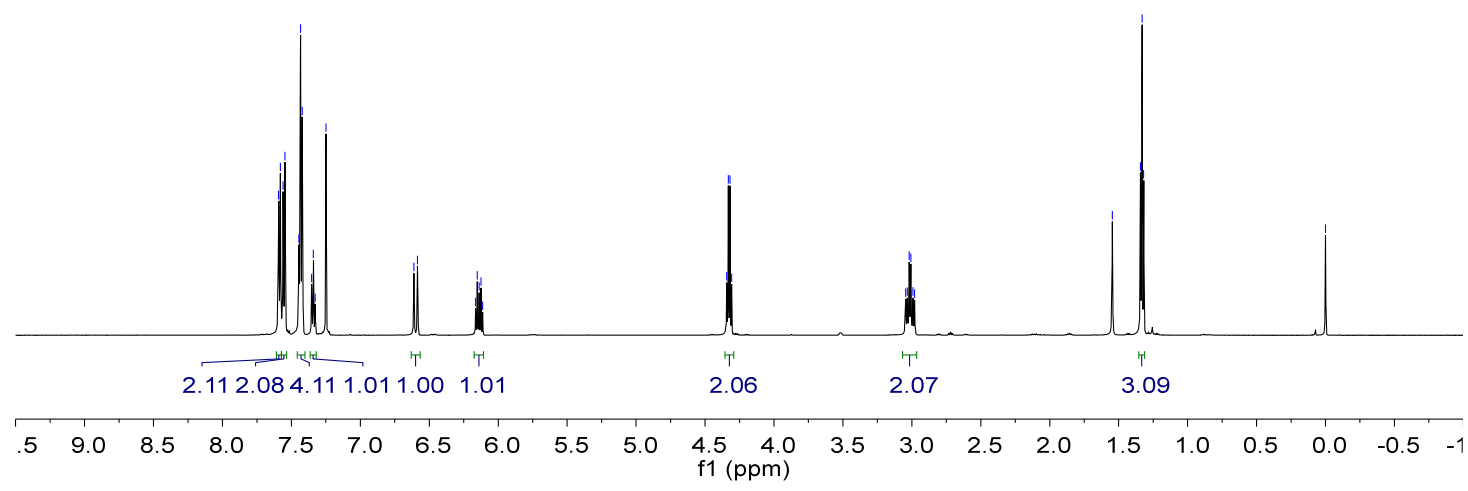

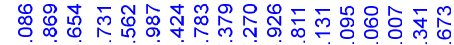

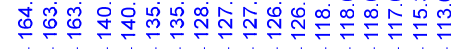

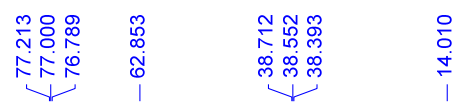<smiles>CCOC(=O)CCC=Cc1ccc(-c2ccccc2)cc1</smiles>

3ad

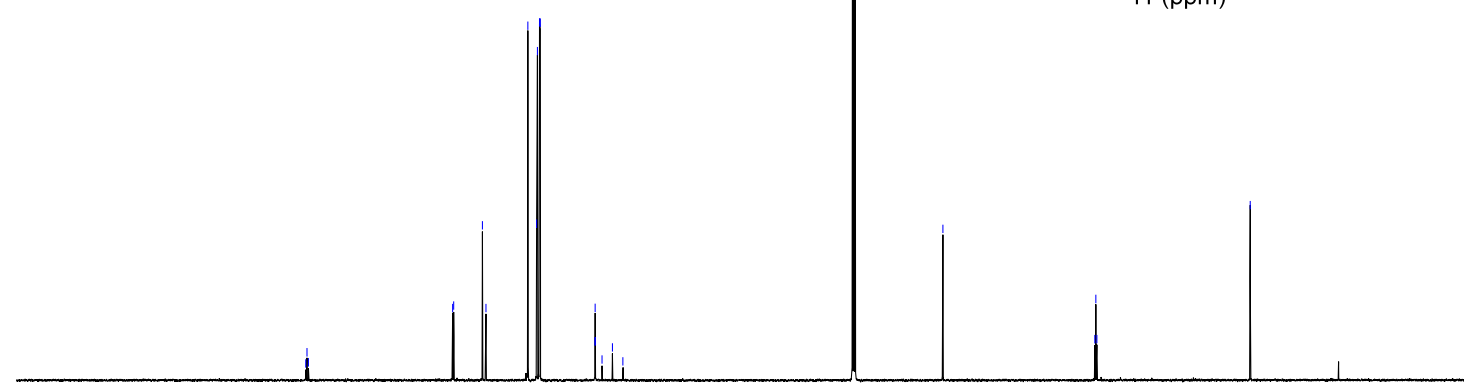

$\begin{array}{lllllllllllllllllllllll}10 & 200 & 190 & 180 & 170 & 160 & 150 & 140 & 130 & 120 & 110 & \begin{array}{r}100 \\ \mathrm{f} 1(\mathrm{ppm})\end{array} & 80 & 70 & 60 & 50 & 40 & 30 & 20 & 10 & 0 & -10 & -:\end{array}$ 

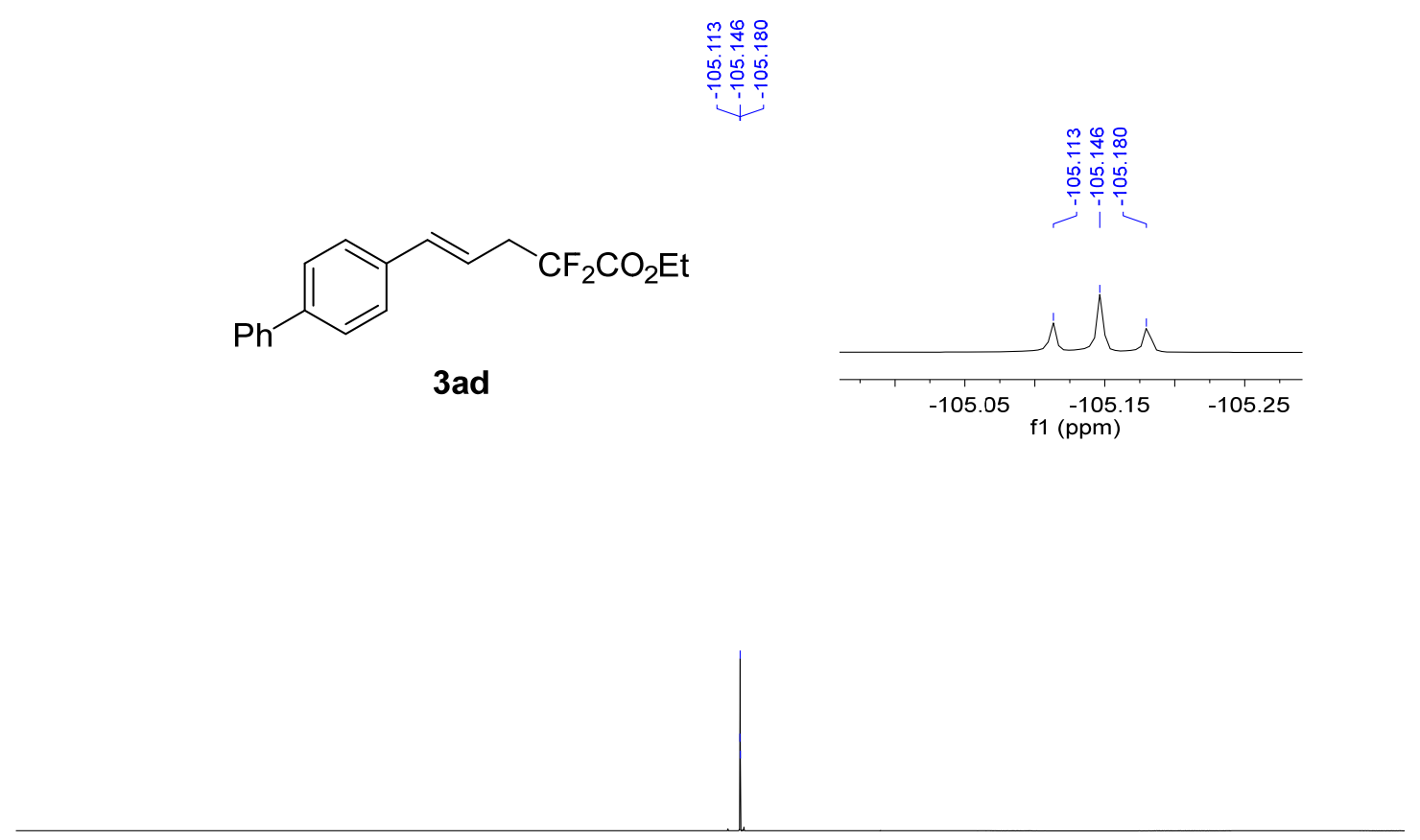

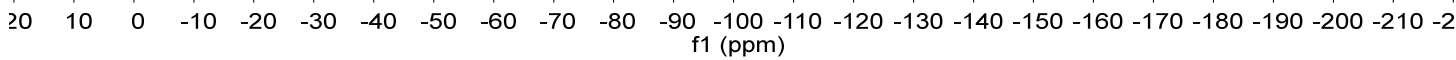

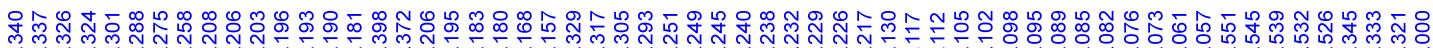

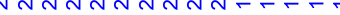

$\mathrm{Ph} \curvearrowright \mathrm{H}_{3}-\mathrm{CF}_{2} \mathrm{CO}_{2} \mathrm{Et}$

3ae

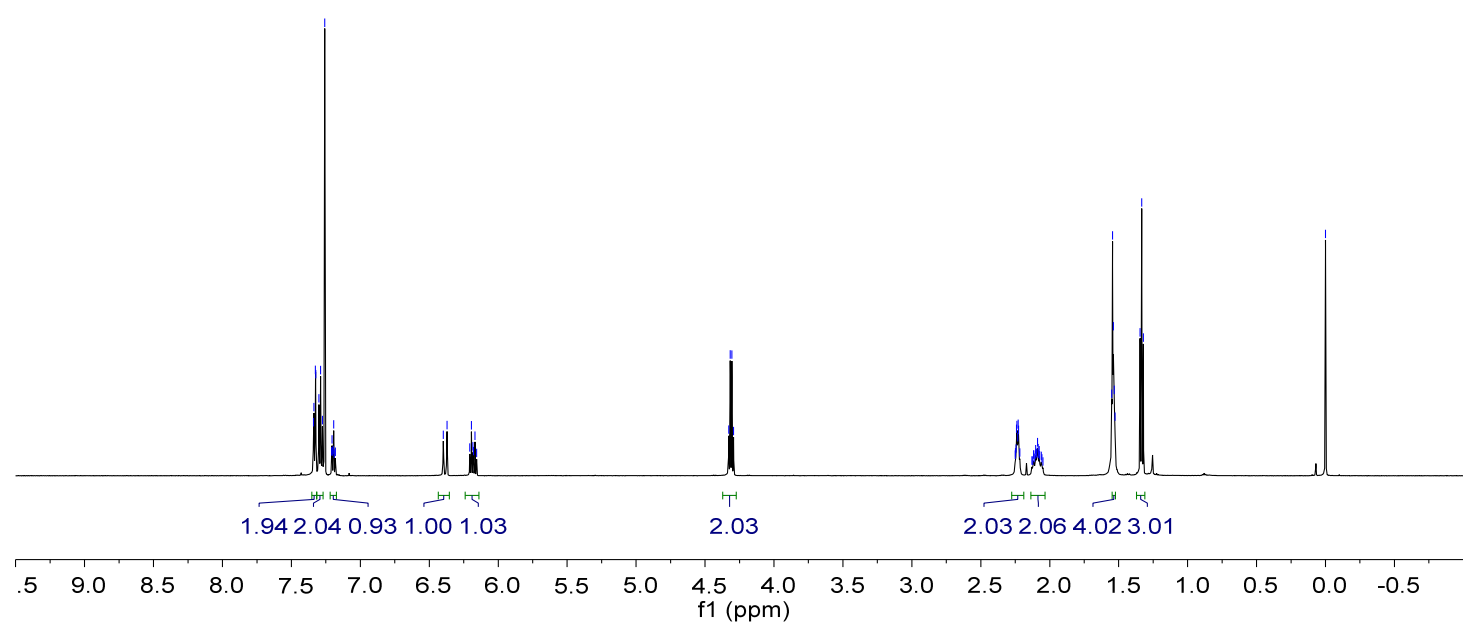




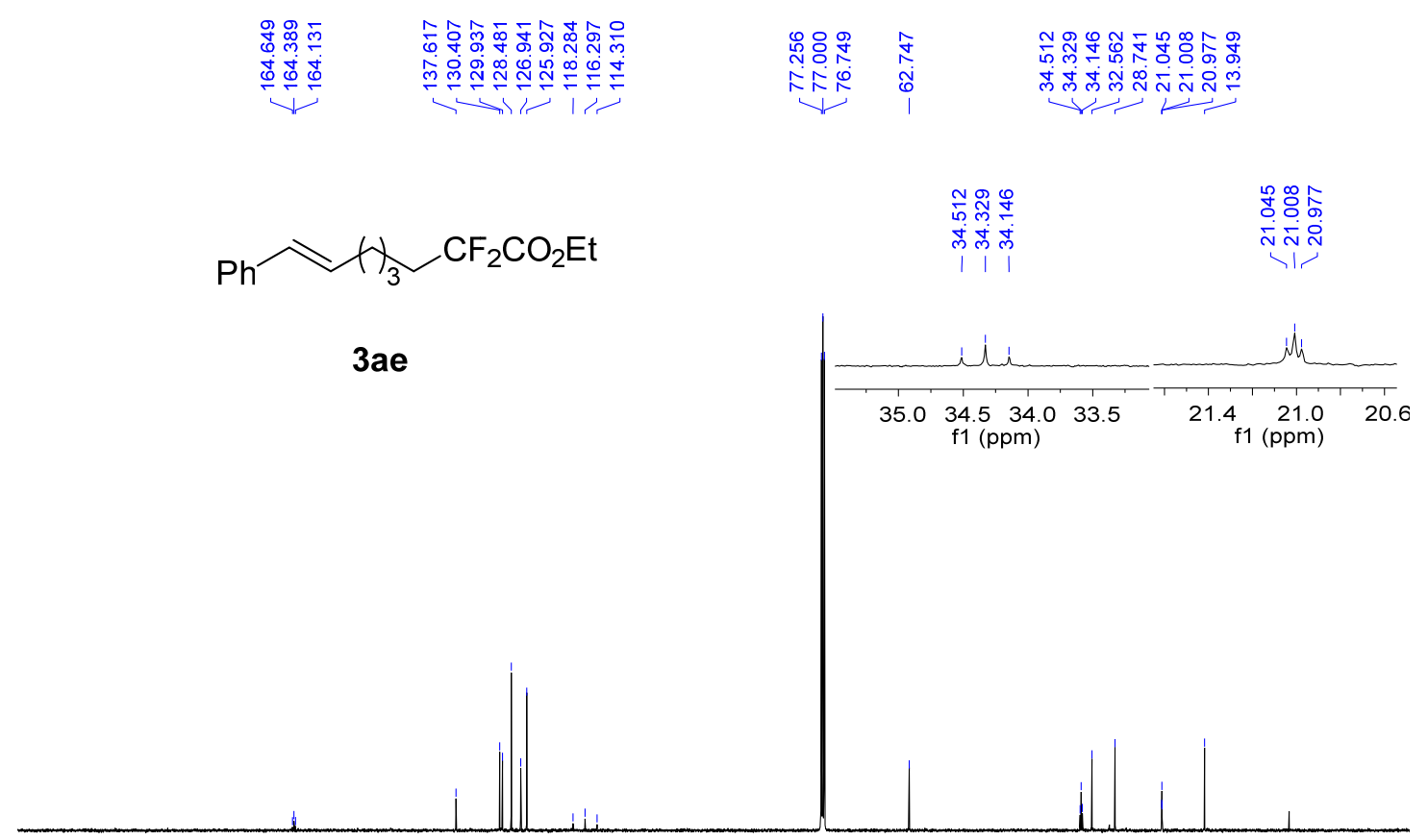

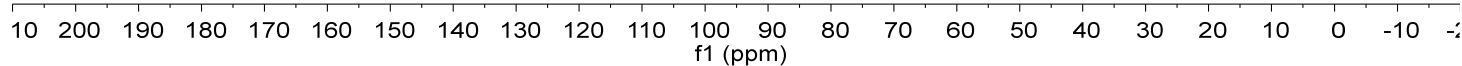

识品品

ㅇํㅇ

$+1$

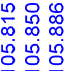

$\mathrm{Ph} \sim \mathrm{H}_{3} \sim \mathrm{CF}_{2} \mathrm{CO}_{2} \mathrm{Et}$

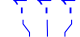

$3 a e$

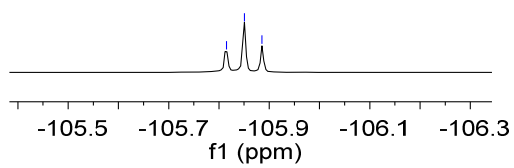

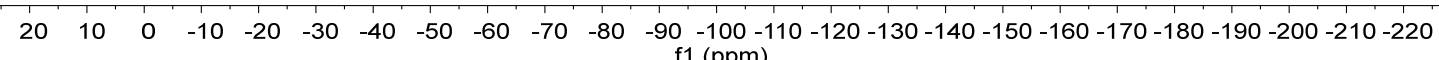



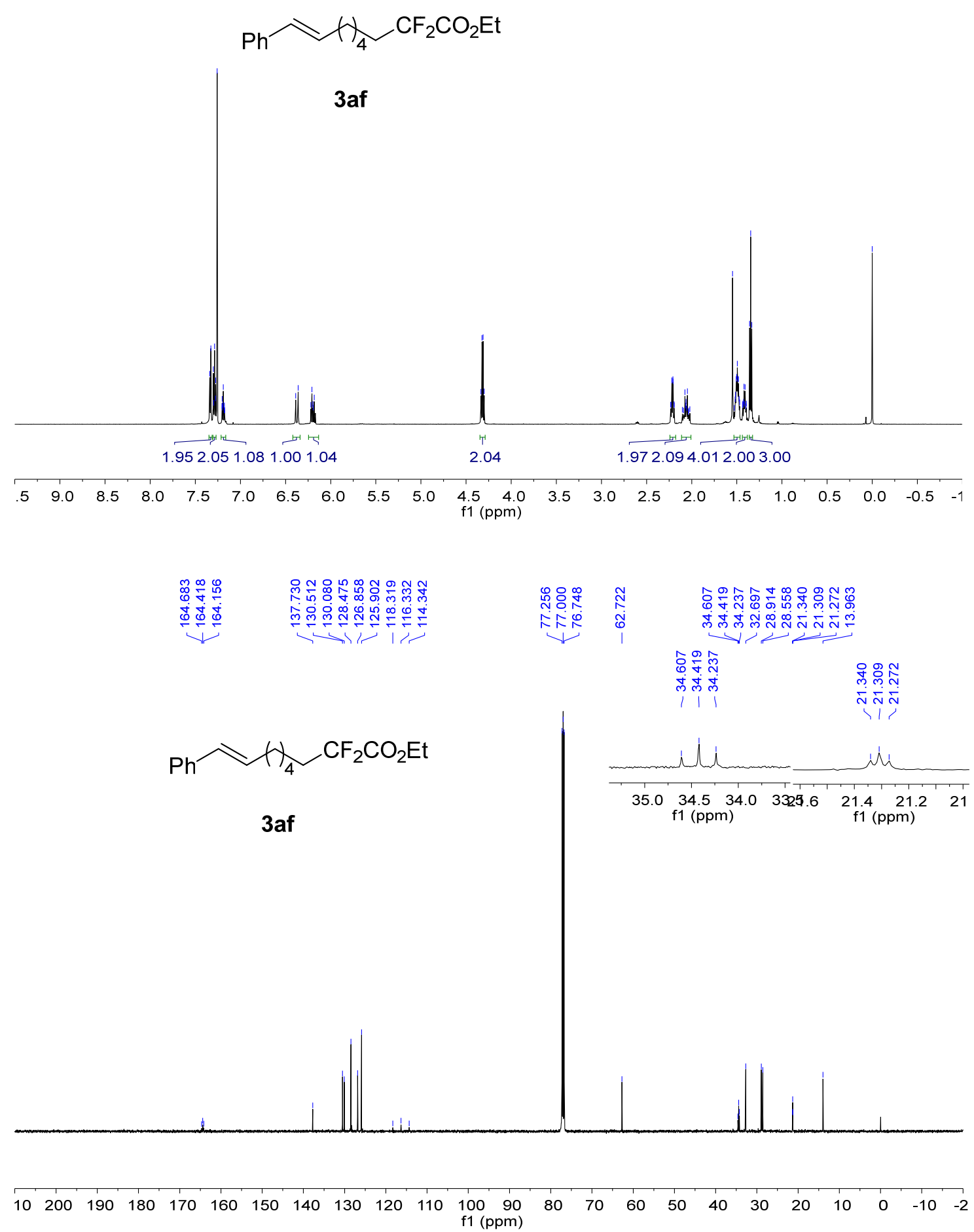
$\mathrm{Ph} \curvearrowright \mathrm{H}_{4}-\mathrm{CF}_{2} \mathrm{CO}_{2} \mathrm{Et}$

3af

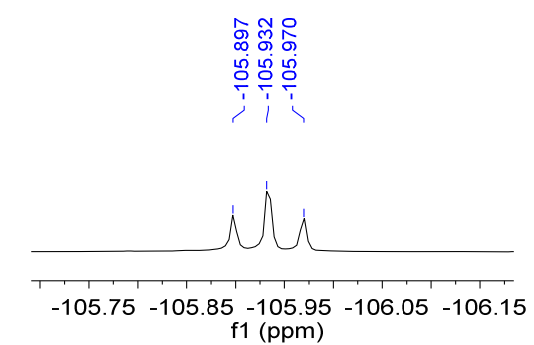

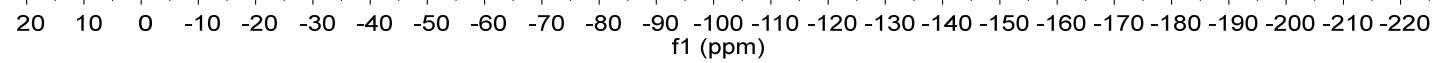

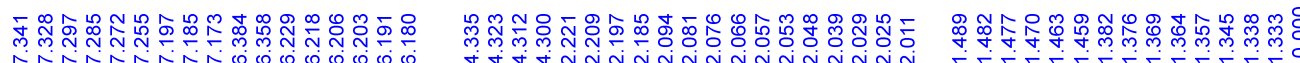

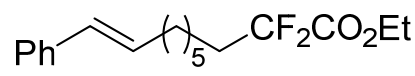

\section{3ag}

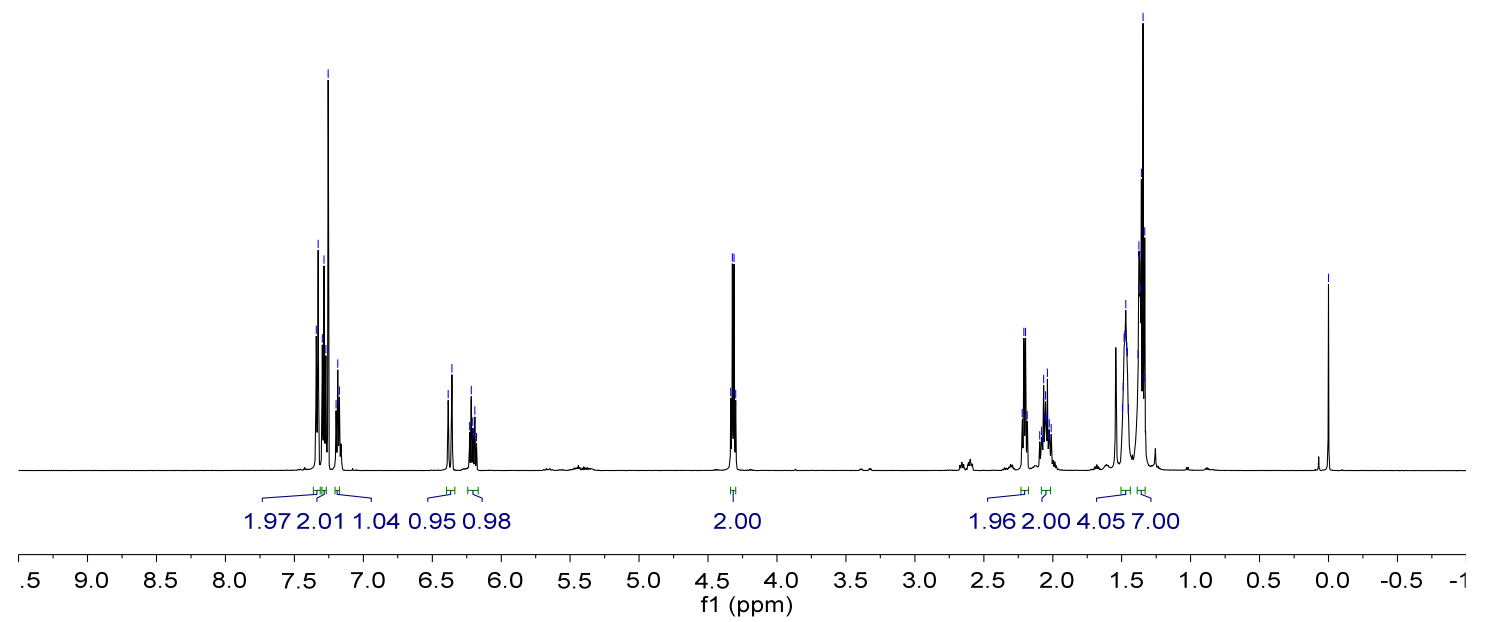




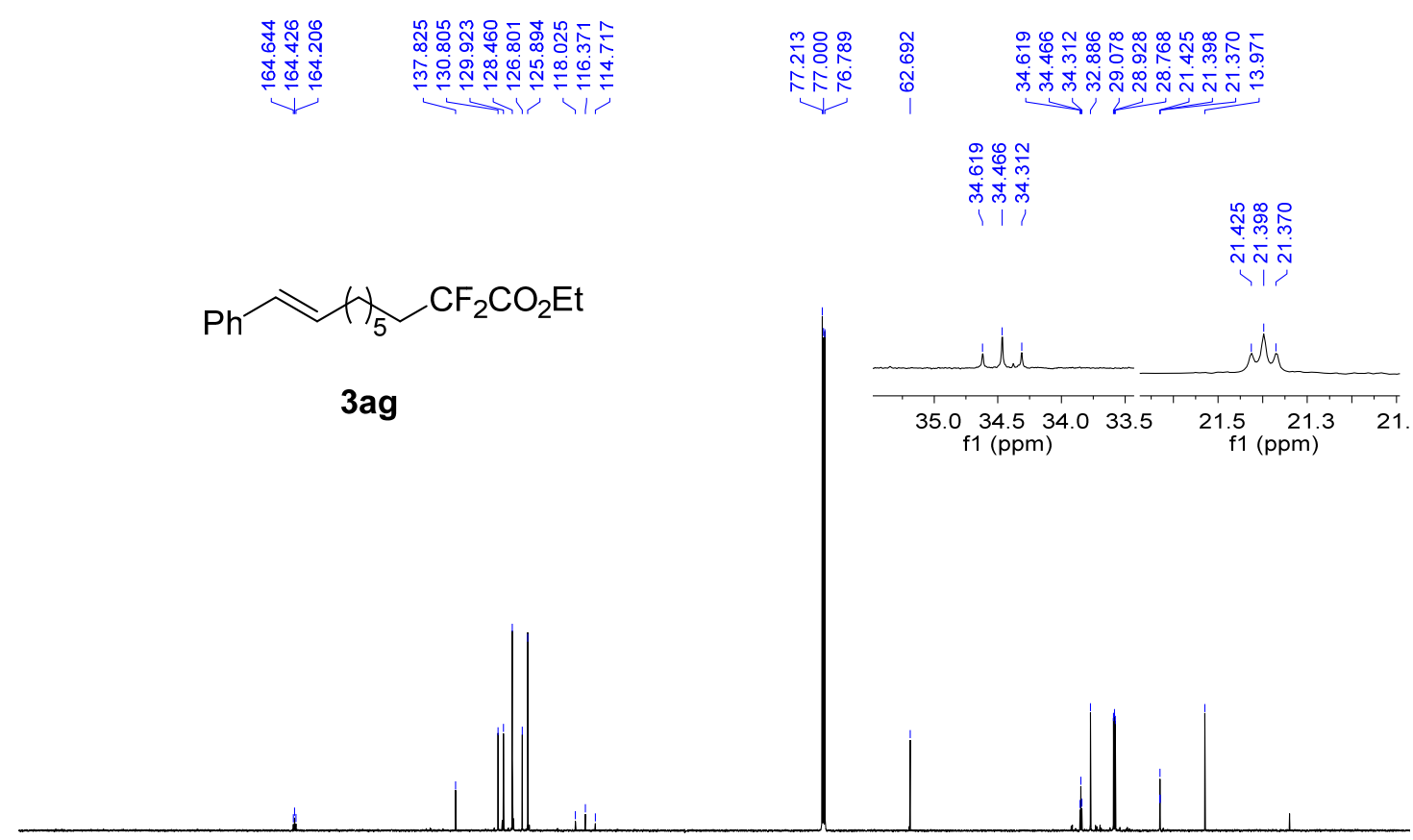

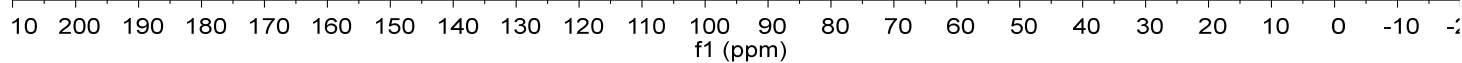
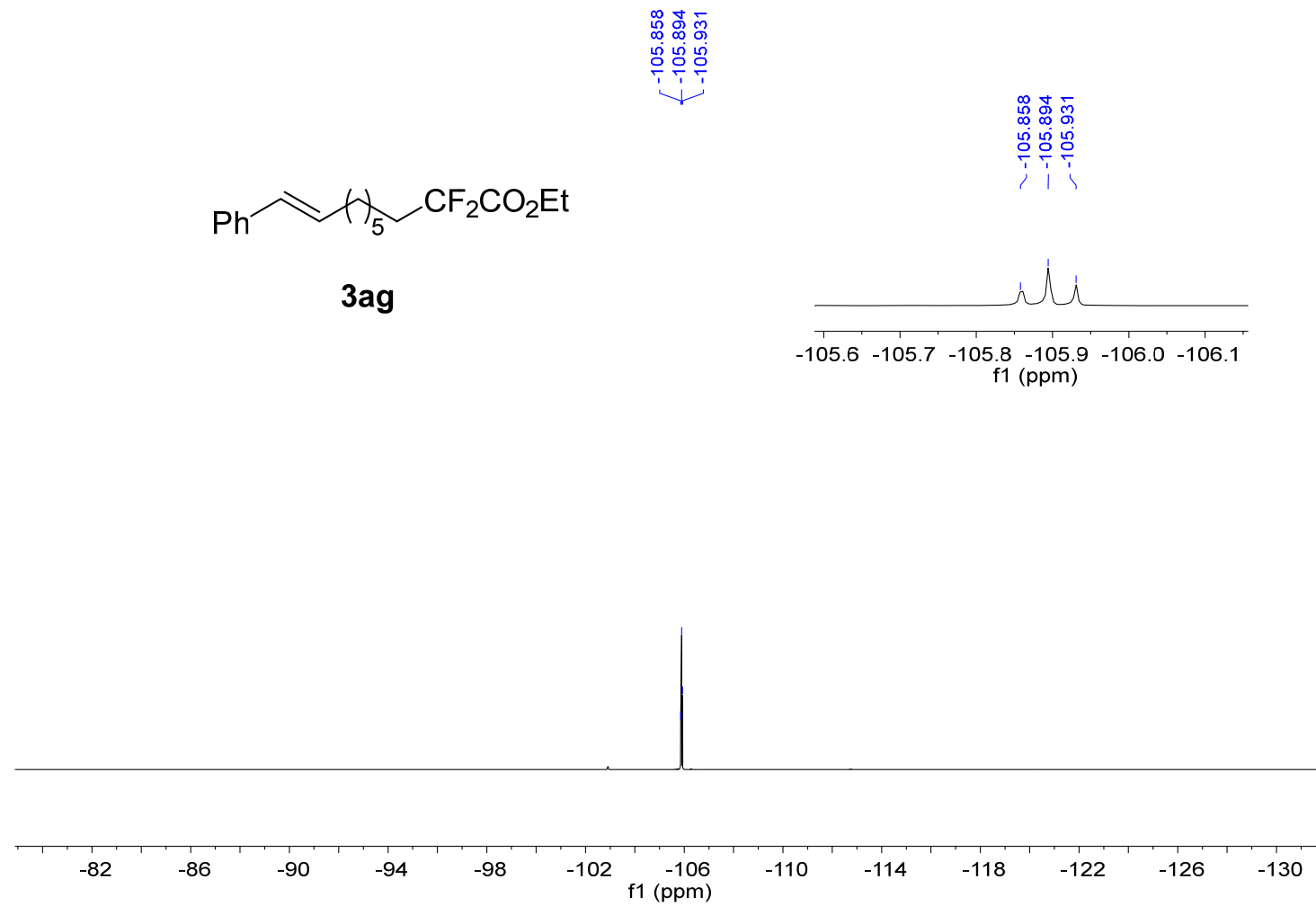


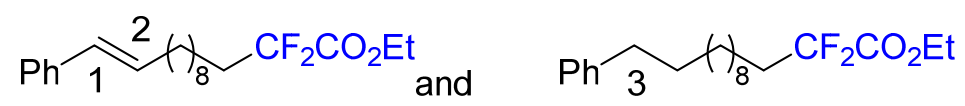

3ah

3ah'

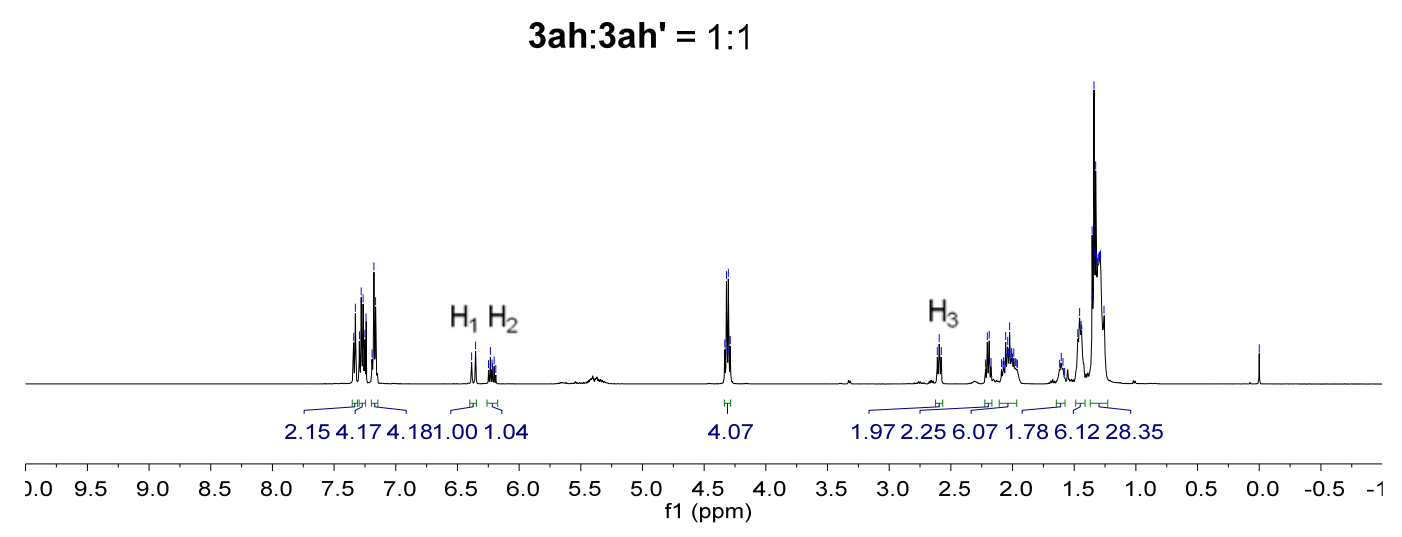

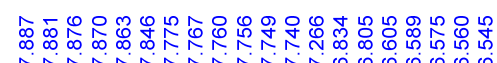

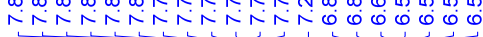

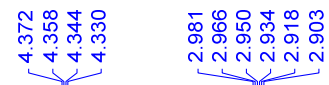

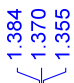

8
0
0
1

PhthN $\mathrm{CF}_{2} \mathrm{CO}_{2} \mathrm{Et}$

3ai

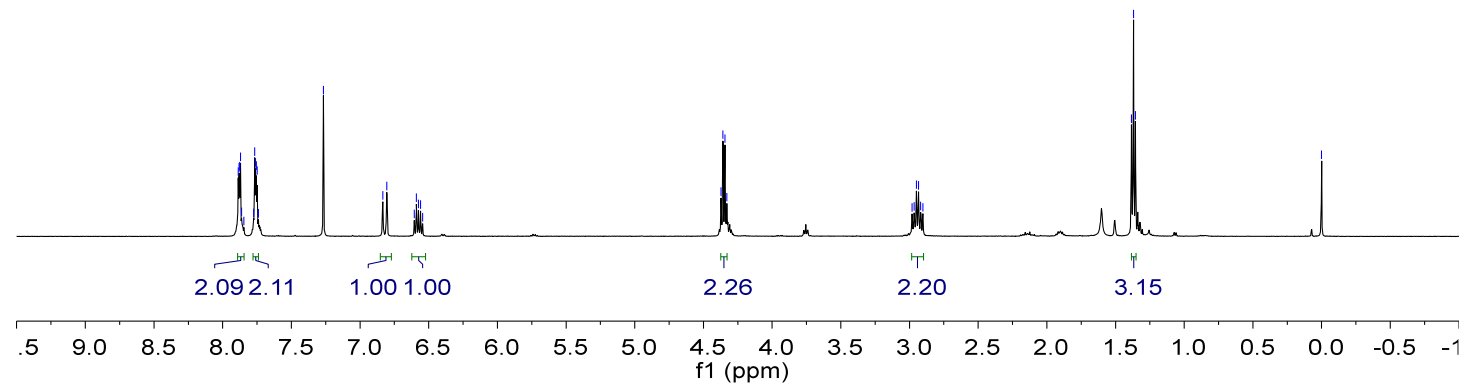


$\mathrm{PhthN} \leadsto \mathrm{CF}_{2} \mathrm{CO}_{2} \mathrm{Et}$

3ai

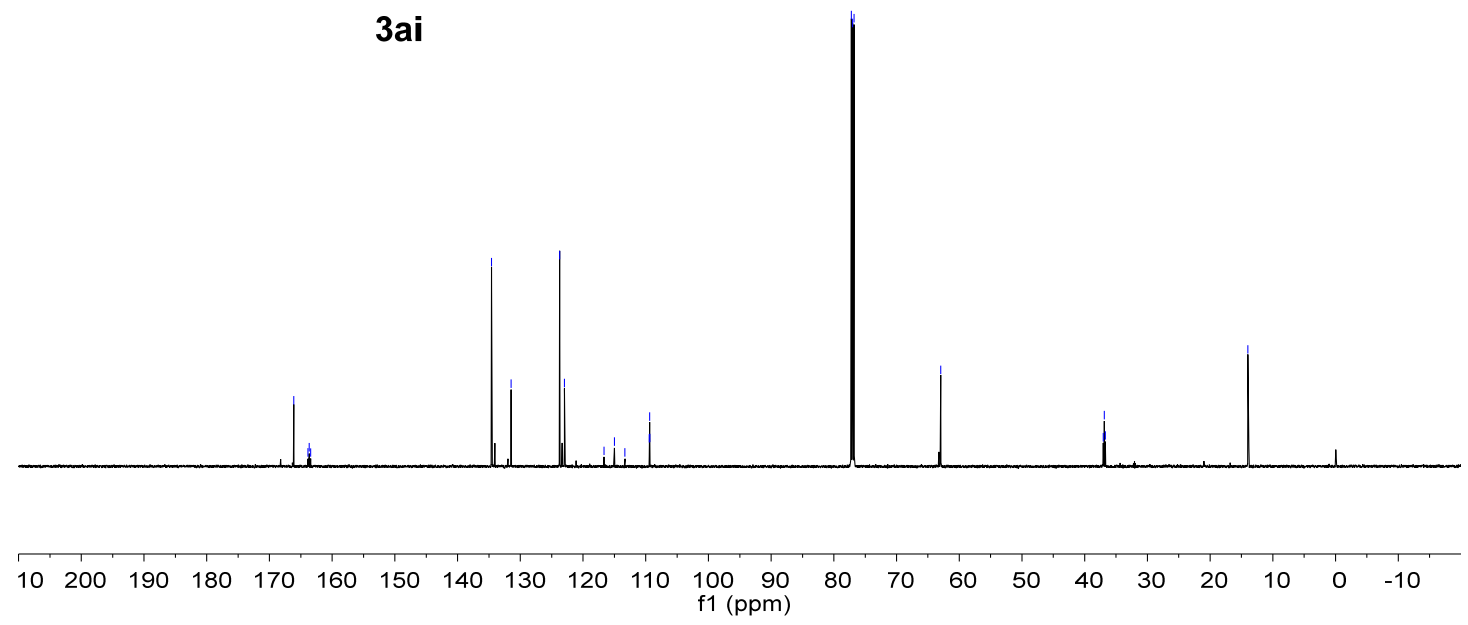

获

웅 웅

PhthN $\mathrm{CF}_{2} \mathrm{CO}_{2} \mathrm{Et}$

3ai

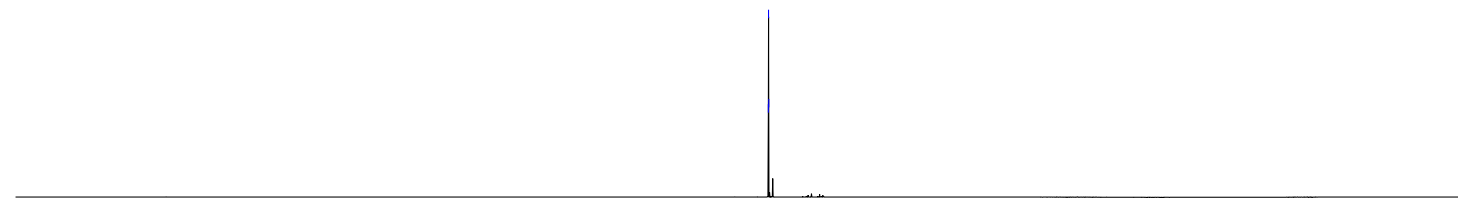

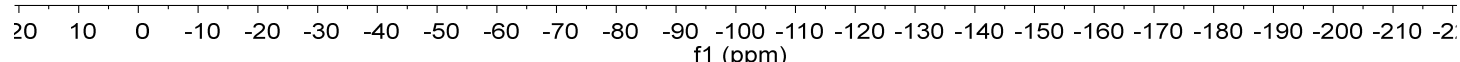


$\mathrm{Ph}^{-\mathrm{N}} \prod_{\mathrm{O}}^{\mathrm{C}} \sim \mathrm{CF}_{2} \mathrm{CO}_{2} \mathrm{Et}$

3aj
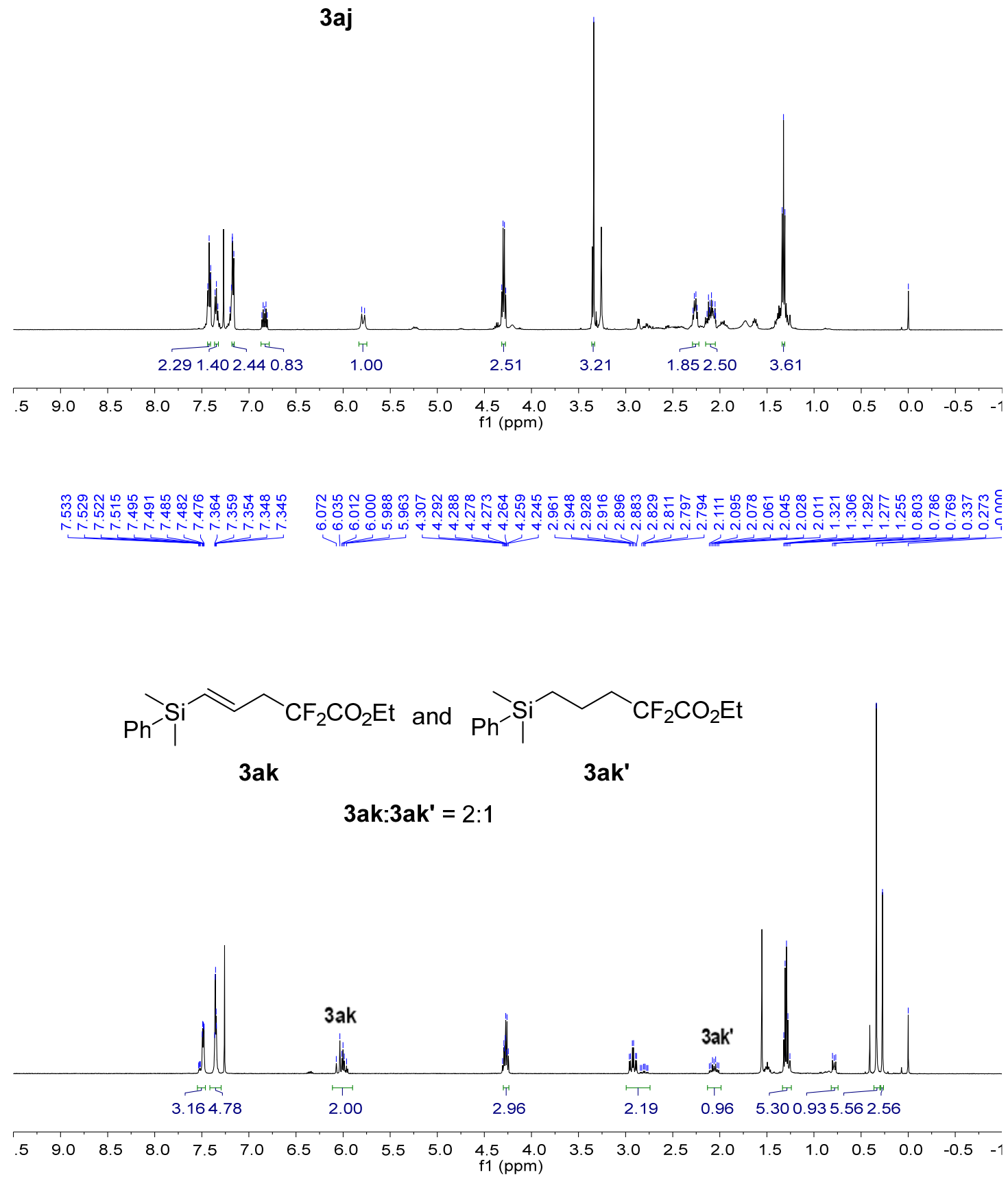
<smiles>COC(=O)C(F)(F)CC/C=C/c1ccccc1</smiles>

3al

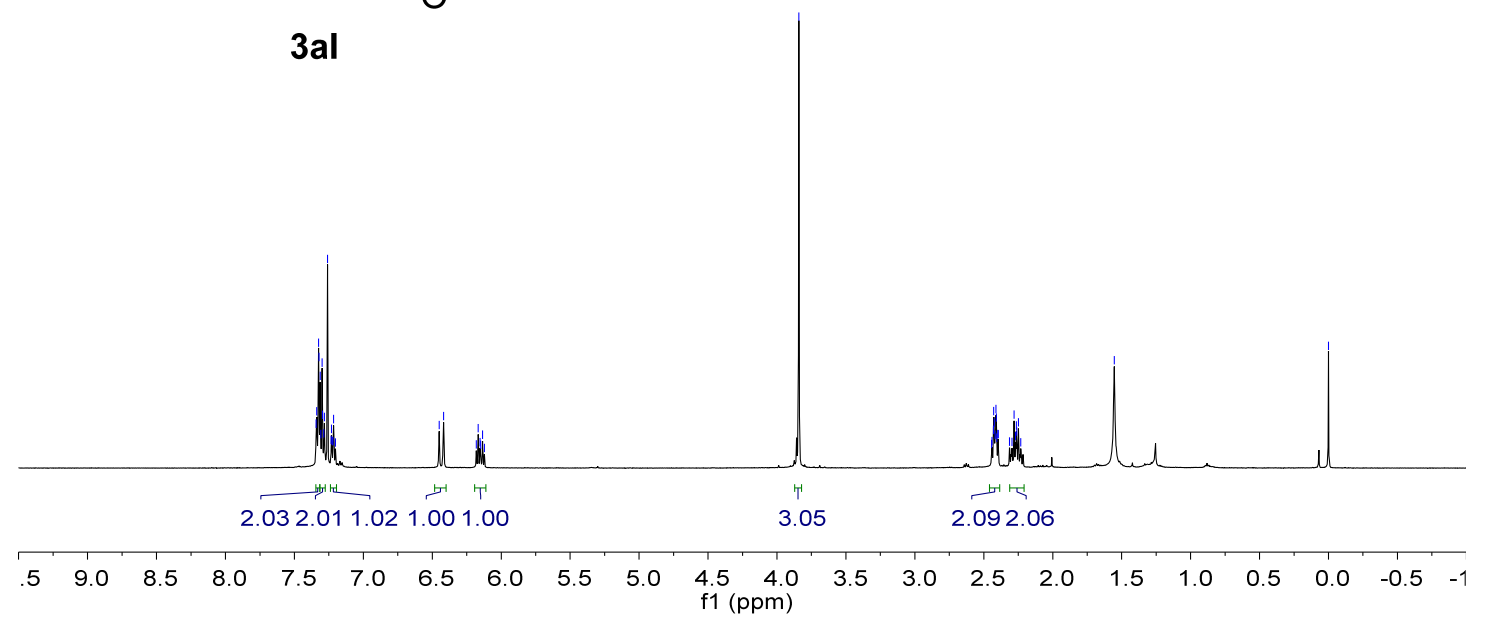

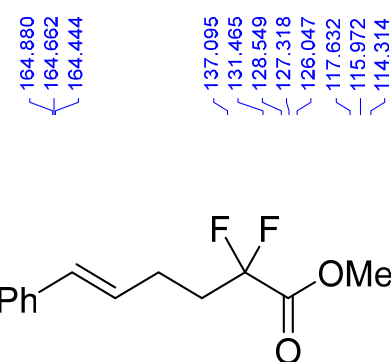

3al

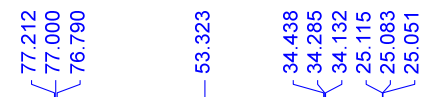

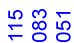

ผ

广于

\I?

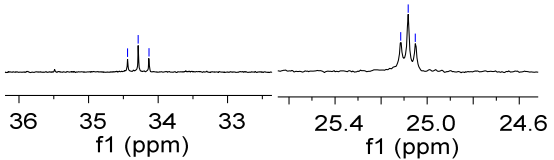

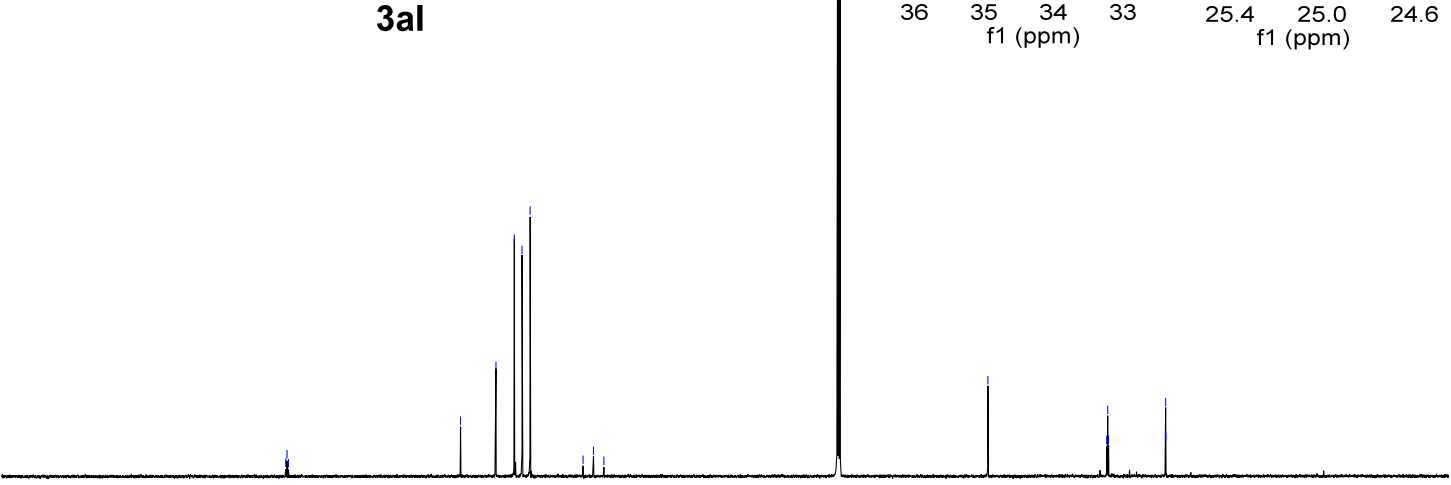

$\begin{array}{llllllllllllllllllllllll}10 & 200 & 190 & 180 & 170 & 160 & 150 & 140 & 130 & 120 & 110 & \begin{array}{l}100 \\ \mathrm{f} 1(\mathrm{ppm})\end{array} & 80 & 70 & 60 & 50 & 40 & 30 & 20 & 10 & 0 & -10 & -2\end{array}$ 

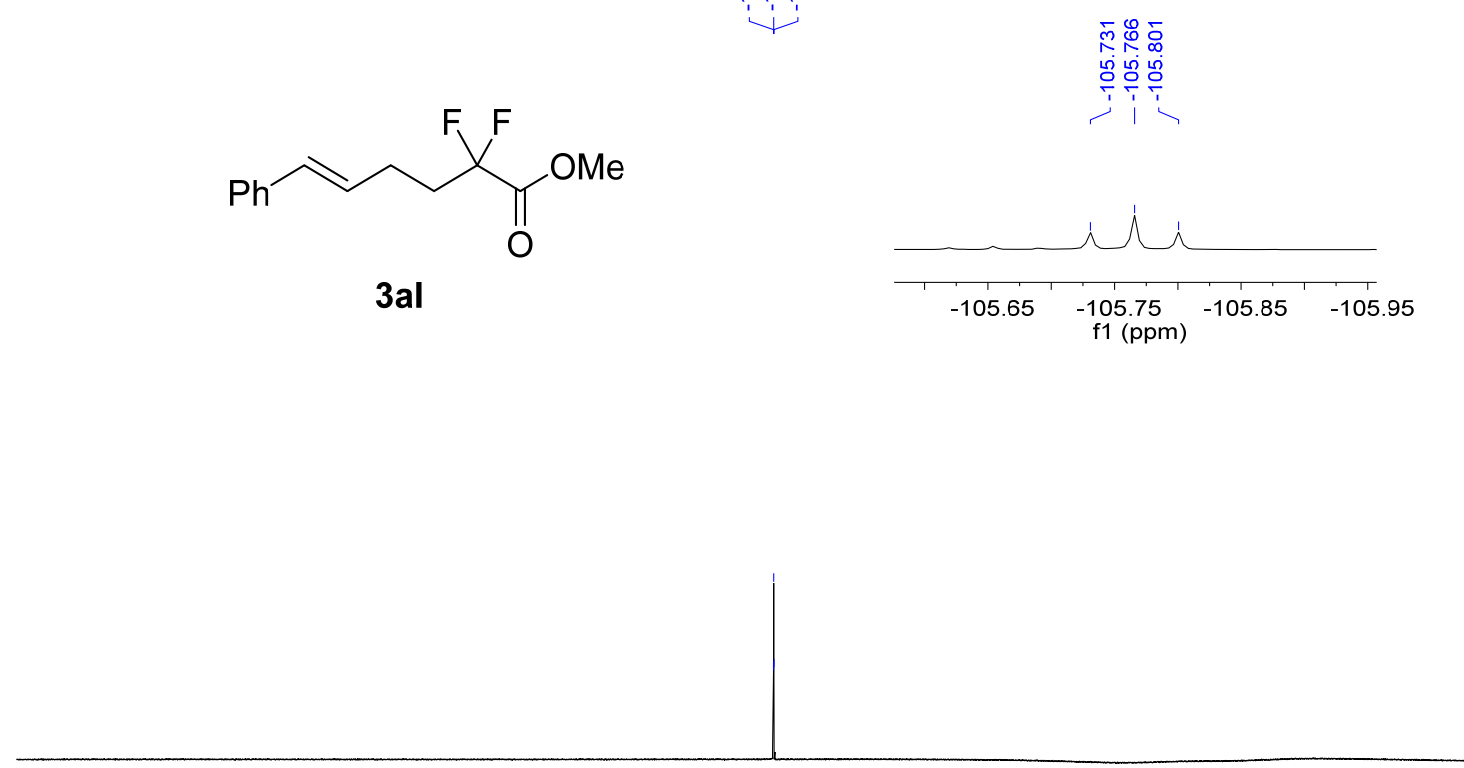

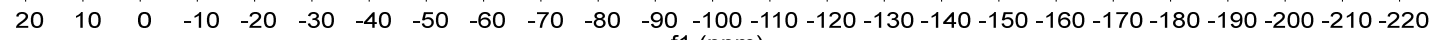
f1 $(\mathrm{ppm})$<smiles>O=C(OCc1ccccc1)C(F)(F)CC/C=C/c1ccccc1</smiles>

3am

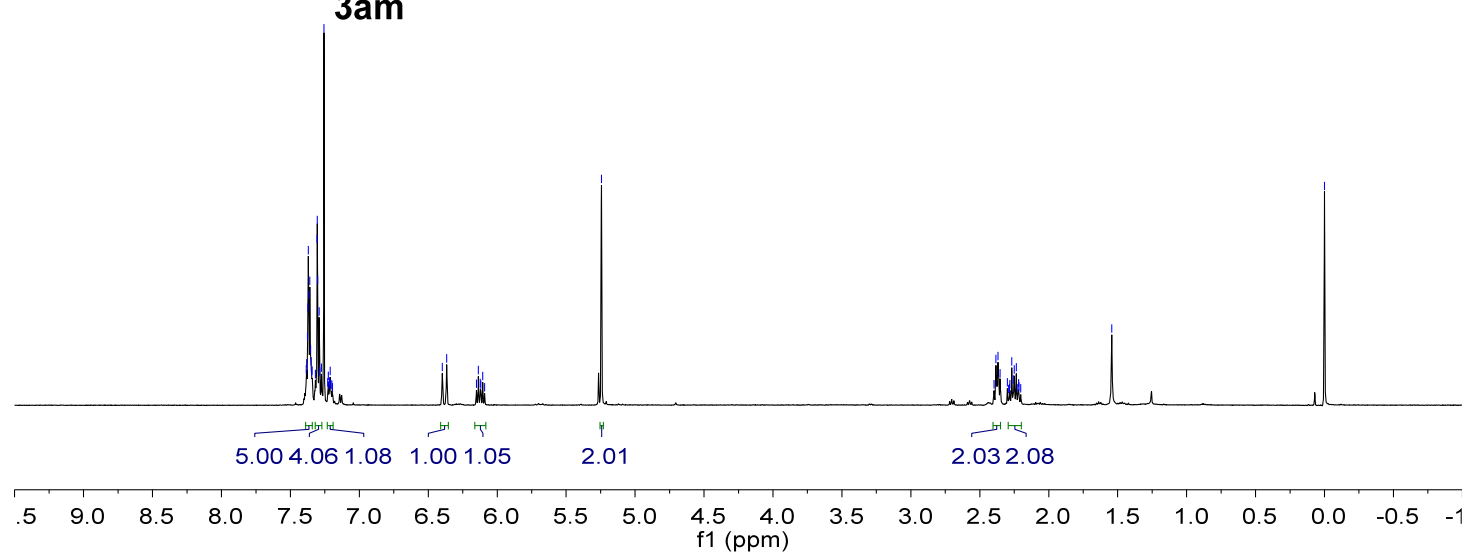




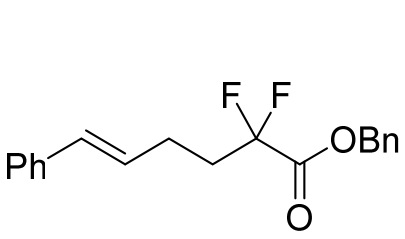

3am
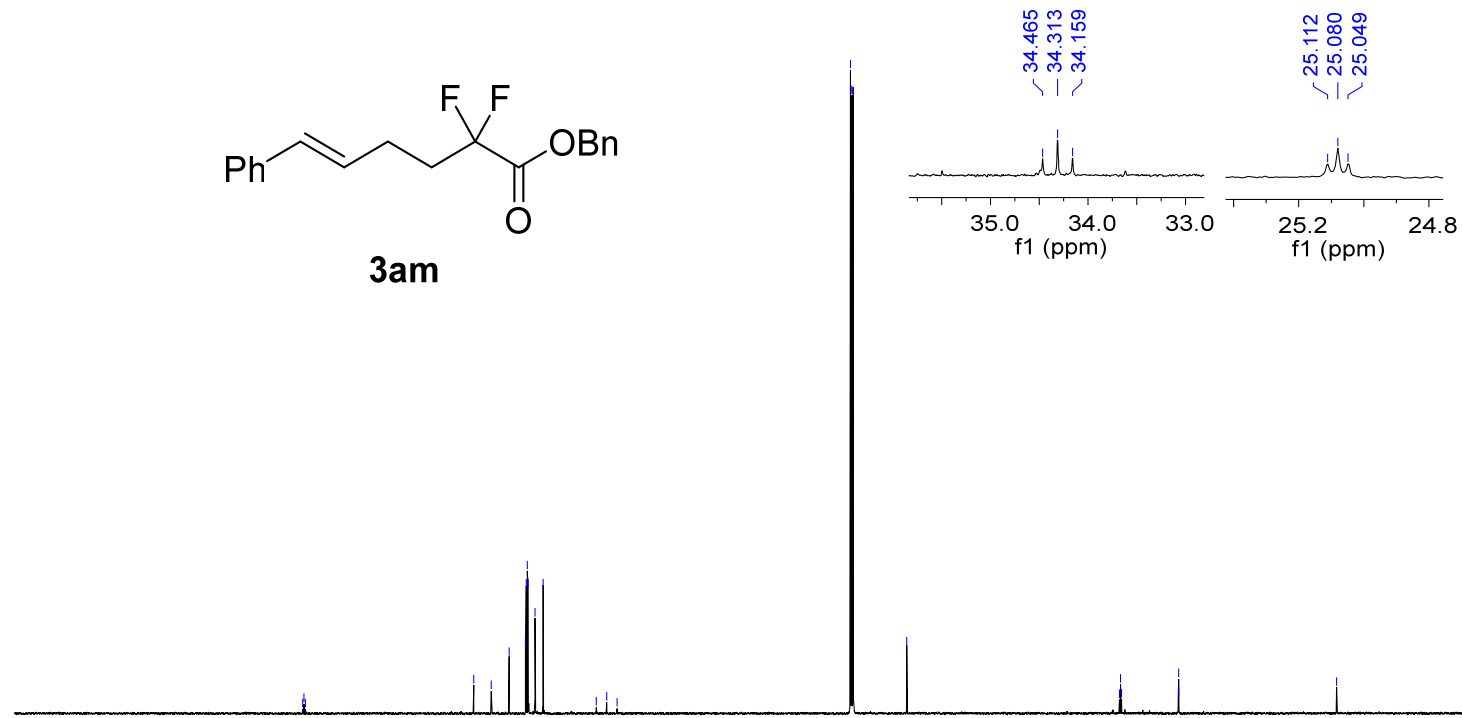

$\begin{array}{rlrllllllllllllllllllll}10 & 200 & 190 & 180 & 170 & 160 & 150 & 140 & 130 & 120 & 110 & \begin{array}{l}100 \\ \mathrm{f} 1\end{array}(\mathrm{ppm}) & 80 & 70 & 60 & 50 & 40 & 30 & 20 & 10 & 0 & -10 & -4\end{array}$

$\sqrt{10}$

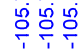<smiles>O=C(OCc1ccccc1)C(F)(F)CC/C=C/c1ccccc1</smiles>

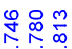

兽融总

3am

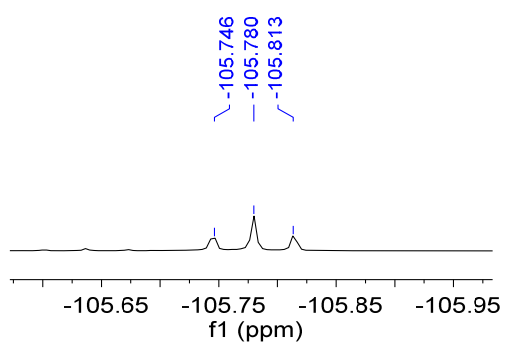

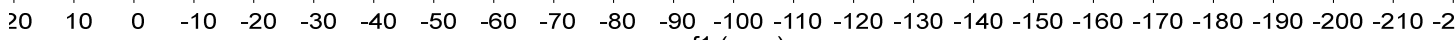
f1 (ppm) 
<smiles>CCCc1ccc(COC(=O)C(F)(F)CC/C=C/c2ccccc2)cc1</smiles>

3an

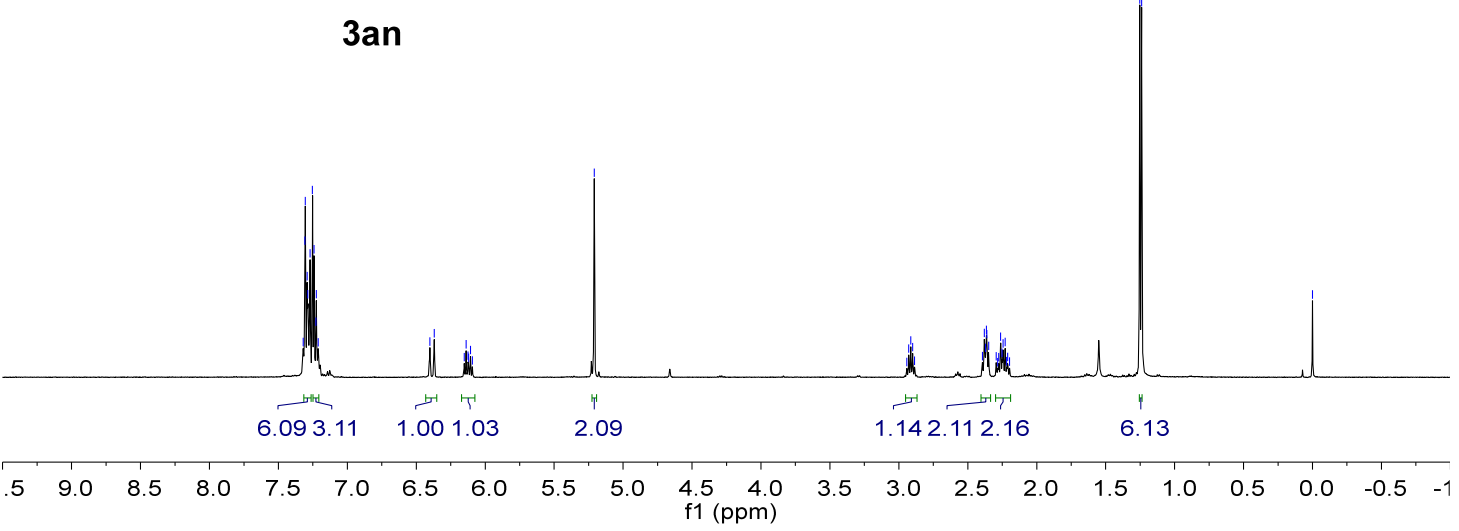

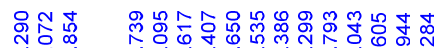

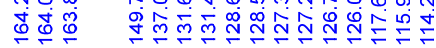

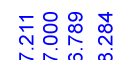

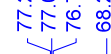

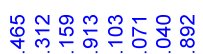

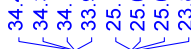

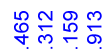

लें लें ले

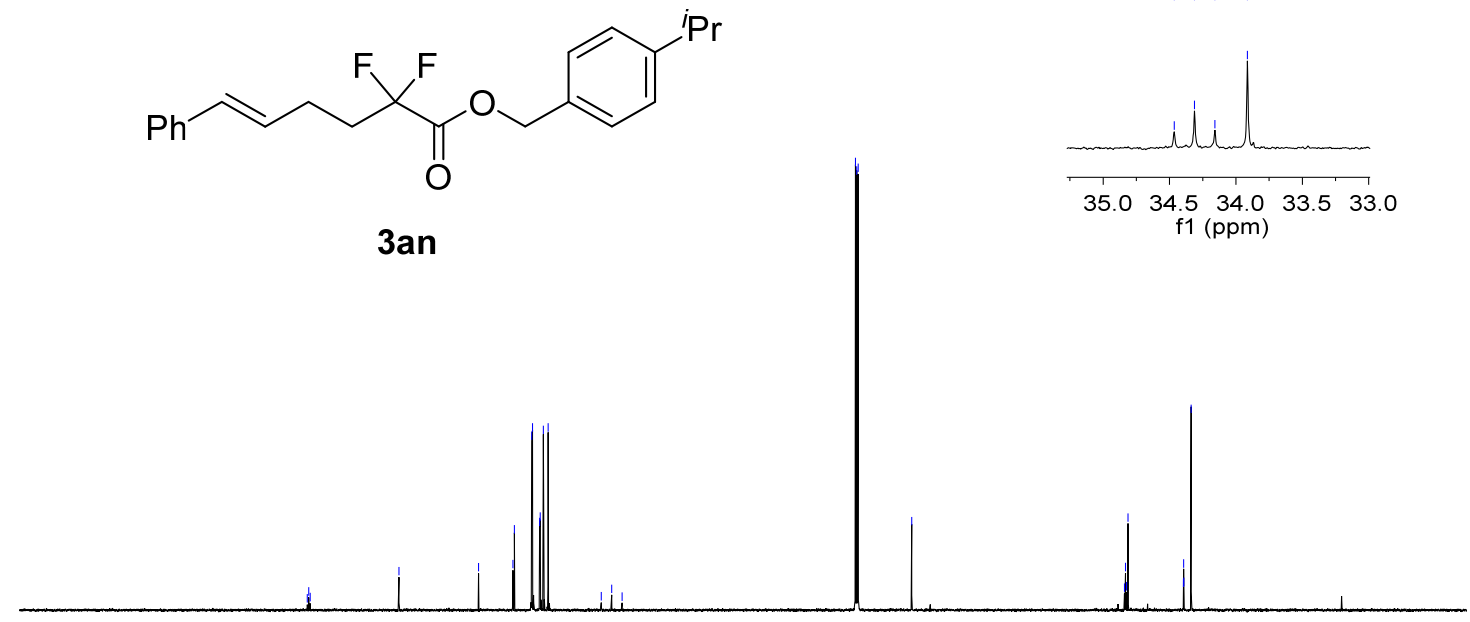

| । ।

$\begin{array}{lllllllllllllllllllllllll}10 & 200 & 190 & 180 & 170 & 160 & 150 & 140 & 130 & 120 & 110 & \begin{array}{r}100 \\ \mathrm{f} 1(\mathrm{ppm})\end{array} & 80 & 70 & 60 & 50 & 40 & 30 & 20 & 10 & 0 & -10 & -:\end{array}$ 

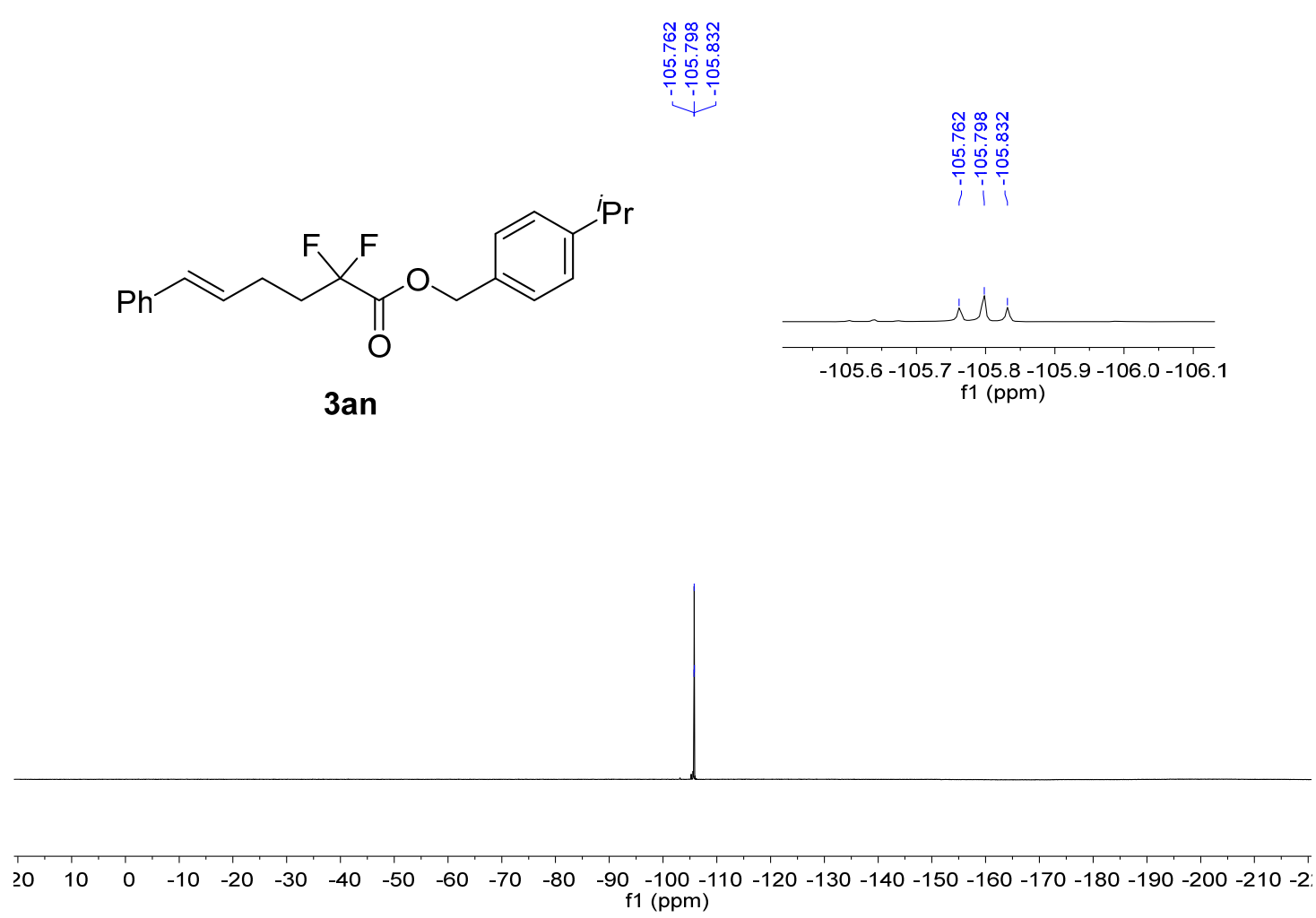<smiles>O=C(OCc1ccc(F)cc1)C(F)(F)CC/C=C/c1ccccc1</smiles>

3 ao

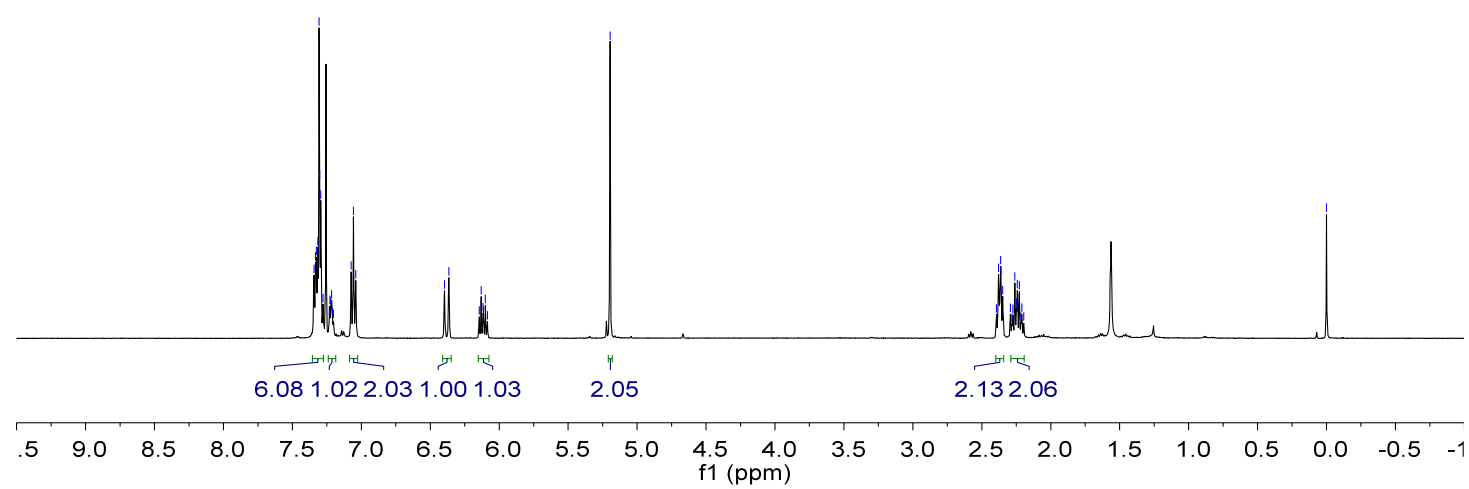




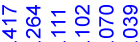
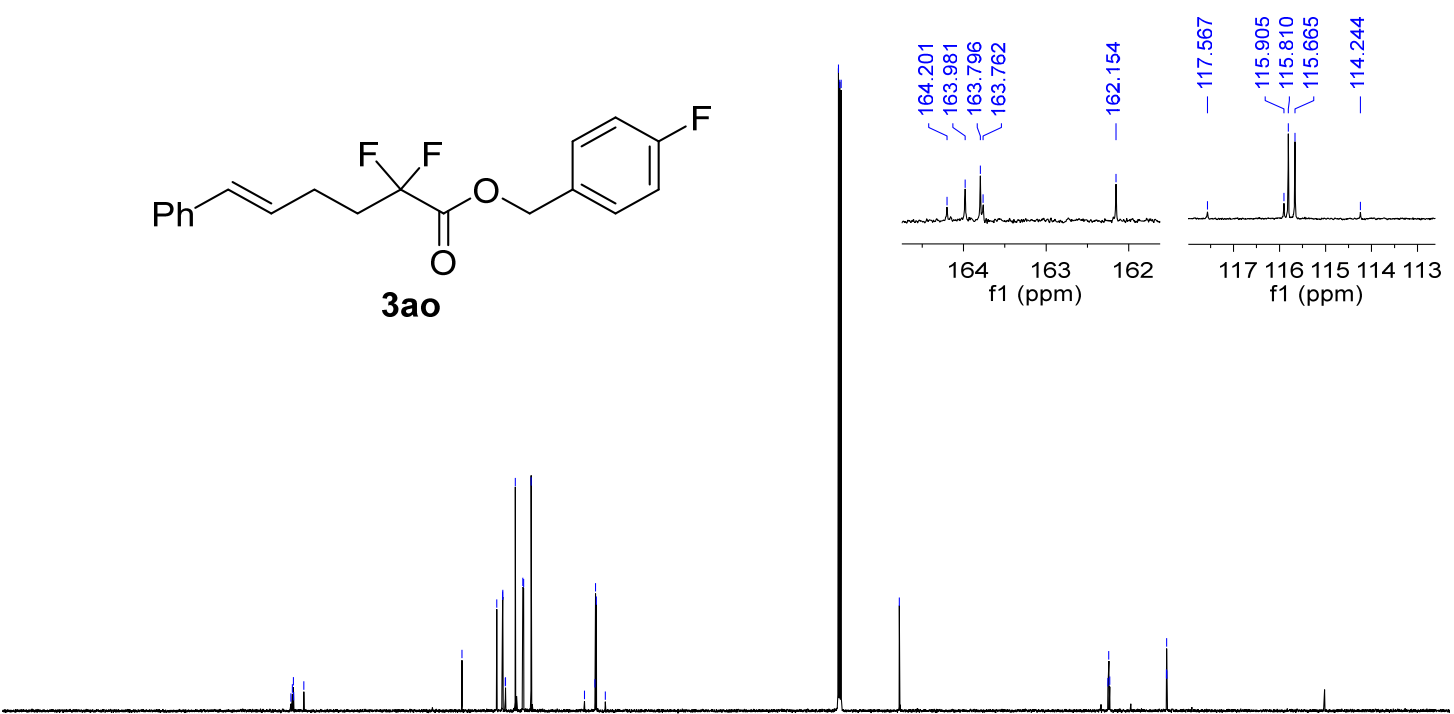

$\begin{array}{llllllllllllllllllllllll}10 & 200 & 190 & 180 & 170 & 160 & 150 & 140 & 130 & 120 & 110 & \begin{array}{l}100 \\ \mathrm{f} 1(\mathrm{ppm})\end{array} & 80 & 70 & 60 & 50 & 40 & 30 & 20 & 10 & 0 & -10 & -2\end{array}$

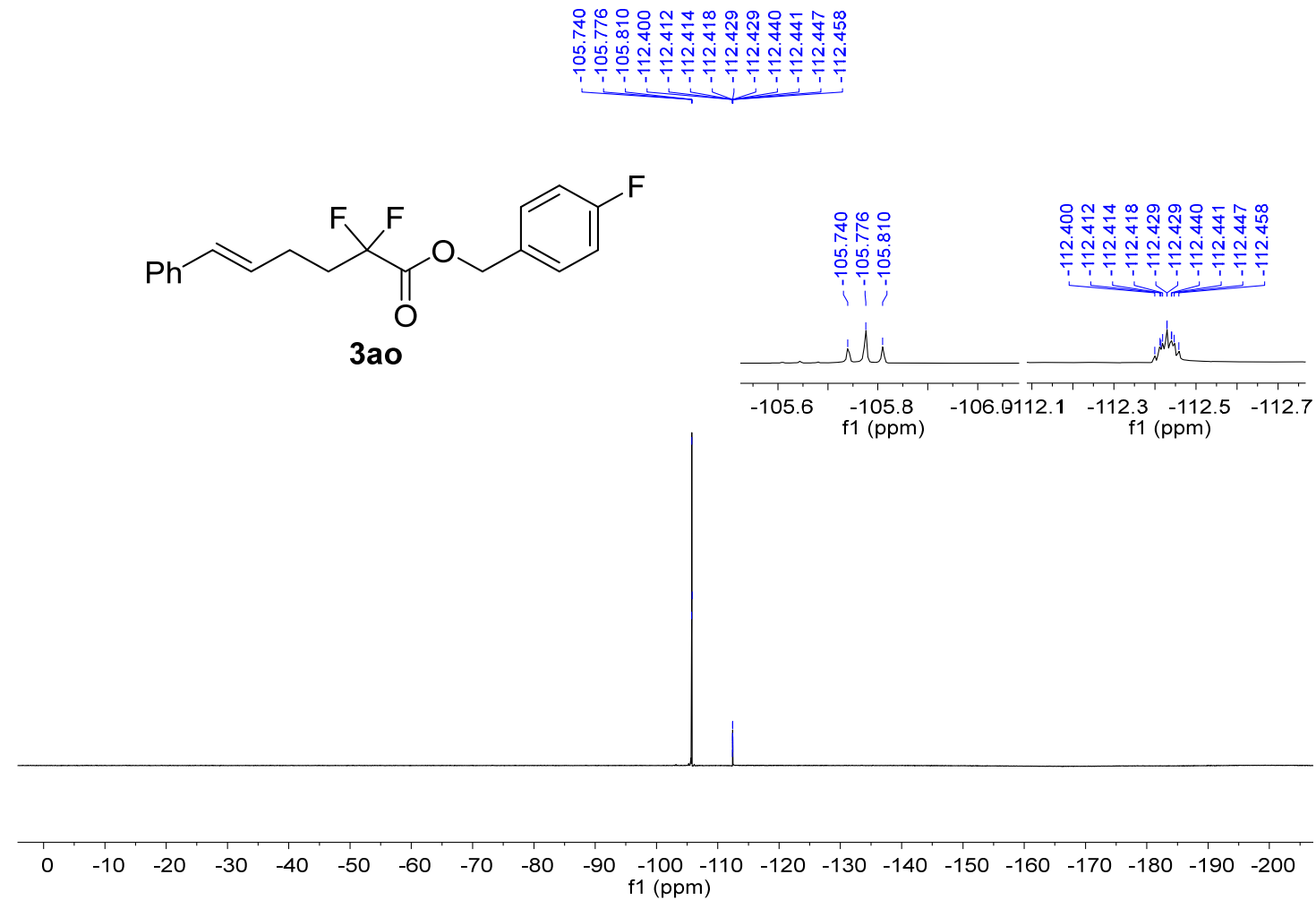


<smiles>O=C(N1CCOCC1)C(F)(F)CC/C=C/c1ccccc1</smiles>

3ap

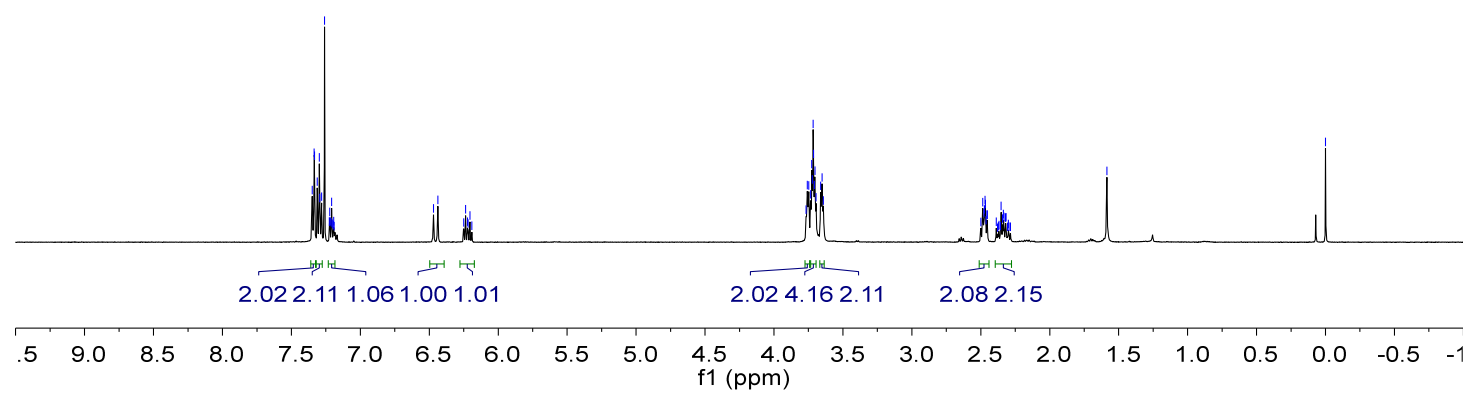

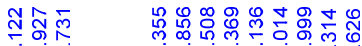

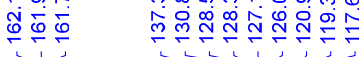

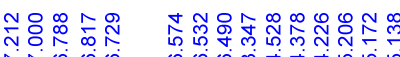

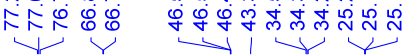<smiles>O=C(N1CCOCC1)C(F)(F)CC/C=C/c1ccccc1</smiles>

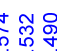

$\operatorname{lig}$

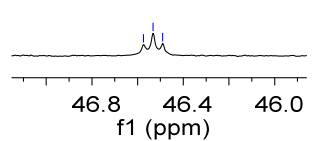

3ap

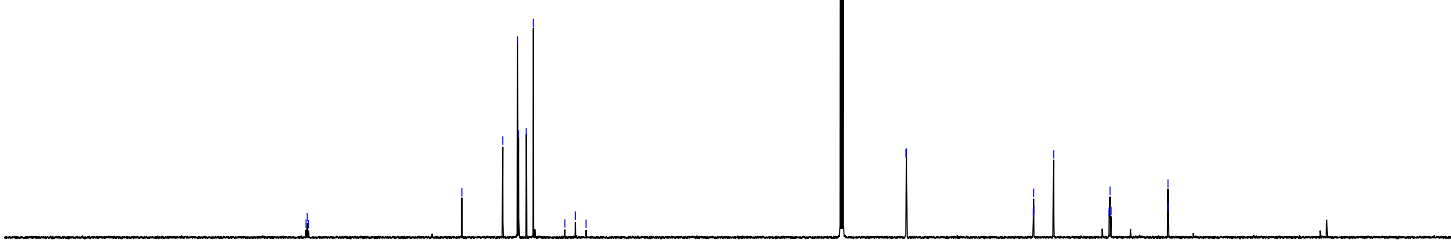

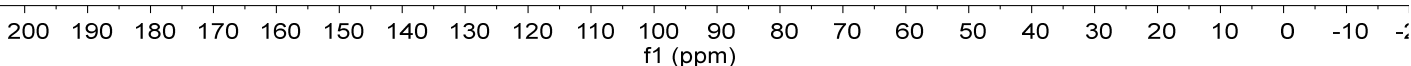


<smiles>O=C(N1CCOCC1)C(F)(F)CC/C=C/c1ccccc1</smiles>

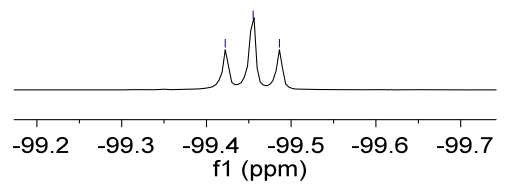

3ap

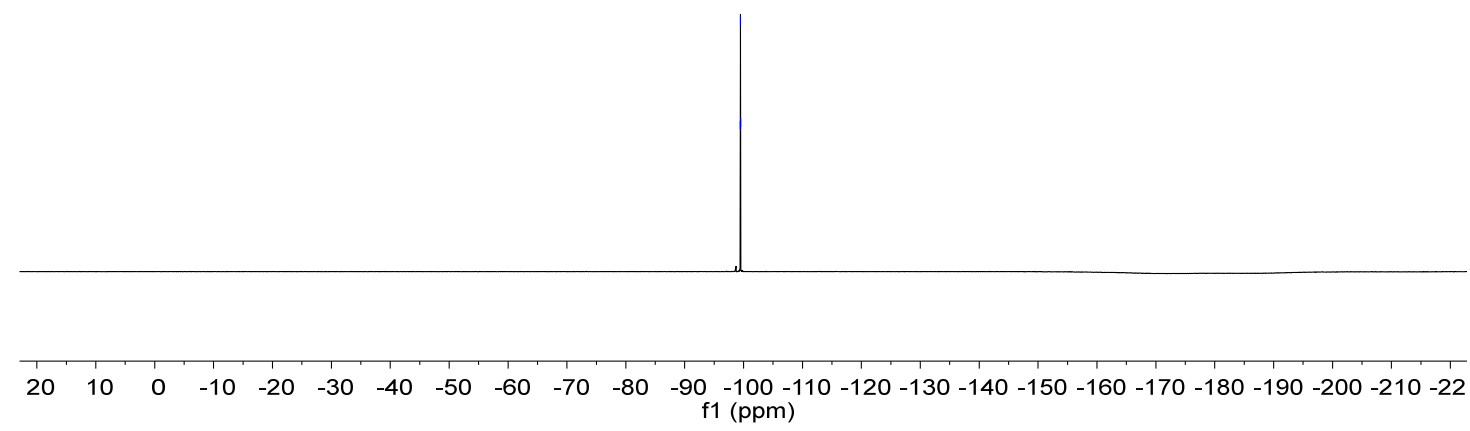<smiles>O=C(Nc1ccccc1)C(F)(F)CC/C=C/c1ccccc1</smiles>

$3 a q$

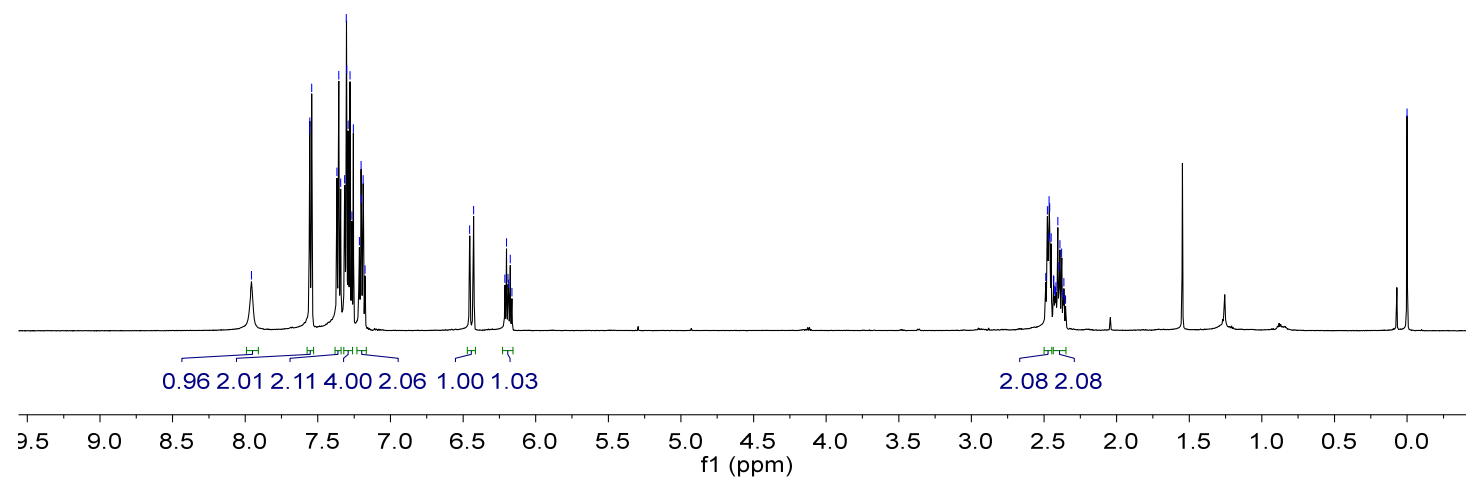


<smiles>O=C(Nc1ccccc1)C(F)(F)CC/C=C/c1ccccc1</smiles>

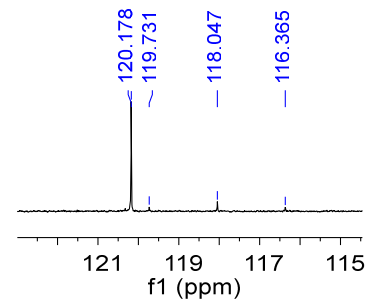

$3 a q$

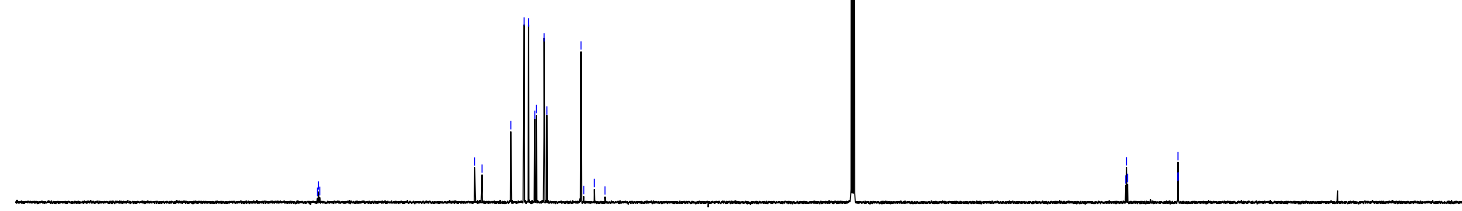

$\begin{array}{lllllllllllllllllllll}200 & 190 & 180 & 170 & 160 & 150 & 140 & 130 & 120 & 110 & \begin{array}{l}100 \\ \mathrm{f} 1(\mathrm{ppm})\end{array} & 90 & 70 & 60 & 50 & 40 & 30 & 20 & 10 & 0 & -10\end{array}$

정츄 윰

능ํํ응

(ppm)<smiles>O=C(Nc1ccccc1)C(F)(F)CC/C=C/c1ccccc1</smiles>

$3 a q$

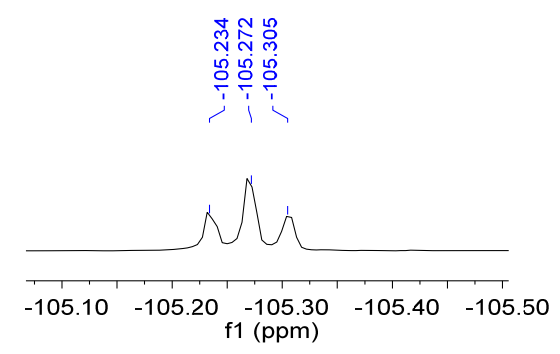

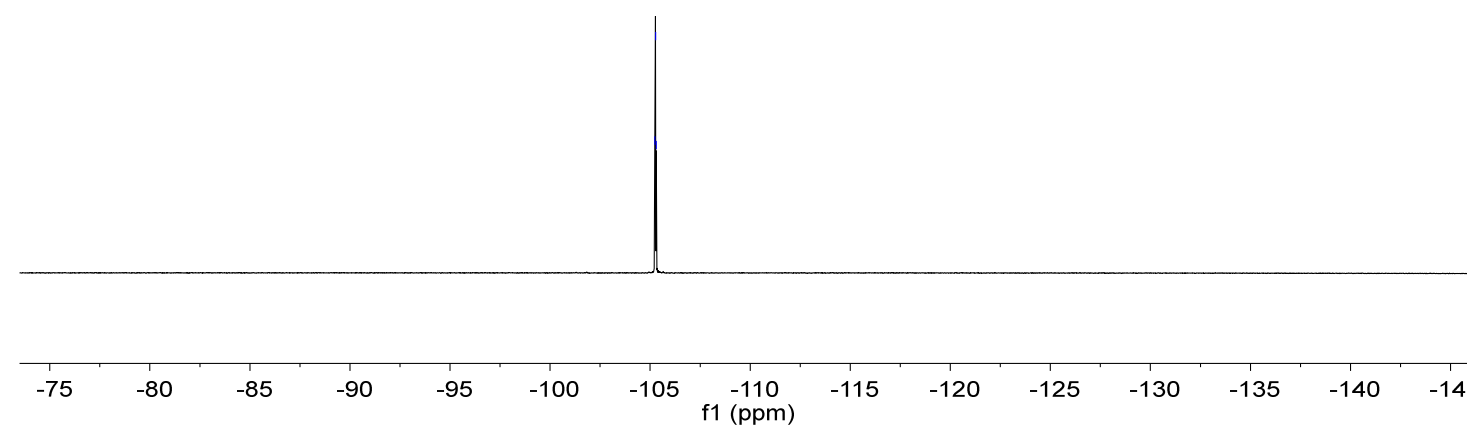



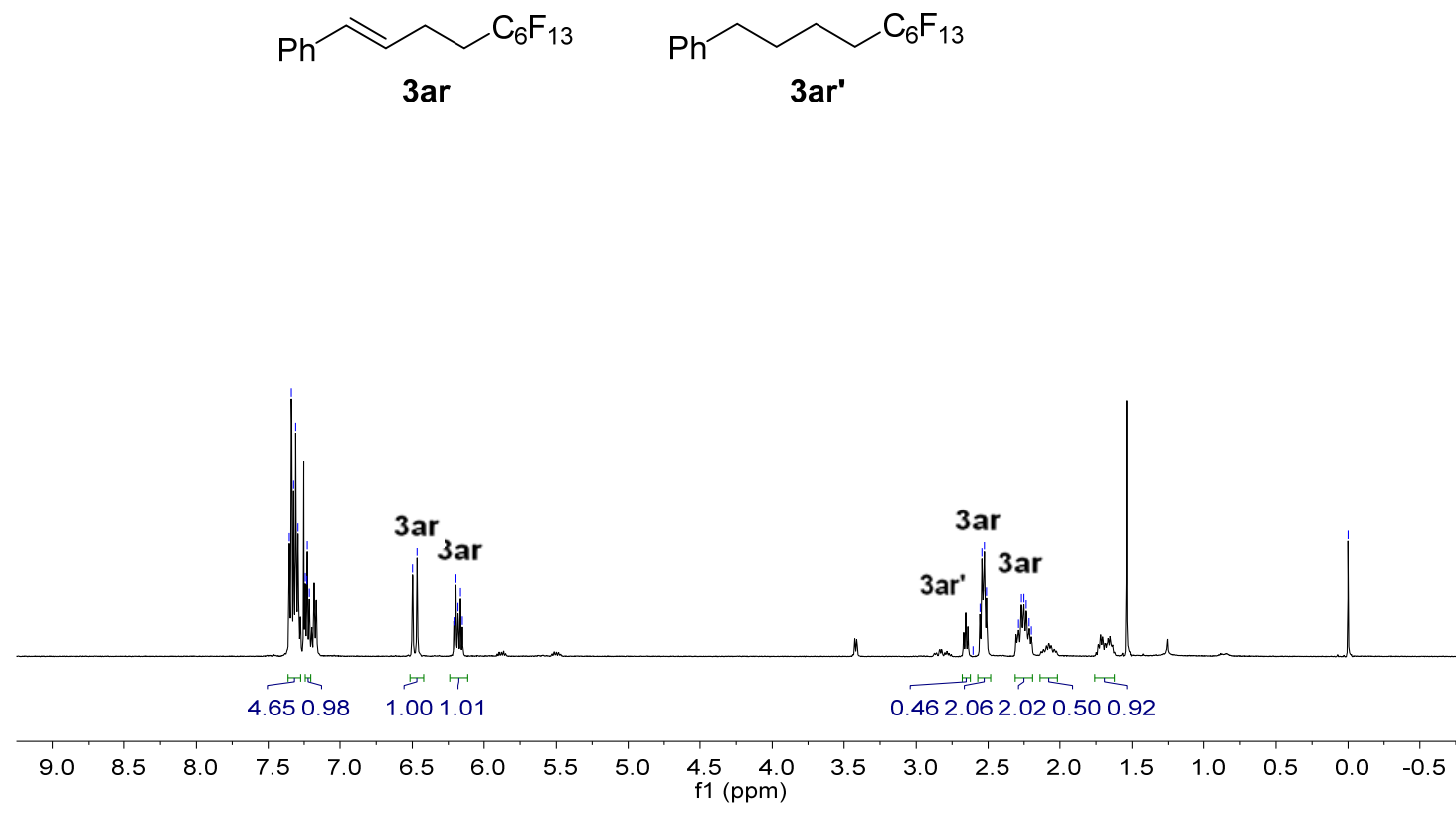

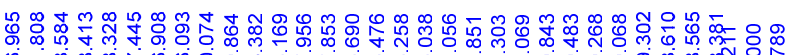

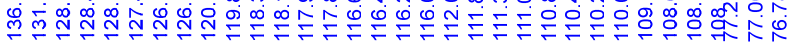

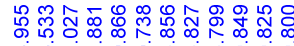

ळ

$\mathrm{Ph} \curvearrowright \mathrm{C}_{6} \mathrm{~F}_{13}$

3ar

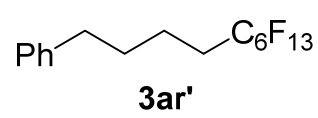

3ar'

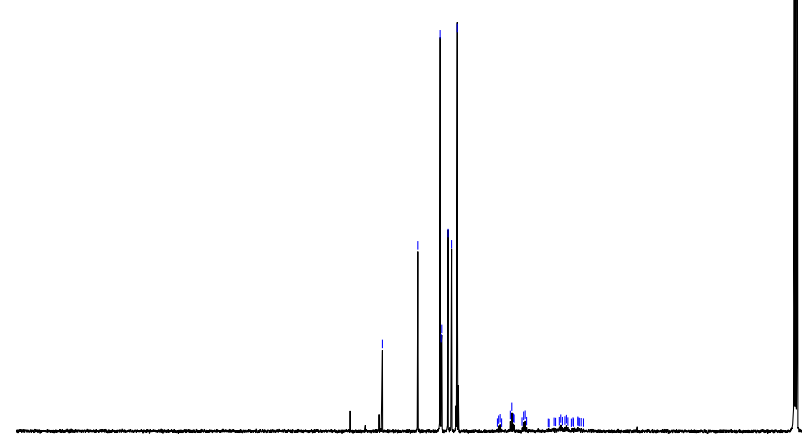

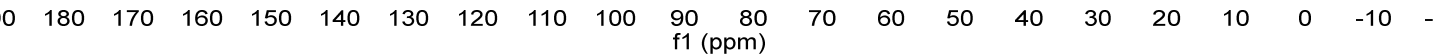


$\overbrace{3 a r} \mathrm{C}_{6} \mathrm{~F}_{13}$

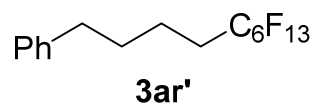

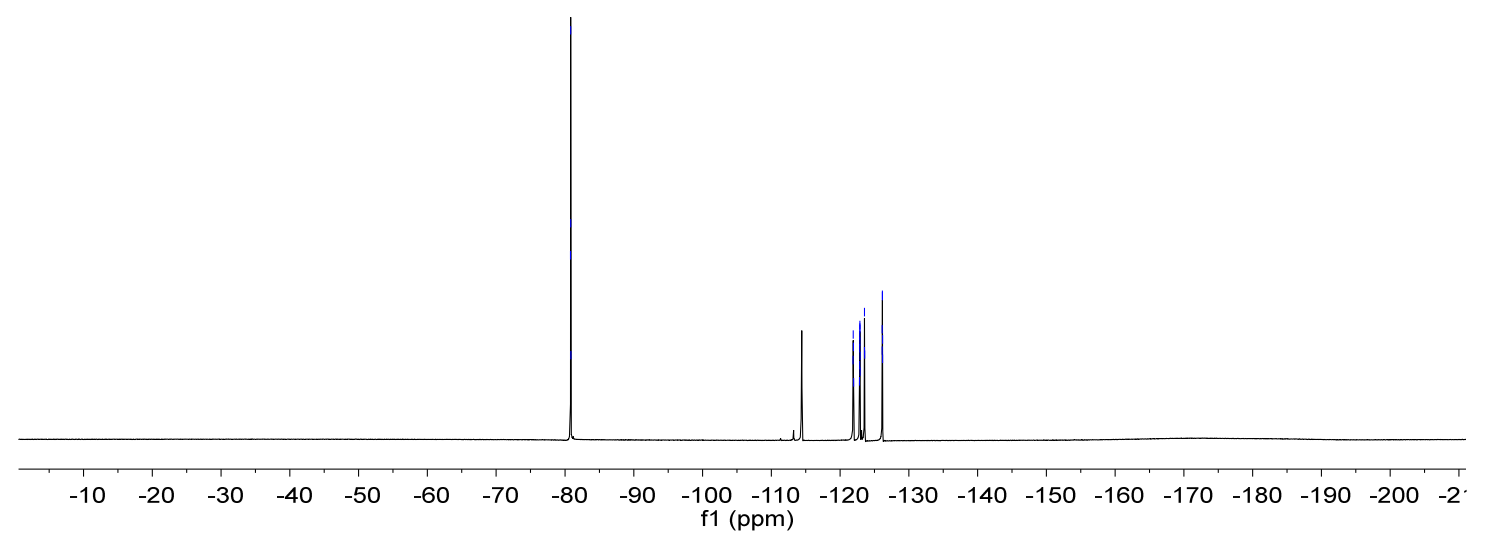

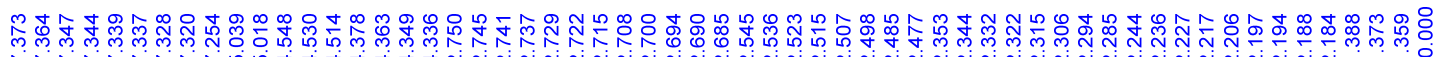

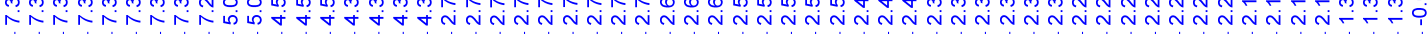<smiles>CCOC(=O)CCCC(Br)C(Br)c1ccccc1</smiles>

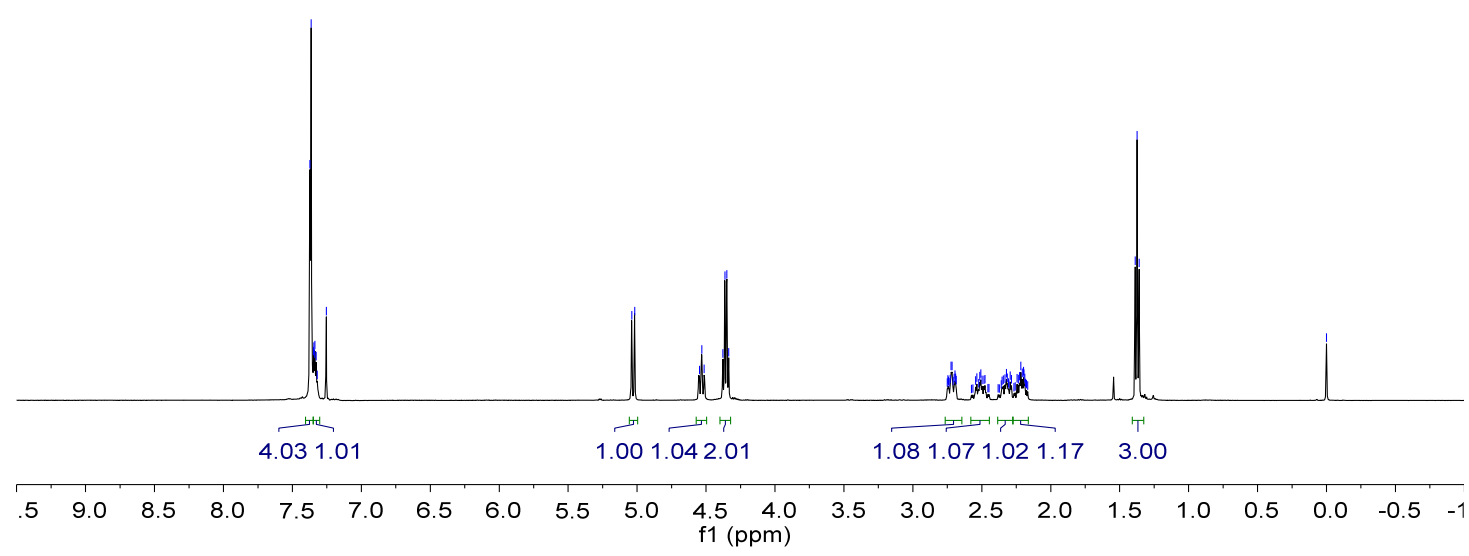




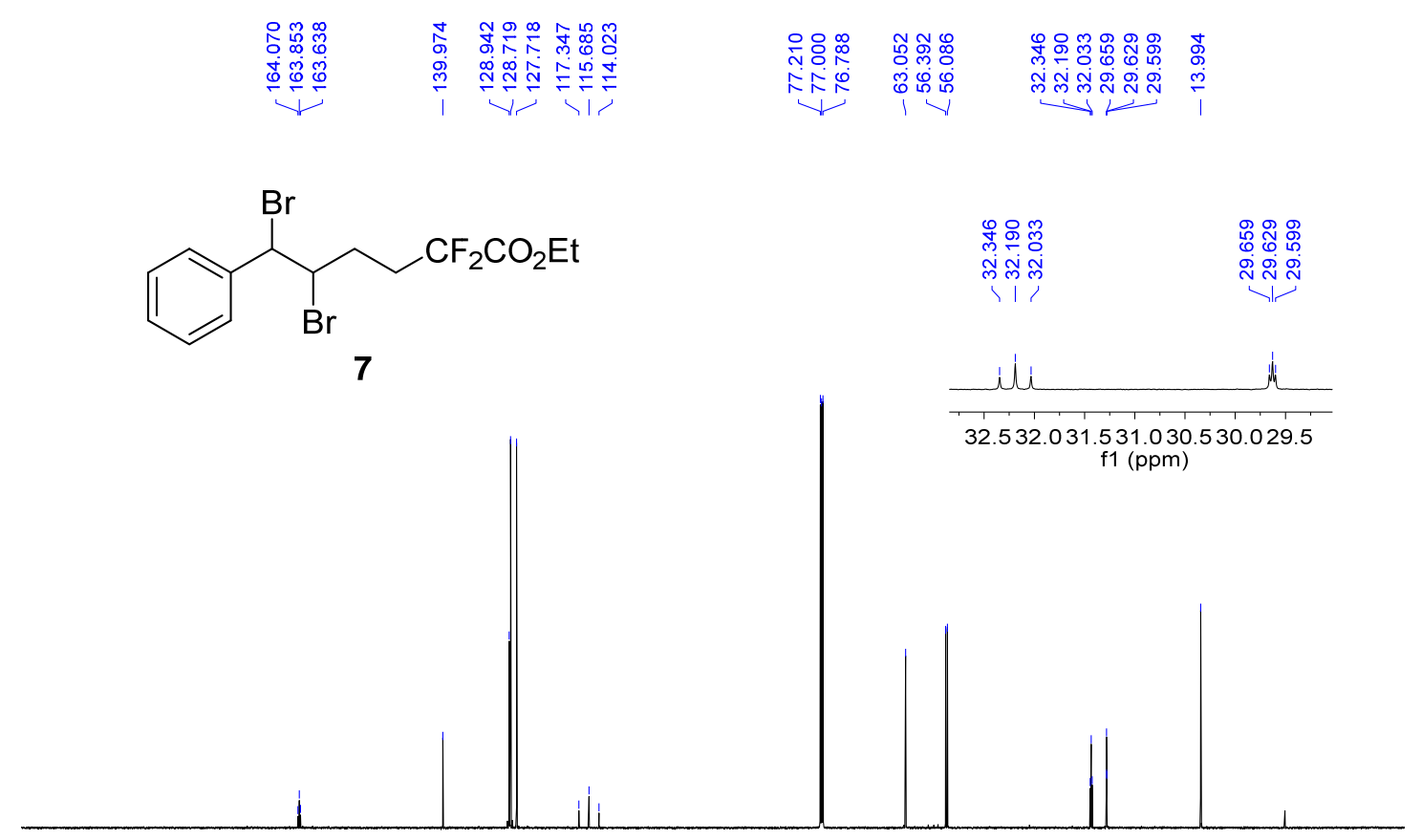

$\begin{array}{lllllllllllllllllllllll}10 & 200 & 190 & 180 & 170 & 160 & 150 & 140 & 130 & 120 & 110 & \begin{array}{l}100 \\ \mathrm{f} 1(\mathrm{ppm})\end{array} & 90 & 70 & 60 & 50 & 40 & 30 & 20 & 10 & 0 & -10 & -4\end{array}$

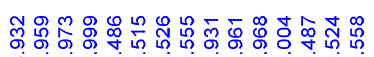

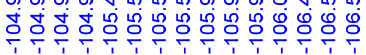

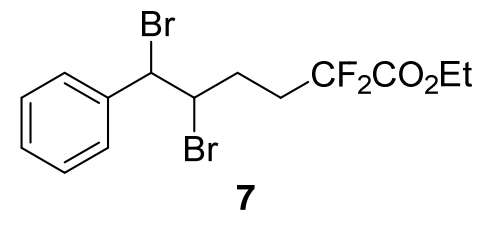

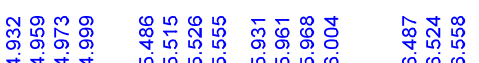

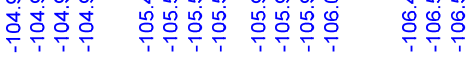

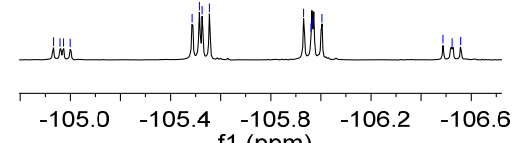

$\begin{array}{lllll}-105.0 & -105.4 & -105.8 & -106.2 & -106.6\end{array}$

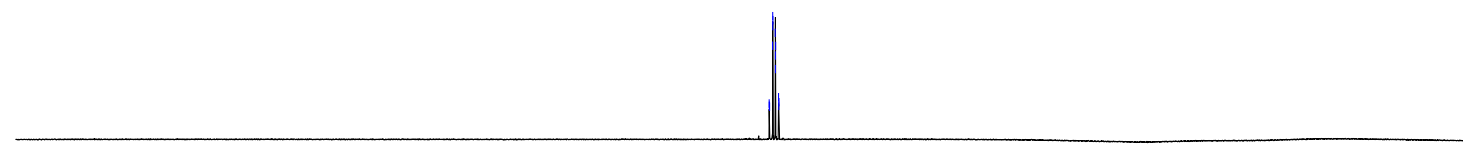

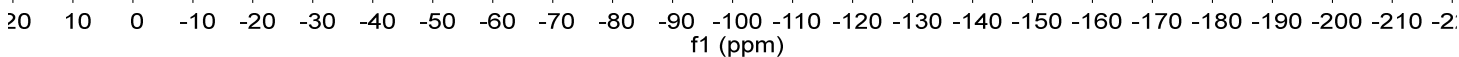



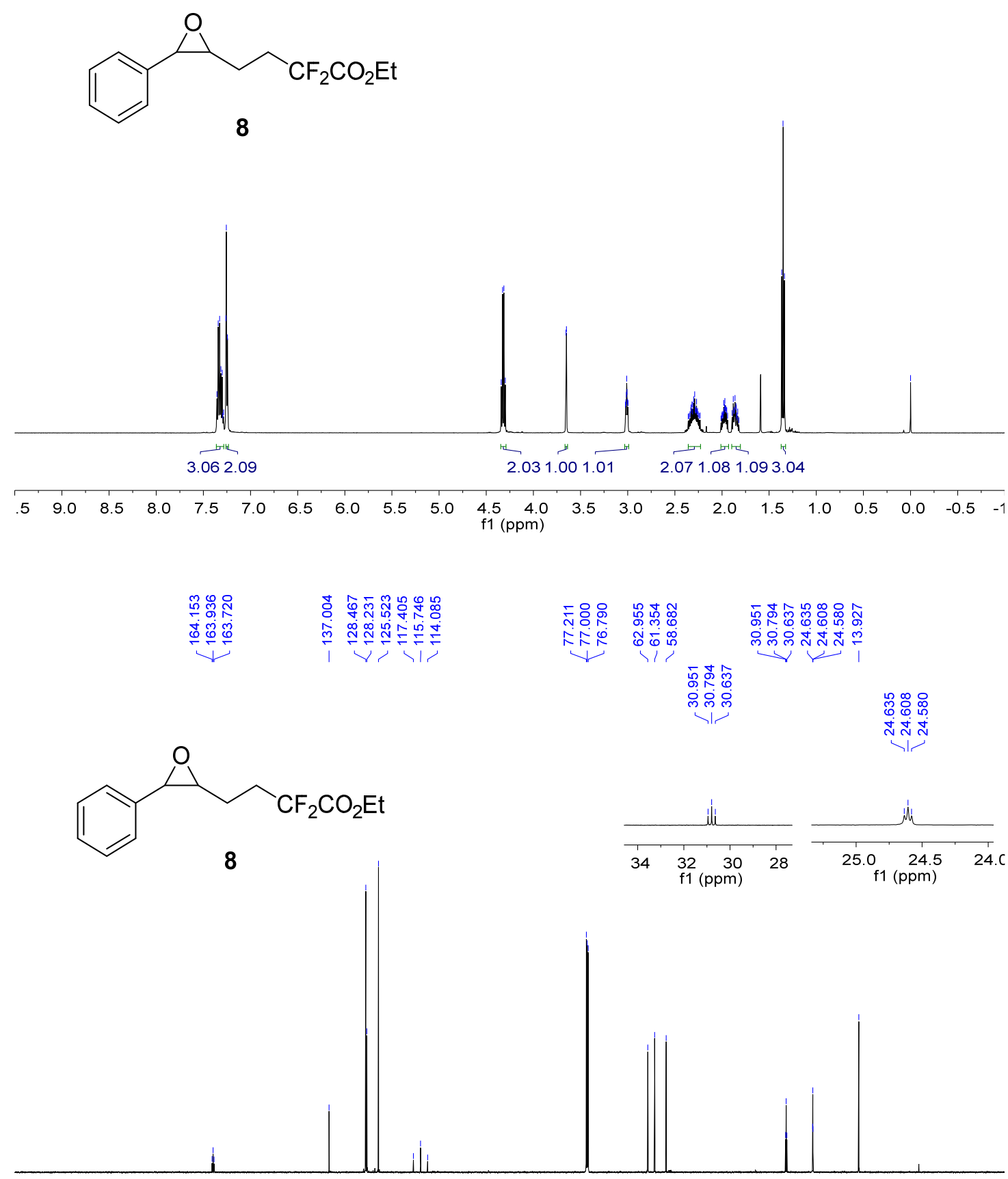

$\begin{array}{llllllllllllllllllllllll}10 & 200 & 190 & 180 & 170 & 160 & 150 & 140 & 130 & 120 & 110 & \begin{array}{l}100 \\ \mathrm{f} 1(\mathrm{ppm})\end{array} & 80 & 70 & 60 & 50 & 40 & 30 & 20 & 10 & 0 & -10 & -2\end{array}$ 


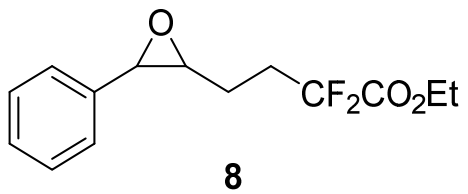

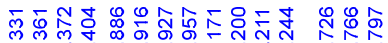

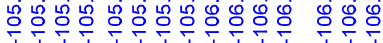

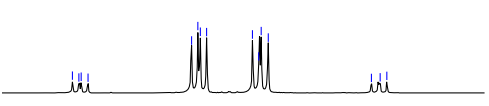

$-105.2-105.6-106.0-106.4-106.8-107.2$ f1 (ppm)

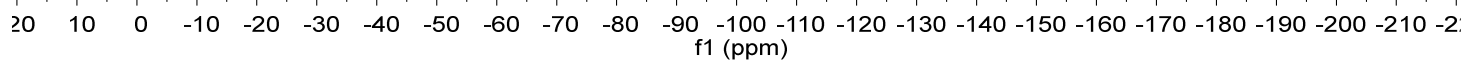

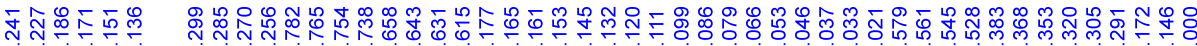

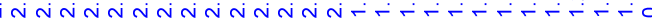

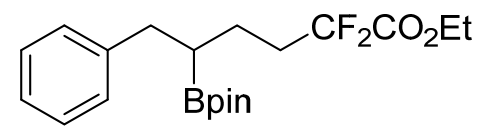

9

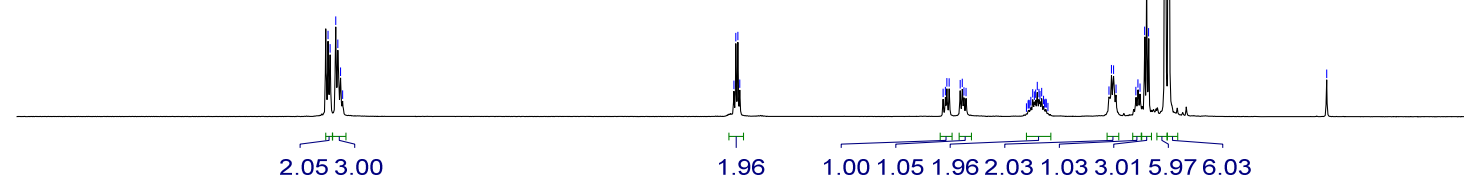

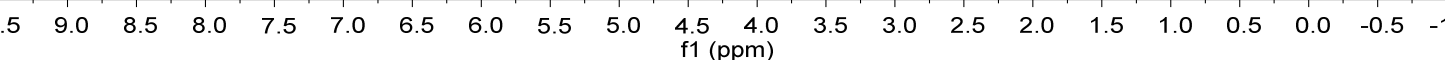




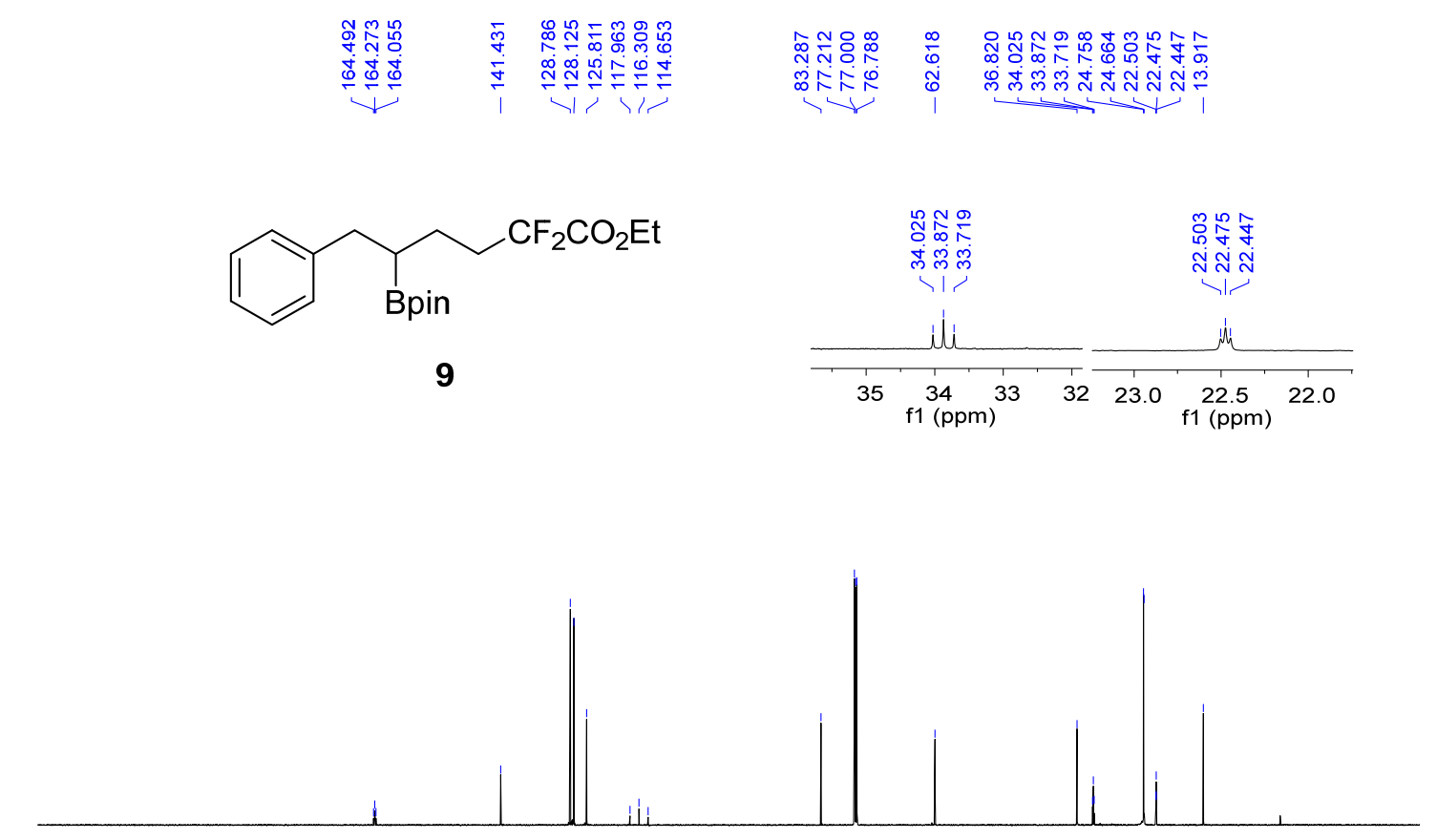

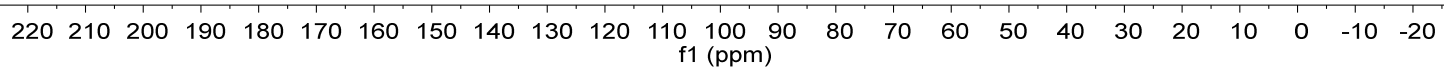

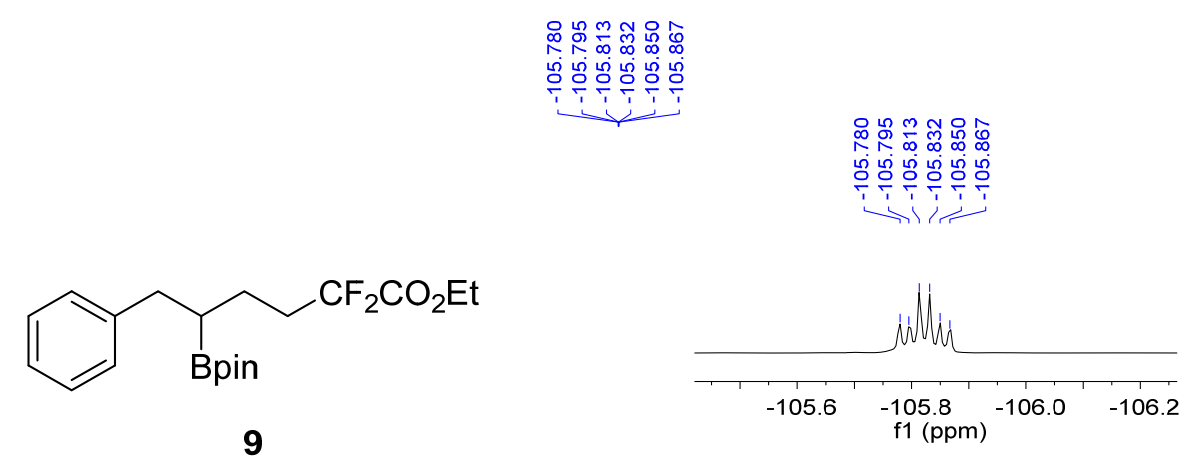

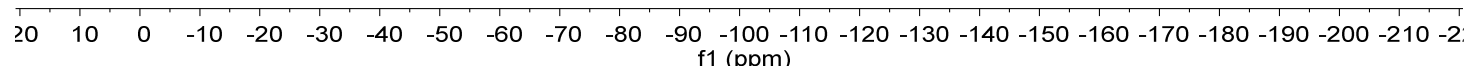




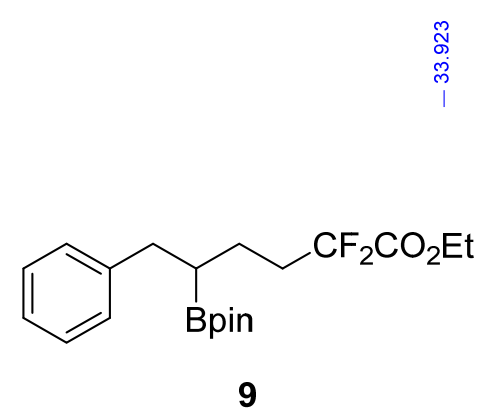

9

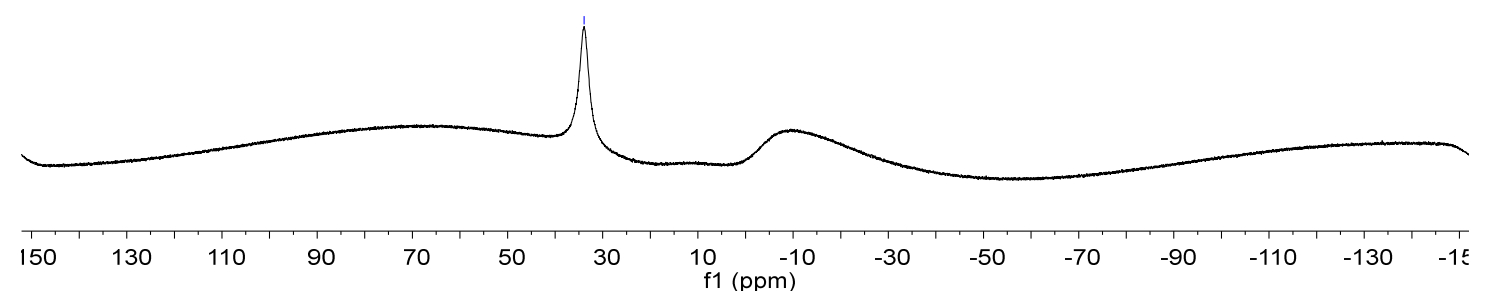

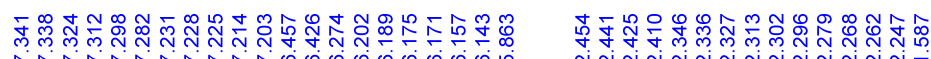

rinjarino<smiles>NC(=O)C(F)(F)CC/C=C/c1ccccc1</smiles>

10

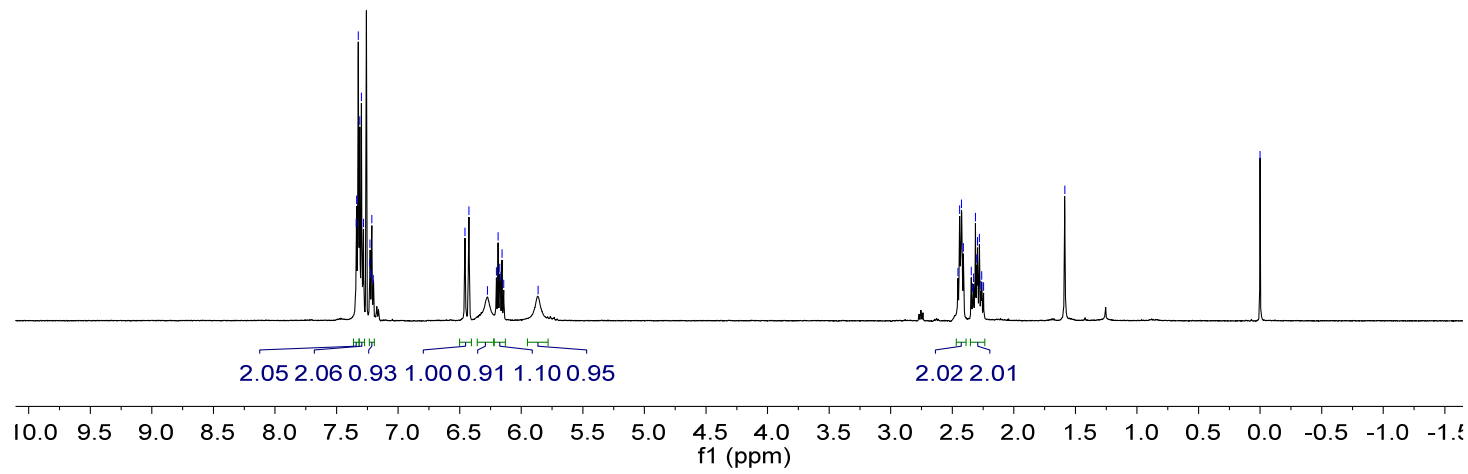




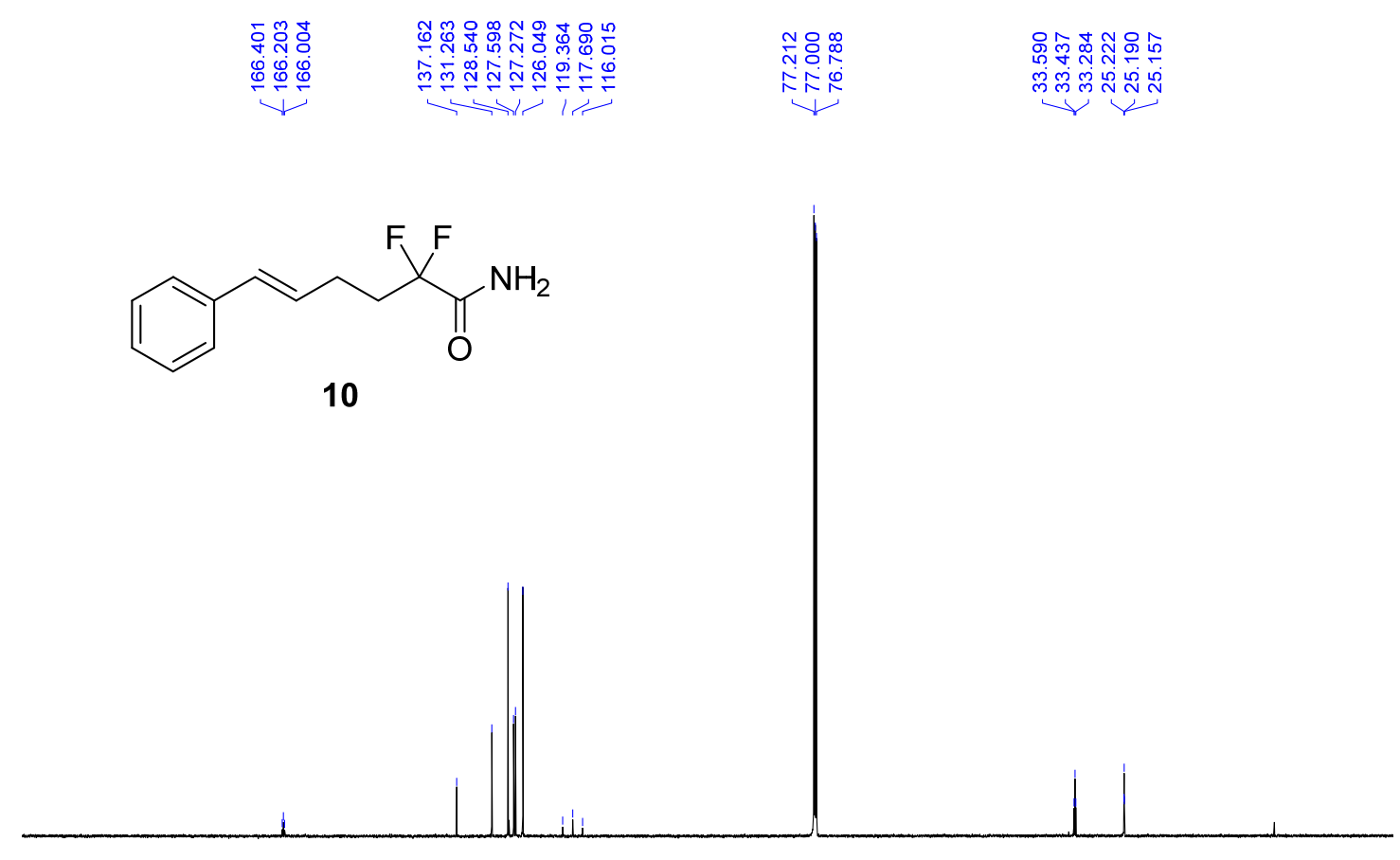

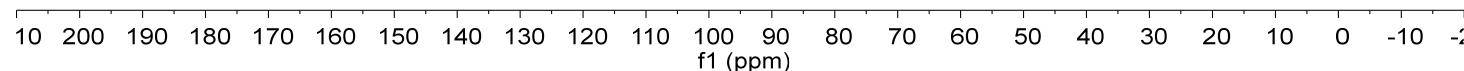

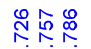

은<smiles>NC(=O)C(F)(F)CC/C=C/c1ccccc1</smiles>

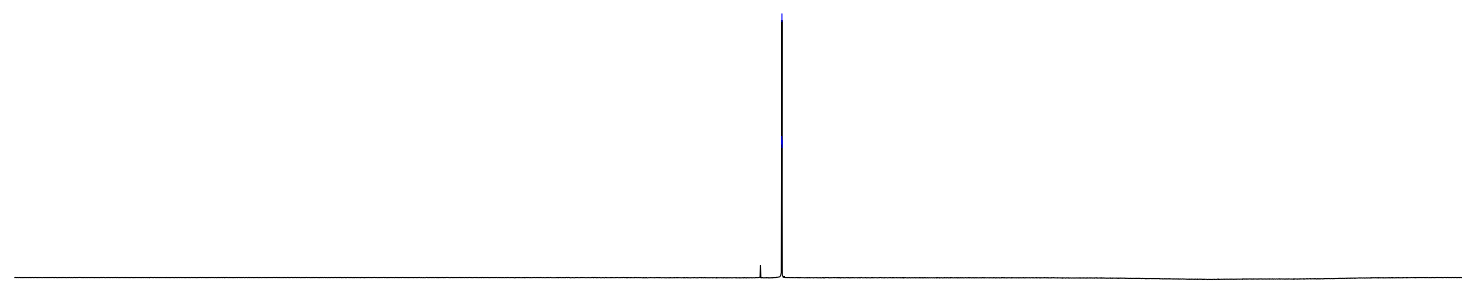

$\begin{array}{llllllllllllllllllllllllllll}10 & 0 & -10 & -20 & -30 & -40 & -50 & -60 & -70 & -80 & -90 & -100 & -110 & -120 & -130 & -140 & -150 & -160 & -170 & -180 & -190 & -200\end{array}$ 
<smiles>OCC(F)(F)CC/C=C/c1ccccc1</smiles>

11

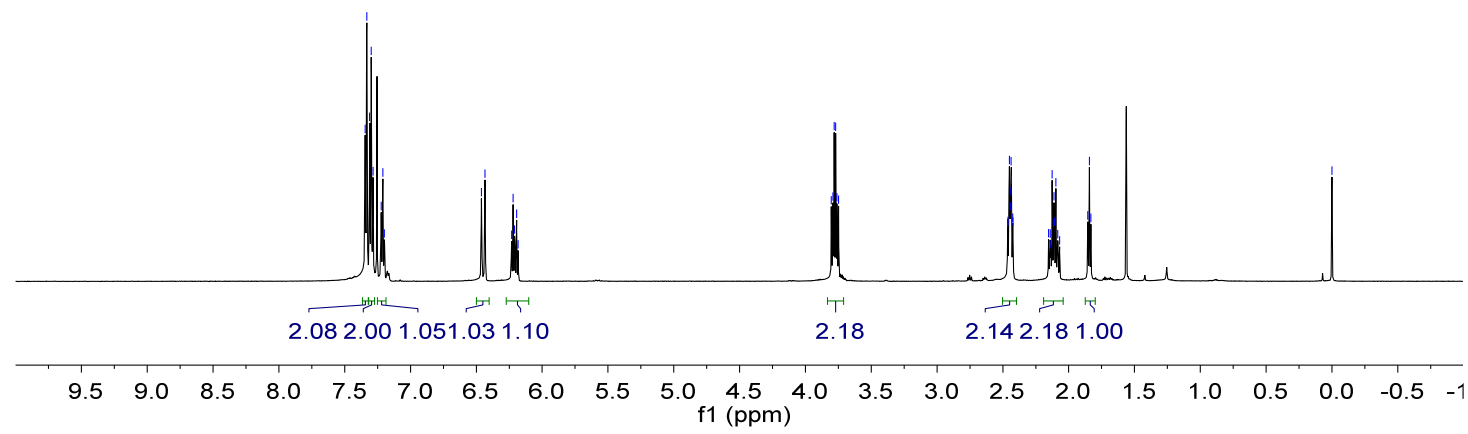

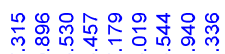

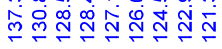

ஸ̣

Кㄴํ

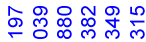

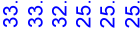

परत्य<smiles>OCC(F)(F)CC/C=C/c1ccccc1</smiles>

11

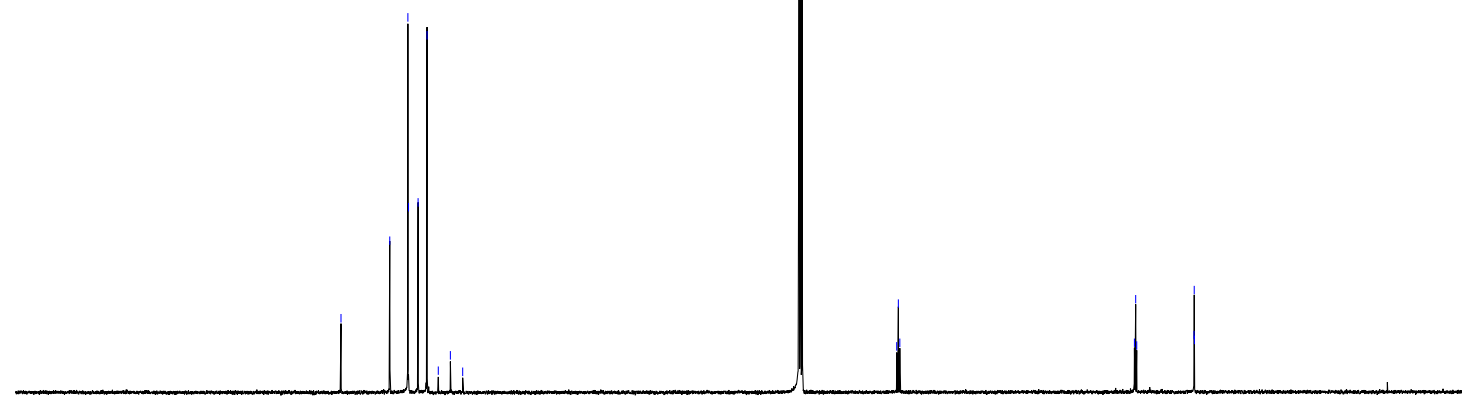

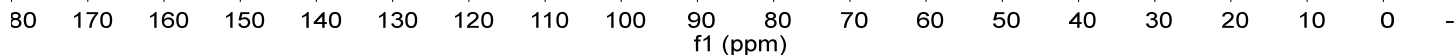



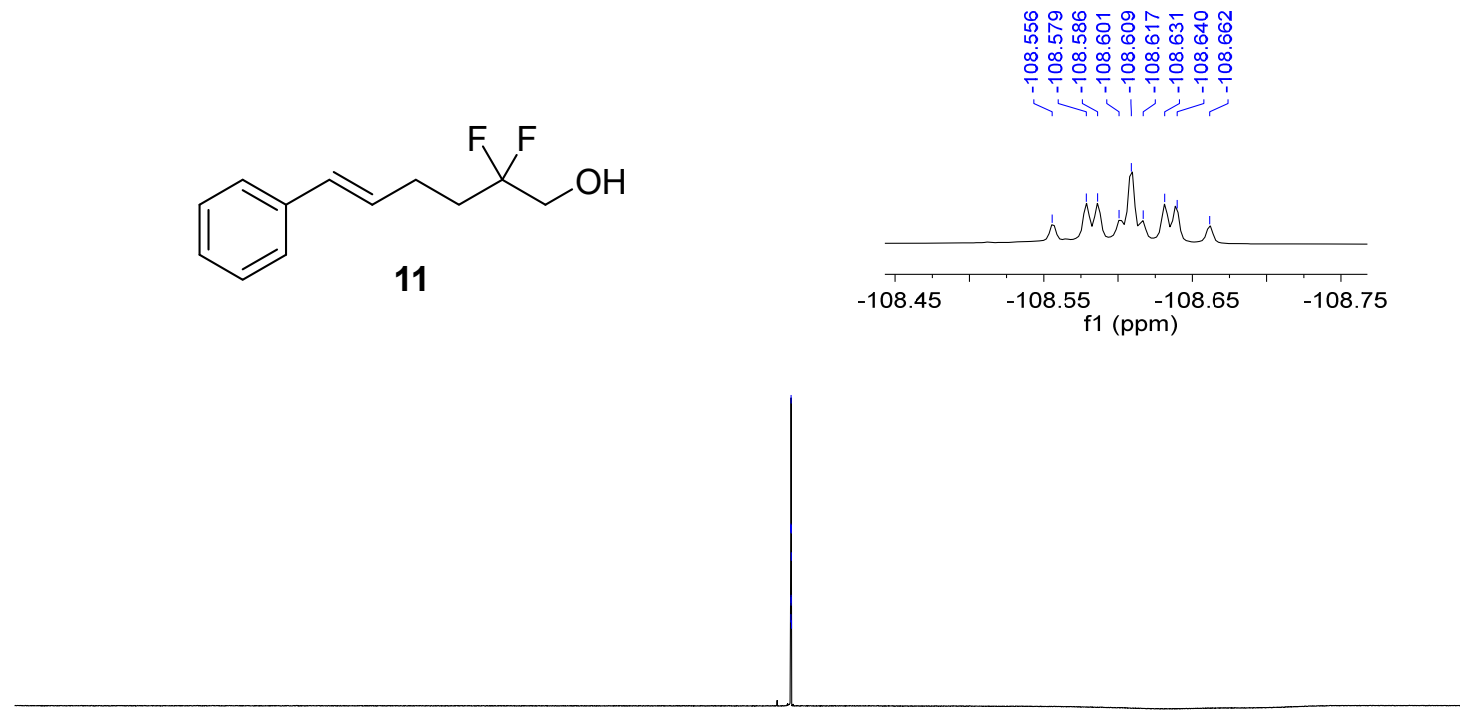

$\begin{array}{ccccccccccccccccccccccc}0 & 0 & -10 & -20 & -30 & -40 & -50 & -60 & -70 & -80 & -90 & -100 & -110 & -120 & -130 & -140 & -150 & -160 & -170 & -180 & -190 & -200 & -210\end{array}$

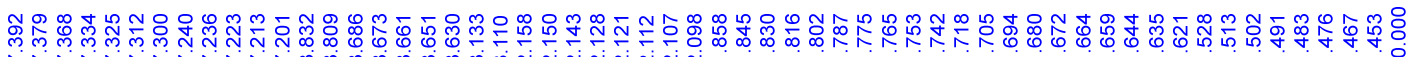

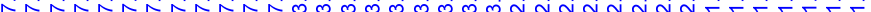<smiles>O=[Sn](c1ccccc1)C(CCCC(F)(F)CO)c1ccccc1</smiles>

13

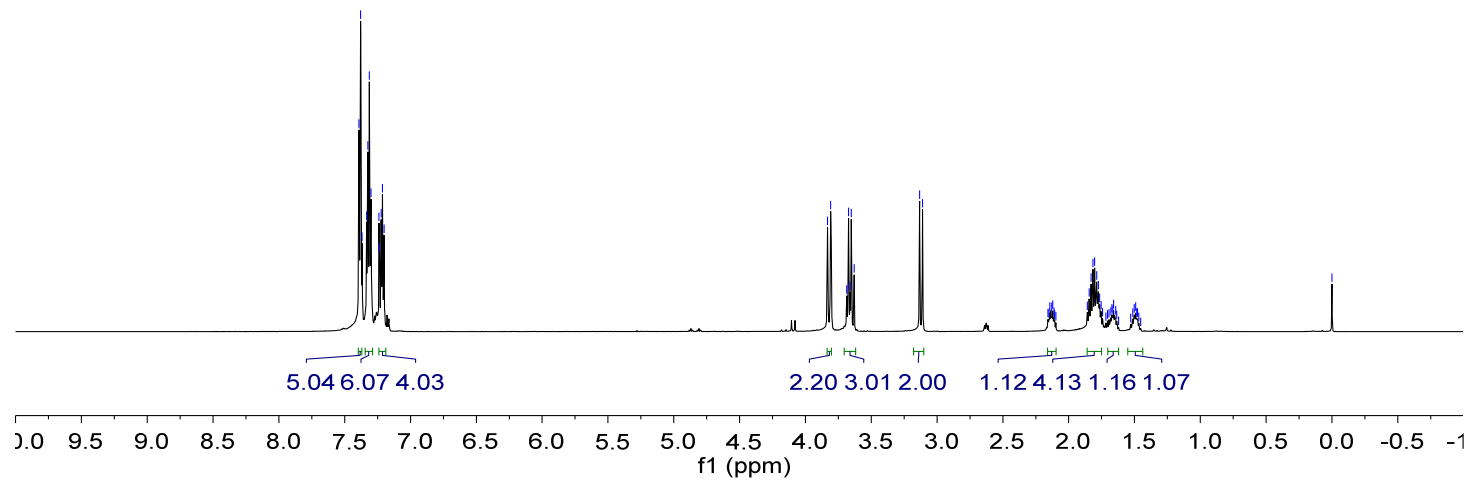


<smiles>OCC(F)(F)CCCC(Cc1ccccc1)c1ccccc1</smiles>

13
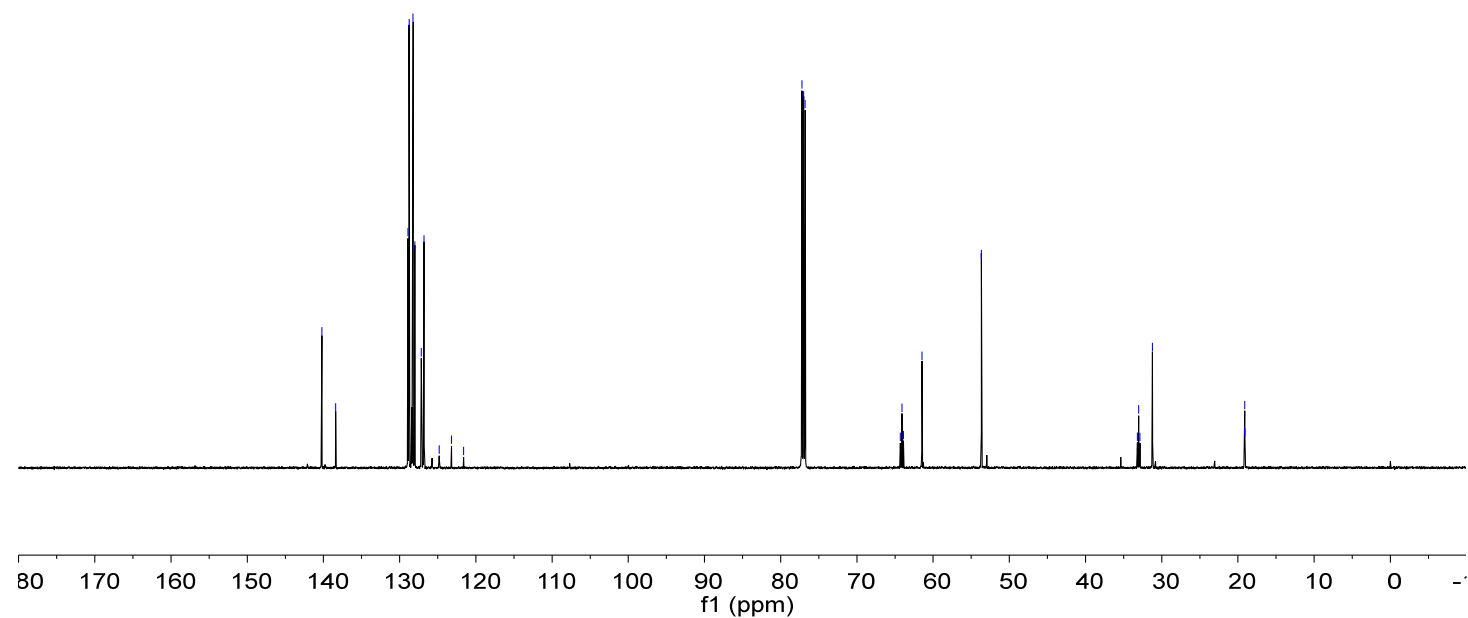

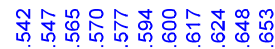

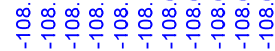

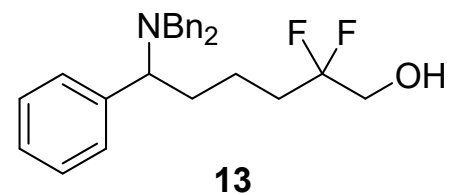

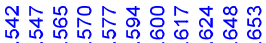

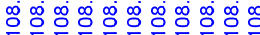

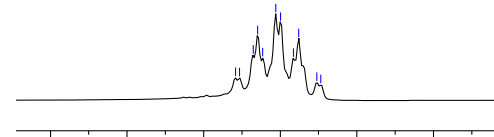

$-108.3-108.4-108.5-108.6-108.7-108.8$ f1 (ppm)

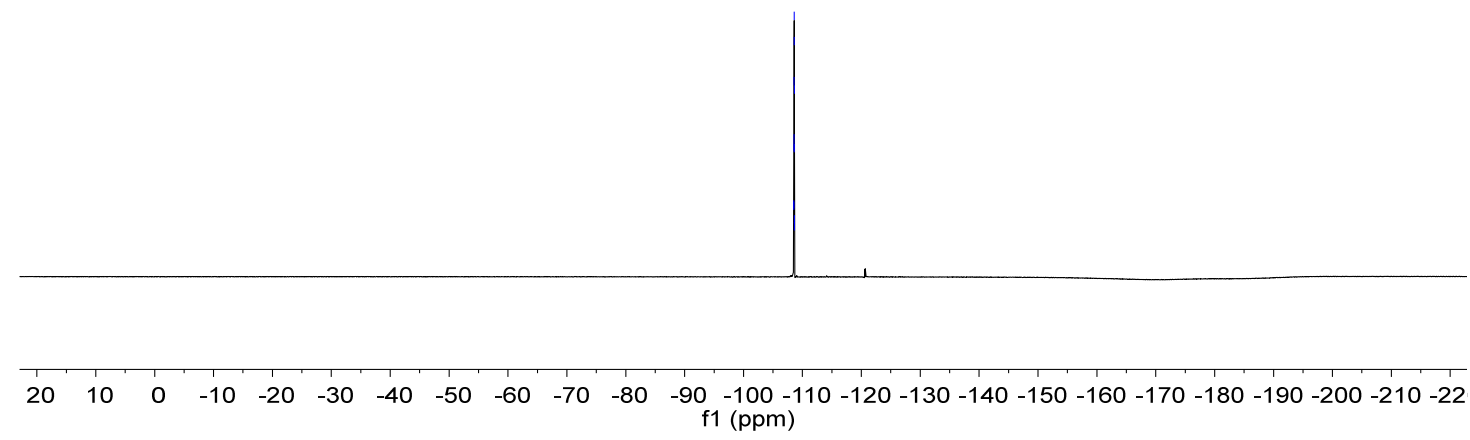

